\title{
Structural determinants of murine leukemia virus (MLV) reverse transcriptase (RT) important for fidelity and drug resistance in vivo
}

Elias Konstantine Halvas

West Virginia University

Follow this and additional works at: https://researchrepository.wvu.edu/etd

\section{Recommended Citation}

Halvas, Elias Konstantine, "Structural determinants of murine leukemia virus (MLV) reverse transcriptase (RT) important for fidelity and drug resistance in vivo" (2000). Graduate Theses, Dissertations, and Problem Reports. 1231.

https://researchrepository.wvu.edu/etd/1231

This Dissertation is protected by copyright and/or related rights. It has been brought to you by the The Research Repository @ WVU with permission from the rights-holder(s). You are free to use this Dissertation in any way that is permitted by the copyright and related rights legislation that applies to your use. For other uses you must obtain permission from the rights-holder(s) directly, unless additional rights are indicated by a Creative Commons license in the record and/ or on the work itself. This Dissertation has been accepted for inclusion in WVU Graduate Theses, Dissertations, and Problem Reports collection by an authorized administrator of The Research Repository @ WVU.

For more information, please contact researchrepository@mail.wvu.edu. 


\title{
Structural Determinants of Murine Leukemia Virus (MLV) Reverse Transcriptase (RT) Important for Fidelity and Drug-Resistance In Vivo
}

Elias Konstantine Halvas

Dissertation submitted to the School of Medicine and the Department of Biochemistry in partial fulfillment of the requirements for the degree of

Doctor of Philosophy

In

Biochemistry

\author{
Vinay K. Pathak, Ph.D., Chair \\ Daniel Flynn, Ph.D. \\ Charles L. Harris, Ph.D. \\ Wei-Shau Hu, Ph.D. \\ Michael Miller, Ph.D.
}

Department of Biochemistry

\section{Morgantown,West Virginia 2000}

Key Words: Murine Leukemia Virus (MLV), Reverse Transcriptase (RT), Fidelity, Drug-resitance

Copyright 2000 Elias Konstantine Halvas 


\section{ABSTRACT \\ Structural Determinants of Murine Leukemia Virus (MLV) Reverse Transcriptase (RT) Important for Fidelity and Drug-Resistance In Vivo}

\section{Elias Konstantine Halvas}

Error-prone DNA synthesis by retroviral reverse transcriptases (RTs) is a major contributor to variation in retroviral populations generating mutants that exhibit altered host tropisms, resistance to antiviral drugs, and the ability to escape the host's immune system. To identify structural elements of murine leukemia virus (MLV) RT important for fidelity and drug-resistance in vivo, we developed a D17-based encapsidating cell line (ANGIE P) which is designed to express the amphotropic MLV envelope. ANGIE P cell line also contains an MLV-based retroviral vector (GA-1), which encodes a wild-type bacterial $\beta$-galactosidase gene (lacZ) and a neomycin phosphotransferase gene. Transfection of ANGIE P cells with wild type or mutated MLV gag-pol expression constructs generated GA-1 virus that was able to undergo only one cycle of viral replication upon infection of D17 cells. The infected D17 cell clones were characterized by staining with $\mathrm{X}-\mathrm{Gal}$ and the frequencies of inactivating mutations in lac $Z$ were quantified. Several structural determinants of MLV RT were identified that were important for fidelity which included position V223 of the YVDD box, several residues of the dNTP-binding site, as well as residues residing in the RNase $\mathrm{H}$ domain of MLV RT. A number of these MLV RT mutants resulted in statistically significant decreases in fidelity (1.2 to 2.8-fold) whereas two mutants showed a statistically significant increase in fidelity (0.8-fold) relative to wild-type MLV RT. Furthermore, these amino acid residues were observed to play critical roles in catalysis and viral replication, which was exhibited by the reductions in both viral titers as well as RT activities of these mutants. In addition to identifying structural determinants important for fidelity, we also showed that the V223 position of MLV RT may not be the only structural determinant important for resistance to the antiviral nucleoside analog, 2', 3'-dideoxy-3'-thiacytidine (3TC). This is in contrast to results observed with human immunodeficiency virus type 1 (HIV1) RT. These results establish a sensitive in vivo assay for identification of structural determinants important for accuracy of DNA synthesis as well as dug-resistance and indicate that several structural determinants may have an effect on the in vivo fidelity of MLV RT. 


\section{ACKNOWLEDGMENTS}

This work is dedicated to my family, especially to my parents Mr. Konstantine and Mrs. Angeliki Halvas, to my brother George Halvas as well as to my dear friend Mary Gobbie.

To my parents that encouraged me to be my very best, I thank them from the bottom of my heart. I owe all of my achievements, now and forever to them. This is but a mere gesture of my gratitude towards them and I could never begin to repay them for all they have done for me. To my brother George, thanks for your support and above all, for your crazy perspective and philosophy on life. You've definitely made my life considerably more interesting and enjoyable.

To my dear friend Mary Beth Gobbie, if my family has provided me with encouragement, you have bestowed upon my life a sense of direction. Thank you Mary for your support and for giving my life a bit of long needed focus. Furthermore, I would like to acknowledge that I am aware of the sacrifices you have endured on behalf of my training and education. Thank you for being a part of my life for the last ten years and hopefully for the years to come.

In terms of my education and career, I will like to thank Vinay Pathak, my mentor, advisor, and friend for granting me the opportunity to work in his lab. It has been a wonderful five and a half years. Thank you Vinay for providing me, through your guidance, the knowledge and the valuable skills a young scientist as myself will require and utilize throughout my career. It has been an honor and a privilege to work with you.

I would also like to thank Wei-Shau Hu who has been like a second mentor to me. Thank you Wei-Shau also for being a committee member as well as a friend, and I appreciate the guidance and intellectual input you have provided for my projects during my graduate career.

I would also like to extend my gratitude to my other committee members, Dan Flynn, Larry Harris, and Mike Miller for your distinct insights and contributions, which each of you has provided to my projects. I would also like to thank Dan Flynn for making me work it at my last committee meeting and to Larry Harris for making me rewrite the discussion of my thesis. I'm a little bit more humble with a substantially better final chapter. And to Mike Miller, thank you for your crazy stories about hunting deer and the awesome pictures of road kill.

I would like to thank all the remaining faculty, students, and all other personnel of the Biochemistry Program for helping me, even if it were in the most trivial manner. You were all a great help in one way or another during my graduate career. Thank you all for all that you have done.

To Dr. Christine Milcarek and Dr. Nirmala SunderRaj, I would like to thank both of you for the wonderful and pleasant experiences I encountered in each of your labs and for 
stimulating my interest in science early on. It was both a pleasure and an honor working for both of you and do not change a thing about yourselves.

To my older cousins, Mary, Mike, and George Spanos, Vickie and Anna Pedos, and Spiro Petrogannis, thank you for being the older brothers and sisters a never had. Through your experiences I have learned lessons in life that will stay with me forever. To my younger cousins, Anna and Kelly Pedos, Kosta, Anna, and Toula Halvas, and Elias and Georgia Petrogannis, thank you for your wit, humor and vitality which comes with youth. You have all made me realize how far I have come, but to never forget what I have passed. And to my Goddaughter Georgie, thank you for making me realize what is really important in life.

To my friends, Ben Beasley, Nick Contis, Bill Copetas (good and bad), Ted Kim, Bob Malley, and Leo Pandelidis, thanks for all the beer, debauchery, and good times. My experiences with you guys have helped smooth out all the rough edges in life. And to "Mauvro", the one who provided me with alternative titles to my thesis such as, "The never ending story". Well George, at least this story has ended and the title is somewhat different than you would expect.

I would like to thank all the members of Dr. Pathak's and Dr. Hu's lab both past and present for everything thing they have done. Each one of you has helped me in one way or another and your help has not gone unnoticed. I would especially like to thank Jenny Svarovskaia and Ben Beasley for their valuable input and for intellectual discussions pertaining to my projects.

"Life is one great adventure, full of good and bad; take it all in, and never be sad" 


\section{TABLE OF CONTENTS}

Title page,

pg. i

Abstract,

pg. ii

Acknowledgments, pgs. iii-iv

Table of Contents, pgs. v-vi

List of Tables and Illustrations, pgs. vii-X

\section{Chapter 1:}

Introduction and Review of Literature,

pgs. $1-16$

Figures,

pgs. $17-28$

References,

pgs. $29-45$

\section{Chapter 2:}

Development of an In Vivo Assay to Identify structural Determinants in Murine Leukemia Virus Reverse Transcriptase Important for

Fidelity, pgs. $46-63$

Tables and Figures, pgs. $64-72$

References, pgs. $73-83$

\section{Chapter 3:}

Structural determinants in the putative dNTP binding site of

MLV RT that are important for in vivo fidelity during reverse transcription, pgs. $84-105$

Tables and Figures, pgs. 106-118

References, pgs. 119-126

\section{Chapter 4:}


Wildtype and YMDD Mutant of Murine Leukemia Virus Reverse

Transcriptase are Resistant to 2', 3'-dideoxy-3'-thiacytidine

(3TC),

pgs. $127-138$

Tables and Figures,

pgs. $139-144$

References,

pgs. $145-154$

\section{Chapter 5:}

Discussion,

pgs. $155-178$

Tables and Figures,

pgs. $179-186$

References,

pgs. $187-202$

\section{Appendix A}

Figures,

pgs. $203-222$

\section{Abstracts}

Cold Spring Harbor Abstracts,

pgs. 223-225

\section{Curriculum Vitae}

CV,

pgs. $226-230$ 


\section{LIST OF TABLES AND ILLUSTRATIONS}

\section{Chapter 1: Review of Literature}

Figure 1. Retroviral genomic structure,

pgs. $17-18$

Figure 2. Retroviral replication,

pgs. $19-20$

Figure 3. Reverse transcription,

pgs. $21-22$

Figure 4. Crystal structure of HIV-1 RT complexed with nevirapine (Kohlstaedt et al, 1992),

pg. 23

Figure 5A. Crystal structure of HIV-1 RT complexed with template-primer (Jacobo-Molina et al, 1994),

pgs. $24-25$

Figure 5B. Crystal structure of HIV-1 RT complexed with templateprimer and incoming dTTP substrate (Huang et al, 1998), pg. 26

Figure 6. Crystal structure of a catalytically active fragment of MLV RT (Georgiadis et al, 1995),

pgs. $27-28$

\section{Chapter 2: Development of an In Vivo Assay to Identify Structural Determinants in}

\section{Murine Leukemia Virus Reverse Transcriptase Important for Fidelity}

Table 1. Effects of V223 mutations in the YXDD box of

MLV RT on the frequency of lacZ inactivation,

pgs. 64-65

Table 2. Effects of mutations in the RNase $\mathrm{H}$ domain of

MLV RT on the frequency of lacZ inactivation, pg. 66

Figure 1. Structures of MLV-based constructs and rapid in vivo assay to identify structural determinants in MLV RT important for fidelity,

pgs. $67-68$ 
Figure 2. Comparison of RT activities and viral titers,

pgs. 69-70

Figure 3. PCR analysis of lacZ mutations in proviral DNAs of infected cell clones,

pgs. 71-72

Chapter 3: Structural determinants in the putative dNTP binding site of MLV RT that are important for in vivo fidelity during reverse transcription

Table 1. Effects of mutations in the putative dNTP binding site of MLV RT on the frequency of $l a c Z$ inactivation,

pgs. 106-108

Table 2. Effects of F156 mutations in MLV RT on the frequency of lacZ inactivation,

pgs. $109-110$

Table 3. Effects of mutations in residues in proximity to residues of the putative dNTP binding site of MLV RT on the frequency of lac $Z$ inactivation,

pgs. 111-112

Table 4. Reverse transcriptase activities of mutants associated with the putative dNTP binding site of MLV RT, pgs. $113-114$

Figure 1. Structures of MLV-based constructs and rapid in vivo assay to identify structural determinants in MLV RT important for fidelity, pgs. $115-116$

Figure 2. dNTP binding sites of MLV and HIV-1 RTs,

pgs. $117-118$

\section{Chapter 4: Wildtype and YMDD Mutant of Murine Leukemia Virus Reverse Transcriptase are Resistant to $2^{\prime}, 3^{\prime}$-dideoxy-3'-thiacytidine (3TC)}

Table 1. The effect of 3TC treatment on MLV replication, pgs. $139-140$ 
Figure 1. Structures of MLV-based constructs and protocol utilized to determine sensitivity to 3TC in NIH 3T3 and 143B cell lines,

pgs. $141-142$

Figure2. HIV-based vector expressing luciferase reporter and activation of 3TC in NIH3T3, Hela, 143B, and D17 cell lines,

pgs. $143-144$

\section{Chapter 5: Discussion: Fidelity, Drug-Resistance, and the Evolution of Retroviruses}

Table 1. Distance comparison of side chains between YXDD box and dNTP-binding site residues of HIV-1 and MLV RTs, pg. 179

Table 2. Distance comparison of $\mathrm{C} \alpha-\mathrm{C} \alpha$ bonds between YXDD box and dNTP-binding site residues of HIV-1 and MLV RTs, pg. 180

Table 3. Distance comparison of side chain distances between residues constituting the dNTP-binding site of HIV-1 or MLV RTs, pg. 181

Table 4. Distance comparison of the $\mathrm{C} \alpha-\mathrm{C} \alpha$ bonds between residues constituting the dNTP-binding sites of HIV-1 or MLV RTs, pg. 182

Figure 1. Comparison of the HIV-1 and MLV RT active sites, pgs. $183-184$

Figure 2. Comparison of RT activities and viral titers of dNTPbinding site mutants, pgs. $185-186$ 


\section{Appendix A: Plasmid Construction}

Figure 1. Construction of V223A, M, and S mutants of MLV RT, pgs. 204-205

Figure 2. Construction of the V223I mutant of MLV RT,

pgs. 206-207

Figure 3. Construction of the Q190E mutant of MLV RT,

pgs. $208-209$

Figure 4. Construction of the Q190H and N mutants of MLV RT, pgs. 210-211

Figure 5. Construction of RMBNB derivative of pLGPS,

pgs. $212-213$

Figure 6. Construction of dNTP binding site mutants of MLV RT

utilizing double-stranded DNA oligonucleotides

(Scheme I),

pgs. 214-215

Figure 7. Construction of dNTP binding site mutants of MLV RT utilizing double-stranded DNA oligonucleotides (Scheme II),

pgs. 216-217

Figure 8. Construction of dNTP binding site mutants of MLV RT utilizing double-stranded DNA oligonucleotides (Scheme III),

Figure 9. Construction of dNTP-binding site mutants of MLV RT by PCR-based mutagenesis,

pgs. $220-222$ 


\section{CHAPTER 1}

Introduction and Review of Literature 
Retroviruses are pseudo-diploid, single-stranded (ss) RNA viruses which replicate through a double-stranded (ds) DNA intermediate. This ds DNA eventually becomes integrated into the genome of their host where it is known as the provirus. Thus, these viruses require a life cycle that utilizes a unique viral polymerase (reverse transcriptase) as well as cellular DNA and RNA polymerases $(17,99)$. These viruses are members of the Retroviridae family that exhibits some of the most genetic diversity present in nature, giving an inherent genetic heterogenecity known as the quasispecies (28).

This genetic heterogenecity arises from an intrinsically high mutation rate and a large population size $(22,28)$. The high error rate is due in large part to the nature of reverse transcription (RT) during viral replication and recombination. Heterogenecity can also arise as a result of the host polymerases' fidelity, which are also utilized in the retroviral life cycle. Regardless of the mechanism, mutations arise that increase the variability of the population and in turn can increase the fitness of the viral population.

This phenomena of increased fitness is evident in populations of human immunodeficiency virus type 1 (HIV-1) where mutations arise that can confer drug resistance to both nucleoside and nonnucleoside analogs $(21,29,96)$. In addition, escape mutants that evade the immune system can also be selected for through changes in viral antigen epitopes $(69,79)$, thus hindering efforts to develop effective vaccines (103). Thus, the collapse of a host's immunological response and a failure in the efficacy of antiviral drugs may accelerate the progression of acquired immunodeficiency syndrome (AIDS), which results from HIV-1 infections. Therefore, dissection of the mechanisms 
by which this variation arises may provide us with important insights on how viruses like HIV-1 evolve.

Genomic structure of retroviruses. Infections resulting from members of the Retroviridae family have been identified in a number of vertebrate hosts including, mammals, fish, birds, reptiles and even insects $(17,55,81)$. The genomes of these viruses contain two copies of plus-strand RNA ranging between 7-10 kb in length which are modified in the same manner as eukaryotic mRNAs. These modifications include, $5^{\prime}$ capping and $3^{\prime}$ polyadenylation as well as methylation $(12,17,51)$. The genomes of these viruses are arranged into three genes with the nomenclature gag, pol and, env (Fig. 1). The gag gene, encoding several structural proteins forming the core of the virus, is transcribed and translated as a polyprotein that is cleaved into (MA), capsid (CA) and nucleocapsid (NC) $(17,46,78)$. The pol gene encodes RT and integrase (IN), two polypeptides residing in the viral particle core and employed in the early steps of retroviral replication $(1,17)$. In addition, a region of the genome between gag and pol codes for an aspartic protease (PR) required during the processing of the polypeptide precursors into their respective subdomains $(17,68)$. Depending on the retroviral species, PR can be expressed as a part of gag as observed in avian leukemia virus (ALV) or as a part of pol, as seen in murine leukemia virus (MLV) (17). Finally, the env gene codes for a glycosylated polyprotein translated from spliced subgenomic RNA and processed by cellular proteases. The resultant products of the processed polypeptide are designated as the surface (SU) and the transmembrane (TM) subunits of the viral surface glycoprotein that protrudes from the enveloped surface of the virus. This protein 
complex is utilized during the attachment and entry of the virus into the target cell (17, 48).

The retroviral RNA genome also possesses non-coding sequences functioning as cis-acting elements with vital roles during reverse transcription, integration, transcription, splicing and packaging (Fig. 1). At the $5^{\prime}$ and $3^{\prime}$ ends of the viral RNA are identical sequences termed $\mathrm{R}$ (repeated sequences) important during the obligatory jumps of reverse transcription (17). Sequences directly down stream of the $5^{\prime} \mathrm{R}$ include the unique $5^{\prime}$ sequence (U5), which comprise of elements critical for termination of transcription (Poly A) and attachment during integration (att). In addition to U5, the primer binding site (PBS), the site where reverse transcription initiates, is also present directly down stream of $5^{\prime} \mathrm{R}(16,17,37)$. Additional cis-acting sequences at the $5^{\prime}$ end of the retroviral genome include the splice donor site utilized in the generation of subgenomic mRNA followed by the packaging signal designated as either $\Psi$ or $E$ (17). The cis-acting elements of the unique $3^{\prime}$ region (U3) of the retroviral genome include the polypurine tract (PPT), the second att site and sequences possessing transcriptional enhancer activity $(17,37,59)$.

Retroviral replication. A complex set of events transpires in the movement of genetic information from RNA to DNA and back to RNA. This is characterized as the retroviral life cycle (Fig. 2), which begins with virion attachment to the cell through protein-protein interactions between viral surface glycoprotein and cellular receptor (17). Attachment of the viral particle to the cell surface receptor promotes fusion of both envelopes leading to internalization of the viral particle and release of the nucleocapsid complex into the cytoplasm. Reverse transcription proceeds with conversion of the 
single-stranded RNA genome into double-stranded DNA and eventually, the integration of this DNA into the host's genome by integrase. This provirus in turn is transcribed, processed, and translated by host machinery including RNA polymerase II, spliceosomes, and ribosomes respectively. These translated proteins are targeted to the cellular membrane, assemble into viral particles packaging two full-length transcripts of the viral genome and finally bud from the cell. During budding, processing of the polypeptides by viral protease occurs which leads to the generation of mature virions. This newly released particle is prepared for another round of viral infection (17).

Reverse transcription. An important event in the retroviral life cycle is the process known as reverse transcription converting single-stranded RNA into doublestranded DNA (Fig. 3) (17, 37). This transformation takes place in two distinct stages and two sets of templates are employed. In the first stage, reverse transcription initiates at the PBS using a virus-specific cellular tRNA as a primer and proceeds to the $5^{\prime}$ end of the RNA molecule copying the U5 and $5^{\prime} \mathrm{R}$. This short DNA product known as minusstrand strong stop DNA, is transferred to the $3^{\prime}$ end of the genome, during the first obligatory jump. This jump is thought to be facilitated by the RNase $\mathrm{H}$ domain of RT that degrades the RNA component of the RNA-DNA duplex as well as by base pairing between both the 5' (minus-strong stop DNA) and 3' (genome) R regions. Reverse transcription continues, moving from the $3^{\prime}$ end of the RNA genome towards the PBS and as polymerization occurs, RT-associated RNase $\mathrm{H}$ degrades the RNA template.

During the second stage of reverse transcription (44), as minus-strand DNA synthesis proceeds towards the PBS, the RNase $\mathrm{H}$ domain of RT makes a specific cleavage in the RNA genome at the PPT $(32,83)$. At this point, the minus strand can be 
used as a template in order to synthesize plus-strand strong stop DNA that copies the U3 R U5 (LTR) sequence as well as a region of the tRNA. The plus-strand strong stop DNA is transferred to the $5^{\prime}$ end of the minus strand and hybridizes to a complementary PBS on the minus-strand DNA. Once this has occurred, DNA synthesis proceeds in both directions producing the dsDNA intermediate with LTRs at both ends. RNase $\mathrm{H}$ removes the tRNA primer and the viral integrase inserts the dsDNA into the host's chromosome.

Reverse transcriptase. The process of reverse transcription relies on the viral enzyme RT, an RNA- and DNA-dependent DNA polymerase with associated RNase H activity. Due to the central role RT plays in the retroviral life cycle, many of its amino acid motifs are strictly conserved, even among divergent retroviral species (80). This observation can be expanded to the RTs of retroelements as well as to the structurally similar cellular enzyme, telomerase $(72,80)$. Despite the low primary sequence homology between these polymerases, sequences such as the dNTP binding pocket including the Thr-Val-Leu-Asp (TVLD) sequence, the Leu-Pro-Gln-Gly (LPQG) motif, and the Tyr-X-Asp-Asp (YXDD) box are highly conserved among RTs, therefore hinting at critical roles that these amino acids partake in proper polymerase function. Previous studies analyzing solvent accessibility have provided data placing the residues of these motifs in close proximity to the active site of RT (96).

Crystal structure of HIV-1 RT. The crystal structure of HIV-1 RT complexed with a non-nucleoside inhibitor at a resolution of 3.5 Angstroms, shows that the enzyme is a highly elongated, asymmetric heterodimer composed of two polypeptides, 66 and $51 \mathrm{kD}$, respectively. A simplistic three-dimensional view of HIV-1 RT has been compared to a right hand (Fig. 4 and 5A) $(47,49,57,96)$. The p66 subunit can be 
separated into five domains that includes the fingers, thumb, palm, connection and the RNase H domains $(20,49,57)$. The fingers, palm and connection domains are composed of several mixed $\beta$-sheets and $\alpha$-helices whereas the thumb domain is characterized as a 4 $\alpha$-helix bundle. The p51 subunit is a truncated version of p66 lacking the RNase $\mathrm{H}$ domain. The overall tertiary structure of the p51 subunit domains is similar to the p66 subunit, yet the spatial arrangements of these domains differ between the two subunits $(49,57)$. Both subunits contain a polymerase active site found in the palm domain, yet only the one present in p66 is active. Finally, a nucleic acid binding cleft runs between the polymerase active site and the RNase H domain (57).

The crystal structures of several ternary complexes of HIV-1 RT have been elucidated providing structural information on how the protein may function and interact with its substrates (Fig. 5A and B) (47, 49). In one study, HIV-1 RT has been crystallized with dsDNA template-primer at a resolution of 3.0 Angstroms (49). It was observed in this model that several interactions can occur between the fingers, thumb and palm domains of the protein with the sugar-phosphate backbone of the nucleic acid. This involves both the $\alpha \mathrm{H}$ and $\alpha \mathrm{I}$ helices of the thumb domain that bind to the primer and template backbones, respectively. Further interactions between RT and primer may involve the $\beta 12-13$ hairpin (primer grip) and regions including portions of the fingers, thumb and palm (template grip) may position the template. In addition, the dsDNA was shown to adopt both $\mathrm{A}$ and $\mathrm{B}$ forms that were separated by a $40-45^{\circ}$ bend. The significance of these adopted forms of DNA and RNA may influence cleavage events by the RNase H domain (3). 
Recently, a ternary complex of HIV-1 RT with template-primer and deoxythymidine triphosphate (dTTP) was determined, implicating several residues in the direct binding of the incoming deoxyribonucleotide triphosphate (dNTP) (47). These residues include K65, R72, D113, A114, Y115, and Q151, located in the dNTP binding pocket of HIV-1 RT (Fig. 5B). The side-chains of both K65 and R72 as well as the mainchain amides of D113 and A114 interact with the triphosphate moiety of incoming dTTP through hydrogen bonding. In addition, the R72 residue interacts with the base of the incoming substrate. Mutational analysis of these positions in HIV-1 RT has revealed information implicating these residues with positional effects on the template-primer complex, conformational changes of RT as well as pyrophosphate exchange $(47,61,89$, 96). Residues Y115 and Q151 side-chains were also shown to interact with the base of the dTTP substrate. The aromatic ring of the Y115 is in close proximity to the ribose moiety and may play a role in the selectivity of dNTPs versus ribonucleotides (rNTPs).

Comparison of these crystal structures and a closer inspection of the active site of HIV-1 RT places the terminal 3' hydroxyl group of the primer in close proximity to residues of the YMDD box $(47,49,57)$. Residues of this particular motif, specifically the Tyr and Met (Y183 and M184), have been implicated with catalytic function, infectivity potential, positioning of template-primer complex, processivity, and fidelity $(5,13,96$, 106). In addition, the two Asp residues (D185 and 186) in accordance with Asp (D110) of the dNTP binding pocket are believed to coordinate magnesium $\left(\mathrm{Mg}^{2+}\right)$ important in the metal-mediated nucleotidyltransferase reaction $(61,62)$.

Several crystal structures of unliganded HIV-1 RT have been resolved revealing difference between both unliganded and complexed enzyme $(30,45,47,49,57)$. Both 
subtle and major structural changes have been observed when comparing these structures. One of the more profound changes involves the thumb, which is characterized by a $30^{\circ}$ displacement when comparing the structures of HIV-1 RT in the absence or presence of DNA. Interestingly, the geometry of the active site seems to be unchanged in the presence or absence of this substrate.

Crystal structure of MLV RT. The crystal structure of a catalytically active MLV RT fragment containing the fingers and palm domains has been determined at a resolution of 1.8 Angstroms. In addition, the crystal structure of this catalytic fragment complexed with a $\mathrm{Mn}^{2+}$ cation was also determined at a resolution of 2.6 Angstroms (Fig. 6) (36). The structure reveals that the fingers and palm domains of this crystallized fragment are composed of $\alpha$ helices and $\beta$ sheets. In a junction between these fingers and palm domains resides the $\beta_{10}-\beta_{11}$ turn that includes the highly conserved YVDD box. The amino acids of this motif and those of the putative dNTP-binding site are believed to create the active site of the polymerase. This includes the catalytic triad (D150, D224, and $\mathrm{D} 225$ ) that has been observed to coordinate $\mathrm{Mn}^{2+}$ and which may play a critical role in catalysis.

Sequence alignments of MLV and HIV-1 RTs reveal that both polymerases share many of the highly conserved motifs (TVLD, LPQG and YXDD boxes) and possess similar tertiary structures despite only a $25 \%$ amino acid identity $(36,80)$. In addition, this sequence alignment suggests that residues K103, R110, D153, A154, F155, and Q190 in MLV RT are equivalent to residues K65, R72, D113, A114, Y115, and Q151 in HIV-1 RT, respectively as described in Chapter 3. Furthermore, the HIV-1 RT equivalents have been observed to directly contact the incoming dTTP substrate (47). 
The involvement of these MLV RT residues (K103, R110, D153, A154, F155, and Q190) in binding of incoming dNTP is supported by previous studies implicating residues like K103 and F155 of MLV RT with dNTP binding and selectivity, respectively $(6,34,36)$. In contrast, MLV RT possesses a number of additional features absent from HIV-1 RT including 40 amino acids at the $\mathrm{N}$-terminal end as well as several additional $\beta$ sheets and $\alpha$ helices. Finally, the crystallized fragment of MLV RT is accompanied by subtle changes in both the fingers and palm domains when compared to HIV-1 RT $(36,45)$.

Fidelity associated with reverse transcriptase. An important property of polymerases is fidelity, defined as the accuracy the enzyme displays in copying a template. The fidelity of a polymerase can be measured as the mutation rate, the rate at which mutations are introduced during synthesis of nascent DNA. Fidelity of polymerases, including RTs, can be measured in vitro or in vivo employing the use of either reversion or forward mutation assays.

The reversion assay, characterized by an inactivated reporter gene containing a substitution, measures the rate at which a functional gene is reconstituted. The rate of this genes reconstitution is equal to the mutation rate $(23,24)$. Limitations associated with this assay typically involve a small number of mutational targets providing measurements that are not indicative of the average mutation rate, since mutations can occur in a sequence dependent manner. In contrast, the forward mutation assay involves the use of a functional reporter gene and measures the rate at which this gene is inactivated by substitutions introduced during DNA synthesis. The frequency with which this inactivation occurs is set to equal the mutation rate $(63,64,74-76)$. This type of 
assay ordinarily allows for a larger number of mutational targets providing improved measurements of an average mutation rate in comparison to the reversion assay.

Since the retroviral life cycle employs the use of cellular DNA and RNA polymerases as well as RT, mutations contributing to genetic diversity can be introduced at several points of this life cycle from any one of these enzymes. Mutation rates of cellular DNA polymerases have been estimated to be on the order of $10^{-9}$ substitutions/bp/cycle of replication, an impressive feat involving highly processive DNA synthesis and the utilization of proof-reading mechanisms (58). Due to the highly accurate DNA synthesis exhibited by these polymerases, the effects on the overall mutation rate associated with retroviral replication is probably negligible. The mutation rate of RNA polymerase has not been determined and may contribute to the overall mutation rate, however, proofreading activity has been recently observed with this protein (101). Therefore, with the increased fidelity of this enzyme, its contribution to the overall mutation rate is likely minimal. It has been hypothesized that the major contributor of mutations introduced in the retroviral life cycle may be attributed to RT as a result of its less processive nature and the absence of proofreading activity $(7,100)$. Mutation rates of various RTs have been measured both in vivo and in vitro $(9,10,77)$. In vivo mutation rates of several RTs where shown to range between $3.4 \times 10^{-5}$ and 4.8 $\times 10^{-6}$ mutations/bp/replication cycle $(63,64,74-76)$, approximately 1,000 to 10,000 -fold more error-prone than the cellular DNA polymerases.

A comparison of mutation rates obtained by both in vitro and in vivo assays for HIV-1 RT reveals measurements varying by approximately 20 -fold $(9,63)$. These discrepancies, at least in the case of the cell-free in vitro assays utilizing purified RT, 
dNTPs and exogenous template-primer complexes, may be attributed to several factors including, both $\mathrm{Mg} 2+$ and dNTPs concentrations as well as $\mathrm{pH}(14,27,65,104)$. In addition, damage of polymerases during the purification process can also alter the fidelity of these enzymes $(14,58,84)$. Therefore, in light of these differences, there is a need to analyze the fidelity of RTs in vivo, within the context of a replicating reverse transcriptase complex. This may provide a more appropriate assessment of how RT fidelity is manifested in a physiological setting.

Structural determinants of RT important for fidelity. As illustrated previously (Fig. 4, 5A, 5B, and 6), the tertiary structure of various RTs resembles a right hand with fingers, palm and thumb domains $(36,47,49,57)$. Interactions between the RT (fingers, thumb, and palm domains) and substrates (template-primer and dNTP), may result in correct positioning of these substrates in a fashion that promotes polymerization. Collectively, substitutions in any of these domains may exert effects on the overall fidelity of the polymerase. In support of this hypothesis, several amino acids and/ or structural motifs in various RTs have been observed in vitro to be important for fidelity $(5,8,11,25,26,43,52,56,66,67,73,86,97,107)$. For example, the Y183F and M184V mutations in the YMDD motif of HIV-1 RT displayed enhanced fidelity compared to wild type $(5,25,73,105)$. Similar results were observed with the Y222F mutant of MLV RT (52). Mutations L74V, D76V, E89G, and F160W introduced in the fingers and palm domains of HIV-1 RT also were associated with increased fidelities (25, 42, 43, 56, 87). This was also evident in HIV-2 RT, at least for the L74V and E89G mutants (97). Finally, mutations introduced in the primer-grip were associated with significant increases in fidelity in vitro (107). In addition to increased fidelities, mutants 
like K65R and Q151M exhibited reductions in sensitivity to a number of dideoxynucleoside analogs $(38,85)$. Conversely, increases either in frameshifts, misincorporations, or mispair extensions resulted when substitutions were introduced either at residue Y115 (palm) or the $\alpha \mathrm{H}$ helix (thumb) in HIV-1 RT $(8,11,66,67$ ). Although, evidence has been compiled for determinants important for fidelity in vitro, amino acids and motifs responsible for fidelity in vivo are currently unknown.

Fidelity of reverse transcriptase and drug-resistance. Low RT fidelity leads to extensive genetic heterogeneity and drug-resistant viruses that arise impede efforts to combat retroviral infections. Various inhibitors have been designed targeting several different steps in the retroviral life cycle. These inhibitors fall into three classes; those that target RT, PR or binding/fusion interactions between virus and host (17). In the case of HIV-1 infections, at least 14 antiretroviral drugs directed against RT and PR are being utilized today in a clinical setting to hinder the spread of the virus and slow the progression to $\operatorname{AIDS}(18,98)$. RT inhibitors are categorized as nucleoside, nonnucleoside, or pyrophosphate analogs $(17,96)$. The nucleoside analogs were among the first exploited RT inhibitors observed to inhibit reverse transcription $(4,17)$. Several examples of nucleoside analogs include $2^{\prime}, 3^{\prime}$-dideoxy-3'-thiacytidine (3TC), 3'-azido-3'deoxythymidine (AZT), 2',3'-dideoxycytidine (ddC), 2',3'-dideoxyinosine (ddI), and $2^{\prime}, 3^{\prime}$-didehydro-3'-deoxythimidine (d4T). Compelling data from various studies suggest that combinations of these RT inhibitors with other antiretroviral inhibitors can prolong life expectancies and delay the progression of $\operatorname{AIDS}(19,33,40,41,70,71)$. However, prolonged treatment with antiviral drugs eventually results in the generation of drugresistant mutants in both patients and tissue culture-based systems $(35,39,53,60,90$, 
102). For example, within the YMDD motif of HIV-1 RT, the M184V, M184I, or the M184T mutations are associated with $3 \mathrm{TC}$ resistance $(35,90,102)$. In addition, similar mutations are observed in the homologous amino acid positions of the simian immunodeficiency virus (SIV), feline immunodeficiency virus (FIV), and hepatitis B virus (HBV), suggesting a common mechanism through which the enzyme acquires mutations that confer resistance $(2,15,93)$. Contrary to the single amino acid substitution associated with $3 \mathrm{TC}$ resistance, AZT resistance requires the introduction of several mutations in and around the active site of HIV-1 RT (53, 60). This resistant phenotype is characterized by the M41L, D67N, K70R, T215Y/F, and K219Q mutations. Furthermore, a number of mutations in HIV-1 RT result in cross-resistance to several nucleoside analogs simultaneously $(35,39,54,82,91,92,94)$. For instance, both the M184V and L74V mutations have been shown to be cross-resistant to ddC and ddI (35, 39, 94). Also, the K65R, E89G, and G333E mutations all exhibit a resistance to 3TC in addition to ddC, phosphonoformic acid, or AZT, respectively $(39,54,82)$. Finally, the multi-drug resistant mutation Q151M confers resistance to AZT, ddI or ddC $(91,92)$.

Elucidation of the ternary complex of HIV-1 RT with template-primer and dTTP substrates reveals that not all mutations associated with drug resistance are located within the active site of the polymerase (47). Interestingly, drug-resistant mutations to nucleoside analogs can fall into two categories. The first group includes mutations that reside in and around the dNTP binding site and these changes alone are sufficient to display this drug-resistant phenotype through mechanisms involving steric hinderance or alterations in the nature of drug binding $(31,47,88)$. This includes a number of positions in HIV-1 RT such as R72, L74, Q151, and M184 that directly contact either the template- 
primer complex or the incoming dNTP. For instance, L74 holds the templating base, M184 interacts with the sugar-moiety (3' nucleotide in primer), where as R72 and Q151 interact with the base of the incoming dNTP. Therefore, it is possible that substitutions at any of these positions can cause changes that eventually shift positioning of the incoming dNTP and alter catalysis or the nature of substrate binding. In contrast to mutations situated in the active site, the second set involves modifications located away from this pocket. These mutations may reinforce the changes associated with the drug-resistant mutations of the active site and realign critical residues important for proper function of the enzyme (47).

A comparative analysis of HIV-1 and MLV RTs demonstrates a number of similarities at both their primary and tertiary levels, despite only an $\sim 25 \%$ sequence homology $(36,57,80)$. In addition, the in vivo mutation rates of these two viruses are within 2-fold (77). Furthermore, MLV RT exhibits a drug sensitivity profile similar to HIV-1 RT $(50,95)$. This is characterized by sensitivities to several nucleoside analogs such as ddI, ddC, d4T, and AZT. Because of these similarities shared between the two polymerases, an MLV-based system serves as an ideal model for HIV-1, enabling us to gain insight into common mechanisms associated with fidelity and the development of drug-resistance. Similarities as well as differences between the RTs may also provide insights into retroviral evolution.

Overview of thesis. The fidelity of RT may be the major contributor of variation in retroviral populations, increasing the genetic diversity of the virus and eventually thwarting drug therapy. In order to understand the underlining mechanism by which fidelity is determined, it is critical to identify structural determinants of RTs that are 
important for fidelity in vivo. In Chapter 2, the development of an in vivo assay to identify structural determinants in MLV RT important for fidelity is discussed. Initially, I developed a rapid assay based on the ANGIE P encapsidating cell line that is sensitive enough to detect small but statistically significant differences in fidelity between mutants and wild type RT. Furthermore, I prove that the V223 position of MLV RT is important for fidelity in vivo. In Chapter 3, the effects of substitutions introduced at residues constituting the dNTP binding site of MLV RT are discussed in correlation to fidelity. I demonstrate, through extensive mutational analysis, that residues in MLV RT hypothesized to come in direct contact with the dNTP substrate are critical for proper polymerase function and viral replication. In addition, I show that several substitutions at these positions exhibit changes in fidelity and reveal that several residues that do not contact the dNTP can also have profound effects on fidelity in comparison to wild type RT. In Chapter 4, the resistance of both wild type and mutant MLV RTs to the nucleoside analog 3TC are discussed. I show that several V223 mutants of MLV RT as well as the wild type are all resistant to 3TC. Finally, in Chapter 5, I discuss evolution of retroviruses and explain the impending implications of my studies. 
FIG. 1. Retroviral genomic structure of MLV. Both RNA (top) and DNA (bottom) forms contain the genes $g a g$, pol, and env. The gag gene codes for structural proteins such as matrix, capsid, and nucleocapsid, where as pol codes for proteins utilized during replication such as reverse transcriptase and integrase. The env gene codes for the envelope glycoproteins that the virus utilizes during entry into host. Between gag and pol is pro, which codes for the viral protease important during assembly and maturation of virions. In addition to the coding regions, both the RNA and DNA genomes possess a number of cis-acting elements designated as the primer binding site (PBS), the packaging signal $(\Psi)$, and the polypurine tract (PPT). The Viral RNA possesses unique $5^{\prime}$ and $3^{\prime}$ sequences designated as U5 and U3 as well as an inverted repeats termed R. The unique sequences are duplicated in the DNA form of the retroviral genome and designated as the long terminal repeat (LTR). 


\section{Viral RNA}

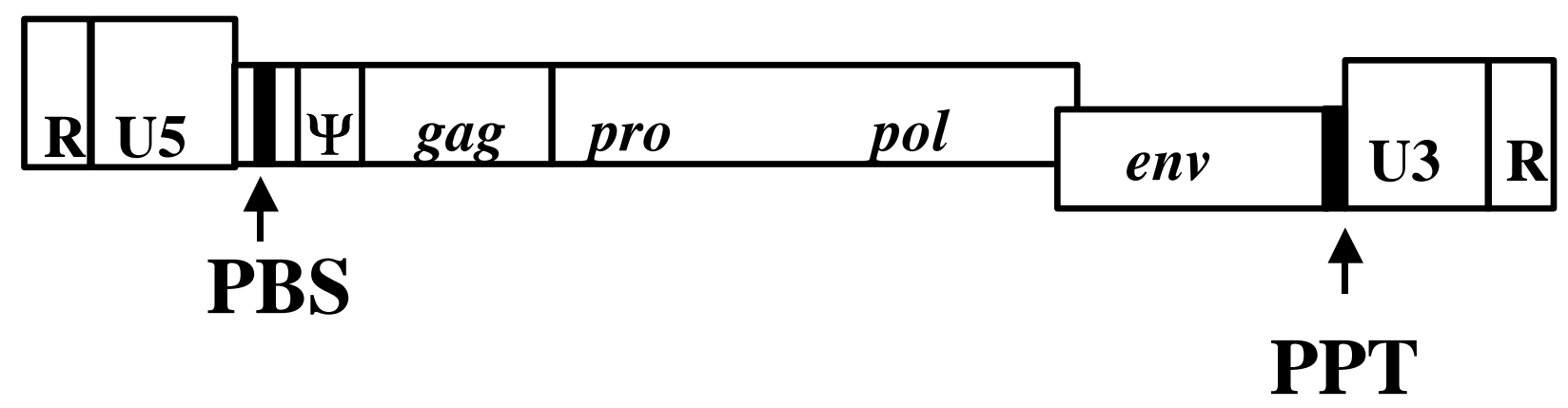

Proviral DNA

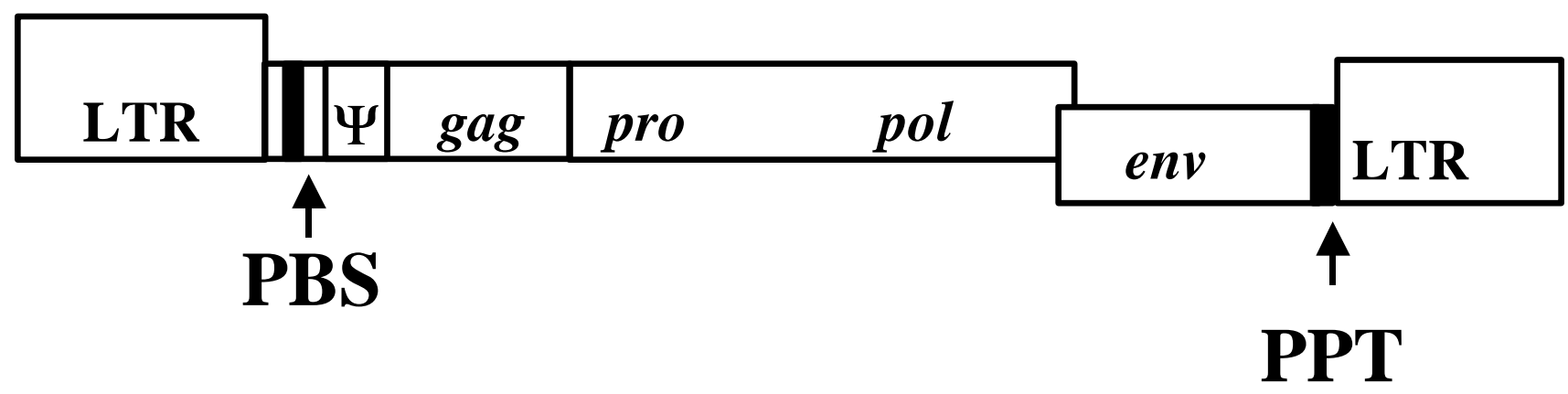

Figure 1. Retroviral Genomic 
FIG. 2. The retroviral lifecycle. Viral particle binds to cellular receptor, is internalized, and particle is uncoated. The RNA genome is reverse transcribed into dsDNA, which translocates to the nucleus, it integrates into host genome, and the host's RNA polymerase transcribes the provirus into mRNA. Full-length or spliced mRNA exported to the cytoplasm can be translated into protein or packaged into assembling viral particles. Assembled particles bud from the host and can repeat cycle. 


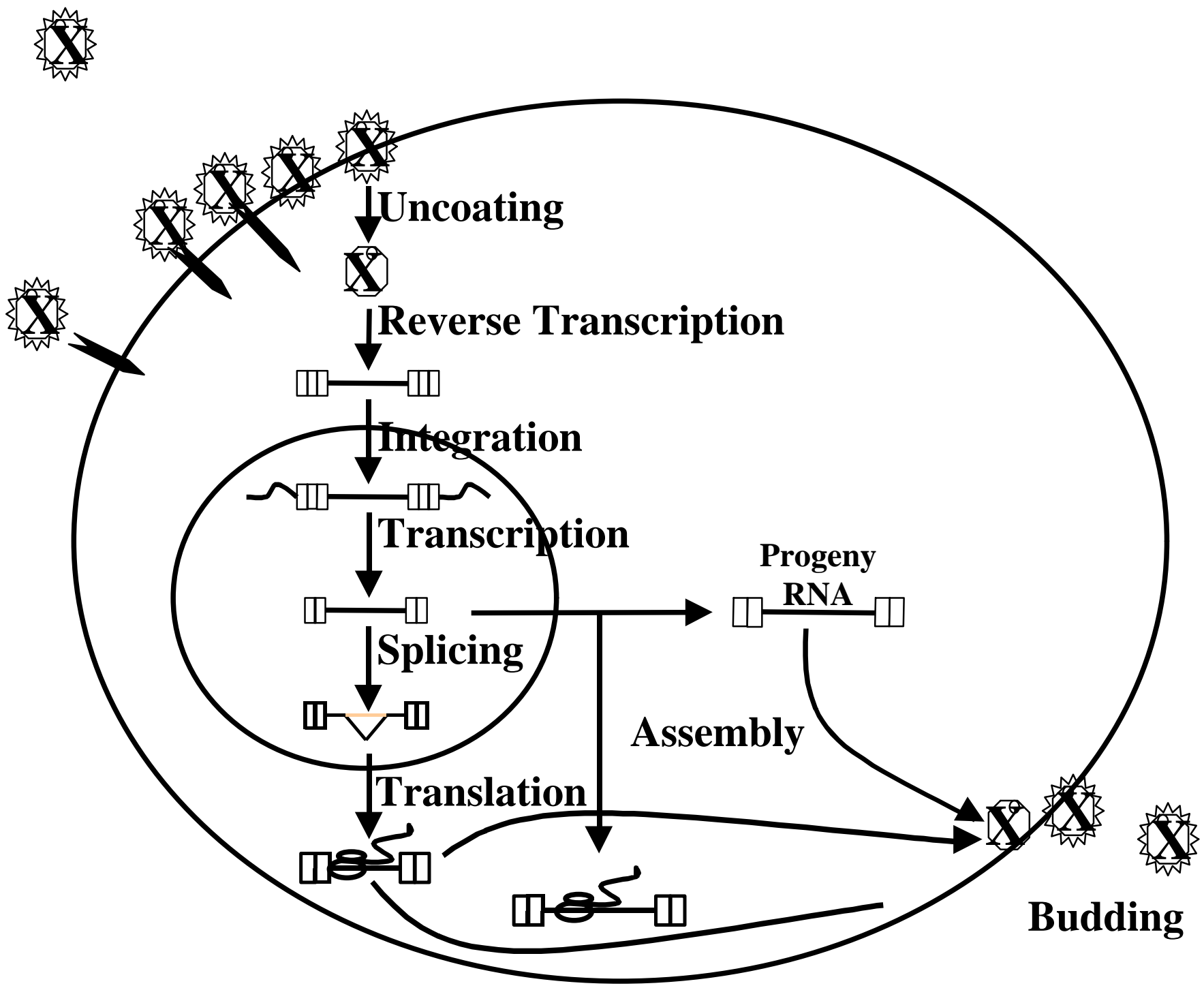

Figure 2. Retroviral Life 
FIG. 3. Reverse transcription. Thin lines represent viral RNA showing primer binding site (PBS), polypurine tract (PPT), unique $5^{\prime}$ and $3^{\prime}$ sequences (U5 and U3), as well as the inverted repeat $(\mathrm{R})$. Thick lines represent DNA and dotted lines RNA degradation by the RNase $\mathrm{H}$ domain. The crooked line represents the tRNA primer. 
1. $\frac{A}{\text { R U5 PBS }} \mid$ PPT U3 R $_{\mid}$

2.

3.

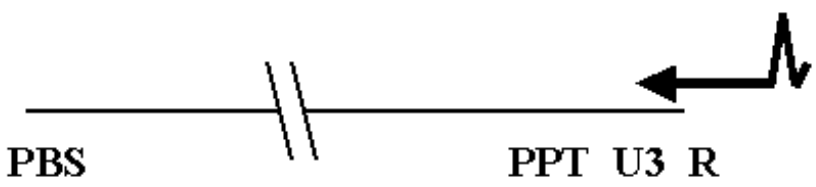

4.

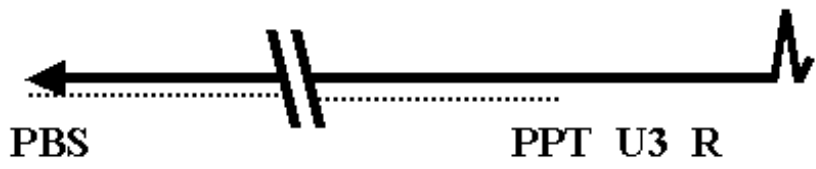

5.

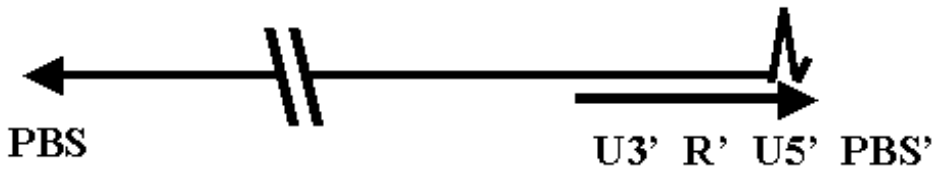

6.

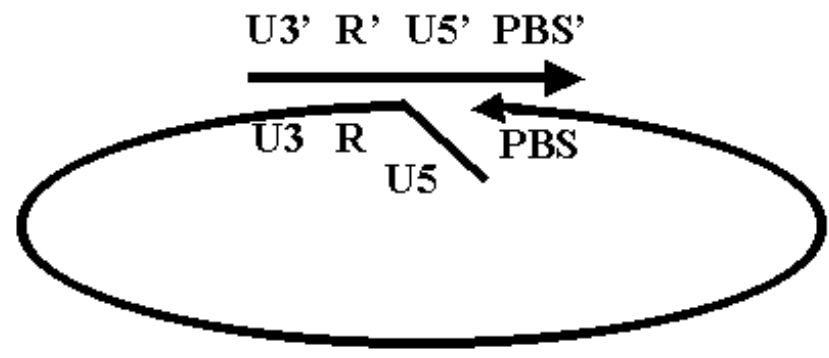

7.

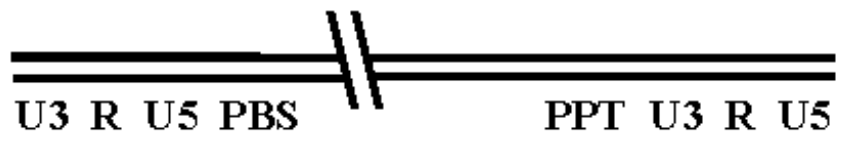

Figure 3. Steps in reverse transcription. Thin lines, RNA; thick lines, DNA; dotted lines, RNase $\mathrm{H}$ degradation; crooked line, tRNA primer. 
FIG. 4. Crystal Structure of HIV-1 Reverse Transcriptase Heterodimer

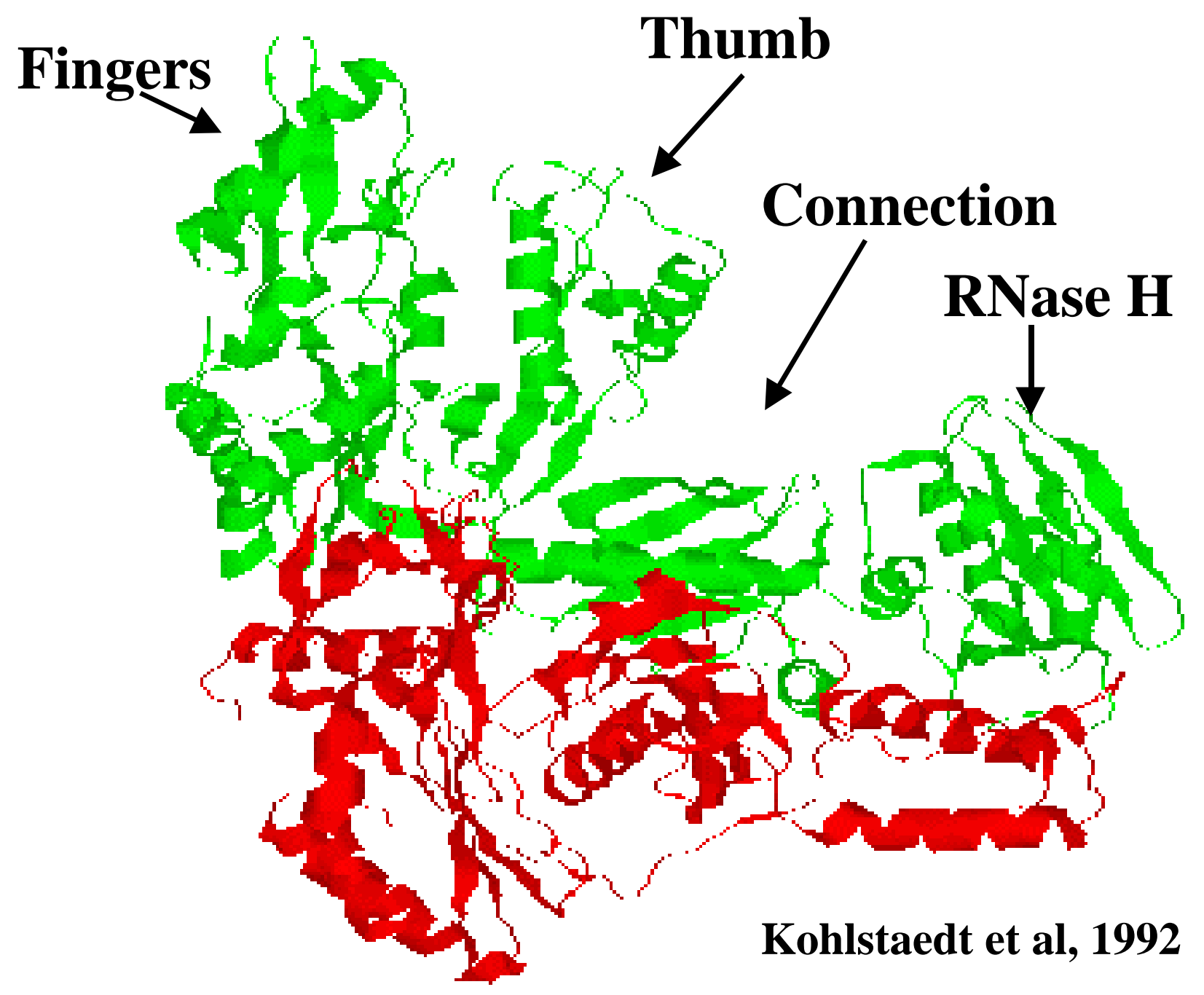

p66 Subunit

p51 Subunit 
FIG. 5. HIV-RT complexed with bound ligands. (A) Heterodimer of HIV-1 RT complexed with template-primer complex [Jacobo-Molina, et al 1994]. The structure contains both the p66 and p51 subunits of HIV-1 RT with a bound DNA substrate. (B) The dNTP-binding site of HIV-1 RT [Huang, et al 1998]. The incoming dNTP is characterized as box $(\mathrm{B}=$ base $)$, pentagon $(\mathrm{R}=$ ribose sugar $)$, and three diamonds $(\mathrm{P}=$ phosphates). The residue of HIV-1 RT shown to directly contact the incoming dNTP include the side chains of K65, R72, Y115, and Q151 as well as the main chain (mc) of D113 and A114). Contacts between residues and dNTP substrate are shown as dotted lines whereas the magnesium atom at site $\mathrm{B}$ is designated as $\mathrm{Mg}$. 


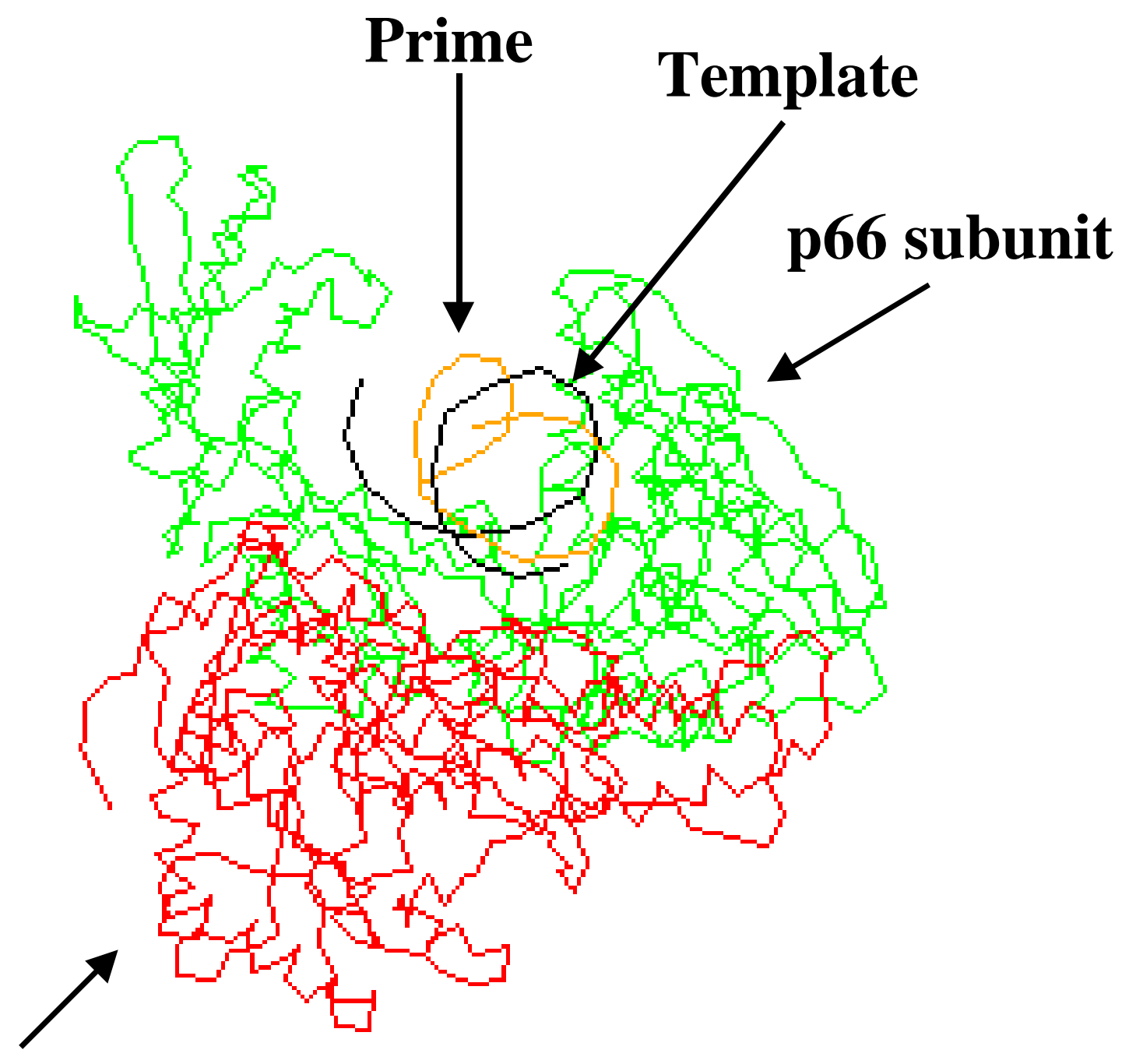

p51 subunit

Jacobo-Molina et al, 1994

Figure 5A. HIV-1RT complexed with DNA 


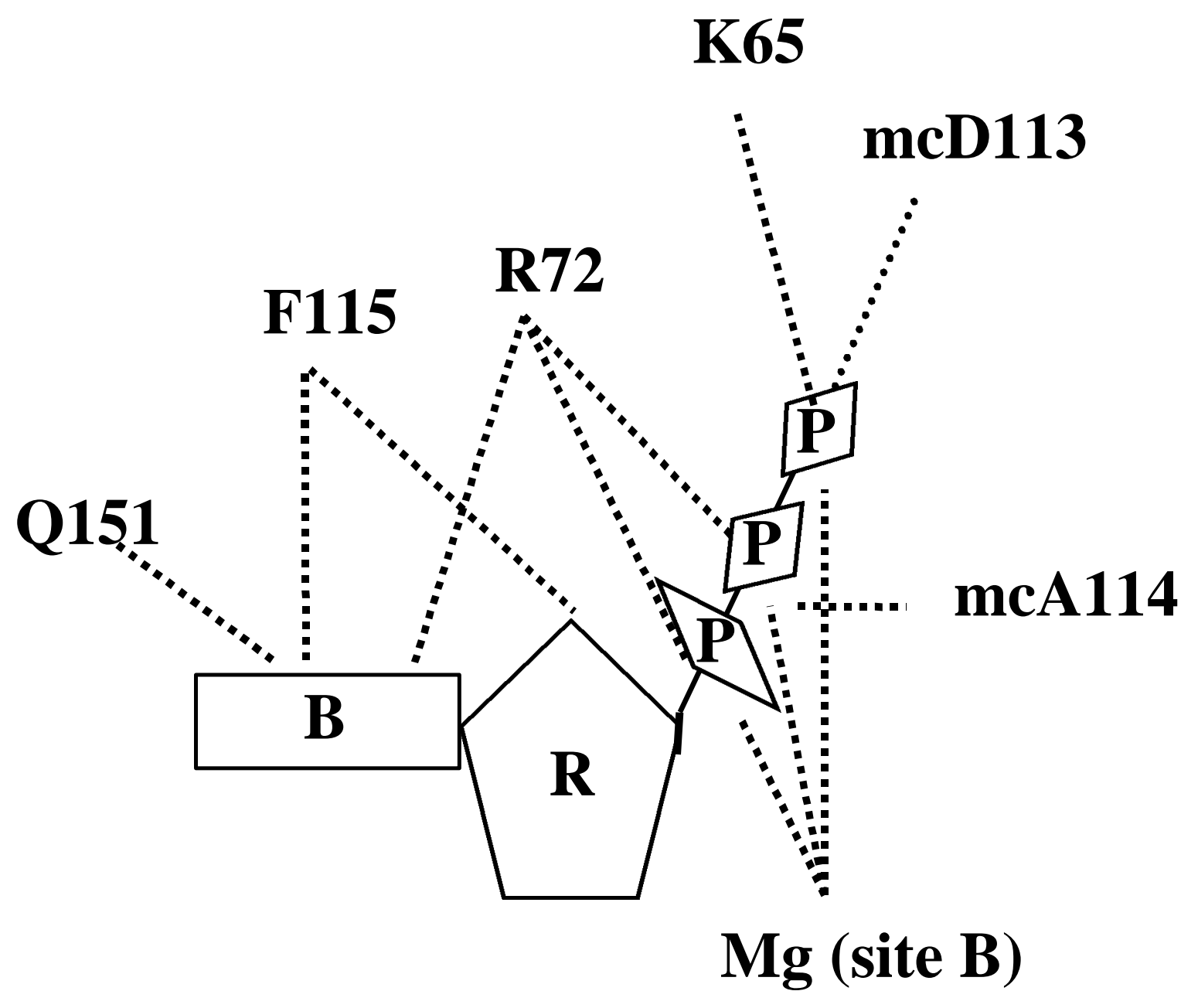

Huang et al, 1998

Figure 5B. HIV-1 RT dNTP Binding Site 
FIG. 6. The crystal structure of a catalytically active fragment of MLV RT [Georgiadis, et al 1995]. The structure includes the fingers and palm domains of MLV RT. The residues hypothesized to contact the incoming dNTP substrate are highlighted in black, which includes K103, R110, D153, A154, F155, and Q190. The location of both the YVDD box and the primer grip are also highlighted. 


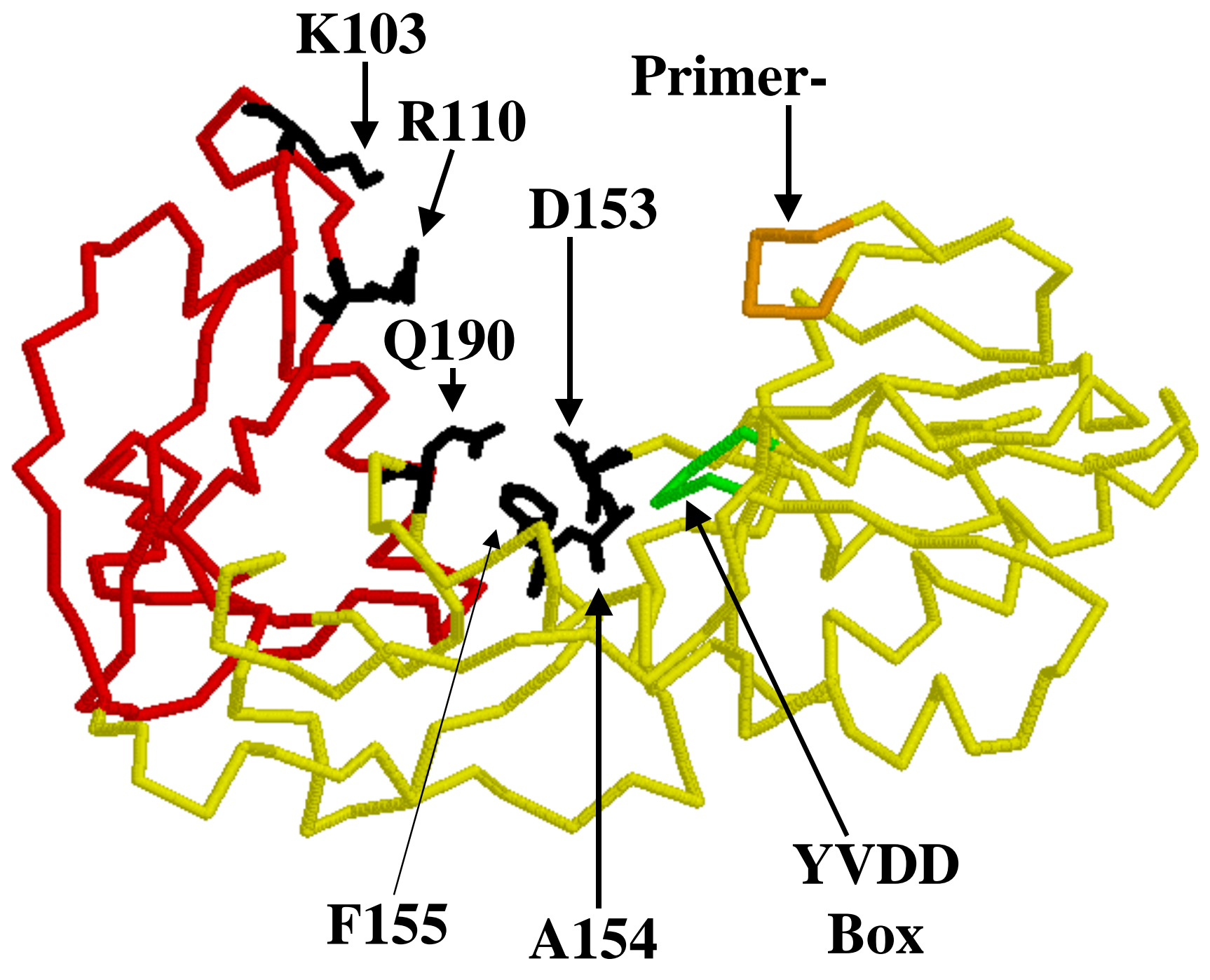

Georgiadis et al, 1995

Figure 6. Crystal Structure of MLV RT. 


\section{REFERNCES}

1. Alexander, F., J. Leis, D. A. Soltis, R. M. Crowl, W. Danho, M. S. Poonian, Y. C. Pan, and A. M. Skalka. 1987. Proteolytic processing of avian sarcoma and leukosis viruses pol-endo recombinant proteins reveals another pol gene domain. J. Virol. 61:534-542.

2. Allen, M. I., M. Deslauriers, C. W. Andrews, G. A. Tipples, K. A. Walters, D. L. Tyrrell, N. Brown, and L. D. Condreay. 1998. Identification and characterization of mutations in hepatitis $\mathrm{B}$ virus resistant to lamivudine. Lamivudine Clinical Investigation Group. Hepatology 27:1670-1677.

3. Arnold, E., J. Ding, S. H. Hughes, and Z. Hostomsky. 1995. Structures of DNA and RNA polymerases and their interactions with nucleic acid substrates. Curr. Opin. Struct. Biol. 5:27-38.

4. Arts, E. J., and M. A. Wainberg. 1996. Mechanisms of nucleoside analog antiviral activity and resistance during human immunodeficiency virus reverse transcription. Antimicrob. Agents Chemother. 40:527-540.

5. Bakhanashvili, M., O. Avidan, and A. Hizi. 1996. Mutational studies of human immunodeficiency virus type 1 reverse transcriptase: the involvement of residues 183 and 184 in the fidelity of DNA synthesis. FEBS Lett. 391:257-262.

6. Basu, A., V. B. Nanduri, G. F. Gerard, and M. J. Modak. 1988. Substrate binding domain of murine leukemia virus reverse transcriptase. Identification of lysine 103 and lysine 421 as binding site residues. J. Biol. Chem. 263:1648-1653. 
7. Battula, N., and L. A. Loeb. 1976. On the fidelity of DNA replication. Lack of exodeoxyribonuclease activity and error-correcting function in avian myeloblastosis virus DNA polymerase. J. Biol. Chem. 251:982-986.

8. Beard, W. A., K. Bebenek, T. A. Darden, L. Li, R. Prasad, T. A. Kunkel, and S. H. Wilson. 1998. Vertical-scanning mutagenesis of a critical tryptophan in the minor groove binding track of HIV-1 reverse transcriptase. Molecular nature of polymerase-nucleic acid interactions. J. Biol. Chem. 273:30435-30442.

9. Bebenek, K., J. Abbotts, J. D. Roberts, S. H. Wilson, and T. A. Kunkel. 1989. Specificity and mechanism of error-prone replication by human immunodeficiency virus-1 reverse transcriptase. J. Biol. Chem. 264:16948-16956.

10. Bebenek, K., J. Abbotts, S. H. Wilson, and T. A. Kunkel. 1993. Error-prone polymerization by HIV-1 reverse transcriptase. Contribution of template-primer misalignment, miscoding, and termination probability to mutational hot spots. J. Biol. Chem. 268:10324-10334.

11. Bebenek, K., W. A. Beard, J. R. Casas-Finet, H. R. Kim, T. A. Darden, S. H. Wilson, and T. A. Kunkel. 1995. Reduced frameshift fidelity and processivity of HIV-1 reverse transcriptase mutants containing alanine substitutions in helix $\mathrm{H}$ of the thumb subdomain. J. Biol. Chem. 270:19516-19523.

12. Bohnlein, E., J. W. Lowenthal, M. Siekevitz, D. W. Ballard, B. R. Franza, and W. C. Greene. 1988. The same inducible nuclear proteins regulates mitogen activation of both the interleukin-2 receptor-alpha gene and type 1 HIV. Cell 53:827-836. 
13. Boyer, P. L., and S. H. Hughes. 1995. Analysis of mutations at position 184 in reverse transcriptase of human immunodeficiency virus type 1. Antimicrob. Agents Chemother. 39:1624-1628.

14. Brosius, S., F. Grosse, and G. Krauss. 1983. Subspecies of DNA polymerase alpha from calf thymus with different fidelity in copying synthetic templateprimers. Nucleic Acids Res. 11:193-202.

15. Cherry, E., M. Slater, H. Salomon, E. Rud, and M. A. Wainberg. 1997. Mutations at codon 184 in simian immunodeficiency virus reverse transcriptase confer resistance to the (-) enantiomer of 2',3'-dideoxy- 3'-thiacytidine. Antimicrob. Agents Chemother. 41:2763-2765.

16. Cobrinik, D., L. Soskey, and J. Leis. 1988. A retroviral RNA secondary structure required for efficient initiation of reverse transcription. J. Virol. 62:3622-3630.

17. Coffin, J., M., S. H. Hughes, and H. E. Varmus. 1997. Retroviruses, Cold Spring Harbor Press, Cold Spring Harbor, N. Y.

18. Cohen, J. 1997. The daunting challenge of keeping HIV suppressed. Science 277:32-33.

19. Cooley, T. P., L. M. Kunches, C. A. Saunders, J. K. Ritter, C. J. Perkins, C. McLaren, R. P. McCaffrey, and H. A. Liebman. 1990. Once-daily administration of 2',3'-dideoxyinosine (ddI) in patients with the acquired immunodeficiency syndrome or AIDS-related complex. Results of a Phase I trial. N. Engl. J. Med. 322:1340-1345. 
20. Davies, J. F. d., Z. Hostomska, Z. Hostomsky, S. R. Jordan, and D. A. Matthews. 1991. Crystal structure of the ribonuclease H domain of HIV-1 reverse transcriptase. Science 252:88-95.

21. Declercq, E., R. Mak, A. Stroobant, and L. A. Van Hemeldonck. 1993. [Epidemiology of AIDS in Europe]. Acta Urol. Belg. 61:327-335.

22. Domingo, E., E. Martinez-Salas, F. Sobrino, J. C. de la Torre, A. Portela, J. Ortin, C. Lopez-Galindez, P. Perez-Brena, N. Villanueva, R. Najera, and et al. 1985. The quasispecies (extremely heterogeneous) nature of viral RNA genome populations: biological relevance--a review. Gene 40:1-8.

23. Dougherty, J. P., and H. M. Temin. 1988. Determination of the rate of base-pair substitution and insertion mutations in retrovirus replication. J. Virol. 62:28172822.

24. Drake, J. W. 1993. Rates of spontaneous mutation among RNA viruses. Proc. Natl. Acad. Sci. USA 90:4171-4175.

25. Drosopoulos, W. C., and V. R. Prasad. 1998. Increased misincorporation fidelity observed for nucleoside analog resistance mutations M184V and E89G in human immunodeficiency virus type 1 reverse transcriptase does not correlate with the overall error rate measured in vitro. J. Virol. 72:4224-4230.

26. Drosopoulos, W. C., L. F. Rezende, M. A. Wainberg, and V. R. Prasad. 1998. Virtues of being faithful: can we limit the genetic variation in human immunodeficiency virus? J. Mol. Med. 76:604-612. 
27. Eckert, K. A., and T. A. Kunkel. 1993. Fidelity of DNA synthesis catalyzed by human DNA polymerase alpha and HIV-1 reverse transcriptase: effect of reaction pH. Nucleic Acids Res. 21:5212-5220.

28. Eigen, M. 1971. Selforganization of matter and the evolution of biological macromolecules. Naturwissenschaften 58:465-523.

29. Erickson, J. W., and S. K. Burt. 1996. Structural mechanisms of HIV drug resistance. Annu. Rev. Pharmacol. Toxicol. 36:545-571.

30. Esnouf, R., J. Ren, C. Ross, Y. Jones, D. Stammers, and D. Stuart. 1995. Mechanism of inhibition of HIV-1 reverse transcriptase by non- nucleoside inhibitors. Nat. Struct. Biol. 2:303-308.

31. Feng, J. Y., and K. S. Anderson. 1999. Mechanistic studies examining the efficiency and fidelity of DNA synthesis by the 3TC-resistant mutant (184V) of HIV-1 reverse transcriptase. Biochemistry 38:9440-9448.

32. Finston, W. I., and J. J. Champoux. 1984. RNA-primed initiation of Moloney murine leukemia virus plus strands by reverse transcriptase in vitro. J. Virol. 51:26-33.

33. Fischl, M. A., D. D. Richman, M. H. Grieco, M. S. Gottlieb, P. A. Volberding, O. L. Laskin, J. M. Leedom, J. E. Groopman, D. Mildvan, R. T. Schooley, and et al. 1987. The efficacy of azidothymidine (AZT) in the treatment of patients with AIDS and AIDS-related complex. A double-blind, placebocontrolled trial. N. Engl. J. Med. 317:185-191. 
34. Gao, G., and S. P. Goff. 1998. Replication defect of moloney murine leukemia virus with a mutant reverse transcriptase that can incorporate ribonucleotides and deoxyribonucleotides. J. Virol. 72:5905-5911.

35. Gao, Q., Z. Gu, M. A. Parniak, J. Cameron, N. Cammack, C. Boucher, and M. A. Wainberg. 1993. The same mutation that encodes low-level human immunodeficiency virus type 1 resistance to 2',3'-dideoxyinosine and 2',3'dideoxycytidine confers high-level resistance to the (-) enantiomer of 2',3'dideoxy-3'- thiacytidine. Antimicrob. Agents Chemother. 37:1390-1392.

36. Georgiadis, M. M., S. M. Jessen, C. M. Ogata, A. Telesnitsky, S. P. Goff, and W. A. Hendrickson. 1995. Mechanistic implications from the structure of a catalytic fragment of Moloney murine leukemia virus reverse transcriptase. Structure 3:879-892.

37. Gilboa, E., S. W. Mitra, S. Goff, and D. Baltimore. 1979. A detailed model of reverse transcription and tests of crucial aspects. Cell 18:93-100.

38. Gu, Z., E. J. Arts, M. A. Parniak, and M. A. Wainberg. 1995. Mutated K65R recombinant reverse transcriptase of human immunodeficiency virus type 1 shows diminished chain termination in the presence of $2^{\prime}, 3^{\prime}$-dideoxycytidine $5^{\prime}$ triphosphate and other drugs. Proc. Natl. Acad. Sci. USA 92:2760-2764.

39. Gu, Z., Q. Gao, H. Fang, H. Salomon, M. A. Parniak, E. Goldberg, J. Cameron, and M. A. Wainberg. 1994. Identification of a mutation at codon 65 in the IKKK motif of reverse transcriptase that encodes human immunodeficiency virus resistance to 2',3'-dideoxycytidine and 2',3'-dideoxy-3'-thiacytidine. Antimicrob. Agents Chemother. 38:275-281. 
40. Gulick, R. M., J. W. Mellors, D. Havlir, J. J. Eron, C. Gonzalez, D. McMahon, L. Jonas, A. Meibohm, D. Holder, W. A. Schleif, J. H. Condra, E. A. Emini, R. Isaacs, J. A. Chodakewitz, and D. D. Richman. 1998. Simultaneous vs sequential initiation of therapy with indinavir, zidovudine, and lamivudine for HIV-1 infection: 100-week follow-up. JAMA 280:35-41.

41. Gulick, R. M., J. W. Mellors, D. Havlir, J. J. Eron, C. Gonzalez, D. McMahon, D. D. Richman, F. T. Valentine, L. Jonas, A. Meibohm, E. A. Emini, and J. A. Chodakewitz. 1997. Treatment with indinavir, zidovudine, and lamivudine in adults with human immunodeficiency virus infection and prior antiretroviral therapy. N. Engl. J. Med. 337:734-739.

42. Gutierrez-Rivas, M., A. Ibanez, M. A. Martinez, E. Domingo, and L. Menendez-Arias. 1999. Mutational analysis of Phe160 within the "palm" subdomain of human immunodeficiency virus type 1 reverse transcriptase. J. Mol. Biol. 290:615-625.

43. Hamburgh, M. E., W. C. Drosopoulos, and V. R. Prasad. 1998. The influence of 3TC-resistance mutations E89G and M184V in the human immunodeficiency virus reverse transcriptase on mispair extension efficiency. Nucleic Acids Res. 26:4389-4394.

44. Hsieh, J. C., S. Zinnen, and P. Modrich. 1993. Kinetic mechanism of the DNAdependent DNA polymerase activity of human immunodeficiency virus reverse transcriptase. J. Biol. Chem. 268:24607-24613.

45. Hsiou, Y., J. Ding, K. Das, A. D. Clark, Jr., S. H. Hughes, and E. Arnold. 1996. Structure of unliganded HIV-1 reverse transcriptase at 2.7 A resolution: 
implications of conformational changes for polymerization and inhibition mechanisms. Structure 4:853-860.

46. Hsu, H. W., P. Schwartzberg, and S. P. Goff. 1985. Point mutations in the P30 domain of the gag gene of Moloney murine leukemia virus. Virology 142:211214.

47. Huang, H., R. Chopra, G. L. Verdine, and S. C. Harrison. 1998. Structure of a covalently trapped catalytic complex of HIV-1 reverse transcriptase: implications for drug resistance. Science 282:1669-1675.

48. Hunter, E., E. Hill, M. Hardwick, A. Bhown, D. E. Schwartz, and R. Tizard. 1983. Complete sequence of the Rous sarcoma virus env gene: identification of structural and functional regions of its product. J. Virol. 46:920-936.

49. Jacobo-Molina, A., J. Ding, R. G. Nanni, A. D. Clark, Jr., X. Lu, C. Tantillo, R. L. Williams, G. Kamer, A. L. Ferris, P. Clark, and et al. 1993. Crystal structure of human immunodeficiency virus type 1 reverse transcriptase complexed with double-stranded DNA at 3.0 A resolution shows bent DNA. Proc. Natl. Acad. Sci. USA 90:6320-6324.

50. Julias, J. G., T. Kim, G. Arnold, and V. K. Pathak. 1997. The antiretrovirus drug 3'-azido-3'-deoxythymidine increases the retrovirus mutation rate. J. Virol. 71:4254-4263.

51. Kane, S. E., and K. Beemon. 1985. Precise localization of m6A in Rous sarcoma virus RNA reveals clustering of methylation sites: implications for RNA processing. Mol. Cell. Biol. 5:2298-2306. 
52. Kaushik, N., K. Singh, I. Alluru, and M. J. Modak. 1999. Tyrosine 222, a member of the YXDD motif of MuLV RT, is catalytically essential and is a major component of the fidelity center. Biochemistry 38:2617-2627.

53. Kellam, P., C. A. Boucher, and B. A. Larder. 1992. Fifth mutation in human immunodeficiency virus type 1 reverse transcriptase contributes to the development of high-level resistance to zidovudine. Proc. Natl. Acad. Sci. USA 89:1934-1938.

54. Kemp, S. D., C. Shi, S. Bloor, P. R. Harrigan, J. W. Mellors, and B. A. Larder. 1998. A novel polymorphism at codon 333 of human immunodeficiency virus type 1 reverse transcriptase can facilitate dual resistance to zidovudine and L-2',3'-dideoxy-3'-thiacytidine. J. Virol. 72:5093-5098.

55. Kim, A., C. Terzian, P. Santamaria, A. Pelisson, N. Purd'homme, and A. Bucheton. 1994. Retroviruses in invertebrates: the gypsy retrotransposon is apparently an infectious retrovirus of Drosophila melanogaster. Proc. Natl. Acad. Sci. USA 91:1285-1289.

56. Kim, B., T. R. Hathaway, and L. A. Loeb. 1998. Fidelity of mutant HIV-1 reverse transcriptases: interaction with the single-stranded template influences the accuracy of DNA synthesis. Biochemistry 37:5831-5839.

57. Kohlstaedt, L. A., J. Wang, J. M. Friedman, P. A. Rice, and T. A. Steitz. 1992. Crystal structure at 3.5 A resolution of HIV-1 reverse transcriptase complexed with an inhibitor. Science 256:1783-1790. 
58. Kunkel, T. A., R. K. Hamatake, J. Motto-Fox, M. P. Fitzgerald, and A. Sugino. 1989. Fidelity of DNA polymerase I and the DNA polymerase I-DNA primase complex from Saccharomyces cerevisiae. Mol. Cell Biol. 9:4447-4458.

59. Lampson, B. C., J. Sun, M. Y. Hsu, J. Vallejo-Ramirez, S. Inouye, and M. Inouye. 1989. Reverse transcriptase in a clinical strain of Escherichia coli: production of branched RNA-linked msDNA. Science 243:1033-1038.

60. Larder, B. A., and S. D. Kemp. 1989. Multiple mutations in HIV-1 reverse transcriptase confer high-level resistance to zidovudine (AZT). Science 246:11551158.

61. Larder, B. A., D. J. Purifoy, K. L. Powell, and G. Darby. 1987. Site-specific mutagenesis of AIDS virus reverse transcriptase. Nature 327:716-717.

62. Lowe, D. M., V. Parmar, S. D. Kemp, and B. A. Larder. 1991. Mutational analysis of two conserved sequence motifs in HIV-1 reverse transcriptase. FEBS Lett. 282:231-234.

63. Mansky, L. M., and H. M. Temin. 1995. Lower in vivo mutation rate of human immunodeficiency virus type 1 than that predicted from the fidelity of purified reverse transcriptase. J. Virol. 69:5087-5094.

64. Mansky, L. M., and H. M. Temin. 1994. Lower mutation rate of bovine leukemia virus relative to that of spleen necrosis virus. J. Virol. 68:494-499.

65. Martinez, M. A., J. P. Vartanian, and S. Wain-Hobson. 1994. Hypermutagenesis of RNA using human immunodeficiency virus type 1 reverse transcriptase and biased dNTP concentrations. Proc. Natl. Acad. Sci. USA 91:11787-11791. 
66. Martin-Hernandez, A. M., E. Domingo, and L. Menendez-Arias. 1996. Human immunodeficiency virus type 1 reverse transcriptase: role of Tyr115 in deoxynucleotide binding and misinsertion fidelity of DNA synthesis. EMBO J. 15:4434-4442.

67. Martin-Hernandez, A. M., M. Gutierrez-Rivas, E. Domingo, and L. Menendez-Arias. 1997. Mispair extension fidelity of human immunodeficiency virus type 1 reverse transcriptases with amino acid substitutions affecting Tyr115. Nucleic Acids Res. 25:1383-1389.

68. McClure, M. A., M. S. Johnson, and R. F. Doolittle. 1987. Relocation of a protease-like gene segment between two retroviruses. Proc. Natl. Acad. Sci. USA 84:2693-2697.

69. McMichael, A. J., and R. E. Phillips. 1997. Escape of human immunodeficiency virus from immune control. Annu. Rev. Immunol. 15:271-296.

70. Merigan, T. C., G. Skowron, S. A. Bozzette, D. Richman, R. Uttamchandani, M. Fischl, R. Schooley, M. Hirsch, W. Soo, C. Pettinelli, and et al. 1989. Circulating p24 antigen levels and responses to dideoxycytidine in human immunodeficiency virus (HIV) infections. A phase I and II study. Ann. Intern. Med. 110:189-194.

71. Murray, H. W., K. E. Squires, W. Weiss, S. Sledz, H. S. Sacks, J. Hassett, A. Cross, R. E. Anderson, and L. M. Dunkle. 1995. Stavudine in patients with AIDS and AIDS-related complex: AIDS clinical trials group 089. J. Infect. Dis. 171 Suppl. 2:S123-130. 
72. Nakamura, T. M., G. B. Morin, K. B. Chapman, S. L. Weinrich, W. H. Andrews, J. Lingner, C. B. Harley, and T. R. Cech. 1997. Telomerase catalytic subunit homologs from fission yeast and human. Science 277:955-959.

73. Pandey, V. N., N. Kaushik, N. Rege, S. G. Sarafianos, P. N. Yadav, and M. J. Modak. 1996. Role of methionine 184 of human immunodeficiency virus type-1 reverse transcriptase in the polymerase function and fidelity of DNA synthesis. Biochemistry 35:2168-2179.

74. Parthasarathi, S., A. Varela-Echavarria, Y. Ron, B. D. Preston, and J. P. Dougherty. 1995. Genetic rearrangements occurring during a single cycle of murine leukemia virus vector replication: characterization and implications. J. Virol. 69:7991-8000.

75. Pathak, V. K., and H. M. Temin. 1990. Broad spectrum of in vivo forward mutations, hypermutations, and mutational hotspots in a retroviral shuttle vector after a single replication cycle: deletions and deletions with insertions. Proc. Natl. Acad. Sci. USA 87:6024-6028.

76. Pathak, V. K., and H. M. Temin. 1990. Broad spectrum of in vivo forward mutations, hypermutations, and mutational hotspots in a retroviral shuttle vector after a single replication cycle: substitutions, frameshifts, and hypermutations. Proc. Natl. Acad. Sci. USA 87:6019-6023.

77. Pathak, V. K. a. W.-S. Hu. 1997. "Might as Well Jump". Mutation rates, defective genome formation, and recombination. Seminars in Virology 8:141150. 
78. Pepinsky, R. B., and V. M. Vogt. 1984. Fine-structure analyses of lipid-protein and protein-protein interactions of gag protein p19 of the avian sarcoma and leukemia viruses by cyanogen bromide mapping. J. Virol. 52:145-153.

79. Phillips, R. E., S. Rowland-Jones, D. F. Nixon, F. M. Gotch, J. P. Edwards, A. O. Ogunlesi, J. G. Elvin, J. A. Rothbard, C. R. Bangham, C. R. Rizza, and et al. 1991. Human immunodeficiency virus genetic variation that can escape cytotoxic T cell recognition. Nature 354:453-459.

80. Poch, O., I. Sauvaget, M. Delarue, and N. Tordo. 1989. Identification of four conserved motifs among the RNA-dependent polymerase encoding elements. EMBO J. 8:3867-3874.

81. Poulet, F. M., P. R. Bowser, and J. W. Casey. 1994. Retroviruses of fish, reptiles, and mollusks, p.1-38. In Levy, J. A. (ed), The Retroviridae, vol. 3. Plenum Press, New York, N. Y.

82. Quan, Y., Z. Gu, X. Li, C. Liang, M. A. Parniak, and M. A. Wainberg. 1998. Endogenous reverse transcriptase assays reveal synergy between combinations of the M184V and other drug resistance-conferring mutations in interactions with nucleoside analog triphosphates. J. Mol. Biol. 277:237-247.

83. Rattray, A. J., and J. J. Champoux. 1989. Plus-strand priming by Moloney murine leukemia virus. The sequence features important for cleavage by RNase H. J. Mol. Biol. 208:445-456.

84. Reyland, M. E., and L. A. Loeb. 1987. On the fidelity of DNA replication. Isolation of high fidelity DNA polymerase-primase complexes by immunoaffinity chromatography. J. Biol. Chem. 262:10824-10830. 
85. Rezende, L. F., K. Curr, T. Ueno, H. Mitsuya, and V. R. Prasad. 1998. The impact of multidideoxynucleoside resistance-conferring mutations in human immunodeficiency virus type 1 reverse transcriptase on polymerase fidelity and error specificity. J. Virol. 72:2890-2895.

86. Rezende, L. F., W. C. Drosopoulos, and V. R. Prasad. 1998. The influence of 3TC resistance mutation M184I on the fidelity and error specificity of human immunodeficiency virus type 1 reverse transcriptase. Nucleic Acids Res. 26:3066-3072.

87. Rubinek, T., M. Bakhanashvili, R. Taube, O. Avidan, and A. Hizi. 1997. The fidelity of 3' misinsertion and mispair extension during DNA synthesis exhibited by two drug-resistant mutants of the reverse transcriptase of human immunodeficiency virus type 1 with Leu74-->Val and Glu89-->Gly. Eur. J. Biochem. 247:238-247.

88. Sarafianos, S. G., K. Das, A. D. Clark, Jr., J. Ding, P. L. Boyer, S. H. Hughes, and E. Arnold. 1999. Lamivudine (3TC) resistance in HIV-1 reverse transcriptase involves steric hindrance with beta-branched amino acids. Proc. Natl. Acad. Sci. USA 96:10027-10032.

89. Sarafianos, S. G., V. N. Pandey, N. Kaushik, and M. J. Modak. 1995. Sitedirected mutagenesis of arginine 72 of HIV-1 reverse transcriptase. Catalytic role and inhibitor sensitivity. J. Biol. Chem. 270:19729-19735.

90. Schinazi, R. F., R. M. Lloyd, Jr., M. H. Nguyen, D. L. Cannon, A. McMillan, N. Ilksoy, C. K. Chu, D. C. Liotta, H. Z. Bazmi, and J. W. Mellors. 1993. 
Characterization of human immunodeficiency viruses resistant to oxathiolanecytosine nucleosides. Antimicrob. Agents Chemother. 37:875-881.

91. Shafer, R. W., A. K. Iversen, M. A. Winters, E. Aguiniga, D. A. Katzenstein, and T. C. Merigan. 1995. Drug resistance and heterogeneous long-term virologic responses of human immunodeficiency virus type 1-infected subjects to zidovudine and didanosine combination therapy. The AIDS Clinical Trials Group 143 Virology Team. J. Infect. Dis. 172:70-78.

92. Shirasaka, T., M. F. Kavlick, T. Ueno, W. Y. Gao, E. Kojima, M. L. Alcaide, S. Chokekijchai, B. M. Roy, E. Arnold, R. Yarchoan, and et al. 1995. Emergence of human immunodeficiency virus type 1 variants with resistance to multiple dideoxynucleosides in patients receiving therapy with dideoxynucleosides. Proc. Natl. Acad. Sci. USA 92:2398-2402.

93. Smith, R. A., K. M. Remington, R. M. Lloyd, Jr., R. F. Schinazi, and T. W. North. 1997. A novel Met-to-Thr mutation in the YMDD motif of reverse transcriptase from feline immunodeficiency virus confers resistance to oxathiolane nucleosides. J. Virol. 71:2357-2362.

94. St. Clair, M. H., J. L. Martin, G. Tudor-Williams, M. C. Bach, C. L. Vavro, D. M. King, P. Kellam, S. D. Kemp, and B. A. Larder. 1991. Resistance to ddI and sensitivity to AZT induced by a mutation in HIV-1 reverse transcriptase. Science 253:1557-1559.

95. Stair, R. K., C. J. Nelson, and J. W. Mellors. 1991. Use of recombinant retroviruses to characterize the activity of antiretroviral compounds. J. Virol. 65:6339-6342. 
96. Tantillo, C., J. Ding, A. Jacobo-Molina, R. G. Nanni, P. L. Boyer, S. H. Hughes, R. Pauwels, K. Andries, P. A. Janssen, and E. Arnold. 1994. Locations of anti-AIDS drug binding sites and resistance mutations in the threedimensional structure of HIV-1 reverse transcriptase. Implications for mechanisms of drug inhibition and resistance. J. Mol. Biol. 243:369-387.

97. Taube, R., O. Avidan, and A. Hizi. 1997. The fidelity of misinsertion and mispair extension throughout DNA synthesis exhibited by mutants of the reverse transcriptase of human immunodeficiency virus type 2 resistant to nucleoside analogs. Eur. J. Biochem. 250:106-114.

98. Temesgen, Z., and A. J. Wright. 1999. Antiretrovirals. Mayo. Clin. Proc. 74:1284-1301.

99. Temin, H. M. 1976. The DNA provirus hypothesis. Science 192:1075-1080.

100. Temin, H. M. 1993. Retrovirus variation and reverse transcription: abnormal strand transfers result in retrovirus genetic variation. Proc. Natl. Acad. Sci. USA 90:6900-6903.

101. Thomas, M. J., A. A. Platas, and D. K. Hawley. 1998. Transcriptional fidelity and proofreading by RNA polymerase II. Cell 93:627-637.

102. Tisdale, M., S. D. Kemp, N. R. Parry, and B. A. Larder. 1993. Rapid in vitro selection of human immunodeficiency virus type 1 resistant to 3 -thiacytidine inhibitors due to a mutation in the YMDD region of reverse transcriptase. Proc. Natl. Acad. Sci. USA 90:5653-5656.

103. van der Groen, G., P. N. Nyambi, E. Beirnaert, D. Davis, K. Fransen, L. Heyndrickx, P. Ondoa, G. Van der Auwera, and W. Janssens. 1998. Genetic 
variation of HIV type 1: relevance of interclade variation to vaccine development. AIDS Res. Hum. Retroviruses 14 Suppl 3:S211-221.

104. Vartanian, J. P., A. Meyerhans, M. Sala, and S. Wain-Hobson. 1994. G-->A hypermutation of the human immunodeficiency virus type 1 genome: evidence for dCTP pool imbalance during reverse transcription. Proc. Natl. Acad. Sci. USA 91:3092-3096.

105. Wainberg, M. A., W. C. Drosopoulos, H. Salomon, M. Hsu, G. Borkow, M. Parniak, Z. Gu, Q. Song, J. Manne, S. Islam, G. Castriota, and V. R. Prasad. 1996. Enhanced fidelity of 3TC-selected mutant HIV-1 reverse transcriptase. Science 271:1282-1285.

106. Wakefield, J. K., S. A. Jablonski, and C. D. Morrow. 1992. In vitro enzymatic activity of human immunodeficiency virus type 1 reverse transcriptase mutants in the highly conserved YMDD amino acid motif correlates with the infectious potential of the proviral genome. J. Virol. 66:6806-6812.

107. Wisniewski, M., C. Palaniappan, Z. Fu, S. F. Le Grice, P. Fay, and R. A. Bambara. 1999. Mutations in the primer grip region of HIV reverse transcriptase can increase replication fidelity. J. Biol. Chem. 274:28175-28184. 


\section{CHAPTER 2}

\section{Development of an In Vivo Assay to Identify Structural Determinants in Murine Leukemia Virus Reverse Transcriptase Important for Fidelity}

Elias K. Halvas, Evguenia S. Svarovskaia, and Vinay K. Pathak*

Mary Babb Randolph Cancer Center and Department of Biochemistry, West Virginia University, Morgantown, WV 26506.

Running Title: Fidelity Determinants of MLV Reverse Transcriptase

This manuscript was published in the January 2000 issue of Journal of Virology

(Vol. 74, No. 1, p. 312-319) 


\begin{abstract}
Error-prone DNA synthesis by retroviral reverse transcriptases (RTs) is a major contributor to variation in retroviral populations. Structural features of retroviral RTs that are important for accuracy of DNA synthesis in vivo are currently unknown. To identify structural elements of murine leukemia virus (MLV) RT important for fidelity in vivo, we developed a D17-based encapsidating cell line (ANGIE P) which is designed to express the amphotropic MLV envelope. ANGIE $P$ also contains an MLV-based retroviral vector (GA-1), which encodes a wildtype bacterial $\beta$-galactosidase gene (lacZ) and a neomycin phosphotransferase gene. Transfection of ANGIE $P$ cells with wildtype or mutated MLV gag-pol expression constructs generated GA-1 virus that was able to undergo only one cycle of viral replication upon infection of D17 cells. The infected D17 cell clones were characterized by staining with X-Gal and the frequencies of inactivating mutations in $\operatorname{lac} Z$ were quantified. Three mutations in the YVDD motif (V223M, V223S, and V223A) and two mutations in the RNase H domain (S526A and R657S) exhibited 1.2- to 2.3-fold higher frequencies of $l a c Z$ inactivation in comparison to the wildtype MLV RT $(P<$ 0.005). Two mutations (V223I and Y598V) did not affect the frequency of lacZ inactivation. These results establish a sensitive in vivo assay for identification of structural determinants important for accuracy of DNA synthesis and indicate that several structural determinants may have an effect on the in vivo fidelity of MLV RT.
\end{abstract}




\section{INTRODUCTION}

High mutation rates exhibited by retroviruses generate extensive genetic variation and confer high evolutionary potential in retroviral populations $(14,42,43,52-54,62)$. Variation in human immunodeficiency virus type 1 (HIV-1) populations may in turn affect the fitness of the species. For example, genetic variation in HIV-1 populations has resulted in the selection of mutations that confer resistance to at least 11 antiretroviral drugs approved for clinical use and selection of escape mutants that evade the host immune response $(15,46)$. Genetic variation has also thwarted efforts to develop effective vaccines against HIV-1(64).

An important mechanism of generating variation in retroviral populations is the low fidelity of reverse transcriptase (RT) $(42,43,52-54)$. Mutation rates of RTs can be measured in vitro or in vivo by employing the use of various assays $(5,42,43,53-55)$. The measurements of fidelity are often altered by the in vitro assay conditions $(18,39,44)$. Previously, the in vitro mutation rate of HIV-1 was estimated to be approximately 20 -fold higher than the in vivo mutation rate $(4,42)$. In vivo forward mutation assays for various RTs have been determined and range from $1.2 \times 10^{-5}$ to $4.8 \times 10^{-6}$ mutations/bp/replication cycle $(42,43,52-54)$.

Amino acid positions and motifs in RT that may be important for the in vivo fidelity of RT are currently unknown. However, several studies have identified potential structural determinants that are important for the in vitro fidelity of RT $(1-3,19,25-27$, $35,37,45,50,51,56,57,60,66)$. The YXDD motif is highly conserved in RTs and mutations in the motif are associated with decreases in enzymatic activity and viral 
infectivity, changes in the positioning of the primer in the template-primer complex, and resistance to nucleoside analogs $(10,12,15,59,67)$. In addition, mutations at the methionine of the HIV-1 YMDD motif have been associated with alterations in the in vitro fidelity of RT $(1,2,19,25-27,50,51,66)$. Mutation of the HIV-1 YMDD motif to YVDD (M184V) is associated with high level resistance to the antiretroviral drug (-) 2', 3' dideoxy-3'-thiacytidine (3TC) (9, 20, 63). Several studies have observed large increases (up to 45-fold) in the in vitro fidelity of HIV-1 RT containing the M184V mutation $(50,51,66)$. However, another in vitro study using a forward mutation assay has indicated that the YVDD mutant of HIV-1 RT exhibits less than a 2-fold decrease in the mutation rate (19).

The amino acids involved in binding to the substrate dNTP may also constitute a fidelity determinant of RT $(26,29)$. Recently, the crystal structure of HIV-1 RT complexed with the template-primer and the dTTP substrate shows six amino acids that contact the substrate dTTP (29). Mutations at three of the positions were associated with alterations in the in vitro fidelity of $\mathrm{RT}(26,45)$. Another motif, the $\alpha \mathrm{H}$ helix of the HIV1 RT thumb domain, binds to the minor groove of the template-primer complex and is associated with alterations in the processivity and fidelity of RT $(3,6,30)$. Mutational analysis of the $\alpha \mathrm{H}$ helix of HIV-1 RT was associated with lower fidelity in vitro, either through an increase in the rate of RT dissociation from the template DNA or through an increase in strand slippage resulting in lower frameshift fidelity (6). Finally, the RNase H domain may influence the rate of RT processivity and template switching, which may result in an increase in the rate of deletions, deletions with insertions, and duplications (21, $24,33,41)$. 
The differences in the fidelities of HIV-1 RT mutants in different in vitro assays demonstrate a need for analyzing the fidelity of RTs during the course of in vivo retroviral replication. Structural similarities between MLV and HIV-1 RTs suggests that understanding the structural determinants of MLV RT important for fidelity may provide insights into the fidelity of HIV-1 RT $(22,38)$. In addition, a greater understanding of the mechanisms of MLV RT fidelity may lead to the development of improved MLV-based vectors and packaging cell lines for gene therapy. In this study, we have developed an in vivo assay for rapid measurement of the fidelity of wild type or mutated MLV RTs. This assay provides a useful tool to identify structural determinants of MLV RT important for accuracy of DNA synthesis and to determine the extent to which they influence fidelity in vivo.

\section{MATERIALS AND METHODS}

Plasmids and retroviral vector. The construction of MLV-based retroviral vector GA-1 was previously described (34). The plasmids pLGPS and pAMS, which expressed the MLV gag and pol genes from a truncated MLV LTR promoter or expressed a replication competent MLV, respectively, were a kind gift from A. D. Miller (47, 48). The plasmid pSV-A-MLV-env, which expressed the amphotropic MLV envelope gene from the LTR promoter and SV40 enhancer, was obtained from the AIDS Research and Reference Reagents Program (40). The plasmid pBSpac encoded the puromycin $\mathrm{N}$-acetyl transferase gene and conferred resistance to puromycin (65). The plasmid pSV $\alpha 3.6$ encodes the $\alpha$ subunit of murine $\mathrm{Na}^{+}, \mathrm{K}^{+}$-ATPase gene and confers resistance to ouabain (36). 
Generation of MLV RT mutants. Site-directed mutagenesis of pLGPS was performed by utilizing the Morph Kit (5'-3', Inc.) or the Chameleon kit (Stratagene). A detailed description of the mutagenic oligonucleotides and the strategies used to identify mutants is available upon request. Briefly, most of the mutagenic oligonucleotides were designed to introduce additional silent mutations and generate new restriction sites. Restriction digestion analysis was performed to identify plasmids containing mutations. DNA fragments ranging in size from 479-1230 bp from the plasmids containing mutations were subcloned into pLGPS. The inserted fragments were analyzed by DNA sequencing to verify the presence of the desired mutation and the absence of any undesired mutations (ALF Automated Sequencer, Pharmacia).

Cells, transfections, and infections. D17 dog osteosarcoma cells were transfected, infected with MLV, and selected for resistance to ouabain or G418 (a neomycin analog) as previously described $(33,34)$. The D17 cells were selected for resistance to puromycin (final concentration, $3.2 \times 10^{-6} \mathrm{M}$ ). The D17-derived ANGIE P cells were maintained, transfected, and selected for resistance to ouabain in a similar manner.

Protocol for determination of in vivo fidelity. The ANGIE P cells were plated at a density of $2 \times 10^{5}$ cells per 60 -mm diameter dish, and $24 \mathrm{~h}$ later cotransfected with wildtype or mutated pLGPS and pSV 23.6 . The transfected cells were selected for resistance to ouabain and the resistant colonies were pooled and expanded. The ouabainresistant cells were plated at a density of $5 \times 10^{6}$ cells per $100-\mathrm{mm}$ diameter dish; $48 \mathrm{~h}$ later the culture media containing GA-1 virus was harvested and used to infect D17 target cells. The infected D17 cells that were resistant to G418 were stained with X-Gal and the 
frequency of lac $Z$ inactivation was determined by quantification of lac $Z$ expressing blue colonies and non-expressing white colonies.

Virus preparation and reverse transcriptase assay. To isolate and concentrate virus, helper cells containing different constructs were plated out at $5 \times 10^{6}$ cells per 100 $\mathrm{mm}$ dish. Viruses were collected 2 days later and centrifuged at 25,000 rpm for $90 \mathrm{~min}$ in an SW41 rotor (Beckman) at $4^{0} \mathrm{C}$. Viral pellets were resuspended in culture media or phosphate buffered saline (PBS) and virus was stored at $-80^{\circ} \mathrm{C}$. Concentrated virus was thawed on ice and the reverse transcriptase activities were determined by modification of previously described method (23). Briefly, $50 \mu \mathrm{g} / \mathrm{ml}$ of (20-mer) oligo T (Integrated DNA Technologies), $100 \mu \mathrm{g} / \mathrm{ml}$ poly A (Pharmacia), and $10 \mu \mathrm{Ci}{ }^{3} \mathrm{H}$ dTTP (specific activity of $72 \mathrm{Ci} / \mathrm{mMol}, \mathrm{ICN}$ ) were used in the reactions. The reaction mixtures were precipitated with $10 \%$ trichloroacetic acid (Sigma), filtered through $0.45 \mu \mathrm{m}$ Metricel membranes (Gelman Sciences, Inc.), and the incorporated ${ }^{3} \mathrm{H}$ dTTP was determined using a scintillation counter.

Western blot analysis. Western blots used to quantitate the amount of protein for the RT assay were performed using standard procedures (58). Briefly, viral proteins were resolved on a 15\% SDS-polyacrylamide gel, transferred to membranes (Gelman Sciences, Inc.), and incubated with a 1:10 dilution of a rat anti-MLV capsid monoclonal antibody (13). The membranes were further incubated with anti-rat IgG antibody conjugated with horseradish peroxidase (Southern Biotechnology Associates, Inc.) at a 1:10,000 dilution. The MLV capsid was detected using an ECL kit ${ }^{\text {TM }}$ (Amersham Pharmacia Biotech) and quantitative autoradiography using a densitometric program (Optimas). 
PCR analysis and characterization of proviral DNA. A total of 161 individual G418-resistant clones infected with GA-1 were isolated and grown in duplicate 24-well tissue culture dishes. Mutant colonies were identified by staining one $24 \mathrm{~mm}$-well dish with X-Gal. Eleven mutant cell clones and six wildtype cell clones were expanded on 60mm diameter dishes. The expanded cells were lysed and proviral DNAs amplified by two rounds of PCR $(28,58)$.

Three pairs of forward and reverse primers that annealed to six different regions in GA-1 were employed to amplify three overlapping fragments of lacZ. DNAs obtained from the first round of PCR were further amplified by a second round of PCR utilizing nested primer sets (58). PCR products (1216-1326 bp) were further analyzed for smaller deletions or insertions by digestions with various restriction enzymes (BstUI, HaeIII, HhaI, DpnII, HpaII, and NlaIII) and electrophoresis using 7\% polyacrylamide gels or 3\% MetaPhor gels (MetaPhor agarose, FMC).

Southern analysis of proviral DNA. Genomic DNA was isolated from the ANGIE P ecapsidating cell line and the proviral structure was analyzed by Southern blot hybridization using standard procedures $(16,58)$. A 1.2-kb fragment of WH390 containing IRES-neo was used to generate a probe (specific activity $>10^{9} \mathrm{cpm} / \mu \mathrm{g}$ ) as previously described (16). Quantitation of bands was accomplished with the ImageQuant program (Molecular Dynamics).

\section{RESULTS}

Construction of ANGIE $\mathbf{P}$ encapsidating cell line. The MLV-based retroviral vector pGA-1 and the MLV envelope expression construct pSV-A-MLV-env (Fig. 1A) were introduced into D17 cells to generate the ANGIE P encapsidating cell line (Fig. 1B). 
The vector GA-1 possesses all cis-acting elements needed for viral replication, which include the long terminal repeats (LTRs), the MLV encapsidation signal $(\Psi)$, primer binding site (PBS), and the polypurine tract (PPT). In addition, the vector expresses the bacterial $\beta$-galactosidase gene (lacZ) and the neomycin phosphotransferase gene (nеo) from a single RNA transcript initiating in the 5' LTR. An internal ribosomal entry site (IRES) of encephalomyocarditis virus is used to express neo (31). The construct pSV-AMLV-env expresses the amphotropic MLV envelope using a modified MLV LTR promoter.

To construct the ANGIE P encapsidating cell line, pGA-1 was first transfected into the MLV-based packaging cell line PG13 and G418 resistant cells were selected. Virus harvested from the pool of transfected PG13 cells was used to infect D17 cells. After selection for G418 resistance, individual D17 cell clones were isolated and expanded. To insure that the provirus in the D17 cell clones expressed a functional lacZ, six cell clones were stained with X-Gal and clones that stained blue were selected for further manipulation (data not shown). To verify that the GA-1 provirus was capable of completing one cycle of retroviral replication, the D17 cell clones were transfected with pAMS, a plasmid that encodes a replication competent MLV. After transfection, the replication competent virus was allowed to spread through the culture for 5 to 7 days. Virus was harvested and used to transform fresh D17 cells. The presence of cells that stained blue with X-Gal after infection indicated that the GA-1 provirus was mobilized from the D17 cell clones to the target cells in the presence of the MLV helper virus (data not shown). 
Next, the pSV-A-MLV env was cotransfected with pBSpac (a plasmid that confers resistance to puromycin) into a D17 cell clone containing a GA-1 provirus. The transfected cells were selected for resistance to puromycin and 12 cell clones were isolated and expanded. To verify that the cell clones expressed a functional amphotropic MLV envelope, the cell clones were cotransfected with pLGPS, which expresses the MLV GagPol proteins (Fig. 1A) and pSV 3.6 (a plasmid that confers resistance to ouabain). Ouabain resistant cells were selected and virus produced from them was used to infect fresh D17 cells. The infected D17 cells were selected for resistance to G418 and stained with X-Gal (11). The presence of cells that stained blue with X-Gal indicated that the D17 cell clones expressed the amphotropic MLV envelope and mobilized the GA-1 provirus to target cells in the presence of MLV Gag-Pol proteins. All 12 of the D17 cell clones tested for expression of the amphotropic MLV envelope generated virus titers of at least $10^{4} \mathrm{CFU} / \mathrm{ml}$ after transfection with pLGPS (data not shown). One of the 12 clones was chosen and named the ANGIE P encapsidating cell line (Fig. 1B).

It was also important to show that the ANGIE P encapsidating cell line did not express a replication competent MLV, the presence of which could potentially result in multiple rounds of GA-1 replication. Culture supernatant from the ANGIE P encapsidating cell line was used to infect fresh D17 cells, which were selected for resistance to G418. No G418-resistant colonies were observed, indicating that the ANGIE P encapsidating cell line did not harbor a replication competent virus (data not shown). Finally, the ANGIE P cells were plated at a low density ( $<100$ cell/60-mm dish) and stained with X-Gal. The frequency of white cells was approximately 1 per 1500 cells, 
indicating that the vast majority of the virus is produced from cells containing a functional lacZ (data not shown).

To ensure that the ANGIE P encapsidating cell line contained only one GA-1 provirus, genomic DNA was extracted from the ANGIE P cells and analyzed by Southern blot hybridization (Fig. 1C). The DNA was digested with either HindIII or NcoI and hybridized to a probe containing IRES and neo sequences (shown in Fig. 1A). The HindIII enzyme cuts once in the GA-1 provirus and two fragments of varying lengths, representing $5^{\prime}$ and $3^{\prime}$ portions of the provirus and flanking regions, should be generated from each provirus. The presence of only two detectable fragments after HindIII digestion indicated that only one provirus was present in the ANGIE P cells. Similarly, the NcoI enzyme cuts once in the GA-1 provirus and one fragment representing the 5' portion of the provirus and flanking region should be generated from each provirus. The presence of one fragment after $\mathrm{NcoI}$ digestion verified that only one provirus was present in the ANGIE P cells (Fig. 1C). Finally, the sequence of the lac Z in ANGIE P cells was determined by PCR amplification and DNA sequencing of both strands to insure that no mutations were present (data not shown).

Experimental protocol. The MLV RT expression construct pLGPS was subjected to site directed mutagenesis, and the ANGIE P cells were employed to identify protein determinants important to the accuracy of DNA synthesis during reverse transcription (Fig. 1A and B). First, pLGPS-derived constructs that contained mutations in the MLV RT were separately introduced into the ANGIE P cells by cotransfection with pSV 3.6. Ouabain-resistant colonies were pooled and expanded. Virus was harvested from the pools of transfected cells, serially diluted viruses were used to infect D17 target 
cells. The infected D17 cells were selected for resistance to G418 and the resulting colonies were stained with $\mathrm{X}-\mathrm{Gal}$. The frequency of lacZ inactivation was determined by dividing the number of white colonies by the total number of colonies (blue plus white colonies). In general, the multiplicity of infection was $<0.0005$, and the probability of double infection was very low $(<1 / 2000$ colonies). The frequency of lacZ inactivation provided a measure of the inactivating mutations introduced into the lac $Z$ gene during one cycle of retroviral replication, which constituted the transfer of genetic information from the GA-1 provirus in the ANGIE P cells to the GA-1 provirus in the D17 target cells. Using this assay, the effect of introducing mutations in the MLV RT on the fidelity of DNA synthesis was determined. In addition, the virus titers obtained after transfections of wildtype or mutated pLGPS constructs were used to determine the effect of mutations on the efficiency of virus replication.

\section{Effect of mutations at the V223 position of the MLV YVDD motif on fidelity.}

The V223 of MLV RT was mutated to an alanine (V223A), isoleucine (V223I), methionine $(\mathrm{V} 223 \mathrm{M})$, or serine $(\mathrm{V} 223 \mathrm{~S})$ and the effect of these mutations on the frequency of lac $Z$ inactivation was determined. The frequencies of $l a c Z$ inactivation with each mutant RT were compared to the frequency of inactivation obtained with wildtype RT in parallel experiments (Table 1).

The wildtype pLGPS construct inactivated lac $Z$ at an average frequency of 5.2\% in one cycle of retroviral replication. The frequencies of lacZ inactivation obtained in 10 independent experiments were highly reproducible (S. E. \pm 0.48\%). The V223A, V223M, and V223S mutants exhibited a 1.7-, 1.8-, and 2.3-fold higher frequencies of lacZ inactivation, respectively, in comparison to wildtype. The observed frequencies of lac $Z$ 
inactivation with the mutants were shown to be statistically different than wildtype ( $P<$ 0.001). Conversely, the isoleucine mutant showed no significant change in the frequency of lacZ inactivation $(5.3 \% \pm 0.30)$. All mutants displayed decreases in viral titers relative to the wildtype $\left(4.4 \times 10^{4} \mathrm{CFU} / \mathrm{ml}\right)$, which ranged from 2- to 100-fold (Table 1 and Fig. 2C). In addition, there was no correlation between reduction in viral titer and fidelity.

Mutational analysis of MLV RT RNase H domain. Mutations S526A, Y598V, and R657S were introduced into the MLV RNase H domain, and the effects of these mutations on the frequency of lac $Z$ inactivation were determined (Table 2). These mutations were selected because it was previously shown that they did not affect the polymerase activity and permitted viral replication to occur $(7,8)$. Again, the wildtype RT displayed a $5.2 \% \pm 0.30$ frequency of lacZ inactivation. Mutants R657S and S526A exhibited frequencies of lacZ inactivation that were increased by 1.2- and 1.4-fold, respectively $(P<0.005)$. In contrast, the Y598V mutant did not exhibit a change in the frequency of lacZ inactivation $(5.4 \% \pm 0.39 ; P=0.81)$. Finally, all three RNase $\mathrm{H}$ domain mutants exhibited a decrease in the viral titer by 2.5 - or 5 -fold relative to the wildtype ( 1.2 $\times 10^{5} \mathrm{CFU} / \mathrm{ml}$ ) (Table 2 and Fig. 2C). Once again, there was no correlation between reduction in viral titer and fidelity.

Reverse transcriptase activities of mutant RTs. Viruses produced from the wildtype and mutant RTs were harvested by ultracentrifugation and characterized by Western blotting analysis using an anti-MLV capsid antibody (13). The Western blots were quantified to estimate the amounts of MLV capsid protein present in the viral preparations. A representative blot is shown in Fig. 2A. The estimated amounts of capsid 
protein were utilized to insure that equivalent amounts of viral proteins were used for determination of reverse transcriptase activities (Fig. 2B). The reverse transcriptase activities ranged from $5 \% \pm 1 \%$ for the V223A mutant to $93 \% \pm 1 \%$ for the S526A mutant, relative to the wildtype enzyme. As expected, all of the RNase $\mathrm{H}$ domain mutants exhibited RT activities that were comparable to the wildtype RT. With the exception of the V223I mutant, all of the reductions in the reverse transcriptase activities were significantly different from the wildtype $(P<0.005)$.

The reverse transcriptase activities were compared to the relative reductions in viral titers observed (Fig. 2C). In general, the reductions in reverse transcriptase activities were proportional to the reductions in viral titers observed for the V223 mutants. As expected, the RNase $\mathrm{H}$ mutants exhibited higher reverse transcriptase activities than most of the V223 mutants at the polymerase active site. In general, the RNase $\mathrm{H}$ mutants displayed a greater reduction in the viral titer relative to the reverse transcriptase activities, suggesting that other steps in the reverse transcription process were affected by mutations introduced in the RNase $\mathrm{H}$ domain.

Analysis of lacZ inactivating mutations. To determine the nature of mutations introduced into the lacZ gene by the wildtype or mutant RTs, 161 infected G418-resistant cell clones were isolated from infections performed with the wildtype RT as well as the V223A, V223M, and the Y598V mutants. X-Gal staining of the clones indicated that 11 of 161 cell clones exhibited a white colony phenotype. One mutant clone was derived from infection with the wildtype RT, three were derived from infection with the V223A mutant, three were derived from infection with the Y598V mutant, and four were derived from infection with the V223M mutant. The lac $Z$ sequences from these 11 white colonies 
was amplified by two rounds of PCR using six different sets of primers. As a result, 3 overlapping fragments designated the 5', the middle, and the 3' portions were generated (Fig. 3A). The PCR products were approximately 1.2 to $1.3 \mathrm{~kb}$ in length. Each of the PCR fragments derived from the 11 cell clones were analyzed by gel electrophoresis and compared to the fragments obtained from a cell clone containing a functional lacZ. This analysis indicated that none of the lac $Z$ genes present in the 11 cell clones were inactivated by large deletions or insertions (> $100 \mathrm{bp}$; data not shown). The amplified lacZ fragments were digested with various restriction enzymes to generate smaller fragments in an effort to identify shorter deletions or insertions (Fig. 3B). Both the 5' and 3' fragments of the lacZ from infected cell clones were digested with Bst UI, HaeIII, and HhaI. The middle portion was digested with DpnII, HpaII, and NlaIII. DNA fragments that ranged in size from 75 to 863 bp were analyzed by gel electrophoresis. No differences were observed in any of the restriction digestions, indicating that the $l a c Z$ inactivating mutations did not involve deletions or insertions that were larger than $20 \mathrm{bp}$ (based on the estimated resolution the restriction analysis).

Since no small deletions or insertions could be observed, both strands of the PCR products derived from 3 cell clones were sequenced to insure the accuracy of the sequencing analysis. These three cell clones that displayed a white colony phenotype were derived from an infection with the V223A mutant of MLV RT. The sequencing analysis indicated that two mutants contained a +1 frameshift in runs of 6 adenosine nucleotides present at different locations in the lac $Z$ gene. One mutant contained a $\mathrm{C}$ to $\mathrm{T}$ transition, which resulted in the generation of a termination codon. These mutations were not the result of errors occurring during the PCR reactions, since they were identified in 
independent PCR reactions (data not shown). These results verified that the white colony phenotype was indeed generated by introduction of mutations in lacZ. In addition, different types of mutations could be identified in this assay (Fig. 3C).

\section{DISCUSSION}

This study provides a rapid, accurate, and highly reproducible in vivo assay for identification of MLV RT structural determinants important for fidelity. The in vivo assay described here is rapid in comparison to the previously described in vivo assays involving shuttle vectors because the mutant frequencies can be determined immediately after the infected cell clones are obtained $(42,43,53,54)$. This assay is also more sensitive and accurate than the previously described in vivo assay utilizing the herpes virus thymidine kinase reporter gene because it is not necessary to compare viral titers after two different drug selection procedures (52). Due to the large size of the lac $Z$ reporter gene, a high mutant frequency is obtained and a large number of mutants can be identified. The high mutant frequency increases the utility of the assay because the accuracy of RT mutants with low viral titers can be easily determined. In addition, mutations that increase the accuracy of DNA synthesis can also be identified. One limitation of this in vivo assay is that RT mutants with extremely low fidelities may generate highly mutated viruses that can not confer resistance to G418 and therefore can not be analyzed.

The results of these studies indicate that mutations at the V223 position of the MLV RT have a statistically significant effect on the in vivo fidelity of RT. These results are consistent with the in vitro forward mutation assays performed with the YVDD mutant of HIV-1 RT (19). For both MLV and HIV-1 RTs, the YVDD motif appears to be 
approximately twofold more accurate than the YMDD motif. It is possible that even though the overall mutation rate is altered by only twofold, the rate of a specific type of mutation is altered to a greater extent. For example, a 10 -fold increase in the rate of one of the twelve possible substitutions may have a less than twofold effect on the overall mutation rate.

The effect of a twofold alteration in the retroviral mutation rate on variation in viral populations is undetermined. A model was previously proposed to ascertain the relative impact of mutation rates and selective forces on viral populations (14). Based on the model, it was hypothesized that in a large viral population with a high replicative capacity, small changes in the selective forces will have a greater impact on the viral variants present than large changes in mutation rates. Whether small changes in the mutation rate will affect viral evolution will be dependent on the size of the viral population and its growth potential. In this regard, it is significant that the rate of development of drug resistant variants in cell culture was the same for the YMDD and the YVDD variants of HIV-1 $(32,49)$. This observation, along with the observation that the YVDD variant is only twofold more accurate than the YMDD variant, suggests that twofold alterations in RT fidelity are unlikely to have an effect on the rate of viral evolution.

It is of interest to consider the structural features of the YMDD and YVDD variants and how these features may be related to the statistically significant alterations in fidelity. It has been postulated that the X residue of the YXDD motif may contact the primer and is important for affinity to the template-primer as well as its positioning relative to the catalytic site $(10,17,59)$. Repositioning of the template-primer by the YMDD 
variant may alter flexibility of the dNTP binding pocket, ultimately leading to changes in fidelity (27). In addition, molecular modeling studies have predicted an increased fidelity of YVDD and YIDD variants due to new contacts with the incoming dNTP substrate that are absent from the YMDD variant $(29,51)$. Therefore, the hydrophobicity and the size of the amino acid side chain at the V223 position may be important for the catalytic function and fidelity of RT. It is interesting to note that 2 of the 3 mutations generated with the V223A mutant RT were +1 frameshifts. Frameshifts account for approximately $10 \%$ of all mutations $(42,54)$, and this limited analysis suggests that the V223A mutation may affect the template-primer affinity and frameshift fidelity, which is consistent with previous observations (10).

The observation that the V223A mutant exhibits a severe reduction in the polymerase activity whereas the V223I mutant has little effect on the polymerase activity is consistent with previously published results obtained with HIV-1 RT (10). Also, the viral titers were consistently reduced to a greater extent than the observed decreases in polymerase activities, suggesting that additional steps during reverse transcription may also be affected by the mutations.

The RNase $\mathrm{H}$ mutants S526A and R657S exhibited small but statistically significant alterations in fidelity. As expected, the mutations had little effect on the polymerase activity (8). The changes in fidelity of these mutants may be due to altered processivity of RT, interactions of the RT with the template-primer complex, or the overall structure of the RT $(24,61)$. The reductions in viral titers associated with these mutants may be due to a reduced efficiency of strand transfer events during reverse transcription $(21,41)$. 
The assay described here may be utilized to determine the role of various RT domains, including the dNTP binding site and the thumb domain, in the fidelity of reverse transcription. The importance of other viral proteins to the fidelity of reverse transcription may also be easily determined. Finally, a similar strategy may be utilized to analyze the effect of mutations in RT on the frequencies of specific types of mutations.

\section{ACKNOWLEDGEMENTS}

We thank Jeffrey Anderson, Benjamin Beasley, Sara Cheslock, Que Dang, Krista Delviks, Wei-Shau Hu, Carey Hwang, Timor Kubdalov, Yegor Voronin and Wei-hui Zhang for critical reading of the manuscript and discussion of results. We especially thank Wei-Shau Hu for her valuable intellectual input and discussions throughout this project.

This work was supported by the Public Health Service grant CA58875 for the National Institutes of Health. 
TABLE 1. The effects of V223 mutations in the YVDD motif of MLV RT on the frequency of $l a c Z$ inactivation

\begin{tabular}{|c|c|c|c|c|c|}
\hline $\begin{array}{l}\text { MLV-RT } \\
\text { Genotype }\end{array}$ & $\begin{array}{l}\text { No. of } \\
\text { Expts }\end{array}$ & $\begin{array}{c}\text { No. Mut. } \\
\text { Colonies/Total } \\
\text { Colonies }^{\mathrm{a}}\end{array}$ & $\begin{array}{l}\text { Frequency } \\
\text { of lacZ } \\
\text { Inactivation } \\
(\% \pm \text { S.E. })^{\mathrm{b}}\end{array}$ & $\begin{array}{l}\text { Relative } \\
\text { Change in } \\
\text { Inactivation } \\
\text { of } l a c Z^{c}\end{array}$ & $\begin{array}{c}\text { Relative } \\
\text { Viral Titer }^{\mathrm{d}}\end{array}$ \\
\hline $\mathrm{Wt}$ & 10 & $154 / 2,860$ & $5.2 \pm 0.48$ & 1.0 & 1.0 \\
\hline V223A & 6 & $207 / 2,289$ & $8.8 \pm 0.45$ & 1.7 & 0.03 \\
\hline V223I & 2 & $95 / 1,764$ & $5.3 \pm 0.30$ & 1.0 & 0.50 \\
\hline V223M & 4 & $182 / 1,974$ & $9.2 \pm 0.36$ & 1.8 & 0.12 \\
\hline V223S & 4 & $200 / 1,632$ & $12.1 \pm 0.43$ & 2.3 & 0.01 \\
\hline
\end{tabular}

${ }^{\mathrm{a}}$ The number of mutant colonies that displayed a white colony phenotype and the total number of colonies that were observed in 2 to 10 independent experiments.

${ }^{\mathrm{b}}$ The frequency of lacZ inactivation was calculated as follows: No. mutant colonies in each experiment $\div$ by total number of colonies $\times 100 \%$. The standard errors were determined by using the Two-Sample T-Test.

c The relative change in the frequency at which the lac $Z$ gene was inactivated was calculated as follows: frequency of lacZ inactivation observed with mutant MLV RT $\div$ by frequency of lacZ inactivation observed with wildtype MLV RT. Statistical analysis using a Two-sample T-Test showed that V223M, V223S, and YADD mutants of MLV RT displayed a significantly higher mutant frequency in comparison to the wildtype MLV RT $(P<0.001)$. The mutant frequency obtained with the YIDD mutant of MLV RT was not significantly different from the wildtype $\operatorname{MLV}$ RT $(P=0.71)$.

${ }^{\mathrm{d}}$ Virus titer for each experimental group was determined by serial dilutions and infections. The average virus titer obtained with wildtype MLV RT was 4.4 x $10^{4} \mathrm{CFU} / \mathrm{ml}$. The relative virus titer represents a ratio of the virus titer of mutant MLV RT obtained in each 
experiment divided by the virus titer obtained with wildtype MLV RT in parallel experiments. 
TABLE 2. The effects of mutations in the RNase H domain of MLV RT on the frequency of lac Z inactivation

\begin{tabular}{|c|c|c|c|c|c|}
\hline $\begin{array}{l}\text { MLV-RT } \\
\text { Genotype }\end{array}$ & $\begin{array}{l}\text { No. of } \\
\text { Expts }\end{array}$ & $\begin{array}{l}\text { No. Mut. } \\
\text { Colonies/Total } \\
\text { Colonies }^{\mathrm{a}}\end{array}$ & $\begin{array}{l}\text { Frequency } \\
\text { of lacZ } \\
\text { Inactivation } \\
(\% \pm \text { S.E. })^{b}\end{array}$ & $\begin{array}{l}\text { Relative } \\
\text { Change in } \\
\text { Inactivation } \\
\text { of } l a c Z^{c}\end{array}$ & $\begin{array}{c}\text { Relative } \\
\text { Viral Titer }^{\mathrm{d}}\end{array}$ \\
\hline $\mathrm{Wt}$ & 9 & $473 / 8,023$ & $5.2 \pm 0.30$ & 1 & 1.0 \\
\hline S526A & 5 & $429 / 5,039$ & $8.4 \pm 0.33$ & 1.6 & 0.25 \\
\hline Y598V & 4 & $181 / 3,534$ & $5.4 \pm 0.39$ & 1 & 0.40 \\
\hline R657S & 5 & $344 / 4,754$ & $7.1 \pm 0.14$ & 1.4 & 0.21 \\
\hline
\end{tabular}

${ }^{\mathrm{a}}$ The number of mutant colonies that displayed a white colony phenotype and the total number of colonies that were observed in 4 to 9 independent experiments.

${ }^{\mathrm{b}}$ The frequency of lac $Z$ inactivation was calculated according to Table 1 .

${ }^{c}$ The relative change in the frequency at which the lac $Z$ gene was inactivated was calculated at described in Table 1. Statistical analysis using a Two-Sample T-Test showed that S526A and R657S mutants of MLV RT displayed a significantly higher mutant frequency in comparison to the wildtype MLV RT $(P<0.005)$. The mutant frequency obtained with the Y598V mutant of MLV RT was not significantly different from the wildtype MLV RT $(P=0.81)$.

${ }^{\mathrm{d}}$ Virus titer for each experimental group was determined by serial dilutions and infections. The average virus titer obtained with wildtype MLV RT was $1.2 \times 10^{5} \mathrm{CFU} / \mathrm{ml}$. The relative virus titer represents a ratio of the virus titer of mutant MLV RT obtained in each experiment divided by the virus titer obtained with wildtype MLV RT in parallel experiments. 
FIG. 1. Structures of MLV-based constructs and rapid in vivo assay to identify structural determinants in MLV RT important for fidelity. (A) Structures of MLV-based vector pGA-1, pLGPS, and pSV-A-MLV-env. The pGA-1 vector contains long terminal repeats (LTRs) and all cis-acting elements of MLV. pGA-1 transcribes the E. coli lacZ and neo from the LTR promoter. The internal ribosomal entry site (IRES) of encephalomyocardidtis virus is used to express neo. The construct pLGPS expresses the MLV gag and pol from a truncated viral LTR. Finally, the pSV-A-MLV-env expresses the amphotropic MLV envelope from a truncated MLV LTR and the SV40 promoter enhancer. (B) Experimental protocol. ANGIE P, a D17-based cell line expressing pGA-1 and amphotropic MLV envelope, was constructed. The wildtype or mutated pLGPS were separately cotransfected with $\mathrm{pSV} \alpha 3.6$ into the ANGIE P cell line and the virus produced was used to infect D17 cells. The infected cell clones resistant to G418 were stained with $\mathrm{X}-\mathrm{Gal}$ and the numbers of blue (wildtype) and white (mutant) colonies were determined. (C) Southern analysis of the ANGIE P encapsidating cell line. Genomic DNA isolated from ANGIE P cells was digested with either HindIII (lane H) or NcoI (lane N). The blots were probed with IRES-neo (black bar, Fig. 1A) and quantitated by PhosphorImager analysis. 
A.

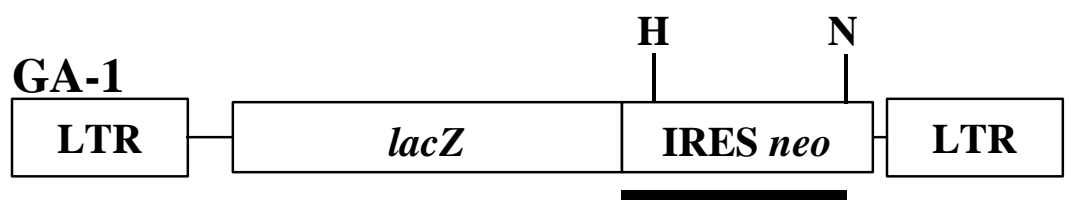

\section{pLGPS}

\begin{tabular}{|c|c|c|c|}
\hline$\Delta \mathrm{LTR}$ & gag & pol & $\Delta e n v$ \\
\hline
\end{tabular}

\section{pSV-A-MLV-env}

\begin{tabular}{|c|c|c|c|}
\hline SV40 & DLTR & A-MLV env & p \\
\hline
\end{tabular}

\section{B.}

pLGPS

Tf

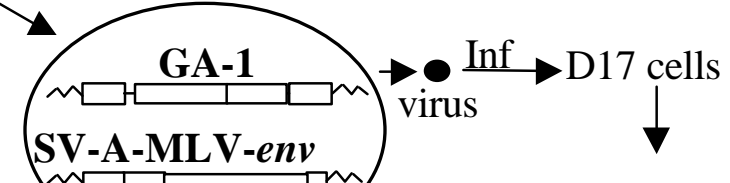

SV-A-MLV-env

ANGIE P

Determine Frequency of lacZ inactivation
Select G418 ${ }^{\mathrm{R}}$ clones

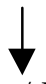

$\longleftarrow$ Stain w/ X-Gal

\section{C.}

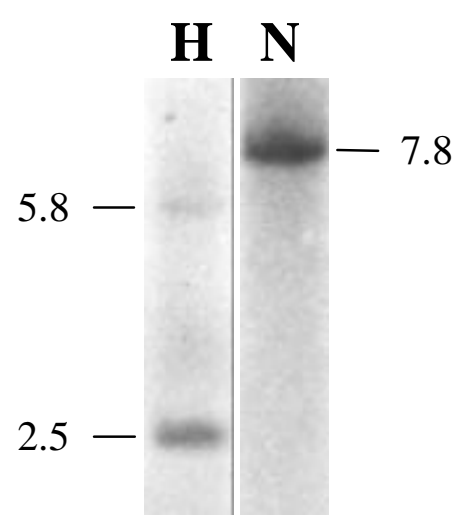


FIG. 2. Comparison of RT activities and viral titers. (A) Representative Western blot analysis of the wildtype and V223 mutants used to normalize the amount of viral proteins used in the RT assays. (B) Reverse transcriptase activity using virion-associated RT. The activities shown are relative to the RT activity determined for the wildtype MLV RT (set to $100 \%$ ). The results represent an average of 2 to 3 experiments. The error bars represent the standard error of the mean. (C) Summary of virus titers obtained relative to the wildtype viral titer (set to 100\%). The results represent an average of 2 to 10 experiments and the error bars represent the standard error of the mean. 
A.

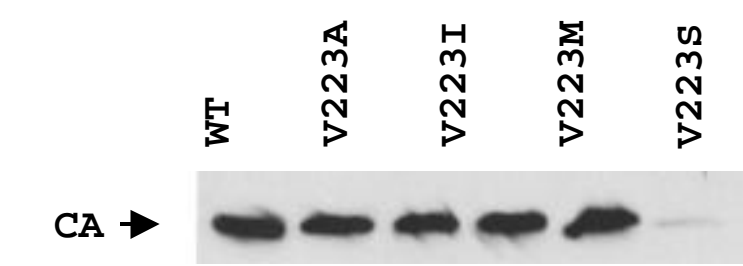

B.

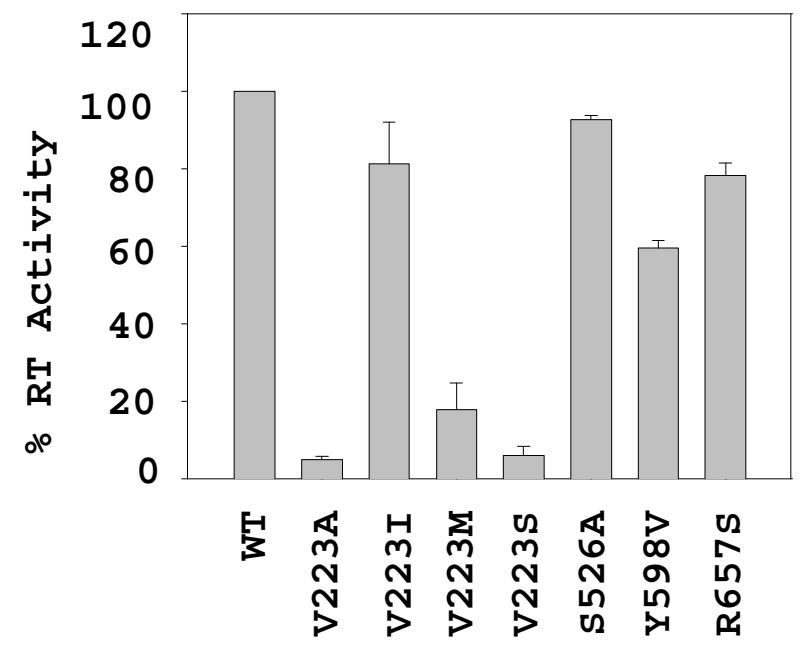

C.

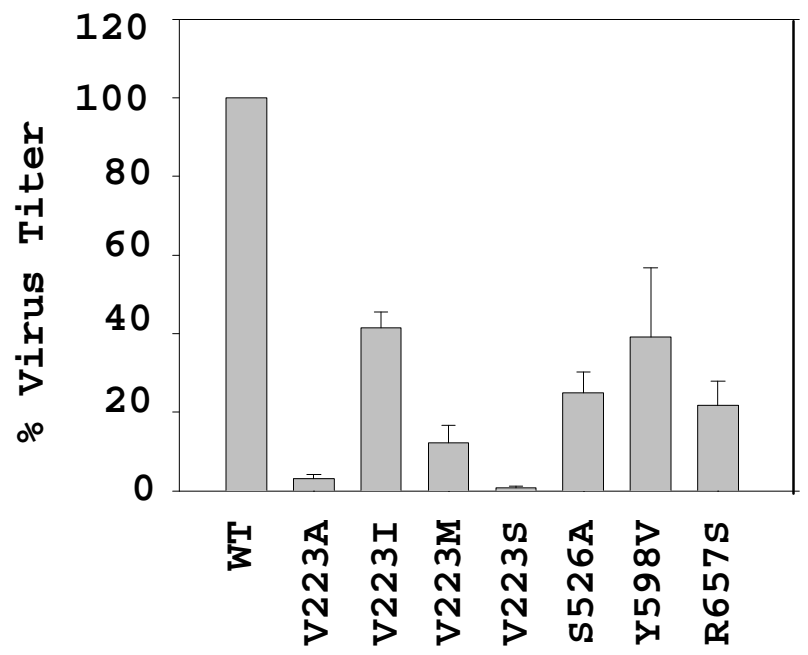


FIG. 3. PCR analysis of lacZ mutations in proviral DNAs of infected cell clones. (A) Structure of lacZ and approximate locations of the overlapping PCR amplified products. The approximate locations of 5', middle, and 3' fragments generated during PCR amplification of lac $Z$ are shown. (B) Representative restriction digestion analysis of PCR products obtained from infected cell clone $6 \mathrm{~W}$. The 5' and 3' fragments were digested with BstUI (B), HaeIII (Ha), and HhaI (Hh). The middle fragment was digested with DpnII (D), HpaII (Hp), and NlaIII (N). The odd numbered lanes represent PCR products from a cell clone containing wildtype lacZ. The even numbered lanes represent PCR products derived from cell clone $6 \mathrm{~W}$ containing an inactivated lacZ. (C) lacZ inactivating mutations in infected cell clones 1W (3330-3343 nt), 2W (3666-3680 nt), and 3W (4085$4099 \mathrm{nt})$. Inactivating mutations are shown in bold type. A +1 frameshift mutation in a run of 6 A nucleotides is shown (labeled $+\mathrm{A})$. A substitution mutation ( $\mathrm{C}$ to $\mathrm{T})$ is shown (labeled T). 
A.

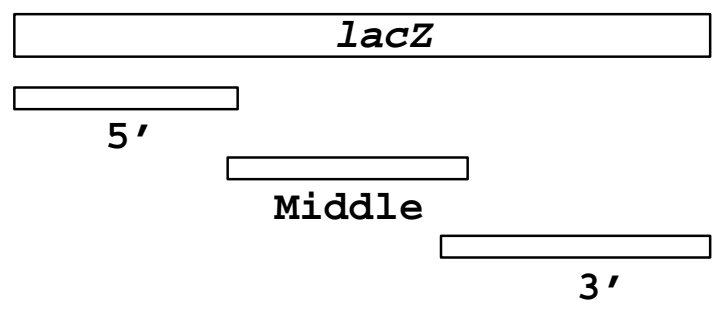

B.

$\frac{5^{\prime}}{\text { B } \text { Ha } \text { Hh }} \frac{\text { Middle }}{\text { D } \text { Hp }} \frac{3^{\prime}}{\text { B }}$

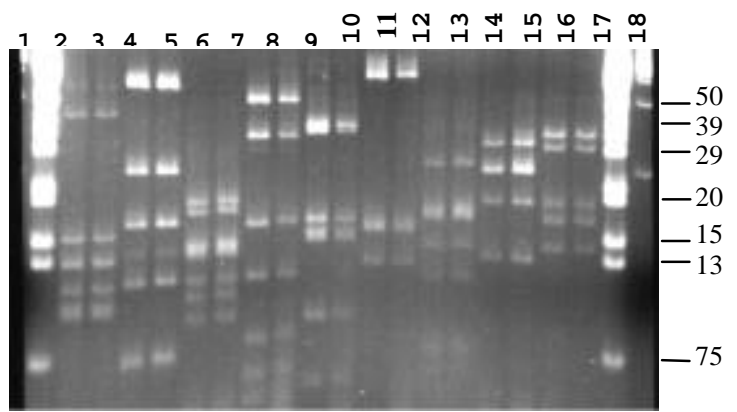

C.

\begin{tabular}{|c|c|c|c|}
\hline $1 \mathrm{~W}$ & 3330 & CCATCAAAAAA TGGC & 3344 \\
\hline & 3666 & $\stackrel{\mathbf{T}}{\text { ATCCGGGCAACCAT }}$ & 3680 \\
\hline & & GCGATA $\frac{+\mathbf{A}}{\bar{A} \bar{A} \bar{A} A} \mathrm{AAC}$ & \\
\hline
\end{tabular}




\section{REFERENCES}

1. Avidan, O., and A. Hizi. 1998. The processivity of DNA synthesis exhibited by drug-resistant variants of human immunodeficiency virus type-1 reverse transcriptase. Nucleic Acids Res. 26:1713-1717.

2. Bakhanashvili, M., O. Avidan, and A. Hizi. 1996. Mutational studies of human immunodeficiency virus type 1 reverse transcriptase: the involvement of residues 183 and 184 in the fidelity of DNA synthesis. FEBS Lett. 391:257-262.

3. Beard, W. A., K. Bebenek, T. A. Darden, L. Li, R. Prasad, T. A. Kunkel, and S. H. Wilson. 1998. Vertical-scanning mutagenesis of a critical tryptophan in the minor groove binding track of HIV-1 reverse transcriptase. Molecular nature of polymerase-nucleic acid interactions. J. Biol. Chem. 273:30435-30442.

4. Bebenek, K., J. Abbotts, J. D. Roberts, S. H. Wilson, and T. A. Kunkel. 1989. Specificity and mechanism of error-prone replication by human immunodeficiency virus-1 reverse transcriptase. J. Biol. Chem. 264:16948-16956.

5. Bebenek, K., J. Abbotts, S. H. Wilson, and T. A. Kunkel. 1993. Error-prone polymerization by HIV-1 reverse transcriptase. Contribution of template-primer misalignment, miscoding, and termination probability to mutational hot spots. J. Biol. Chem. 268:10324-10334.

6. Bebenek, K., W. A. Beard, J. R. Casas-Finet, H. R. Kim, T. A. Darden, S. H. Wilson, and T. A. Kunkel. 1995. Reduced frameshift fidelity and processivity of HIV-1 reverse transcriptase mutants containing alanine substitutions in helix $\mathrm{H}$ of the thumb subdomain. J. Biol. Chem. 270:19516-19523. 
7. Blain, S. W., and S. P. Goff. 1995. Effects on DNA synthesis and translocation caused by mutations in the RNase $\mathrm{H}$ domain of Moloney murine leukemia virus reverse transcriptase. J. Virol. 69:4440-4452.

8. Blain, S. W., and S. P. Goff. 1993. Nuclease activities of Moloney murine leukemia virus reverse transcriptase. Mutants with altered substrate specificities. J. Biol. Chem. 268:23585-23592.

9. Boucher, C. A., N. Cammack, P. Schipper, R. Schuurman, P. Rouse, M. A. Wainberg, and J. M. Cameron. 1993. High-level resistance to (-) enantiomeric 2'-deoxy-3'-thiacytidine in vitro is due to one amino acid substitution in the catalytic site of human immunodeficiency virus type 1 reverse transcriptase. Antimicrob. Agents Chemother. 37:2231-2234.

10. Boyer, P. L., and S. H. Hughes. 1995. Analysis of mutations at position 184 in reverse transcriptase of human immunodeficiency virus type 1. Antimicrob. Agents Chemother. 39:1624-1628.

11. Cepko, C. 1992. XGAL staining of cultured cells. Greene Publishing Associates and Wiley Interscience, New York, N.Y.

12. Chao, S. F., V. L. Chan, P. Juranka, A. H. Kaplan, R. Swanstrom, and C. A. Hutchison, 3rd. 1995. Mutational sensitivity patterns define critical residues in the palm subdomain of the reverse transcriptase of human immunodeficiency virus type 1. Nucleic Acids Res. 23:803-810.

13. Chesebro, B., W. Britt, L. Evans, K. Wehrly, J. Nishio, and M. Cloyd. 1983. Characterization of monoclonal antibodies reactive with murine leukemia viruses: 
use in analysis of strains of friend MCF and Friend ecotropic murine leukemia virus. Virology 127:134-148.

14. Coffin, J. M. 1995. HIV population dynamics in vivo: implications for genetic variation, pathogenesis, and therapy. Science 267:483-489.

15. Cohen, J. 1997. The daunting challenge of keeping HIV suppressed. Science 277:32-33.

16. Delviks, K. A., W. S. Hu, and V. K. Pathak. 1997. Psi- vectors: murine leukemia virus-based self-inactivating and self- activating retroviral vectors. J. Virol. 71:6218-6224.

17. Ding, J., S. H. Hughes, and E. Arnold. 1997. Protein-nucleic acid interactions and DNA conformation in a complex of human immunodeficiency virus type 1 reverse transcriptase with a double- stranded DNA template-primer. Biopolymers 44:125-138.

18. Dong, Q., W. C. Copeland, and T. S. Wang. 1993. Mutational studies of human DNA polymerase alpha. Identification of residues critical for deoxynucleotide binding and misinsertion fidelity of DNA synthesis. J. Biol. Chem. 268:2416324174.

19. Drosopoulos, W. C., and V. R. Prasad. 1998. Increased misincorporation fidelity observed for nucleoside analog resistance mutations M184V and E89G in human immunodeficiency virus type 1 reverse transcriptase does not correlate with the overall error rate measured in vitro. J. Virol. 72:4224-4230.

20. Gao, Q., Z. Gu, M. A. Parniak, J. Cameron, N. Cammack, C. Boucher, and M. A. Wainberg. 1993. The same mutation that encodes low-level human 
immunodeficiency virus type 1 resistance to 2',3'-dideoxyinosine and 2',3'-

dideoxycytidine confers high-level resistance to the (-) enantiomer of 2',3'-dideoxy3'- thiacytidine. Antimicrob. Agents Chemother. 37:1390-1392.

21. Garces, J., and R. Wittek. 1991. Reverse-transcriptase-associated RNaseH activity mediates template switching during reverse transcription in vitro. Proc. R. Soc. Lond. B. Biol. Sci. 243:235-239.

22. Georgiadis, M. M., S. M. Jessen, C. M. Ogata, A. Telesnitsky, S. P. Goff, and W. A. Hendrickson. 1995. Mechanistic implications from the structure of a catalytic fragment of Moloney murine leukemia virus reverse transcriptase. Structure 3:879-892.

23. Grandgenett, D. P., G. F. Gerard, and M. Green. 1973. A single subunit from avian myeloblastosis virus with both RNA-directed DNA polymerase and ribonuclease H activity. Proc. Natl. Acad. Sci. USA 70:230-234.

24. Guo, J., W. Wu, Z. Y. Yuan, K. Post, R. J. Crouch, and J. G. Levin. 1995. Defects in primer-template binding, processive DNA synthesis, and RNase H activity associated with chimeric reverse transcriptases having the murine leukemia virus polymerase domain joined to Escherichia coli RNase H. Biochemistry 34:5018-5029.

25. Hamburgh, M. E., W. C. Drosopoulos, and V. R. Prasad. 1998. The influence of 3TC-resistance mutations E89G and M184V in the human immunodeficiency virus reverse transcriptase on mispair extension efficiency. Nucleic Acids Res. 26:4389-4394. 
26. Harris, D., N. Kaushik, P. K. Pandey, P. N. Yadav, and V. N. Pandey. 1998. Functional analysis of amino acid residues constituting the dNTP binding pocket of HIV-1 reverse transcriptase. J. Biol. Chem. 273:33624-33634.

27. Harris, D., P. N. Yadav, and V. N. Pandey. 1998. Loss of polymerase activity due to Tyr to Phe substitution in the YMDD motif of human immunodeficiency virus type-1 reverse transcriptase is compensated by Met to Val substitution within the same motif. Biochemistry 37:9630-9640.

28. Higuchi, R. 1989. Simple and rapid preparation of samples for PCR. Stockton Press, New York.

29. Huang, H., R. Chopra, G. L. Verdine, and S. C. Harrison. 1998. Structure of a covalently trapped catalytic complex of HIV-1 reverse transcriptase: implications for drug resistance. Science 282:1669-1675.

30. Jacobo-Molina, A., J. Ding, R. G. Nanni, A. D. Clark, Jr., X. Lu, C. Tantillo, R. L. Williams, G. Kamer, A. L. Ferris, P. Clark, and et al. 1993. Crystal structure of human immunodeficiency virus type 1 reverse transcriptase complexed with double-stranded DNA at 3.0 A resolution shows bent DNA. Proc. Natl. Acad. Sci. USA 90:6320-6324.

31. Jang, S. K., H. G. Krausslich, M. J. Nicklin, G. M. Duke, A. C. Palmenberg, and E. Wimmer. 1988. A segment of the 5' nontranslated region of encephalomyocarditis virus RNA directs internal entry of ribosomes during in vitro translation. J. Virol. 62:2636-2643.

32. Jonckheere, H., M. Witvrouw, E. De Clercq, and J. Anne. 1998. Lamivudine resistance of HIV type 1 does not delay development of resistance to 
nonnucleoside HIV type 1-specific reverse transcriptase inhibitors as compared with wild-type HIV type 1. AIDS Res. Hum. Retroviruses 14:249-253.

33. Julias, J. G., D. Hash, and V. K. Pathak. 1995. E- vectors: development of novel self-inactivating and self-activating retroviral vectors for safer gene therapy. J. Virol. 69:6839-6846.

34. Julias, J. G., T. Kim, G. Arnold, and V. K. Pathak. 1997. The antiretrovirus drug 3'-azido-3'-deoxythymidine increases the retrovirus mutation rate. J Virol. 71:4254-4263.

35. Kaushik, N., K. Singh, I. Alluru, and M. J. Modak. 1999. Tyrosine 222, a member of the YXDD motif of MuLV RT, is catalytically essential and is a major component of the fidelity center. Biochemistry 38:2617-2627.

36. Kent, R. B., J. R. Emanuel, Y. Ben Neriah, R. Levenson, and D. E. Housman. 1987. Ouabain resistance conferred by expression of the cDNA for a murine Na+, K+-ATPase alpha subunit. Science 237:901-903.

37. Kim, B., T. R. Hathaway, and L. A. Loeb. 1998. Fidelity of mutant HIV-1 reverse transcriptases: interaction with the single-stranded template influences the accuracy of DNA synthesis. Biochemistry 37:5831-5839.

38. Kohlstaedt, L. A., J. Wang, J. M. Friedman, P. A. Rice, and T. A. Steitz. 1992. Crystal structure at 3.5 A resolution of HIV-1 reverse transcriptase complexed with an inhibitor. Science 256:1783-1790.

39. Kunkel, T. A., R. K. Hamatake, J. Motto-Fox, M. P. Fitzgerald, and A. Sugino. 1989. Fidelity of DNA polymerase I and the DNA polymerase I-DNA primase complex from Saccharomyces cerevisiae. Mol. Cell. Biol. 9:4447-4458. 
40. Landau, N. R., K. A. Page, and D. R. Littman. 1991. Pseudotyping with human T-cell leukemia virus type I broadens the human immunodeficiency virus host range. J. Virol. 65:162-169.

41. Luo, G. X., and J. Taylor. 1990. Template switching by reverse transcriptase during DNA synthesis. J. Virol. 64:4321-4328.

42. Mansky, L. M., and H. M. Temin. 1995. Lower in vivo mutation rate of human immunodeficiency virus type 1 than that predicted from the fidelity of purified reverse transcriptase. J. Virol. 69:5087-5094.

43. Mansky, L. M., and H. M. Temin. 1994. Lower mutation rate of bovine leukemia virus relative to that of spleen necrosis virus. J. Virol. 68:494-499.

44. Martinez, M. A., J. P. Vartanian, and S. Wain-Hobson. 1994. Hypermutagenesis of RNA using human immunodeficiency virus type 1 reverse transcriptase and biased dNTP concentrations. Proc. Natl. Acad. Sci. USA 91:11787-11791.

45. Martin-Hernandez, A. M., M. Gutierrez-Rivas, E. Domingo, and L. Menendez-Arias. 1997. Mispair extension fidelity of human immunodeficiency virus type 1 reverse transcriptases with amino acid substitutions affecting Tyr115. Nucleic Acids Res. 25:1383-1389.

46. McMichael, A. J., and R. E. Phillips. 1997. Escape of human immunodeficiency virus from immune control. Annu. Rev. Immunol. 15:271-296.

47. Miller, A. D., and C. Buttimore. 1986. Redesign of retrovirus packaging cell lines to avoid recombination leading to helper virus production. Mol. Cell. Biol. 6:2895-2902. 
48. Miller, A. D., J. V. Garcia, N. von Suhr, C. M. Lynch, C. Wilson, and M. V. Eiden. 1991. Construction and properties of retrovirus packaging cells based on gibbon ape leukemia virus. J. Virol. 65:2220-2224.

49. Nijhuis, M., R. Schuurman, D. de Jong, R. van Leeuwen, J. Lange, S. Danner, W. Keulen, T. de Groot, and C. A. Boucher. 1997. Lamivudineresistant human immunodeficiency virus type 1 variants (184V) require multiple amino acid changes to become co-resistant to zidovudine in vivo. J. Infect. Dis. 176:398-405.

50. Oude Essink, B. B., N. K. Back, and B. Berkhout. 1997. Increased polymerase fidelity of the 3TC-resistant variants of HIV-1 reverse transcriptase. Nucleic Acids Res. 25:3212-3217.

51. Pandey, V. N., N. Kaushik, N. Rege, S. G. Sarafianos, P. N. Yadav, and M. J. Modak. 1996. Role of methionine 184 of human immunodeficiency virus type-1 reverse transcriptase in the polymerase function and fidelity of DNA synthesis. Biochemistry. 35:2168-2179.

52. Parthasarathi, S., A. Varela-Echavarria, Y. Ron, B. D. Preston, and J. P. Dougherty. 1995. Genetic rearrangements occurring during a single cycle of murine leukemia virus vector replication: characterization and implications. J. Virol. 69:7991-8000.

53. Pathak, V. K., and H. M. Temin. 1990. Broad spectrum of in vivo forward mutations, hypermutations, and mutational hotspots in a retroviral shuttle vector after a single replication cycle: deletions and deletions with insertions. Proc. Natl. Acad. Sci. USA 87:6024-6028. 
54. Pathak, V. K., and H. M. Temin. 1990. Broad spectrum of in vivo forward mutations, hypermutations, and mutational hotspots in a retroviral shuttle vector after a single replication cycle: substitutions, frameshifts, and hypermutations. Proc. Natl. Acad. Sci. USA 87:6019-6023.

55. Preston, B. D., B. J. Poiesz, and L. A. Loeb. 1988. Fidelity of HIV-1 reverse transcriptase. Science 242:1168-1171.

56. Rezende, L. F., K. Curr, T. Ueno, H. Mitsuya, and V. R. Prasad. 1998. The impact of multidideoxynucleoside resistance-conferring mutations in human immunodeficiency virus type 1 reverse transcriptase on polymerase fidelity and error specificity. J. Virol. 72:2890-2895.

57. Rubinek, T., M. Bakhanashvili, R. Taube, O. Avidan, and A. Hizi. 1997. The fidelity of 3' misinsertion and mispair extension during DNA synthesis exhibited by two drug-resistant mutants of the reverse transcriptase of human immunodeficiency virus type 1 with Leu74-->Val and Glu89-->Gly. Eur. J. Biochem. 247:238-247.

58. Sambrook, J., E. F. Fritsch, and T. Maniatis. 1989. Molecular cloning: a laboratory manual, 2nd ed. Cold Spring Harbor Laboratory, Cold Spring Harbor, N.Y.

59. Tantillo, C., J. Ding, A. Jacobo-Molina, R. G. Nanni, P. L. Boyer, S. H. Hughes, R. Pauwels, K. Andries, P. A. Janssen, and E. Arnold. 1994. Locations of anti-AIDS drug binding sites and resistance mutations in the threedimensional structure of HIV-1 reverse transcriptase. Implications for mechanisms of drug inhibition and resistance. J. Mol. Biol. 243:369-387. 
60. Taube, R., O. Avidan, and A. Hizi. 1997. The fidelity of misinsertion and mispair extension throughout DNA synthesis exhibited by mutants of the reverse transcriptase of human immunodeficiency virus type 2 resistant to nucleoside analogs. Eur. J. Biochem. 250:106-114.

61. Telesnitsky, A., and S. P. Goff. 1993. RNase H domain mutations affect the interaction between Moloney murine leukemia virus reverse transcriptase and its primer-template. Proc. Natl. Acad. Sci. USA 90:1276-1280.

62. Temin, H. M. 1993. Retrovirus variation and reverse transcription: abnormal strand transfers result in retrovirus genetic variation. Proc. Natl. Acad. Sci. USA 90:6900-6903.

63. Tisdale, M., S. D. Kemp, N. R. Parry, and B. A. Larder. 1993. Rapid in vitro selection of human immunodeficiency virus type 1 resistant to 3 '-thiacytidine inhibitors due to a mutation in the YMDD region of reverse transcriptase. Proc. Natl. Acad. Sci. USA 90:5653-5656.

64. van der Groen, G., P. N. Nyambi, E. Beirnaert, D. Davis, K. Fransen, L. Heyndrickx, P. Ondoa, G. Van der Auwera, and W. Janssens. 1998. Genetic variation of HIV type 1: relevance of interclade variation to vaccine development. AIDS. Res. Hum. Retroviruses 14 Suppl 3:S211-21.

65. Vara, J. A., A. Portela, J. Ortin, and A. Jimenez. 1986. Expression in mammalian cells of a gene from Streptomyces alboniger conferring puromycin resistance. Nucleic Acids Res. 14:4617-4624.

66. Wainberg, M. A., W. C. Drosopoulos, H. Salomon, M. Hsu, G. Borkow, M. Parniak, Z. Gu, Q. Song, J. Manne, S. Islam, G. Castriota, and V. R. Prasad. 
1996. Enhanced fidelity of 3TC-selected mutant HIV-1 reverse transcriptase.

Science 271:1282-1285.

67. Wakefield, J. K., S. A. Jablonski, and C. D. Morrow. 1992. In vitro enzymatic activity of human immunodeficiency virus type 1 reverse transcriptase mutants in the highly conserved YMDD amino acid motif correlates with the infectious potential of the proviral genome. J. Virol. 66:6806-6812. 


\title{
CHAPTER 3
}

\section{Residues of the dNTP-Binding Site of Murine Leukemia Virus Reverse Transcriptase that are Important for In Vivo Fidelity, Reverse Transcription, and Retroviral Replication}

\begin{abstract}
Elias K. Halvas, ${ }^{1}$ Evguenia S. Svarovskaia, ${ }^{1,2}$ and Vinay K. Pathak ${ }^{2^{*}}$
Mary Babb Randolph Cancer Center and Department of Biochemistry, West Virginia University, Morgantown, West Virginia 26506, ${ }^{1}$ and HIV Drug Resistance Program, National Cancer Institute, FCRDC, Frederick, Maryland $21702^{2}$
\end{abstract}

Running Title: MLV RT fidelity and the putative dNTP binding site

This manuscript was submitted for publication to the Journal of Virology, May 2000 


\begin{abstract}
Retroviral populations exhibit a high evolutionary potential giving rise to extensive genetic variation. A mechanism that generates variation in retroviral populations may be error-prone DNA synthesis catalyzed by reverse transcriptase (RT). Structural features within RTs are likely to contribute to the high rate of errors that occur during reverse transcription. We sought to determine whether amino acids within murine leukemia virus (MLV) RT that contact the deoxyribonucleotide triphosphate (dNTP) substrate are important for fidelity of reverse transcription in vivo. We utilized the previously described ANGIE $P$ encapsidating cell line, which expresses the amphotropic MLV envelope and a retroviral vector (pGA-1). pGA-1 expresses the bacterial $\beta$-galactosidase gene (lacZ), which serves as a reporter of mutations. Extensive mutagenesis was performed on residues likely to interact with the dNTP substrate and the effects of these mutations on the fidelity of reverse transcription were determined. As expected, most substitution mutations at positions believed to directly interact with the dNTP substrate significantly reduced viral titers $(>10,000$-fold $)$, indicating that these residues played a critical role in catalysis and viral replication. However, the D153A and A154S substitutions, which are predicted to affect the interactions with the triphosphate, resulted in statistically significant increases in the mutation rate. In addition, the conservative substitution F155W, which may affect interactions with the base and the ribose, increased the mutation rate 2.8 -fold. Substitutions at residues in the vicinity of the dNTP-binding site also resulted in statistically significant decreases in fidelity (1.3- to 2.4-fold). These results suggest that
\end{abstract}


mutations of residues that contact the substrate dNTP are more likely to prevent viral replication than to alter the fidelity of $\mathrm{RT}$.

\section{INTRODUCTION}

Retroviral populations display extensive genetic variability that can be attributed to properties of the virally encoded reverse transcriptase (RT). The innate ability of RT to be less processive than most polymerases and/or the lack of a $3^{\prime}-5^{\prime}$ exonuclease activity (proofreading mechanism), may allow for the reduction of accurate DNA synthesis $(37,42)$. It has been observed in vivo that at least $1 / 3$ of the generated mutations occur during the plus-stand DNA synthesis of reverse transcription (28). Therefore, errors generated by RT may contribute to the variation in viral populations as seen with human immunodeficiency virus type 1 (HIV-1) (5). Several amino acid motifs present in different retroviral RTs have been identified that may exert an impact on the fidelity of DNA synthesis during reverse transcription. These include residues of the Tyr-X-Asp-Asp (YXDD) motif, the deoxyribonucleotide triphosphate (dNTP) binding site, the $\alpha \mathrm{H}$ helix of the thumb domain, the conserved Leu-Pro-Gln-Gly (LPQG) motif, the fingers domain, as well as specific amino acids such as E89 and F160 in HIV-1 RT $(1,3,4,9,11,14-16,26,33,39,47)$. The mechanism by which fidelity is maintained may encompass subtle features in RT that involve the local geometry of the active site, the proper positioning of the template-primer complex, or the global effects attributed to different conformational states of the protein $(7,16)$.

It was previously shown that residues 110 to 116 in HIV-1 RT are located in the active site of RT and have a moderate to high solvent accessibility, suggesting that they may play a role in dNTP binding $(20,41)$. Furthermore, extensive mutational analysis of 
residues Y115 and Q151 in HIV-1 RT revealed that substitutions could have drastic effects on substrate dNTP binding $(33,39)$ as well as play a role in resistance to nucleoside analogs $(19,43)$. Resistance to nucleoside analogs has also been observed with position 116 as well (45). Additional evidence for the importance of this stretch of residues in dNTP binding and catalysis was provided by the ability of the D113 and A114 mutants of HIV-1 RT that exhibit a decrease in the sensitivity to phosphonoformic acid (PFA), a pyrophosphate analog (31). It was hypothesized that these residues, in addition to the highly conserved R72, play an important role during pyrophosphate exchange (31, 40). Finally, mutations at K65 have implicated this residue in template-primer complex binding that may ultimately have effects on fidelity $(13,29,41)$.

Recently, the structure of a ternary complex of HIV-1 RT catalytically trapped with both template-primer and deoxyribonucleotide thymidine triphosphate (dTTP) substrates was elucidated, which identified the residues that directly contacted the incoming dNTP (18). These included residues K65, R72, D113, A114, Y115, and Q151 of HIV-1 RT. The K65 and R72 side chains as well as the D113 and A114 main chain amide nitrogens (NH) bind to the $\alpha, \beta$ and $\gamma$ phosphates of the incoming dTTP through hydrogen bonding interactions. In addition, it has been illustrated that the side chains of R72, Y115, and Q151 interact with the base of the dTTP. Finally, the main chain NH of residue Y115 was also shown to be in close proximity to the ribose moiety of the dTTP (18).

Since substrate dTTP and the aforementioned amino acids are in close proximity to each other, it is reasonable to hypothesize that substitutions at these positions may alter the binding of the incoming dNTP, positioning of the template-primer complex, or modify the local geometry of the substrate binding pocket (16). This alteration in turn 
can lead to changes in fidelity. This hypothesis is supported by evidence from mutations introduced at positions K65 and Q151 of recombinant HIV-1 RT, which exhibited decreased mispair extension in vitro as well as resistance to a number of dideoxynucleoside analogs such as $2^{\prime}, 3^{\prime}$-dideoxy-3'-thiacytidine (3TC), $3^{\prime}$-azido- $3^{\prime}$ deoxythymidine (AZT), 2',3'-dideoxycytidine (ddC), and 2',3'-dideoxyinosine (ddI) (12, 16, 44). Finally, a number of mutations introduced at residue Y115 of HIV-1 RT were shown to affect the frequency of misinsertions and mispair extensions $(33,34)$.

Primary sequence and crystal structure data can be utilized to identify amino acids in murine leukemia virus (MLV) RT that are equivalent to those in HIV-1 RT. Sequence comparisons of several RTs illustrate the presence of highly conserved amino acid motifs (36). The partial structure of the fingers and palm subdomains of MLV RT has been solved (11). Comparison of the fingers and thumb subdomains of HIV-1 and MLV RTs reveal similar tertiary structures despite having only $\sim 25 \%$ amino acid sequence identity (11, 17). Furthermore, MLV RT possesses several of the structural motifs present in HIV-1 RT, including the conserved YXDD, the Thr-Val-Leu-Asp (TVLD), and LPQG motifs $(11,36)$. Sequence alignment of common amino acid motifs within the two RTs and a comparison of the crystal structures reveal MLV RT residues that are homologous to those in HIV-1 RT $(11,17)$. Such comparisons strongly suggest that residues K103, R110, D153, A154, F155, and Q190 of MLV RT are equivalent to the dTTP binding residues K65, R72, D113, A114, Y115, and Q151 in HIV-1 RT, respectively. Furthermore, K103 of MLV RT has been observed to interact with the incoming dNTP, and substitutions at R110 affect processivity $(2,7)$. It has been postulated that F155 of MLV RT may be involved in the selection of dNTPs over ribonucleotides (rNTPs) due to 
steric hindrance between the $2^{\prime} \mathrm{OH}$ of rNTP and the F155 side chain (11). More recent studies have provided direct evidence that the F155V mutant of MLV RT is able to incorporate rNTPs during polymerization (10).

In this study, we sought to determine whether alterations in the structure of MLV RT could affect the fidelity of reverse transcription in vivo. Specifically, we examined whether mutations at residues in the vicinity of the dNTP binding pocket as well as residues that directly contact the substrate dNTP could alter the fidelity of reverse transcription in vivo. We assessed the effects of these mutations on replication, polymerase activity, and fidelity by comparing the mutant MLV RTs to wild-type RT.

\section{MATERIAL AND METHODS}

Plasmids and retroviral vectors. The construction of the MLV-based retroviral vector pGA-1 was previously described (23). The vector pGA-1 possesses cis-acting elements needed for viral replication. In addition, the vector expresses the bacterial $\beta$ galactosidase gene (lacZ) and the neomycin phosphotransferase gene (neo) from a single RNA transcript initiating from the viral promoter. An internal ribosomal entry site (IRES) of encephalomyocarditis virus is used to insure for the translation of neo (21). Plasmid pLGPS expresses the MLV gag and pol genes from a truncated long terminal repeat $(\triangle \mathrm{LTR})$ promoter $(35)$. Plasmid $\mathrm{pRMBNB}$, which was derived from pLGPS, contains three unique restriction sites (Bst1107I, NsiI, and BstBI) near the dNTP-binding site of MLV RT. These three restriction sites were generated by the introduction of silent substitutions to facilitate further mutagenesis. The plasmid pSV-A-MLV-env, which expresses the amphotropic MLV envelope gene from the LTR promoter and SV40 enhancer, was obtained from the NIH AIDS Research and Reference Reagents Program 
(30). Plasmid pBSpac encodes the puromycin N-acetyltransferase gene and therefore confers resistance to puromycin (46). Plasmid pSV $\alpha 3.6$ encodes the $\alpha$ subunit of the murine $\mathrm{Na}^{+}, \mathrm{K}^{+}$-ATPase gene and confers resistance to ouabain (25).

Generation of dNTP-binding site MLV RT mutants. A detailed description of the mutagenic oligonucleotides and strategies used to generate as well as identify mutants is available upon request. Briefly, pRMBNB was generated by two rounds of site-directed mutagenesis using the Chameleon kit (Stratagene) with mutagenic primers and pLGPS as the template. pRMBNB was screened by the presence of three unique restriction sites, Bst1107I, NsiI, and BstB1. To ensure the absence of any undesired mutations, the $B c l$ I to SalI DNA fragment of pRMBNB was subcloned into pLGPS. In addition, the Q190E, Q190H, and Q190N mutants were generated in a similar manner using the Chameleon kit (Stratagene) with either pLGPS or pRMBNB as the templates and the appropriate mutagenic primers. The Q190E mutant was generated utilizing pLGPS as the template and screened by the creation of a new Bsu36I site. The BclI to SalI DNA fragment of Q190E was subcloned into pLGPS to ensure the absence of any undesired mutations. The Q190E mutant, designated as pESQ190E was also generated in pRMBNB and screened by the creation of a new Bsu36I site. Both the Q190H and Q190N mutants were generated using the Chameleon kit (Stratagene) with pESQ190E as the template and screened by the loss of the new Bsu36I site. The Nsi to SalI DNA fragments of Q190H and Q190N were subcloned into pRMBNB to ensure the absence of any undesired mutations.

PCR-based mutagenesis with random mutagenic primer sets and pRMBNB as the template was utilized to generate mutants at residues K103 and R110. Amplified DNA fragments of 493 bp were digested with $B c l$ I and $N s i$ I and subcloned back into pRMBNB. 
In addition, the Q190M mutant was generated in a similar manner using PCR-based mutagenesis utilizing mutagenic primers and screened by sequencing. An amplified DNA fragment of 831 bp was digested with NsiI and SalI and subcloned back into pRMBNB.

Mutations introduced at positions 147 to 160 of MLV RT were generated by double-stranded DNA oligonucleotide (dsDNA oligo)-based mutagenesis. These various dsDNA oligos, which are complementary to the Bst1107I to BstBI fragment in pRMBNB and contain the appropriate mutations, were subcloned back into pRMBNB. One or two silent mutations were also introduced into these oligonucleotides to generate new restriction sites to facilitate identification of mutant plasmids. Mutations introduced at positions 147 to 153 of MLV RT were generated by replacing the Bst1107I to NsiI fragment of pRMBNB with 25-bp dsDNA oligos. Mutations introduced at positions 154 to 155 were generated by replacing the Bst $1107 \mathrm{I}$ to $B s t \mathrm{BI}$ fragment of pRMBNB with 73bp dsDNA oligos. Mutations introduced at positions 155 to 160 were generated by replacing the $N$ si I to $B s t$ BI fragment of pRMBNB with 48-bp dsDNA oligos. Mutants were identified by digestion with either $\mathrm{XbaI}$ (mutants at positions 147 to 155) or SpeI (mutants at positions 156 to160).

Finally, all mutated plasmids were mapped extensively utilizing various restriction enzymes. In addition, mutated plasmids with inserted fragments containing the appropriate mutations, were analyzed by DNA sequencing (ALF Automated Sequencer, Pharmacia) to verify the presence of the desired mutation and the absence of any undesired mutations.

Cells, transfections, and infections. D17 dog osteosarcoma cells were transfected with either the MLV-based vector or expression constructs or infected with 
MLV and selected for resistance to ouabain or G418 (a neomycin analog) as previously described $(22,23)$. The D17-based ANGIE P cells were maintained, transfected, and selected for drug resistance in a similar manner (15).

Assay for determining in vivo fidelity. The ANGIE P cells were plated at a density of $2 \times 10^{5}$ cells per 60 -mm-diameter dish, and $24 \mathrm{~h}$ later were cotransfected with wild type or mutated pLGPS and $\mathrm{pSV} \alpha 3.6$. The transfected cells were selected for

resistance to ouabain $\left(10^{-7} \mathrm{M}\right)$, then resistant colonies were pooled, expanded, and plated at a density of $5 \times 10^{6}$ cells per 100 -mm-diameter dish. After $48 \mathrm{~h}$, the culture medium containing GA-1 virus was harvested and used to infect D17 target cells plated at a density of $2 \times 10^{5}$ cells per 60 -mm-diameter dish. Infected D17 cells were selected for resistance to $\mathrm{G} 418(400 \mu \mathrm{g} / \mathrm{ml})$ and stained with 5-bromo-4-chloro-indolyl- $\beta$-Dgalactopyranoside (X-Gal) as previously described (15).

Virus preparation, RT assays, and Western blotting. Virus isolation and concentration as well as RT assays, and Western blots were performed as previously described (15). Briefly, helper cells containing different pLGPS constructs were plated at $5 \times 10^{6}$ cells per $100-\mathrm{mm}$ dish, viruses were collected 2 days later and centrifuged at $25,000 \mathrm{rpm}$ for $90 \mathrm{~min}$ in an $\mathrm{SW} 41$ rotor $\left(\right.$ Beckman) at $4^{\circ} \mathrm{C}$. Viral pellets were resuspended in phosphate-buffered saline (PBS) and virus was stored at $-80^{\circ} \mathrm{C}$.

Exogenous RT activities were determined as previously described using $50 \mu \mathrm{g} / \mu \mathrm{l}$ of (20-mer) oligo (dT) (Integrated DNA Technologies), $100 \mu \mathrm{g} / \mathrm{ml}$ of poly (rA) (Pharmacia), and $10 \mu \mathrm{Ci}$ of $\left[{ }^{3} \mathrm{H}\right] \mathrm{dTTP}$ (specific activity of $72 \mathrm{Ci} / \mathrm{mMol}, \mathrm{ICN}$ ). The amount of $\left[{ }^{3} \mathrm{H}\right]$ dTTP incorporated was determined using a scintillation counter (13). 
Western blots were used to quantify the amount of protein for the RT assays and performed using standard procedures (38). Briefly, virus was resolved using a $15 \%$ SDS-PAGE and proteins were transferred to Immobilin Membranes (Gelman Sciences, Inc.). Membranes were incubated with primary (monoclonal anti-MLV capsid IgG1; 1:10 dilution [ATCC]) (6) and secondary (anti-rat IgG antibody conjugated to horseradish peroxidase; 1:10,000 dilution [Southern Biotechnology Associates, Inc.]) antibodies and detection of MLV capsid was performed using an ECL (enhanced chemiluminenesence) kit ${ }^{\mathrm{TM}}$ (Amersham Pharmacia Biotech). Treated membranes were then exposed to X-OMAT film (Kodak) and the intensity of the p30 band was quantitated using the Image Quant program (Molecular Dynamics) (15).

\section{RESULTS}

Determination of in vivo fidelity. Construction and characterization of the ANGIE P cell line has been previously described (15). Briefly, the ANGIE P cell line expresses the MLV-based retroviral vector pGA-1 and the MLV envelope expression construct pSV-A-MLV-env (Fig. 1). The MLV gag/pol expression construct pLGPS was subjected to site-directed mutagenesis at residues constituting the dNTP-binding site of MLV RT and subsequently, wild type or mutated pLGPS was separately introduced into the ANGIE P cells by cotransfection with pSV $\alpha 3.6$. Ouabain-resistant colonies were pooled and expanded; virus was harvested from these pools and serially diluted, which was used to infect D17 target cells. Infect D17 cells resistant to G418 were selected, stained with $\mathrm{X}-\mathrm{GAL}$, and the frequency of lacZ inactivation was determined by dividing the number of white colonies by the total number of colonies (blue plus white colonies). The frequency of $l a c Z$ inactivation provided a measure of the inactivating mutations 
(white phenotype) introduced into the lacZ gene during one cycle of retroviral replication and the virus titers were used to determine the effect of mutations on the efficiency of virus replication.

Identification of the dNTP-binding site in MLV RT. Comparison of both HIV1 and MLV RT revealed the dNTP-binding site in MLV RT. Amino acid sequence alignment of HIV-1 and MLV RTs revealed the highly conserved TVLD, DAXF, and the LPQG motifs (Fig. 2A). This sequence comparison suggested that residues K103, R110, D153, A154, F155, and Q190 of MLV RT are equivalent to residues K65, R72, D113, A114, Y115, Q151 of HIV-1 RT, respectively. Analysis of the crystal structures in the context of the position and orientation of these residues also revealed similarities between the two RTs (Fig. 2B and C). In addition, bond distances calculated between residues constituting the dNTP-binding sites of both RTs (absence of ligands) (11, 17) showed that most distances are within $3 \AA$, the length of a hydrogen bond (data not shown). The only distances greater than $3 \AA$ involve the R110 position. These lengths were determined by the RasMol program and are characterized as an average bond distance calculated from a number of measurements between the various atoms of the side chain pairs inspected. The equivalence of these MLV RT residues with those in HIV-1 RT is further supported by previous studies $(2,7,11,36)$.

\section{Mutational analysis of the MLV RT dNTP-binding site and effects on}

fidelity. MLV RT was mutated at six different residues hypothesized to contact the incoming dNTP. Several conserved and non-conserved substitutions were introduced at residues K103, R110, D153, A154, F155, and Q190 of MLV RT. The effects of these various mutations on the frequency of lac $Z$ inactivation were determined and compared 
with the frequency of inactivation obtained with wild-type MLV RT in parallel experiments (Table 1). The wild-type pLGPS construct inactivated lacZ at an average frequency of $5.4 \%$ and SE $\pm 0.23 \%$ (13 independent experiments) during one cycle of retroviral replication. These results were comparable to results obtained previously (15).

As illustrated in Table 1, the majority of mutations introduced at these positions dramatically reduced viral replication, evident as a greater than 10,000-fold reduction in viral titers in comparison to the wild-type RT $\left(9.8 \times 10^{4} \mathrm{CFU} / \mathrm{ml}\right)$. Relative changes in the inactivation of lac $Z$ could therefore not be determined. Mutants with severe reductions in titer (> 10,000-fold) were observed for all dNTP-binding site positions analyzed. Our results suggest that these amino acid positions are critical for the proper function of the polymerase, consistent with previous observations showing that they are highly conserved among retroviral species (36). However, nine mutants of residues constituting the dNTP-binding site in MLV RT did permit viral replication to the extent that relative changes in fidelity could be measured. Three of the nine mutants (A154S, D153A, and F155W) exhibited increases in the frequency at which lac $Z$ was inactivated by 1.3 - to 2.8 -fold relative to the wild-type RT $(5.4 \% \pm 0.23 \%)$. These changes were shown to be statistically different from the frequency of lacZ inactivation by the wildtype RT ( $P$ values ranging from 0.03 to 0.00003 by a Two Sample T-Test). In addition, these mutations also reduced viral titers 66- to 2500-fold in comparison to wild-type RT. Conversely, six of the nine mutants (K103R, D153C, D153Q, D153S, F155Y, and Q190M) did not display a significant change in the frequency of lacZ inactivation (5.1\% $\pm 0.15 \%$ to $5.7 \% \pm 0.23 \%)$ and were shown to be similar to wild-type RT $(P>0.5)$. The K103R, D153C, D153Q, and D153S mutants also exhibited decreases in viral titers 
ranging from 25- to 1429-fold in comparison to the wild-type RT (Table 1). In contrast, the F155Y and Q190M mutants exhibited virus titers similar to wild-type MLV RT. Previous observations have shown that a 2 -fold alteration in viral titers is not believed to be significant, considering the inherent variation occurring during infections (15).

Effects of mutations at the F156 position of MLV RT on fidelity. We hypothesized that the F156 residue of MLV RT played an indirect role in substrate binding because of its proximity to other residues that constitute the dNTP-binding site. Specifically, analysis of the MLV RT crystal structure suggested that the bulky hydrophobic nature of F156 might play a role in the proper positioning of Q190, which is important for dNTP binding. Therefore, we investigated the effects of seven mutations (F156I, F156L, F156M, F156Q, F156V, F156W, and F156Y) of this residue in MLV RT on the frequency of $l a c Z$ inactivation and viral replication (Table 2). As expected, substitutions at the F156 position were tolerated to a greater extent than residues believed to be in direct contact with the incoming dNTP. Five of the seven F156 mutants replicated at levels in which their effects on fidelity could be determined. The F156L and F156M mutants exhibited increases in the frequency with which lacZ was inactivated by 1.6- and 1.7-fold relative to the wild-type RT $(5.4 \% \pm 0.25$; obtained from 8 independent experiments). These changes were shown to be statistically different from the frequency of lacZ inactivation by the wild-type RT $(P<0.001)$. Both the F156Y and F156W mutants of MLV RT displayed a slight increase in fidelity as evidenced by the frequencies of $l a c Z$ inactivation of $4.3 \% \pm 0.27 \%$ and $4.3 \% \pm 0.15 \%$, respectively when compared to wild-type MLV RT $(P<0.05)$. All of these mutants displayed reductions in viral titers relative to the wild-type RT $\left(8.1 \times 10^{4} \mathrm{CFU} / \mathrm{ml}\right)$ that ranged from 22 - to 
500-fold. Finally, the F156I, F156Q, and F156V mutants displayed reductions in virus titers greater than 10,000 fold and relative changes in fidelity for these mutants could not be determined (Table 2).

\section{Effects of mutations at residues flanking the MLV RT dNTP-binding site on}

fidelity. Regions of MLV RT (positions 147 to 152 and 157 to 161) flanking the residues of the dNTP-binding site were also subjected to mutagenesis, and the effects of these mutations on the fidelity of reverse transcription and viral replication were determined. The data obtained are summarized in Table 3. The frequencies of lac $Z$ inactivation by wild-type MLV RT obtained from six independent experiments were again highly reproducible $(5.3 \% \pm 0.26 \%)$. The H161A, T147A, C157A, K152A, and L151F mutants exhibited frequencies of lacZ inactivation that were 1.2-, 1.3-, 1.3-, 1.4-, and 2.4- fold higher than that of the wild-type RT, respectively. All five of these mutants displayed alterations in fidelity that were statistically different from that of wild-type RT ( $P$ values ranging from 0.04 to 0.000009 ). On the other hand, the R159A mutant was found to be statistically similar to wild-type RT $(P>0.05)$. The L151F and the T147A mutants displayed an 8- and 50-fold reduction in viral titers, respectively. In contrast, the K152A, C157A, and H161A mutants did not exhibit substantial changes in viral titers. Finally, viral replication of the V148D, L149W, and D150E mutants could not be detected, as evidenced by a greater than 10,000-fold reductions in viral titers. Therefore, the frequency of lacZ inactivation for these mutants could not be determined (Table 3).

RT activities of the dNTP-binding site mutant RTs. Viruses generated from either wild type or mutated pLGPS were harvested, concentrated by ultracentrifugation, and analyzed by Western blotting (data not shown). Western blots using an anti-MLV 
capsid antibody were quantified to estimate the amount of capsid protein present in the viral preparations and to ensure that equivalent amounts of viral proteins were used for determination of RT activities (summarized in Table 4).

In general, mutants (31 out of 50) possessing RT activities of less than $3 \%$ of the wild-type RT activity also had drastic reductions in viral titers (> 10,000-fold or $0.01 \%$, relative to wild type). Only the F156L mutant displayed an RT activity of $2.4 \%$ relative to wild type yet produced a low but detectable viral titer $(0.6 \%$ in comparison to wild type). In addition, the F155I and F155V mutants displayed slightly higher RT activities (4.9 and 5.5\%, respectively), but did not produce a detectable viral titer. The RT activities of the remaining 18 mutants ranged from 5.5 to $161.4 \%$ relative to the wild-type MLV RT and exhibited detectable viral titers. With the exception of the L151F, K152A, F155Y, C157A and H161A mutants, the RT activities of all mutants were statistically different from the wild-type RT activity $(P<0.002)$. Finally, the RT activity of the K103S mutant could not be compared with the wild-type RT activity, because the mutation apparently generated a defect in Gag processing and the processed capsid protein could not be detected upon Western blotting analysis (data not shown). Therefore, the RT activity of the K103S mutant could not be normalized to the wild-type RT. Finally, reductions in titers were less than decreases in RT activity, except for the Q190M mutant, suggesting that other steps in reverse transcription were affected by these mutations introduced at positions constituting the dNTP-binding site of MLV RT.

\section{DISCUSSION}

In this study, we compared the primary amino acid sequences as well as the crystal structures of MLV and HIV-1 RTs to identify residues of MLV RT that are likely 
to contact the dNTP substrate. Our extensive mutational analysis of these residues in MLV RT strongly suggests that these residues are critical for proper polymerase function and viral replication. The location and orientation of MLV RT residues D153, A154, F155, and Q190 are essentially identical to those of HIV-1 RT residues D113, A114, Y115, and Q151, respectively (Fig. 2A and 2C)(11, 17). In addition, the distances between the side chains of these residues as well as K103 are very similar to those of the equivalent residues of HIV-1 RT. The majority of the distances do not vary by more than 3.0 A. However, some of the bond distances between the R110 residue of MLV RT and the other residues constituting the dNTP-binding site are much greater than the analogous bond distances in HIV-1 RT. The R110 residue, with respect to the other five residues, appears in some cases to be as much as $4.0 \AA$ farther away and as much as $6.0 \AA$ closer (depending on which dNTP binding site residues were used to measure distances to R110) in comparison to the homologous distances in HIV-1 RT. It is possible that the MLV RT fingers domain that contains the K103 and R110 undergoes a larger movement upon binding the dNTP substrate. In addition, their location may be slightly altered in the MLV RT crystal structure, since it lacks the thumb, connection, and RNase $\mathrm{H}$ domains of MLV RT.

Several mutants of residues constituting the dNTP-binding site of MLV RT show statistically significant alterations in fidelity relative to the wild-type RT. These include D153A, A154S, and F155W, characterized by 1.6-, 1.3-, and 2.8-fold increases in lacZ inactivation, respectively (Table 1). All three homologous residues in HIV-1 RT (D113, A114, and Y115) have been observed to directly contact the incoming dTTP substrate (18) and mutations in at least the A114 and Y115 residues have been implicated in 
alterations of the affinity for the dNTP substrate (32-34). Interestingly, coordination of the triphosphate moiety of the dTTP substrate by the D113 and A114 residues of HIV-1 $\mathrm{RT}$ is believed to be mediated by the main chain amino $(\mathrm{NH})$ groups of these residues and therefore, the side chains may not have a direct effect on dNTP binding (18). This lack of possible side chain involvement might account for the increased tolerability to substitutions, at least at position D153 in MLV RT (equivalent to D113 in HIV-1 RT). However, there appears to be a limit on the side chain size that can be accommodated in this particular location due to the flexibility of the MLV RT dNTP binding pocket. For example, substitutions with amino acids containing large side chains (D153F and D153R) displayed drastic reductions in titers (> 10,000-fold) and RT activities (30- to 50-fold). In comparison, amino acids with smaller side chains (D153A, D153C, D153Q, and D153S) were more readily accepted with slightly less severe effects on viral replication and RT activity relative to the wild-type RT (Table 1). On the other hand, the change in fidelity displayed by D153A may be attributed to the small size of the side chain, which could increase the overall flexibility of the dNTP binding pocket and alter the discriminatory ability of this particular RT. One would expect a similar pattern with the A154 mutants of MLV RT. However, the majority of substitutions at this position had substantial effects on viral replication and RT activity relative to the wild-type RT (Table 1). Our data suggests that an amino acid with a side chain containing more than two carbons might alter the active site in the polymerase by repositioning critical residues important for catalysis, since only the A154S mutant seems to have detectable levels of viral replication. 
Residue Y115 in HIV-1 RT (equivalent to F155 of MLV RT) has been observed to directly interact with the base and ribose moiety of the incoming dTTP substrate (18). Furthermore, in vitro studies have observed that several Y115 mutants of HIV-1 RT displayed reductions in the affinity for the correct dNTP, slight changes in processivity as well as for alterations in the frequency of misinsertions and mispair extensions when compared to wild-type HIV-1 RT_(16, 33, 34). Therefore, alterations in fidelity exhibited by the F155W mutant of MLV RT (Table 1) may also be attributed to decreases in affinity for the correct dNTP, increases in affinity for incorrect dNTP, and/or alterations in processivity as observed with the Y115W mutant of HIV-1 RT (33). In addition, the reduction of RT activity associated with the F155 mutants, including F155W (Table 4), may be due to decrease in the affinity for substrate (dNTP) that may have drastically impeded catalysis. This was proposed for mutants containing substitutions at the homologous position in HIV-1 RT (34). Decreased RT activity may thus account for the drastic reduction in viral titers exhibited by these particular mutants (Tables 1 and 4). In addition, the aromatic side chain of the MLV RT homolog (F155) was proposed and later shown to play a role in the selection of dNTPs over rNTPs $(10,11)$. Our results also support the importance of a bulky side chain at this position because only the F155W and F155Y mutants generated detectable virus titers (Table 1). Recently, the F155V mutant of MLV RT was observed to incorporate UTP (10). Although the F155V mutant in our study exhibited an 18-fold reduction in RT activity (similar to the A154S mutant, characterized by detectable viral replication), the incorporation of rNTPs into the proviral DNA in vivo may account for the reductions in titer greater than 10,000-fold (Table 4). In addition, due to the proximity of amino acids 154 and 155 to each other, the slight 
decrease in fidelity exhibited by the A154S mutant relative to wild type may be attributed to indirect effects exerted on the F155 residue by the alanine to serine substitution.

The other three residues in HIV-1 RT that come in direct contact with the incoming dTTP substrate are residues K65, R72, and Q151 (18). In addition, several R72 and Q151 mutants of HIV-1 RT were characterized by drastic reductions in $\mathrm{K}_{\mathrm{cat}}$ as well as decreased processivity and nucleotidyltransferase activities $(24,39,40)$. Furthermore, similar findings have been made with the R110 residue of MLV RT, which is equivalent to the R72 residue in HIV-1 RT (7). As expected, the majority of substitutions introduced at the homologous positions in MLV RT (K103, R110, and Q190) were characterized by defects in viral replication (Tables 1 and 4), in support of the critical roles of these residues $(7,16,27,39-41)$. A number of HIV-1 RT mutants, such as K65A and Q151N, were previously observed to be more accurate than the wild-type HIV-1 RT in vitro, despite their unaltered affinities toward the dNTP substrates (16). Interestingly, the Q190M mutant of MLV RT exhibited no substantial changes in fidelity, viral replication, or RT activity (Tables 1 and 4), in agreement with previously published results obtained with the equivalent Q151M mutant of HIV-1 RT (16).

In this study, several other positions in MLV RT that are in proximity to the dNTP binding residues were mutated and the effects of these mutations on replication, RT activity, and fidelity were assessed and compared with those of the wild-type MLV RT (Tables 2 to 4). The side chain of F156 in MLV RT (equivalent to F116 in HIV-1 RT) is believed to form a part of the pocket that accommodates the $3^{\prime} \mathrm{OH}$ of the dNTP substrate (18). In addition, analysis of MLV RT crystal structure suggests that the F156 side chain might also be important for correct positioning of the Q190 side chain so that it 
can properly contact the dNTP substrate (11). Our results indicate that the size of the side chain introduced at F156 can affect fidelity, viral replication, and RT activity (Tables 2 and 4). Small side chains (F156I, F156Q, and F156V) exhibited no detectable levels of viral titer, whereas mutants possessing larger side chains (F156L, F156M, F156W, and F156Y) permitted viral replication (Table 2). It is likely that substitutions with amino acids possessing smaller side chains disrupt interactions between residues 156 and 190 in MLV RT, leading to a defect in reverse transcription. Side chains of intermediate sizes (F156L and F156M) may preserve the F156 and Q190 interaction, but alter the local geometry of the dNTP-binding site in a manner that affects the proper function of RT and alters fidelity.

Finally, additional mutations were introduced in MLV RT at various positions located near residues constituting the dNTP-binding site as well as the F156 residue (residues 147 to 152 and 157 to 161). As expected from previous analyses of the D110 position of HIV-1 RT, which is involved in the coordination of the metal cation, the equivalent D150 residue of MLV RT was highly conserved and did not tolerate the D to E or Q substitutions (Table 3) (11, 31, 32). Substitutions of the V148 and L149 positions also resulted in undetectable viral titers, perhaps because they indirectly affected the metal coordination by D150. It is interesting to note that the $\mathrm{L} 151$ residue, which is Cterminal to D150, is more tolerant of substitutions, characterized by the L151F mutant that exhibited a large decrease in fidelity (Table 3).

Although, only small alterations in fidelity (no greater than 3-fold) were observed with the MLV RT mutants of this study in comparison to the wild-type RT, it is possible that the large changes in a specific type of mutation may be altered to a greater extent. 
For instance, a large increase in the rate of a specific substitution ( 1 of the possible 12) may nevertheless be characterized by less than a 3 -fold increase in the overall mutation rate. In addition, an increase in the rate at which deletions occur may account for the large drops in titers associated with many of the MLV RT mutants. Therefore, it will be of interest to see what type of mutations these various RTs generate in comparison to wild type. Finally, retroviral polymerases may be functioning at an optimum, maximizing a number of properties of the RT such as polymerization, processivity, and fidelity. Therefore mutations introduced in RT may have drastic effects in activity as well as fidelity. This may also account for the severe drops in viral titers associated with a number of these MLV RT mutants. Furthermore, this may explain in our study the absence of any mutant possessing a large increase or decrease in the fidelity. Nevertheless, a 3-fold change in the fidelity of these mutants is unlikely to have a large impact on retroviral evolution since selective forces may play a more of an important role as explained by a previously proposed model (8).

The results presented in this study provide insight into the mechanisms by which the fidelity of MLV RT can be manifested through interactions between amino acids and the dNTP substrate. Based on results from previous reports as well as sequence comparison and alignment of MLV RT and HIV-1 RT sequences, we believe that we have mutated the residues constituting the dNTP-binding site of MLV RT. As illustrated in this study, many non-conservative substitutions had deleterious effects on RT activity and viral replication due to the conserved nature of each of these amino acid positions. In contrast, conservative changes reflected properties similar to those of the wild-type RT, especially in the case of fidelity. Therefore, future studies should probably involve the 
residues in MLV RT that are in close proximity to those believed to contact the dNTP substrate. These types of changes will preserve the nature of conserved residues contacting the substrate, yet allow for the positional effects associated with these substitutions to alter the properties of RT such as catalytic activity, fidelity, and processivity. This approach might yield a better understanding of enzyme-substrate interactions and how drug-resistance develops, which may aid in the design of new antiviral drugs.

\section{ACKNOWLEDGMENTS}

We thank Benjamin Beasley, Sara Cheslock, Que Dang, Krista Delviks, WeiShau Hu, Carey Hwang, Timur Kabdulov, Terence Rhodes, Yegor Voronin, and WenHui Zhang for their critical reading of this manuscript and discussion of results. We also thank Erin White and Ronald Mudry, Jr., for their technical support in generating pRMBNB and several of the dNTP-binding site mutants. We especially thank Wei-Shau $\mathrm{Hu}$ for valuable intellectual input and discussions throughout this project. Additionally, we thank Steve Hughes for his intellectual input and discussion. Finally, we extend our thanks to Ann Arthur for her editorial expertise and revisions.

This work was supported by the Public Health Service grant CA58875 from the National Institutes of Health and by the HIV Drug Resistance Program, National Cancer Institute. 
TABLE 1. Effects of mutations in dNTP-binding site residues of MLV RT on the frequency of lac $Z$ inactivation

\begin{tabular}{|c|c|c|c|c|c|}
\hline $\begin{array}{l}\text { MLV RT } \\
\text { genotype }\end{array}$ & $\begin{array}{l}\text { No. } \\
\text { of } \\
\text { expts. }\end{array}$ & $\begin{array}{l}\text { No. of mutant } \\
\text { colonies/ } \\
\text { total colonies }\end{array}$ & $\begin{array}{c}\text { Frequency } \\
\text { of lacZ } \\
\text { inactivation } \\
\text { (mean } \% \pm \\
\text { SE) }\end{array}$ & $\begin{array}{c}\text { Relative } \\
\text { change in } \\
\text { inactivation } \\
\text { of lacZ }\end{array}$ & $\begin{array}{l}\text { Relative } \\
\text { viral titer }\end{array}$ \\
\hline Wildtype & 13 & $522 / 10,300$ & $5.4 \pm 0.23$ & 1.0 & 1.0 \\
\hline \multicolumn{6}{|l|}{ Mutant } \\
\hline K103R & 2 & $154 / 2,740$ & $5.6 \pm 0.35$ & No change & 0.04 \\
\hline K103L & 2 & $\mathrm{NC}^{\mathrm{e}}$ & $\mathrm{NC}$ & $N A^{f}$ & \\
\hline K103S & 2 & $\mathrm{NC}$ & $\mathrm{NC}$ & NA & $<0.0001$ \\
\hline K103W & 2 & $\mathrm{NC}$ & $\mathrm{NC}$ & NA & \\
\hline R110C & 2 & $\mathrm{NC}$ & $\mathrm{NC}$ & NA & \\
\hline R110H & 2 & $\mathrm{NC}$ & $\mathrm{NC}$ & NA & \\
\hline R110I & 2 & $\mathrm{NC}$ & $\mathrm{NC}$ & NA & \\
\hline R110L & 2 & $\mathrm{NC}$ & $\mathrm{NC}$ & NA & $<0.0001$ \\
\hline R110M & 2 & $\mathrm{NC}$ & $\mathrm{NC}$ & NA & \\
\hline R110S & 2 & $\mathrm{NC}$ & $\mathrm{NC}$ & NA & \\
\hline R110T & 2 & $\mathrm{NC}$ & $\mathrm{NC}$ & NA & \\
\hline D153A & 4 & $154 / 1,816$ & $8.4 \pm 0.62$ & 1.6 & 0.0007 \\
\hline D153C & 2 & $65 / 1,154$ & $5.7 \pm 0.23$ & No change & 0.0007 \\
\hline D153Q & 2 & $58 / 1,150$ & $5.2 \pm 0.15$ & No change & 0.001 \\
\hline D153S & 2 & $111 / 2,241$ & $5.1 \pm 0.15$ & No change & 0.03 \\
\hline D153F & 2 & NC & NC & NA & $<0.0001$ \\
\hline D153R & 2 & & & NA & \\
\hline A154S & 2 & $208 / 2,963$ & $7.0 \pm 0.10$ & 1.3 & 0.015 \\
\hline A154E & 2 & NC & NC & NA & \\
\hline A154F & 2 & $\mathrm{NC}$ & $\mathrm{NC}$ & NA & \\
\hline A154L & 2 & $\mathrm{NC}$ & $\mathrm{NC}$ & NA & $<0.0001$ \\
\hline A154N & 2 & $\mathrm{NC}$ & $\mathrm{NC}$ & NA & \\
\hline A154P & 2 & $\mathrm{NC}$ & $\mathrm{NC}$ & NA & \\
\hline A154R & 2 & $\mathrm{NC}$ & $\mathrm{NC}$ & NA & \\
\hline F155W & 2 & $27 / 182$ & $15.1 \pm 1.44$ & 2.8 & 0.0004 \\
\hline F155Y & 2 & $131 / 2,474$ & $5.3 \pm 0.55$ & No change & 0.31 \\
\hline F155D & 2 & $\mathrm{NC}$ & NC & NA & \\
\hline $\mathrm{F} 155 \mathrm{E}$ & 2 & $\mathrm{NC}$ & $\mathrm{NC}$ & NA & \\
\hline F155I & 2 & $\mathrm{NC}$ & $\mathrm{NC}$ & NA & $<0.0001$ \\
\hline F155M & 2 & $\mathrm{NC}$ & $\mathrm{NC}$ & NA & \\
\hline
\end{tabular}


F155V

2

$\mathrm{NC}$

NC

NA

\begin{tabular}{llcccc} 
Q190M & 2 & $44 / 836$ & $5.3 \pm 0.40$ & No change & 0.6 \\
Q190E & 2 & NC & NC & NA & \\
Q190H & 2 & NC & NC & NA & $<0.0001$ \\
Q190N & 2 & NC & NC & NA & \\
\hline
\end{tabular}

${ }^{\mathrm{a}}$ Number of mutant colonies that displayed a white colony phenotype and total number of colonies that were observed in 2 to 13 independent experiments.

${ }^{\mathrm{b}}$ The frequency of lacZ inactivation was calculated as follows: (Number of mutant colonies in each experiment $\div$ by total number of colonies $) \times 100 \%$. The standard errors were determined by using the Two-Sample T-Test.

${ }^{c}$ The relative change in the frequency at which the lac $Z$ gene was inactivated was calculated as follows: frequency of lacZ inactivation observed with mutant MLV RT $\div$ by frequency of lacZ inactivation observed with the wild-type MLV RT. Statistical analysis using a Two-sample T-Test showed that the D153A, A154S, and F155W mutants of MLV RT displayed mutant frequencies significantly higher than that for the wild-type MLV RT $(P<0.03)$. The mutant frequencies obtained with the K103R, D153C, D153Q, D153S, F155Y, and Q190M mutants of MLV RT were not significantly different from that of the wild-type MLV RT $(P>0.05)$.

${ }^{\mathrm{d}}$ Virus titer for each experimental group was determined by serial dilutions and infections. The average virus titer obtained with the wild-type MLV RT for this set of experiments was $9.8 \times 10^{4} \mathrm{CFU} / \mathrm{ml}$. The relative virus titer represents a ratio of the virus titer of mutant MLV RT obtained in each experiment divided by the virus titer obtained with the wild-type MLV RT in parallel experiments.

${ }^{\mathrm{e}} \mathrm{NC}$, represents no colonies present after G418 selection, signifying reductions in viral titers $>$ 10,000-fold. 
${ }^{\mathrm{f}} \mathrm{NA}$, represents not applicable because the relative change in the inactivation of the lac $Z$ gene could not be determined, due to the absence of colonies after G418 selection. 
TABLE 2. Effects of F156 mutations in MLV RT on the frequency of lacZ inactivation

\begin{tabular}{|c|c|c|c|c|c|}
\hline $\begin{array}{l}\text { MLV RT } \\
\text { genotype }\end{array}$ & $\begin{array}{c}\text { No. } \\
\text { of } \\
\text { expts. }\end{array}$ & $\begin{array}{l}\text { No. of mutant } \\
\text { colonies/ } \\
\text { total colonies }\end{array}$ & $\begin{array}{l}\text { Frequency } \\
\text { of lacZ } \\
\text { inactivation } \\
\text { (mean } \% \pm \\
\text { SE) }{ }^{b}\end{array}$ & $\begin{array}{c}\text { Relative } \\
\text { change in } \\
\text { inactivation of } \\
\text { lac }^{\mathrm{c}}\end{array}$ & $\begin{array}{l}\text { Relative } \\
\text { viral titer }\end{array}$ \\
\hline Wild type & 8 & $283 / 5,524$ & $5.5 \pm 0.24$ & 1.0 & 1.0 \\
\hline \multicolumn{6}{|l|}{ Mutant } \\
\hline F156L & 2 & $85 / 955$ & $8.9 \pm 0.20$ & 1.6 & 0.006 \\
\hline F156M & 1 & $20 / 208$ & $9.6 \pm 0.00$ & 1.7 & 0.006 \\
\hline F156W & 2 & $57 / 1,340$ & $4.3 \pm 0.15$ & 0.8 & 0.016 \\
\hline F156Y & 4 & $268 / 6,391$ & $4.3 \pm 0.27$ & 0.8 & 0.046 \\
\hline F156I & 2 & $\mathrm{NC}^{\mathrm{e}}$ & $\mathrm{NC}$ & $N_{A}^{f}$ & \\
\hline F156Q & 2 & $\mathrm{NC}$ & $\mathrm{NC}$ & NA & $<0.0001$ \\
\hline F156V & 2 & $\mathrm{NC}$ & $\mathrm{NC}$ & NA & \\
\hline
\end{tabular}

${ }^{\mathrm{a}}$ The number of mutant colonies that displayed a white colony phenotype and the total number of colonies that were observed in 1 to 8 independent experiments.

${ }^{\mathrm{b}}$ The frequency of $l a c Z$ inactivation was calculated according to Table 1.

c The relative change in the frequency at which the lac $Z$ gene was inactivated was calculated as described in Table 1. Statistical analysis using a Two-Sample T-Test showed that the F156L and F156M mutants of MLV RT displayed mutant frequencies significantly higher than that for the wild-type MLV RT $(P<0.001)$. The F156Y and F156W mutants exhibited mutant frequencies less than and statistically different from that of the wild-type MLV RT $(P<0.05)$.

${ }^{\mathrm{d}}$ Virus titer for each experimental group was determined by serial dilutions and infections. The average virus titer obtained with wild-type MLV RT in this set of experiments was $8.1 \times 10^{4} \mathrm{CFU} / \mathrm{ml}$. The relative virus titer represents a ratio of the virus titer of mutant MLV RT obtained in each experiment divided by the virus titer obtained with the wildtype MLV RT in parallel experiments. 
${ }^{\mathrm{e}} \mathrm{NC}$, represents no colonies present after G418 selection, signifying reductions in viral titers $>10,000$-fold.

${ }^{\mathrm{f}} \mathrm{NA}$, represents not applicable, since the relative change in the inactivation of the lac Z gene could not be determined, due to the absence of colonies after G418 selection. 
TABLE 3. Effects of mutations in residues proximal to residues of the dNTP-binding site of MLV RT on the frequency of lacZ inactivation

\begin{tabular}{cccccc}
\hline $\begin{array}{c}\text { MLV RT } \\
\text { genotype }\end{array}$ & $\begin{array}{c}\text { No. } \\
\text { of } \\
\text { expts. }\end{array}$ & $\begin{array}{c}\text { No. of mutant } \\
\text { colonies/ } \\
\text { total colonies }^{\mathrm{a}}\end{array}$ & $\begin{array}{c}\text { Frequency of } \\
\text { lacZ } \\
\text { inactivation } \\
(\text { mean } \% \pm \\
\text { SE) }\end{array}$ & $\begin{array}{c}\text { Relative change } \\
\text { in inactivation } \\
\text { of lacZ }^{\mathrm{b}}\end{array}$ & $\begin{array}{c}\text { Relative } \\
\text { viral titer }^{\mathrm{d}}\end{array}$ \\
\hline Wild type & 6 & $271 / 5,220$ & $5.3 \pm 0.26$ & 1.0 & 1.0 \\
Mutant & & & & & \\
T147A & 2 & $66 / 969$ & $6.8 \pm 0.10$ & 1.3 & 0.02 \\
V148D & 2 & $\mathrm{NC}$ & $\mathrm{NC}$ & $\mathrm{NA}^{\mathrm{f}}$ & $<0.0001$ \\
L149W & 2 & $\mathrm{NC}$ & $\mathrm{NC}$ & $\mathrm{NA}$ & $<0.0001$ \\
D150E & 2 & $\mathrm{NC}$ & $\mathrm{NC}$ & $\mathrm{NA}$ & $<0.0001$ \\
L151F & 2 & $413 / 3,304$ & $12.5 \pm 0.50$ & 2.4 & 0.12 \\
K152A & 2 & $109 / 1,438$ & $7.6 \pm 0.65$ & 1.4 & 0.71 \\
C157A & 2 & $69 / 1,031$ & $6.8 \pm 0.20$ & 1.3 & 1.10 \\
R159A & 2 & $51 / 797$ & $6.3 \pm 0.35$ & No change & 0.51 \\
H161A & 2 & $139 / 2,202$ & $6.3 \pm 0.20$ & 1.2 & 0.45 \\
\hline
\end{tabular}

${ }^{\mathrm{a}}$ The number of mutant colonies that displayed a white colony phenotype and total number of colonies that were observed in 2 to 6 independent experiments.

${ }^{\mathrm{b}}$ The frequency of lacZ inactivation was calculated according to Table 1.

${ }^{c}$ The relative change in the frequency at which the lac $Z$ gene was inactivated was calculated as for in Table 1. Statistical analysis using a Two-Sample T-Test showed that the T147A, L151F, K152A, C157A, and H161A mutants of MLV RT displayed mutant frequencies significantly higher than that of the wild-type MLV RT $(P<0.04)$. The mutant frequency obtained with the R159A mutant of MLV RT was not significantly different from that of the wild-type MLV RT $(P>0.09)$.

${ }^{\mathrm{d}}$ Virus titer for each experimental group was determined by serial dilutions and infections. The average virus titer obtained with the wild-type MLV RT in this set of experiments was $3.6 \times 10^{4} \mathrm{CFU} / \mathrm{ml}$. The relative virus titer represents a ratio of the virus 
titer of mutant MLV RT obtained in each experiment divided by the virus titer obtained with the wild-type MLV RT in parallel experiments.

${ }^{\mathrm{e}} \mathrm{NC}$, represents no colonies present after G418 selection, signifying reductions in viral titers $>$ 10,000-fold.

${ }^{\mathrm{f}} \mathrm{NA}$, represents not applicable, since the relative change in the inactivation of the lacZ gene could not be determined, due to the absence of colonies after G418 selection. 
TABLE 4. RT activities of dNTP-binding site mutants of MLV RT.

\begin{tabular}{|c|c|c|}
\hline $\begin{array}{l}\text { MLV RT } \\
\text { genotype }\end{array}$ & $\begin{array}{c}\text { Average RT activity as (\%) of wild } \\
\text { type }^{\mathrm{a}} \\
\text { (mean of } \% \pm \mathrm{SE} \text { ) }\end{array}$ & Viral titer as a (\%) of wild type ${ }^{b}$ \\
\hline Wild type & 100 & 100 \\
\hline T147A & $15.4 \pm 0.9$ & 2.0 \\
\hline V148D & $3.0 \pm 0.9$ & $<0.01$ \\
\hline L149W & $1.0 \pm 0.3$ & $<0.01$ \\
\hline D150E & $1.3 \pm 0.4$ & $<0.01$ \\
\hline $\mathrm{L} 151 \mathrm{~F}$ & $86.7 \pm 0.4$ & 12.0 \\
\hline K152A & $37.9 \pm 15$ & 71.0 \\
\hline K103L & $0.9 \pm 0.5$ & $<0.01$ \\
\hline K103R & $15.6 \pm 4.5$ & 4.0 \\
\hline K103W & $0.5 \pm 0.2$ & $<0.01$ \\
\hline R110C & $0.8 \pm 0.6$ & $<0.01$ \\
\hline R110H & $0.9 \pm 0.6$ & $<0.01$ \\
\hline R110I & $0.5 \pm 0.1$ & $<0.01$ \\
\hline R110L & $0.2 \pm 0.1$ & $<0.01$ \\
\hline R110M & $0.3 \pm 0.0$ & $<0.01$ \\
\hline R110S & $0.3 \pm 0.1$ & $<0.01$ \\
\hline R110T & $0.4 \pm 0.1$ & $<0.01$ \\
\hline D153A & $6.7 \pm 1.0$ & 0.04 \\
\hline D153C & $9.9 \pm 2.8$ & 0.07 \\
\hline D153F & $2.4 \pm 0.3$ & $<0.01$ \\
\hline D153Q & $8.0 \pm 0.6$ & 0.1 \\
\hline D153R & $1.3 \pm 0.7$ & $<0.01$ \\
\hline D153S & $23 . \pm 4.3$ & 3.0 \\
\hline A154E & $0.3 \pm 0.1$ & $<0.01$ \\
\hline A154F & $0.2 \pm 0.0$ & $<0.01$ \\
\hline A154L & $0.4 \pm 0.0$ & $<0.01$ \\
\hline A154N & $0.2 \pm 0.1$ & $<0.01$ \\
\hline A154P & $0.3 \pm 0.1$ & $<0.01$ \\
\hline A154R & $0.4 \pm 0.2$ & $<0.01$ \\
\hline A154S & $5.5 \pm 0.9$ & 2.0 \\
\hline F155D & $0.2 \pm 0.2$ & $<0.01$ \\
\hline $\mathrm{F} 155 \mathrm{E}$ & $0.9 \pm 0.1$ & $<0.01$ \\
\hline F155I & $4.9 \pm 3.6$ & $<0.01$ \\
\hline
\end{tabular}




$\begin{array}{ccc}\text { F155M } & 2.0 \pm 0.4 & <0.01 \\ \text { F155V } & 5.5 \pm 2.6 & <0.01 \\ \text { F155W } & 8.0 \pm 3.5 & 4.0 \\ \text { F155Y } & 161.4 \pm 36.9 & 31.0 \\ & & <0.01 \\ \text { F156I } & 0.8 \pm 0.4 & 0.6 \\ \text { F156L } & 2.4 \pm 1.3 & 0.6 \\ \text { F156M } & 8.5 \pm 3.1 & <0.01 \\ \text { F156Q } & 1.1 \pm 0.5 & <0.01 \\ \text { F156V } & 1.5 \pm 0.3 & 1.6 \\ \text { F156W } & 11.1 \pm 0.9 & 3.0 \\ \text { F156Y } & 14.8 \pm 1.2 & 110 \\ \text { C157A } & & 50.0 \\ \text { R159A } & 56.1 \pm 6.0 & 130.0 \\ \text { H161A } & 30.6 \pm 6.5 & <0.01 \\ \text { Q190E } & 43.6 \pm 19.1 & <0.01 \\ \text { Q190H } & 1.0 \pm 0.4 & 60.0 \\ \text { Q190M } & 0.3 \pm 0.1 & <0.01 \\ \text { Q190N } & 51.6 \pm 2.2 & \end{array}$

${ }^{\text {a }}$ Average RT activities using virion-associated RT. The activities shown are relative to the wild-type MLV RT (set to 100\%) using the Poly (rA)-Oligo d (T) template-primer complex. The results represent an average of 2 to 3 experiments. SE represents the standard error of the mean. RT activities of all mutants seem to be statistically different from that of the wild-type MLV RT $(P<0.002)$, except for the L151F, K152A, F155Y, C157A and H161A mutants $(P>0.05)$.

${ }^{\mathrm{b}}$ Viral titers of mutants as a (\%) of the wild-type titer (set at 100\%). Viral titers represent the ratio of the virus titer generated from mutant MLV RT obtained in each experiment divided by the virus titer generated from the wild-type MLV RT in parallel experiments and multiplied by 100 . 
FIG. 1. Structures of MLV-based constructs and rapid in vivo assay to identify structural determinants in MLV RT that are important for fidelity. The ANGIE P encapsidating cell line is a D17-based cell line expressing both the MLV-based vector pGA-1 and pSVA-MLV-env. The pGA-1 vector, which contains long terminal repeats (LTRs) and all cis-acting elements of MLV, transcribes the E. coli lacZ and neo genes from the LTR promoter. The internal ribosomal entry site (IRES) of encephalomyocarditis virus is used to express neo. $\mathrm{pSV}-\mathrm{A}-\mathrm{MLV}$-env expresses the amphotropic MLV envelope from a truncated MLV LTR ( $\triangle \mathrm{LTR})$ and the SV40 promoter-enhancer. (1) Transfection of the wild-type or mutated MLV gag/pol construct pLGPS into the ANGIE P cell line allows for expression of the MLV gag and pol from the $\Delta \mathrm{LTR}$. Infectious viral particles are (2) harvested and (3) used to infect D17 cells. G418 ${ }^{\mathrm{R}}$ infected colonies are (4) selected, resistant clones are (5) stained with $\mathrm{X}-\mathrm{Gal}$, and (6) the frequency at which lacZ is inactivated is determined. 


\section{Experimental Protocol}

pLGPS

\begin{tabular}{|c|c|c|c|}
\hline$\Delta \mathrm{LTR}$ & gag & pol & $\mathbf{p}$ \\
\hline
\end{tabular}

$\downarrow$ 1. Transfection

ANGIE P Cell Line

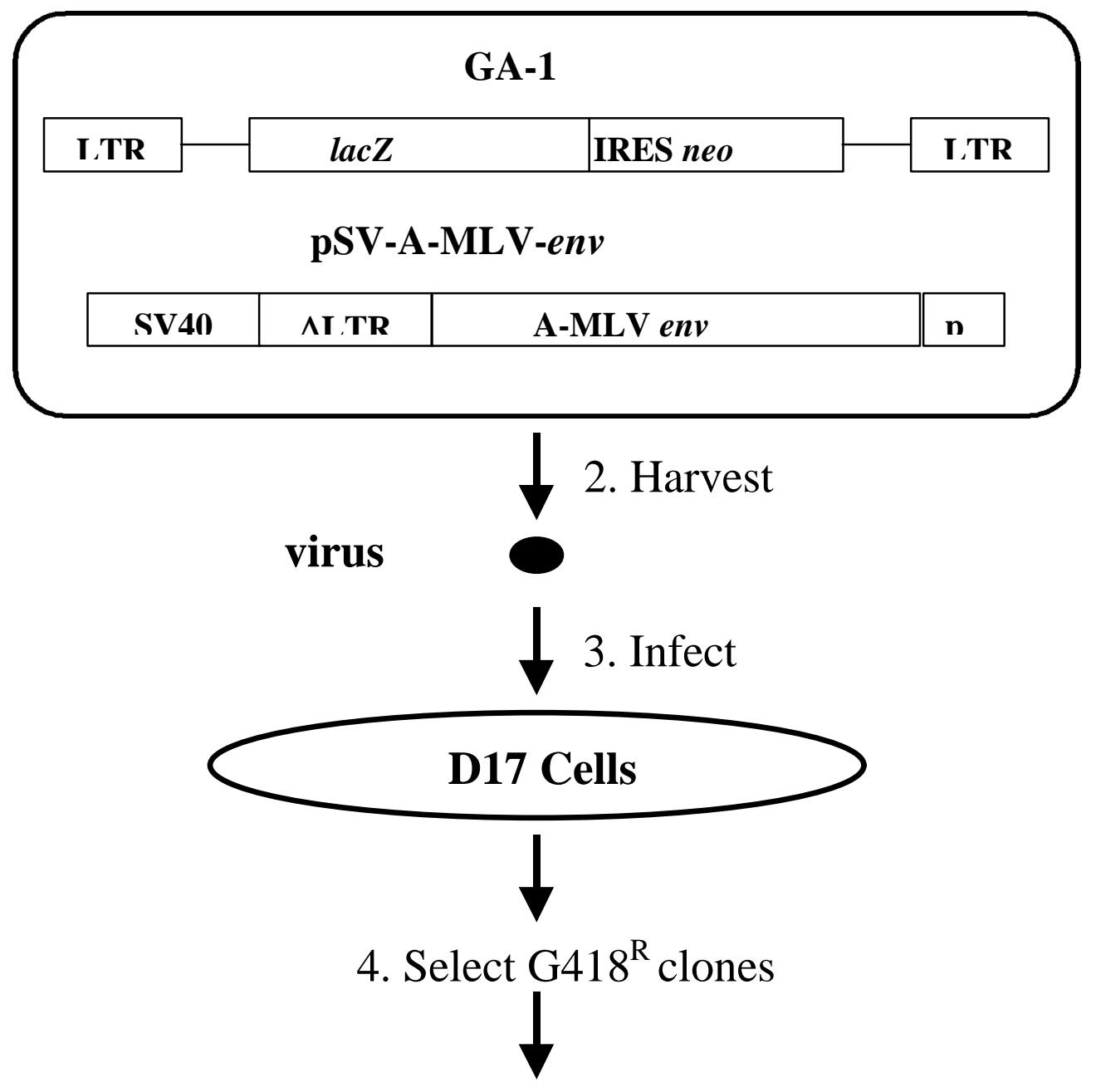

5. Stain w/ X-Gal

$\downarrow$

6. Determine Frequency of

lacZ inactivation 
FIG. 2. The dNTP-binding sites of MLV and HIV-1 RTs. (A) Primary sequence homology of the MLV and HIV-1 RT dNTP-binding sites. HIV-1 and MLV RTs were aligned through their TVLD, LPQG, and YXDD amino acid motifs. Residues of the dNTP-binding site of MLV RT were determined by further alignment with residues proposed to constitute the dNTP-binding site of HIV-1 RT. Two dots represent residues that are identical, whereas one dot represents conservative changes between the two RTs. Bold lettering represents residues constituting the dNTP-binding sites in both HIV-1 and MLV RTs. (B) Structure of HIV-1 RT dNTP-binding site modified from Huang et al. (16). The restricted structure contains amino acid positions K65, R72, D113, A114, Y115, and Q151 interacting with the dTTP substrate. Italic lettering represents the equivalent residues in MLV RT constituting the dNTP-binding site. Components of the dTTP substrate are denoted as B, base; R, ribose sugar; and P, phosphate. Dashed lines represent interactions between the amino acid residues of RT and the dTTP substrate; mc represents main chain interactions between the substrate and the RT. (C) Comparison of secondary structures of MLV and HIV-1 RTs constituting the dNTP-binding sites. The backbone of HIV-1 RT (amino acid sequences 63 to 76, 106 to 121, and 148 to 154) was superimposed on the homologous structure in MLV RT (amino acid sequences 101 to 114, 146 to 161, and 187 to 193). HIV-1 RT is represented in gray; MLV RT, in black. Side chains of residues creating the dNTP-binding sites of MLV and HIV-1 RTs are illustrated as wire frames. 


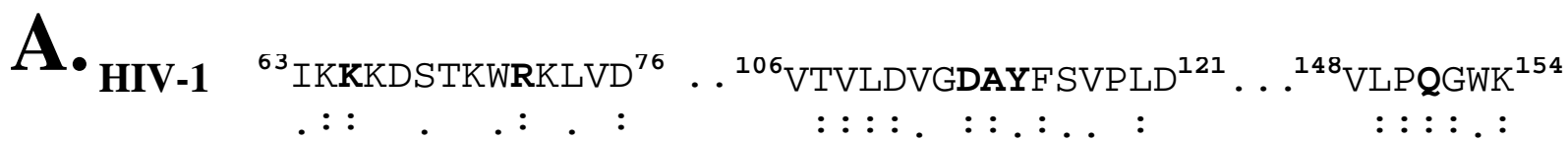
MLV $\begin{gathered}{ }^{101} \text { VKKPGTNDYRPVQD } \\ { }^{114} \ldots{ }^{146}{ }^{14 T V L D L K D A F F C L R L H}{ }^{161} \ldots\end{gathered}$

B.

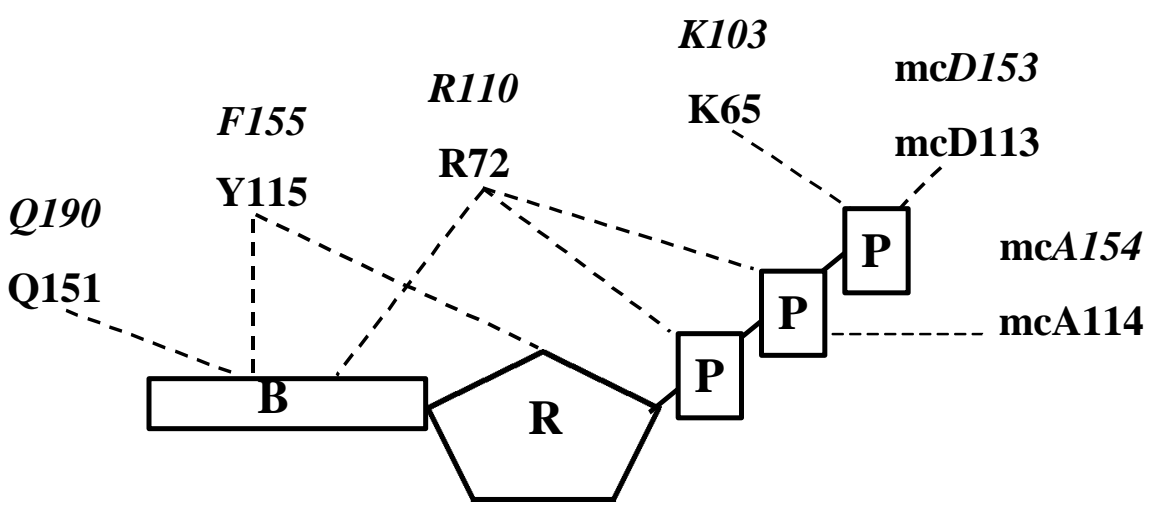

C.

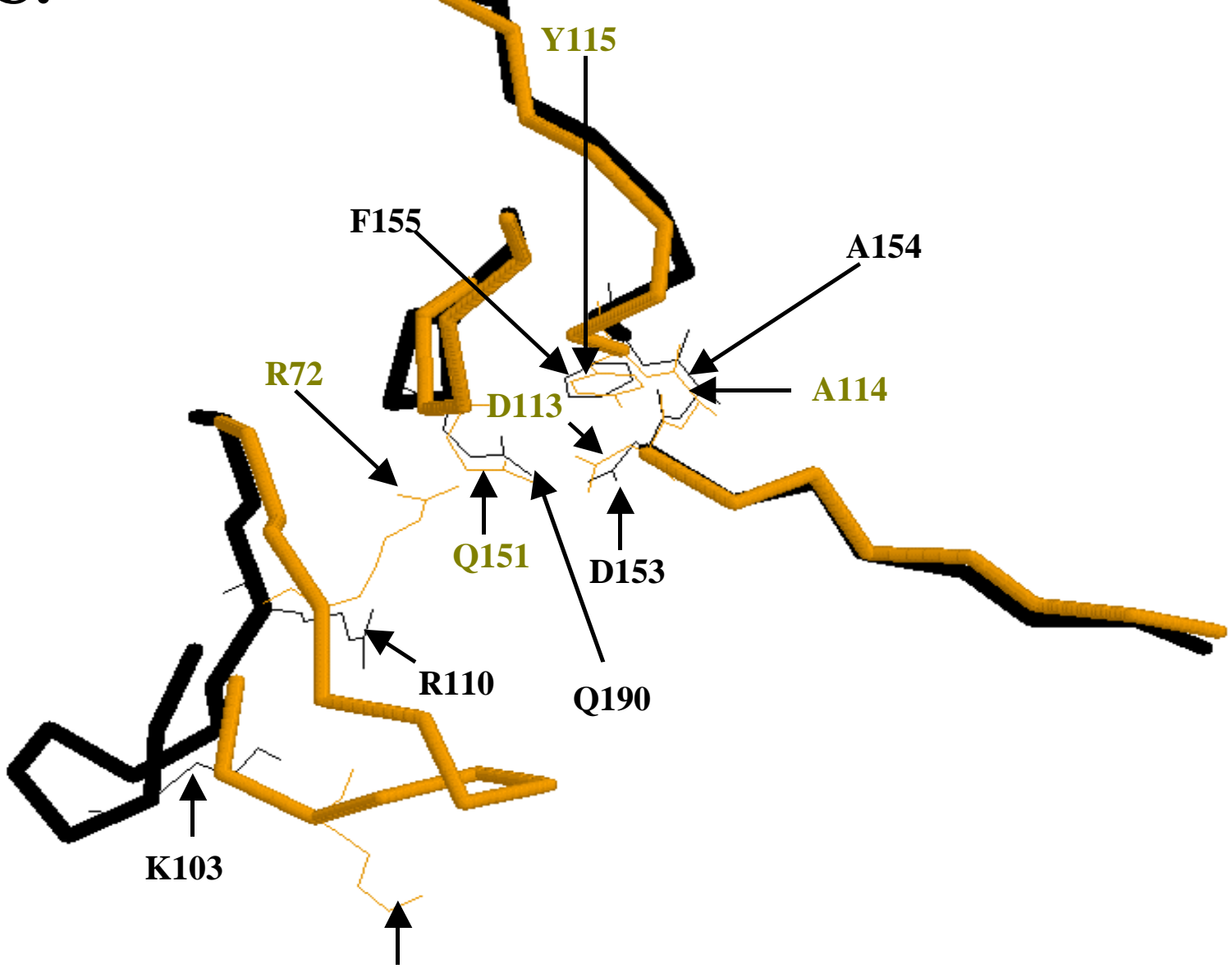

K65 


\section{REFERENCES}

1. Bakhanashvili, M., O. Avidan, and A. Hizi. 1996. Mutational studies of human immunodeficiency virus type 1 reverse transcriptase: the involvement of residues 183 and 184 in the fidelity of DNA synthesis. FEBS Lett. 391:257-262.

2. Basu, A., V. B. Nanduri, G. F. Gerard, and M. J. Modak. 1988. Substrate binding domain of murine leukemia virus reverse transcriptase. Identification of lysine 103 and lysine 421 as binding site residues. J. Biol. Chem. 263:1648-1653.

3. Beard, W. A., K. Bebenek, T. A. Darden, L. Li, R. Prasad, T. A. Kunkel, and S. H. Wilson. 1998. Vertical-scanning mutagenesis of a critical tryptophan in the minor groove binding track of HIV-1 reverse transcriptase. Molecular nature of polymerase-nucleic acid interactions. J. Biol. Chem. 273:30435-30442.

4. Bebenek, K., W. A. Beard, J. R. Casas-Finet, H. R. Kim, T. A. Darden, S. H. Wilson, and T. A. Kunkel. 1995. Reduced frameshift fidelity and processivity of HIV-1 reverse transcriptase mutants containing alanine substitutions in helix $\mathrm{H}$ of the thumb subdomain. J. Biol. Chem. 270:19516-19523.

5. Borman, A. M., C. Quillent, P. Charneau, K. M. Kean, and F. Clavel. 1995. A highly defective HIV-1 group O provirus: evidence for the role of local sequence determinants in G-->A hypermutation during negative- strand viral DNA synthesis. Virology. 208:601-609.

6. Chesebro, B., W. Britt, L. Evans, K. Wehrly, J. Nishio, and M. Cloyd. 1983. Characterization of monoclonal antibodies reactive with murine leukemia viruses: use in analysis of strains of friend MCF and Friend ecotropic murine leukemia virus. Virology. 127:134-148. 
7. Chowdhury, K., N. Kaushik, V. N. Pandey, and M. J. Modak. 1996. Elucidation of the role of Arg 110 of murine leukemia virus reverse transcriptase in the catalytic mechanism: biochemical characterization of its mutant enzymes. Biochemistry. 35:16610-16620.

8. Coffin, J. M. 1995. HIV population dynamics in vivo: implications for genetic variation, pathogenesis, and therapy [see comments]. Science. 267:483-489.

9. Drosopoulos, W. C., and V. R. Prasad. 1996. Increased polymerase fidelity of E89G, a nucleoside analog-resistant variant of human immunodeficiency virus type 1 reverse transcriptase. J. Virol. 70:4834-4838.

10. Gao, G., and S. P. Goff. 1998. Replication defect of moloney murine leukemia virus with a mutant reverse transcriptase that can incorporate ribonucleotides and deoxyribonucleotides. J. Virol. 72:5905-5911.

11. Georgiadis, M. M., S. M. Jessen, C. M. Ogata, A. Telesnitsky, S. P. Goff, and W. A. Hendrickson. 1995. Mechanistic implications from the structure of a catalytic fragment of Moloney murine leukemia virus reverse transcriptase. Structure. 3:879-892.

12. Gu, Z., E. J. Arts, M. A. Parniak, and M. A. Wainberg. 1995. Mutated K65R recombinant reverse transcriptase of human immunodeficiency virus type 1 shows diminished chain termination in the presence of 2',3'-dideoxycytidine 5'triphosphate and other drugs. Proc. Natl. Acad. Sci. USA. 92:2760-2764.

13. Gu, Z., Q. Gao, H. Fang, H. Salomon, M. A. Parniak, E. Goldberg, J. Cameron, and M. A. Wainberg. 1994. Identification of a mutation at codon 65 in the IKKK motif of reverse transcriptase that encodes human immunodeficiency 
virus resistance to 2',3'-dideoxycytidine and 2',3'-dideoxy-3'-thiacytidine. Antimicrob Agents Chemother. 38:275-281.

14. Gutierrez-Rivas, M., A. Ibanez, M. A. Martinez, E. Domingo, and L. Menendez-Arias. 1999. Mutational analysis of Phe160 within the "palm" subdomain of human immunodeficiency virus type 1 reverse transcriptase. J. Mol. Biol. 290:615-625.

15. Halvas, E. K., E. S. Svarovskaia, and V. K. Pathak. 2000. Development of an In vivo assay To identify structural determinants in murine leukemia virus reverse transcriptase important for fidelity [In Process Citation]. J. Virol. 74:312-319.

16. Harris, D., N. Kaushik, P. K. Pandey, P. N. Yadav, and V. N. Pandey. 1998. Functional analysis of amino acid residues constituting the dNTP binding pocket of HIV-1 reverse transcriptase. J. Biol. Chem. 273:33624-33634.

17. Hsiou, Y., J. Ding, K. Das, A. D. Clark, Jr., S. H. Hughes, and E. Arnold. 1996. Structure of unliganded HIV-1 reverse transcriptase at 2.7 A resolution: implications of conformational changes for polymerization and inhibition mechanisms. Structure. 4:853-860.

18. Huang, H., R. Chopra, G. L. Verdine, and S. C. Harrison. 1998. Structure of a covalently trapped catalytic complex of HIV-1 reverse transcriptase: implications for drug resistance [see comments]. Science. 282:1669-1675.

19. Iversen, A. K., R. W. Shafer, K. Wehrly, M. A. Winters, J. I. Mullins, B. Chesebro, and T. C. Merigan. 1996. Multidrug-resistant human immunodeficiency virus type 1 strains resulting from combination antiretroviral therapy. J. Virol. 70:1086-1090. 
20. Jacobo-Molina, A., J. Ding, R. G. Nanni, A. D. Clark, Jr., X. Lu, C. Tantillo, R. L. Williams, G. Kamer, A. L. Ferris, P. Clark, and et al. 1993. Crystal structure of human immunodeficiency virus type 1 reverse transcriptase complexed with double-stranded DNA at 3.0 A resolution shows bent DNA. Proc. Natl. Acad. Sci. USA. 90:6320-6324.

21. Jang, S. K., H. G. Krausslich, M. J. Nicklin, G. M. Duke, A. C. Palmenberg, and E. Wimmer. 1988. A segment of the 5' nontranslated region of encephalomyocarditis virus RNA directs internal entry of ribosomes during in vitro translation. J. Virol. 62:2636-2643.

22. Julias, J. G., D. Hash, and V. K. Pathak. 1995. E- vectors: development of novel self-inactivating and self-activating retroviral vectors for safer gene therapy. J. Virol. 69:6839-6846.

23. Julias, J. G., T. Kim, G. Arnold, and V. K. Pathak. 1997. The antiretrovirus drug 3'-azido-3'-deoxythymidine increases the retrovirus mutation rate. J. Virol. 71:4254-4263.

24. Kaushik, N., D. Harris, N. Rege, M. J. Modak, P. N. Yadav, and V. N. Pandey. 1997. Role of glutamine-151 of human immunodeficiency virus type-1 reverse transcriptase in RNA-directed DNA synthesis. Biochemistry. 36:1443014438.

25. Kent, R. B., J. R. Emanuel, Y. Ben Neriah, R. Levenson, and D. E. Housman. 1987. Ouabain resistance conferred by expression of the cDNA for a murine $\mathrm{Na}+$, K+-ATPase alpha subunit. Science. 237:901-903. 
26. Kim, B., T. R. Hathaway, and L. A. Loeb. 1998. Fidelity of mutant HIV-1 reverse transcriptases: interaction with the single-stranded template influences the accuracy of DNA synthesis. Biochemistry. 37:5831-5839.

27. Kim, B., T. R. Hathaway, and L. A. Loeb. 1996. Human immunodeficiency virus reverse transcriptase. Functional mutants obtained by random mutagenesis coupled with genetic selection in Escherichia coli. J. Biol. Chem. 271:4872-4878.

28. Kim, T., R. A. Mudry, Jr., C. A. Rexrode, 2nd, and V. K. Pathak. 1996. Retroviral mutation rates and A-to-G hypermutations during different stages of retroviral replication. J. Virol. 70:7594-7602.

29. Kohlstaedt, L. A., J. Wang, J. M. Friedman, P. A. Rice, and T. A. Steitz. 1992. Crystal structure at 3.5 A resolution of HIV-1 reverse transcriptase complexed with an inhibitor. Science. 256:1783-1790.

30. Landau, N. R., K. A. Page, and D. R. Littman. 1991. Pseudotyping with human T-cell leukemia virus type I broadens the human immunodeficiency virus host range. J. Virol. 65:162-169.

31. Larder, B. A., D. J. Purifoy, K. L. Powell, and G. Darby. 1987. Site-specific mutagenesis of AIDS virus reverse transcriptase. Nature. 327:716-717.

32. Lowe, D. M., V. Parmar, S. D. Kemp, and B. A. Larder. 1991. Mutational analysis of two conserved sequence motifs in HIV-1 reverse transcriptase. FEBS Lett. 282:231-234.

33. Martin-Hernandez, A. M., E. Domingo, and L. Menendez-Arias. 1996. Human immunodeficiency virus type 1 reverse transcriptase: role of Tyr115 in 
deoxynucleotide binding and misinsertion fidelity of DNA synthesis. EMBO J. 15:4434-4442.

34. Martin-Hernandez, A. M., M. Gutierrez-Rivas, E. Domingo, and L. Menendez-Arias. 1997. Mispair extension fidelity of human immunodeficiency virus type 1 reverse transcriptases with amino acid substitutions affecting Tyr115. Nucleic Acids Res. 25:1383-1389.

35. Miller, A. D., and C. Buttimore. 1986. Redesign of retrovirus packaging cell lines to avoid recombination leading to helper virus production. Mol. Cell. Biol. 6:2895-2902.

36. Poch, O., I. Sauvaget, M. Delarue, and N. Tordo. 1989. Identification of four conserved motifs among the RNA-dependent polymerase encoding elements. EMBO J. 8:3867-3874.

37. Roberts, J. D., K. Bebenek, and T. A. Kunkel. 1988. The accuracy of reverse transcriptase from HIV-1. Science. 242:1171-1173.

38. Sambrook, J., E. F. Fritsch,and T. Maniatis. 1989. Molecular cloning: a laboratory manual, 2nd ed. Cold Spring Harbor Laboratory Press, Cold Spring Harbor, N.Y.

39. Sarafianos, S. G., V. N. Pandey, N. Kaushik, and M. J. Modak. 1995. Glutamine 151 participates in the substrate dNTP binding function of HIV-1 reverse transcriptase. Biochemistry. 34:7207-7216.

40. Sarafianos, S. G., V. N. Pandey, N. Kaushik, and M. J. Modak. 1995. Sitedirected mutagenesis of arginine 72 of HIV-1 reverse transcriptase. Catalytic role and inhibitor sensitivity. J. Biol. Chem. 270:19729-19735. 
41. Tantillo, C., J. Ding, A. Jacobo-Molina, R. G. Nanni, P. L. Boyer, S. H. Hughes, R. Pauwels, K. Andries, P. A. Janssen, and E. Arnold. 1994. Locations of anti-AIDS drug binding sites and resistance mutations in the threedimensional structure of $\mathrm{HIV}-1$ reverse transcriptase. Implications for mechanisms of drug inhibition and resistance. J. Mol. Biol. 243:369-387.

42. Temin, H. M. 1993. Retrovirus variation and reverse transcription: abnormal strand transfers result in retrovirus genetic variation. Proc. Natl. Acad. Sci. USA. 90:6900-6903.

43. Tisdale, M., T. Alnadaf, and D. Cousens. 1997. Combination of mutations in human immunodeficiency virus type 1 reverse transcriptase required for resistance to the carbocyclic nucleoside 1592U89. Antimicrob Agents Chemother. 41:1094-1098.

44. Ueno, T., T. Shirasaka, and H. Mitsuya. 1995. Enzymatic characterization of human immunodeficiency virus type 1 reverse transcriptase resistant to multiple 2',3'-dideoxynucleoside 5'- triphosphates. J. Biol. Chem. 270:23605-23611.

45. Vandamme, A. M., K. Van Vaerenbergh, and E. De Clercq. 1998. Anti-human immunodeficiency virus drug combination strategies. Antivir Chem Chemother. 9:187-203.

46. Vara, J. A., A. Portela, J. Ortin, and A. Jimenez. 1986. Expression in mammalian cells of a gene from Streptomyces alboniger conferring puromycin resistance. Nucleic Acids Res. 14:4617-4624.

47. Wainberg, M. A., W. C. Drosopoulos, H. Salomon, M. Hsu, G. Borkow, M. Parniak, Z. Gu, Q. Song, J. Manne, S. Islam, G. Castriota, and V. R. Prasad. 
1996. Enhanced fidelity of 3TC-selected mutant HIV-1 reverse transcriptase.

Science. 271:1282-1285. 


\title{
CHAPTER 4
}

\section{Wild-Type and YMDD Mutant of Murine Leukemia Virus Reverse Transcriptase Are Resistant to $2^{\prime}, 3^{\prime}$-Dideoxy-3'-thiacytidine}

\author{
Elias K. Halvas, ${ }^{1}$ Evguenia S. Svarovskaia, ${ }^{1,2}$ Eric O. Freed, ${ }^{3}$ and Vinay K. Pathak ${ }^{1,2} *$ \\ Mary Babb Randolph Cancer Center and Department of Biochemistry, West Virginia \\ University, Morgantown, West Virginia 26506, ${ }^{1}$ HIV Drug Resistance Program, National \\ Cancer Institute, FCRDC, Frederick, Maryland 21702-1201, ${ }^{2}$ and Laboratory of \\ Molecular Microbiology, National Institute of Allergy and Infectious Diseases, National \\ Institutes of Health, Bethesda, Maryland 20892-0460 3
}

This manuscript was submitted for publication to the Journal of Virology, February 2000 


\begin{abstract}
The antiretroviral nucleoside analog $2^{\prime}, 3^{\prime}$-dideoxy-3'-thiacytidine (3TC) is a potent inhibitor of wild-type human immunodeficiency virus type 1 (HIV-1) reverse transcriptase (RT). A methionine-to-valine or methionine-to-isoleucine substitution at residue 184 in the HIV-1 YMDD motif, which is located at the $\mathrm{RT}$ active site, leads to a high level of resistance to 3TC. We sought to determine whether 3TC can inhibit the replication of wild-type murine leukemia virus (MLV) RT, which contains V223 at the YVDD active site motif, and of the V223M, V223I, V223A, and V223S mutant RTs. Surprisingly, the wild-type and all four of the V223 mutants of MLV RT were highly resistant to 3TC. These results indicate that determinants outside the YVDD motif of MLV RT confer a high level of resistance to 3TC. Therefore, structural differences among similar RTs might result in widely divergent sensitivities to antiretroviral nucleoside analogs.
\end{abstract}


Currently, 14 antiviral drugs have been approved for clinical use to combat human immunodeficiency virus type 1 (HIV-1) infections $(11,54)$. When used in combination, many of these drugs have been shown to prolong the life expectancy of infected individuals and slow the progression of AIDS (12, 14, 20, 21, 40, 42). Most of these drugs, both nucleoside and nonnucleoside inhibitors, have been designed to target reverse transcriptase $(\mathrm{RT})(2,10,53)$. One of the compounds used in combination therapy is the nucleoside analog $2^{\prime}, 3^{\prime}$-dideoxy-3'-thiacytidine (3TC) $(4,20,21,37,51)$. The triphosphate form of 3TC inhibits reverse transcription through chain termination of DNA polymerization, a function carried out by RT, and therefore has been observed to be a potent inhibitor of both HIV type 1 and type 2 replication $(2,9,10)$. In addition to the clinical benefits $(20,21,37,46)$ associated with the use of this nucleoside analog, 3TC exhibits low toxicity, even at millimolar concentrations $(7,9,15,36)$.

All of the drugs being used for therapy to combat HIV-1 infections eventually result in drug-resistant mutants, thereby allowing for the progression of the disease. Resistance to 3TC is no exception and is characterized by a mutation at the methionine 184 position of the Tyr-Met-Asp-Asp (YMDD) motif found in HIV-1 RT (5, 17, 31, 48, 55). A YXDD motif, where $X$ is a variable amino acid, is highly conserved among the many viral RNA polymerases as well as RNA-dependent DNA polymerases (43). For example, Rous sarcoma virus RT contains the YMDD motif, the RTs of retroelements such as 297 (Gypsy-like group) and Int 32 (Line-like group) contain both the YLDD and YADD sequences, and the poliovirus RNA polymerase contains the YGDD motif (43). Mutations in the YXDD motif abolish enzymatic activity and alter the processivity and fidelity of RT $(3,6,23,27,39)$. The prevalent mutation in HIV-1 RT associated with 
3TC resistance is the M184V substitution $(17,48,55)$, which confers a level of resistance 1000 times greater than that displayed by the wild-type enzyme $(17,55)$. It has been observed that the M184I variant, which is resistant to 3TC but is less catalytically active, is selected first after initiation of 3TC treatment $(5,6,31,55)$, and then is replaced by the M184V variant after long-term treatment with 3TC.

Mutations in the motif analogous to the HIV-1 RT YMDD domain are also correlated with resistance to 3TC in other retroviruses. The YVDD, YIDD, and YTDD motifs are selected during 3TC treatment of cells infected with simian immunodeficiency virus (SIV) or feline immunodeficiency virus (FIV) $(8,49)$. In addition, the YVDD and YIDD motifs arise during 3TC treatment of hepatitis B virus (HBV)-infected cells and patients (1). The selection for mutations in the YXDD motif in other retroviruses as well as in HBV has suggested that this determinant is widely associated with 3TC sensitivity.

Other mutations in HIV-1 and FIV RTs have been implicated to confer dual resistance to 3TC and other nucleoside analogs. Specifically, the E89G and G333E mutations in HIV-1 RT are correlated with dual resistance to $3 \mathrm{TC}$ and either phosphonoformic acid or 3'-azido-3'-deoxythymidine (AZT), respectively, in tissue culture $(29,45)$. The K65R mutation in HIV-1 RT is associated with resistance to 3TC and $2^{\prime}, 3^{\prime}$-dideoxycytidine (ddC) (19). Finally, the P156S mutation in FIV RT appears to confer resistance to both AZT and 3TC (50).

Although HIV-1 and MLV RTs share only 25\% amino acid sequence identity, the two proteins are structurally similar (18). Comparison of the finger and palm domains in HIV-1 and MLV RT crystal structures reveals similar tertiary structures (18, 25, 34). In addition, many of the sequence motifs present in HIV-1 RT, such as the 
YXDD domain, the dNTP-binding site, and the conserved Leu-Pro-Gln-Gly (LPQG) motif, are also present in MLV RT. Importantly, the antiretroviral nucleoside analogs AZT, ddC, 2',3'-dideoxyinosine (ddI), and 2',3'-didehydro-3'-deoxythimidine (d4T), which inhibit HIV-1 RT, also inhibit MLV RT (52). Therefore, since wild-type MLV RT contains the YVDD motif, it was expected and recently shown to be resistant to 3TC (44).

Based on the similarities between the HIV-1 and MLV RTs, we hypothesized that the YMDD mutant of MLV RT would be sensitive to 3TC and the YIDD mutant would be resistant. To test this hypothesis, we generated viruses containing wild-type MLV RT as well as several mutants at position V223 within the YVDD motif and compared the titers of these viruses in several different target cells in the presence or absence of 3TC.

MLV mutants, target cells, and the ANGIE P cell line. The construction of the V223A, V223I, V223M, and V223S mutants was described previously (23). The mutants were generated from the parent plasmid pLGPS (Fig. 1A), which expresses the MLV gag and pol genes from a truncated MLV long terminal repeat ( $\triangle \mathrm{LTR})$ promoter (41).

The targets of infection in this study included the murine fibroblast cell line NIH 3T3 and the human osteosarcoma cell line 143B (both obtained from the American Type Culture Collection). The ANGIE P cell line (Fig. 1B) is a D17-based (dog osteosarcoma) cell line expressing the construct pSV-A-MLVenv (obtained from the NIH AIDS Research and Reference Program) and pGA-1, an MLV-based retroviral vector (Fig. 1A) $(23,38)$. The expression construct pSV-A-MLVenv encodes the amphotropic MLV envelope gene, whereas pGA-1 expresses the bacterial $\beta$-galactosidase gene (lacZ) from the LTR promoter. In addition, pGA-1 also contains the neomycin phosphotransferase 
gene (neo), which is utilized as a selectable marker during infection. All cells were maintained in Dulbecco's modified Eagle's medium (ICN Biochemicals) supplemented with penicillin ( $50 \mathrm{U} / \mathrm{ml}$; Gibco), streptomycin ( $50 \mu \mathrm{g} / \mathrm{ml}$; Gibco), and bovine calf serum (6\% for ANGIE P and 143B cells or 10\% for NIH 3T3 cells; HyClone Laboratories).

Protocol for determining sensitivity of MLV RT to 3TC. The approach used to determine the sensitivity of MLV RT to 3TC is outlined in Fig. 1B. Briefly, either wildtype or mutated pLGPS along with $\mathrm{pSV} \alpha 3.6$, a plasmid that confers resistance to ouabain (30), were cotransfected into the ANGIE P cell line. Transfections were carried out by the previously described dimethyl sulfoxide-Polybrene method (28) and the transfected cells were then selected for resistance to $10^{-7} \mathrm{M}$ ouabain.

To determine the sensitivity of the MLV RTs to 3TC, we separately pooled and expanded more than 500 ouabain-resistant colonies for the wild-type and V223 mutants. For each pLGPS construct, $5 \times 10^{6}$ ouabain-resistant cells were plated on $100-\mathrm{mm}$ diameter dishes and the medium was changed $24 \mathrm{~h}$ later. Virus was harvested after another $24 \mathrm{~h}$ and serially diluted. In the presence of Polybrene $(50 \mu \mathrm{g} / \mathrm{ml})$, the virus was used to infect either NIH 3T3 or 143B cells for $4 \mathrm{~h}$. The target cells were plated at a density of $1-2 \times 10^{5}$ cells per $60-\mathrm{mm}$-diameter dish. In experiments conducted in the presence of the drug, the target cells were incubated with $10 \mu \mathrm{M} 3 \mathrm{TC} 4 \mathrm{~h}$ prior to infection, $4 \mathrm{~h}$ during infection, and $24 \mathrm{~h}$ post infection. The $3 \mathrm{TC}$ concentration used in this study was 15 - to 4000 -fold higher than the mean $50 \%$ inhibitory concentration $\left[\mathrm{IC}_{50}\right]$, ranging between $2.5 \mathrm{nM}$ to $0.67 \mu \mathrm{M}$, which was previously shown to inhibit several different strains of HIV-1 (9). The infected cells were then subjected to selection with G418, an analog of neomycin $(600 \mu \mathrm{g} / \mathrm{ml}$ for 143B and D17 cells and $1.2 \mathrm{mg} / \mathrm{ml}$ for NIH 
$3 \mathrm{~T} 3$ cells) $24 \mathrm{~h}$ after infection. The effect of 3TC treatment on MLV replication (wildtype and V223 mutants) was determined by the number of drug-resistant colonies obtained in the presence or absence of 3TC.

Comparison of viral titers in the presence or absence of 3TC. Viral titers were determined by quantitation of G418-resistant NIH 3T3 and 143B cells after infection (the data are summarized in Table 1). ANGIE P cells transfected with wild-type pLGPS or the V223A, V223I, V223M, or V223S mutant were previously shown to produce infectious viral particles, and target cells infected with these viruses were expected to confer resistance to G418 (23). Two to four independent infections of NIH 3T3 and 143B cells, in the absence or presence of 3TC, were performed with virus containing either the wild-type pLGPS or one of the four V223 mutants.

Infection of NIH $3 \mathrm{~T} 3$ cells with the wild-type virus harvested from a single pool produced titers that ranged from $2.7 \times 10^{4}$ to $1.3 \times 10^{5} \mathrm{CFU} / \mathrm{ml}$ in the absence of 3TC. Treatment of the target cells with 3TC had no significant effect on the titers of the wildtype virus (82\%, relative to the untreated control). Similarly, infection of NIH 3 T3 cells with the V223M mutant produced titers that ranged from $1.6 \times 10^{2}$ to $9.2 \times 10^{4} \mathrm{CFU} / \mathrm{ml}$ in the absence of $3 \mathrm{TC}$ after harvesting virus from two different virus-producing pools. Treatment of the target cells with 3TC did not substantially affect the titers of the V223M mutant virus (57\%, relative to the untreated control) when compared to the inhibition of the luciferase-expressing HIV-1-based vector pNLuc (Fig. 2C). Only a 2-fold change was observed in the titers in the absence or presence of $3 \mathrm{TC}$, which is probably not significant considering the inherent variation that occurs during infections (23). We also assessed the sensitivity to 3TC of other V223 mutants (V223A, V223I, and V223S) in 
NIH 3T3 cells; the results were similar to those obtained with the wild-type and V223M mutant viruses. In summary, viral titers in the presence or absence of 3TC varied by only 2-fold, suggesting that 3TC did not significantly inhibit either the wild-type or V223 mutant RTs during infection of NIH $3 \mathrm{~T} 3$ cells.

To determine whether the lack of inhibition to MLV replication with 3TC treatment was specific to NIH 3T3 cells, we also tested infection of 143B cells and D17 cells. Infection of 143B cells with virus containing either the wild-type or V223M mutant RT produced results similar to those obtained with NIH 3T3 cells. Virus obtained from wild-type pLGPS produced viral titers that ranged from $3.4 \times 10^{2}$ to $1.9 \times 10^{3}$ $\mathrm{CFU} / \mathrm{ml}$ in the absence of 3TC. Treatment of the target cells with 3TC had no significant effect on the titers of the wild-type virus (110\%, relative to the untreated control). In addition, infection of 143B cells with virus containing the V223M mutant RT produced viral titers ranging from $1.0 \times 10^{1}$ to $2.5 \times 10^{2} \mathrm{CFU} / \mathrm{ml}$ in the absence of $3 \mathrm{TC}$ and $0.5 \times$ $10^{1}$ to $1.3 \times 10^{2} \mathrm{CFU} / \mathrm{ml}$ in the presence of $3 \mathrm{TC}(57 \%$, relative to the untreated control). Similar results were obtained with D17 cells (data not shown).

Activation of 3TC in target cells. The results obtained with viruses generated and harvested from the ANGIE P cells that had been transfected with either the wild-type or V223 mutant constructs showed that all MLV RTs were resistant to 3TC (Table 1). There are two possible explanations for these results. First, structural differences between MLV RT and HIV-1 RT might account for the resistance of MLV RT to 3TC. Second, the uptake and/or phosphorylation of the drug by the target cells might be inefficient. To address these possibilities, we generated infectious HIV-1 particles and used them to infect the various target cells as previously described (32, 33) (Fig. 2A and 
2B). Briefly, 293T (human embryonic kidney) cells were cotransfected with pNLuc and pSV-A-MLVenv. Pseudotyped virus stocks were harvested and used to infect NIH 3T3, HeLa, 143B, and D17 cells in the presence or absence of $10 \mu \mathrm{M}$ 3TC. The target cells infected in the presence of drug were incubated with $3 \mathrm{TC}$ for $4 \mathrm{~h}$ prior to infection, $4 \mathrm{~h}$ during infection, and $24 \mathrm{~h}$ postinfection. Two days postinfection, the cells were lysed and the amount of luciferase activity present in the lysates was measured with a luminometer (Tropix) (Fig. 2B).

Infection of NIH 3T3, 143B, and HeLa cells with the pNLuc-derived virus was decreased 20-fold by 3TC treatment (Fig. 2C). The D17 cells exhibited a 4-fold decrease in pNLuc expression relative to no 3TC treatment. Thus, in the same cell lines in which MLV infectivity was not significantly affected, HIV-1 infectivity was substantially reduced. These results indicate that the lack of an effect of 3TC on MLV infectivity was not due to problems associated with the uptake, phosphorylation, or other mechanisms that interfered with the inhibitory activity of the nucleoside analog in these target cells. The less efficient inhibition of pNLuc expression in the D17 cells in comparison to the other target cells (Fig. 2C) could have been caused by either reduced uptake or phosphorylation of $3 \mathrm{TC}$ in D17 cells. Alternatively, this cell line might actively export the nucleoside analog, thus reducing its efficacy. Regardless of the mechanism, HIV-1 infection of different cell types in vivo might display divergent susceptibilities to 3TC or other RT inhibitors. Thus, the data obtained with the D17 cell line might have implications for drug therapy in HIV-1-infected patients.

Mechanism of 3TC resistance in MLV RT. 3TC resistance arises in both retroviral (HIV-1, SIV, and FIV) and nonretroviral (HBV) polymerases with catalytic 
sites containing the YMDD motif $(1,8,17,31,48,49,55)$. This resistance usually results from a substitution of methionine to threonine, isoleucine or valine. Based on these observations, we expected that the wild-type MLV RT containing the YVDD motif would be resistant (44) and the V223M mutant would be sensitive to 3TC. It was therefore surprising that both the wild-type and the V223M mutant were highly resistant to $3 \mathrm{TC}$.

The mechanism by which the M184V mutant of HIV-1 RT confers resistance to 3TC is unclear. The methionine-to-isoleucine substitution at position 184 in HIV-1 RT results in a repositioning of the template-primer complex, and this rearrangement might result in a misalignment of the $3 \mathrm{TC}$ triphosphate with the template, resulting in a decrease in the turnover rate (47). Molecular modeling of the wild-type and M184I mutant of HIV-1 RT has also suggested that steric hindrance between the $\beta$-Loxathiolane ring of 3TC triphosphate and the $\beta$-branched amino acids (valine, isoleucine, and threonine) at position 184 interferes with $3 \mathrm{TC}$ binding (47). It is important to note that the proposed steric hinderance model does not preclude $3 \mathrm{TC}$ binding to RT in a mode that is unfavorable to its incorporation. In this regard, Feng and Anderson (13) reported that 3TC triphosphate binds to the M184V mutant with a much higher Kd value $(5.2 \mu \mathrm{M})$ relative to the wild type HIV-1 RT $(0.24 \mu \mathrm{M})$. Similarly, Wilson et al (56) suggested that $\beta$-L-2', $3^{\prime}$-dideoxy-5-fluoro- $3^{\prime}$-thiacytidine, a nucleoside analog that is structurally similar to $3 \mathrm{TC}$, binds with a higher affinity to the wild type HIV-1 RT than the M184V mutant. However, Krebs and coworkers did not find a substantial difference in the $\mathrm{Kd}$ values for $3 \mathrm{TC}$ binding to wild type and M184V mutants (35). Recent evidence indicates that the 3TC can bind to the M184V and M184I mutants of HIV-1 RT, 
which results in a conformational change in the enzyme that affects the nature of RNase H cleavages (16).

The fact that both the YMDD and YVDD motifs are highly resistant to 3TC strongly suggests that other structural determinants of MLV RT may interfere with the nature of 3TC binding through steric hindrance. The previous observation that MLV RT is sensitive to ddC (52) suggests that the steric hindrance involves the $\beta$-L-oxathiolane ring of 3TC. In accordance with the relatively low ( $25 \%)$ amino acid sequence identity between the MLV and HIV-1 RTs, structural alterations at or near the active site not related to the YVDD motif of MLV RT may lead to steric hindrance and prevent 3TC binding (18). A comparison of distances between residues of the YXDD motif and dNTP binding pocket of HIV-1 and MLV RTs reveals substantial differences (18, 24). Distances in MLV RT, specifically between the residues of the YVDD motif and K103, which is equivalent to K65 of HIV-1 RT (22), are more than 2 angstroms longer when compared to HIV-1 RT. It should be noted that the K65 residue of HIV-1 RT is associated with dual resistance to 3TC and ddC (19). These and other structural differences might contribute to the divergent sensitivities to 3TC.

Additionally, amino acid differences between the MLV and HIV-1 RTs around the dNTP-binding pocket may also provide the steric hindrance needed to confer 3TC resistance. Specifically, the MLV RT possesses a phenylalanine at position 155, which is equivalent to the tyrosine 115 in HIV-1 RT. The Y115 of HIV-1 RT has been shown to interact with the ribose ring of a dNTP substrate (25). Therefore, substitutions of the phenylalanine for the tyrosine in MLV RT dNTP binding site may alter the nature of 3TC binding. 
It is also conceivable that the structural differences near the active site or dNTP binding site between MLV and HIV-1 RTs, alter the affinity or nature of binding of 3TC triphosphate relative to dCTP by a mechanism not involving steric hinderance. The nature of the structural differences that confer high-level resistance to 3TC despite the presence of a YMDD motif appears to be unique to MLV RT, since several other retroviral RTs as well as HBV polymerase display sensitivity to 3TC when the YMDD motif is present $(1,8,49)$. Understanding the nature of the structural differences that lead to 3TC resistance in MLV RT might provide insights into the general mechanisms by which retroviral RTs acquire resistance to nucleotide analogs.

We especially thank Wei-Shau $\mathrm{Hu}$ for critical reading of the manuscript and valuable intellectual input and discussions throughout the project. We also especially thank Stephen H. Hughes for communicating unpublished results, intellectual input, and critical reading of the manuscript. We also thank Benjamin Beasley, Sara Cheslock, Que Dang, Krista Delviks, Carey Hwang, Timur Kabdulov, Terence Rhodes, Yegor Voronin, and Wen Hui Zhang for the critical reading of this manuscript and discussion of results. Finally, we extend our thanks to Ann Arthur for her editorial expertise and revisions.

\section{ACKNOWLEDGEMENTS}

This work was supported in part by Public Health Service grant CA58875 from the National Institutes of Health, HIV Drug Resistance Program, National Cancer Institute, and Laboratory of Molecular Microbiology, National Institute of Allergy and Infectious Diseases, National Institutes of Health. 
TABLE 1. The effect of 3TC treatment on MLV replication

\begin{tabular}{|c|c|c|c|c|c|}
\hline $\begin{array}{l}\text { Target } \\
\text { cells }\end{array}$ & $\begin{array}{l}\mathrm{MLV}^{a} \\
\mathrm{RT}^{a}\end{array}$ & $\begin{array}{l}\text { Expt. } \\
\text { no. }\end{array}$ & $\begin{array}{l}\text {-3TC titer } \\
(\mathrm{CFU} / \mathrm{ml})^{b}\end{array}$ & $\begin{array}{l}\text { + 3TC titer } \\
(\mathrm{CFU} / \mathrm{ml})^{c}\end{array}$ & $\begin{array}{c}+3 \mathrm{TC} \\
\text { relative titer } \\
(\text { mean } \pm \text { S. E. })^{d}\end{array}$ \\
\hline \multirow[t]{19}{*}{ NIH 3T3 } & WT & 1 & $3.7 \times 10^{4}$ & $2.6 \times 10^{4}$ & 0.70 \\
\hline & & 2 & $2.7 \times 10^{4}$ & $1.5 \times 10^{4}$ & 0.56 \\
\hline & & 3 & $7.2 \times 10^{4}$ & $9.2 \times 10^{4}$ & 1.28 \\
\hline & & 4 & $1.3 \times 10^{5}$ & $9.5 \times 10^{4}$ & 0.73 \\
\hline & & Avg & & & $0.82 \pm 0.16$ \\
\hline & V223M & 1 & $2.9 \times 10^{2}$ & $1.4 \times 10^{2}$ & 0.48 \\
\hline & & 2 & $1.6 \times 10^{2}$ & $1.0 \times 10^{2}$ & 0.63 \\
\hline & & 3 & $9.2 \times 10^{4}$ & $6.9 \times 10^{4}$ & 0.75 \\
\hline & & 4 & $2.4 \times 10^{4}$ & $1.0 \times 10^{4}$ & 0.42 \\
\hline & & Avg & & & $0.57 \pm 0.07$ \\
\hline & V223I & 1 & $3.4 \times 10^{4}$ & $2.5 \times 10^{4}$ & 0.74 \\
\hline & & 2 & $2.6 \times 10^{4}$ & $3.3 \times 10^{4}$ & 1.27 \\
\hline & & Avg & & & $1.01 \pm 0.27$ \\
\hline & V223A & 1 & $1.3 \times 10^{2}$ & $1.9 \times 10^{2}$ & 1.46 \\
\hline & & 2 & $2.1 \times 10^{2}$ & $2.0 \times 10^{2}$ & 0.95 \\
\hline & & Avg & & & $1.21 \pm 0.26$ \\
\hline & V223S & 1 & $4.3 \times 10^{4}$ & $4.3 \times 10^{4}$ & 1.00 \\
\hline & & 2 & $8.3 \times 10^{3}$ & $1.2 \times 10^{4}$ & 1.45 \\
\hline & & Avg & & & $1.23 \pm 0.23$ \\
\hline \multirow[t]{10}{*}{ 143B } & WT & 1 & $1.9 \times 10^{3}$ & $1.4 \times 10^{3}$ & 0.74 \\
\hline & & 2 & $3.4 \times 10^{2}$ & $5.8 \times 10^{2}$ & 1.70 \\
\hline & & 3 & $4.3 \times 10^{2}$ & $3.5 \times 10^{2}$ & 0.8 \\
\hline & & 4 & $1.2 \times 10^{3}$ & $1.3 \times 10^{3}$ & 1.10 \\
\hline & & Avg & & & $1.10 \pm 0.23$ \\
\hline & V223M & 1 & $2.5 \times 10^{2}$ & $1.3 \times 10^{2}$ & 0.52 \\
\hline & & 2 & $2.0 \times 10^{2}$ & $8.3 \times 10^{1}$ & 0.42 \\
\hline & & 3 & $1.3 \times 10^{1}$ & $1.1 \times 10^{1}$ & 0.85 \\
\hline & & 4 & $1.0 \times 10^{1}$ & $0.5 \times 10^{1}$ & 0.50 \\
\hline & & Avg & & & $0.57 \pm 0.09$ \\
\hline
\end{tabular}

${ }^{a}$ The genotype of MLV virus generated from transfection of pLGPS (wild-type or V223 mutants of MLV RT) into the ANGIE P encapsidating cell line.

${ }^{b}$ The titer obtained from infections in the absence of 3TC. The virus titer for each experimental group was determined by serial dilutions. In experiments using NIH 3T3 
target cells, four independent infections were performed with virus containing the wildtype or V223M mutant RT, whereas two independent infections were performed for the V223A, V223I, and V223S RTs. In experiments using 143B target cells, four independent infections were performed with virus containing the wild-type or V223M mutant RT.

${ }^{c}$ The titer obtained from infections in the presence of 3TC. The virus titer for each experimental group was determined by serial dilutions. Four independent infections were performed in the presence of $3 \mathrm{TC}(10 \mu \mathrm{M})$ as described in footnote $b$ for virus containing either the wild-type or V223M RT. The 3TC treatment included incubation of the target cells $4 \mathrm{~h}$ prior to infection, $4 \mathrm{~h}$ during infection, and $24 \mathrm{~h}$ postinfection.

${ }^{d}$ The +3TC relative titer was calculated as follows: [titer obtained in the presence of $3 \mathrm{TC}] \div$ [titer obtained in the absence of $3 \mathrm{TC}]$. The mean \pm standard error (S.E.) was determined by the Sigma Plot 5.0 program. 
FIG. 1. Structures of MLV-based constructs and protocol used to determine sensitivity to 3TC. (A) Structures of the MLV-based vector pGA-1 and the gag-pol expression construct pLGPS. The pGA-1 vector contains both LTRs and all cis-acting elements of MLV and transcribes the E. coli lacZ and neo from the LTR promoter. The internal ribosomal entry site (IRES) (26) of encephalomyocarditis virus is used to express neo. The construct pLGPS expresses the MLV gag-pol gene from a truncated viral LTR. (B) Experimental protocol. ANGIE P, a D17-based cell line expressing pGA-1 and a vector carrying the amphotropic MLV env gene (pSV-A-MLVenv), was separately transfected with either the wild-type (V223) or mutated (V223A, V223I, V223M, and V223S) pLGPS constructs. Virus from pools of these transfected ANGIE P cells was harvested and used to infect target cells (NIH 3T3 or 143B cells) for $4 \mathrm{~h}$ in the presence or absence of 3TC $(10 \mu \mathrm{M})$. The target cells were also treated with $3 \mathrm{TC}(10 \mu \mathrm{M}) 4 \mathrm{~h}$ preinfection and $24 \mathrm{~h}$ postinfection in the drug treatment groups. (C) Measurement of virus titer. The percent change in the virus titers of the wild-type and V223M mutant was measured in the absence or presence of $3 \mathrm{TC}(10 \mu \mathrm{M})$. Two to four independent experiments were performed for NIH 3T3 and 143B cells. Virus titers observed in the absence of 3TC treatment were defined as $100 \%$. 
GA-1
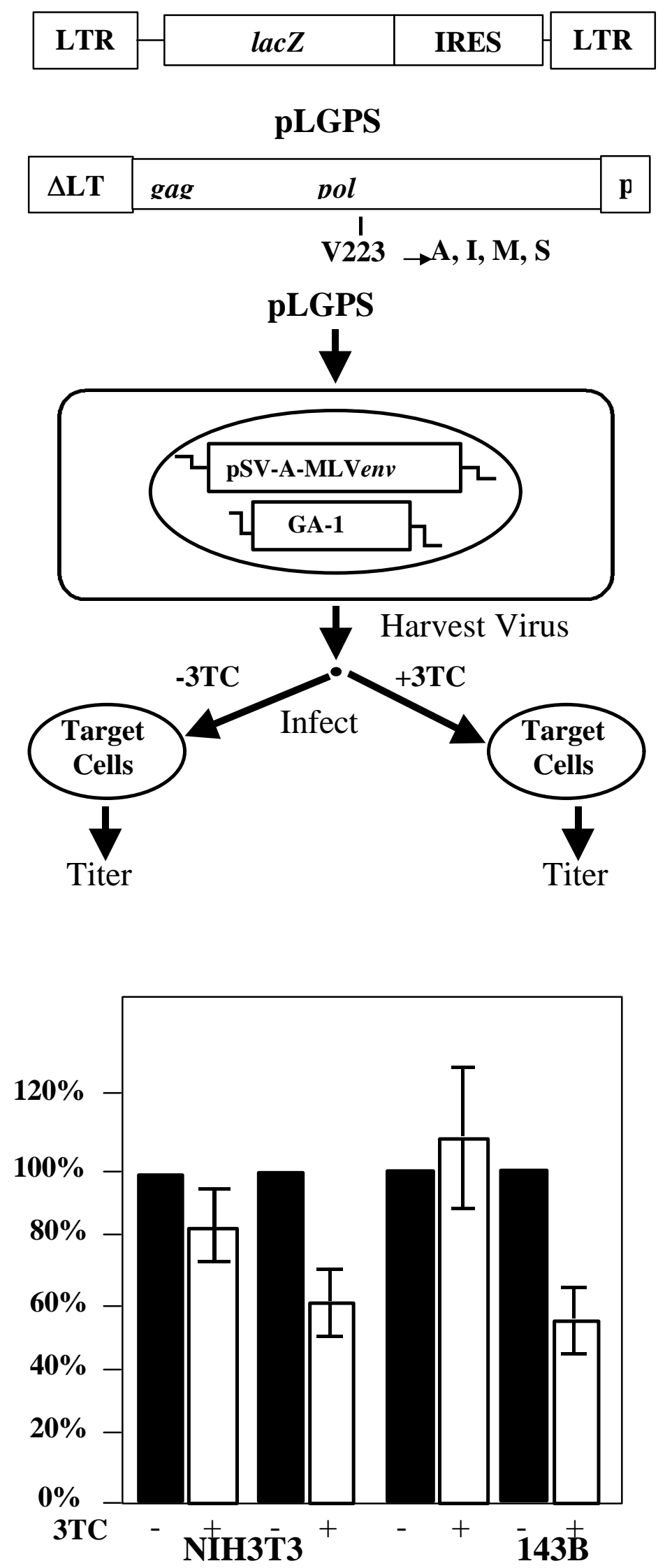
FIG. 2. Structure of HIV-1-based constructs and activation of $3 \mathrm{TC}$ in NIH 3T3, HeLA, 143B, and D17 cell lines. (A) Structure of the HIV-1-based luciferase-expression vector pNLuc, which contains both LTRs, other cis-acting elements required for viral replication, and the gag-pol gene of HIV-1. pNLuc expresses the luciferase reporter gene (luc) from the LTR promoter. (B) Experimental protocol. The vectors pNLuc and pSVA-MLVenv were cotransfected into $293 \mathrm{~T}$ cells and pseudotyped virus was used to infect various target cells (NIH 3T3, HeLA, 143B, and D17) for $4 \mathrm{~h}$ in the presence or absence of 3TC $(10 \mu \mathrm{M})$. The target cells were also treated with 3TC $(10 \mu \mathrm{M}) 4 \mathrm{~h}$ preinfection and $24 \mathrm{~h}$ postinfection in the drug treatment groups. (C) Measurement of chemiluminescence. The percent of chemiluminescence was measured in the absence or presence of 3TC $(10 \mu \mathrm{M})$. At least two independent experiments were performed for NIH 3T3, HeLA, 143B, and D17 cells. Chemiluminescence measurements in the absence of 3TC treatment were defined as $100 \%$. 
A.

\begin{tabular}{|c|c|c|c|}
\multicolumn{3}{c}{ pNLuc } \\
\hline LTR & gag pol & Luc & LTR \\
\hline
\end{tabular}

B.

Protocol for HIV-1

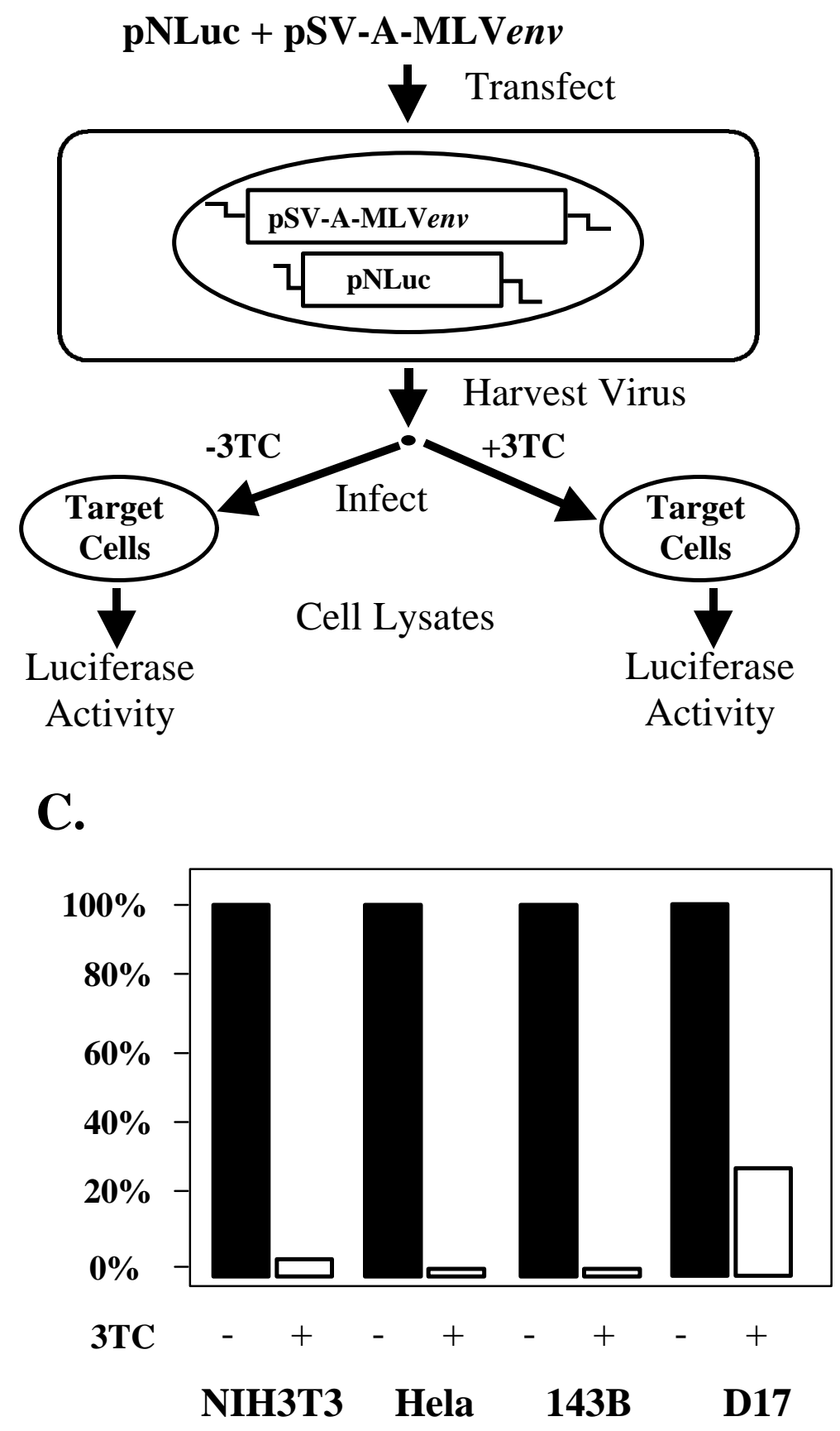




\section{REFERENCES}

1. Allen, M. I., M. Deslauriers, C. W. Andrews, G. A. Tipples, K. A. Walters, D. L. Tyrrell, N. Brown, and L. D. Condreay. 1998. Identification and characterization of mutations in hepatitis B virus resistant to lamivudine. Lamivudine Clinical Investigation Group. Hepatology 27:1670-1677.

2. Arts, E. J., and M. A. Wainberg. 1996. Mechanisms of nucleoside analog antiviral activity and resistance during human immunodeficiency virus reverse transcription. Antimicrob. Agents Chemother. 40:527-540.

3. Bakhanashvili, M., O. Avidan, and A. Hizi. 1996. Mutational studies of human immunodeficiency virus type 1 reverse transcriptase: the involvement of residues 183 and 184 in the fidelity of DNA synthesis. FEBS Lett. 391:257-262.

4. Balzarini, J., H. Pelemans, A. Karlsson, Q. E. De Clerc, and J. P. Kleim. 1996. Concomitant combination therapy for HIV infection preferable over sequential therapy with 3TC and non-nucleoside reverse transcriptase inhibitors. Proc. Natl. Acad. Sci. USA 93:13152-13157.

5. Boucher, C. A., N. Cammack, P. Schipper, R. Schuurman, P. Rouse, M. A. Wainberg, and J. M. Cameron. 1993. High-level resistance to (-) enantiomeric 2'-deoxy-3'-thiacytidine in vitro is due to one amino acid substitution in the catalytic site of human immunodeficiency virus type 1 reverse transcriptase. Antimicrob. Agents Chemother. 37:2231-2234.

6. Boyer, P. L., and S. H. Hughes. 1995. Analysis of mutations at position 184 in reverse transcriptase of human immunodeficiency virus type 1. Antimicrob. Agents Chemother. 39:1624-1628. 
7. Chang, C. N., S. L. Doong, J. H. Zhou, J. W. Beach, L. S. Jeong, C. K. Chu, C. H. Tsai, Y. C. Cheng, D. Liotta, and R. Schinazi. 1992. Deoxycytidine deaminase-resistant stereoisomer is the active form of (+/-)-2',3'-dideoxy-3'thiacytidine in the inhibition of hepatitis B virus replication [published erratum appears in J. Biol. Chem. 1992 Nov 25;267(33):24148]. J. Biol. Chem. 267:13938-13942.

8. Cherry, E., M. Slater, H. Salomon, E. Rud, and M. A. Wainberg. 1997. Mutations at codon 184 in simian immunodeficiency virus reverse transcriptase confer resistance to the (-) enantiomer of 2',3'-dideoxy- 3'-thiacytidine. Antimicrob. Agents Chemother. 41:2763-2765.

9. Coates, J. A., N. Cammack, H. J. Jenkinson, A. J. Jowett, M. I. Jowett, B. A. Pearson, C. R. Penn, P. L. Rouse, K. C. Viner, and J. M. Cameron. 1992. (-)2'-deoxy-3'-thiacytidine is a potent, highly selective inhibitor of human immunodeficiency virus type 1 and type 2 replication in vitro. Antimicrob. Agents Chemother. 36:733-739.

10. Coffin, J., M., S. H. Hughes, and H. E. Varmus. 1999. Retroviruses. Cold Spring Harbor Laboratory Press, Cold Spring Harbor, N.Y.

11. Cohen, J. 1997. The daunting challenge of keeping HIV suppressed. Science 277:32-33.

12. Cooley, T. P., L. M. Kunches, C. A. Saunders, J. K. Ritter, C. J. Perkins, C. McLaren, R. P. McCaffrey, and H. A. Liebman. 1990. Once-daily administration of 2',3'-dideoxyinosine (ddI) in patients with the acquired 
immunodeficiency syndrome or AIDS-related complex. Results of a Phase I trial. N. Engl. J. Med. 322:1340-1345.

13. Feng, J. Y., and K. S. Anderson. 1999. Mechanistic studies examining the efficiency and fidelity of DNA synthesis by the 3TC-resistant mutant (184V) of HIV-1 reverse transcriptase. Biochemistry 38:9440-9448.

14. Fischl, M. A., D. D. Richman, M. H. Grieco, M. S. Gottlieb, P. A. Volberding, O. L. Laskin, J. M. Leedom, J. E. Groopman, D. Mildvan, R. T. Schooley, G. G. Jackson, D. T. Durack, D. King and The AZT Collaborative Working Group. 1987. The efficacy of azidothymidine (AZT) in the treatment of patients with AIDS and AIDS-related complex. A double-blind, placebo-controlled trial. N. Engl. J. Med. 317:185-191.

15. Furman, P. A., M. Davis, D. C. Liotta, M. Paff, L. W. Frick, D. J. Nelson, R. E. Dornsife, J. A. Wurster, L. J. Wilson, J. A. Fyfe, J. V. Tuttle, W. H. Miller, L. Condreay, D. R. Averett, R. F. Schinazi, G. R. Painter. 1992. The antihepatitis B virus activities, cytotoxicities, and anabolic profiles of the (-) and (+) enantiomers of cis-5-fluoro-1-[2- (hydroxymethyl)-1,3-oxathiolan-5-yl]cytosine. Antimicrob. Agents Chemother. 36:2686-2692.

16. Gao, H.-Q., P. L. Boyer, S. G. Sarafianos, E. Arnold, and S. H. Hughes. Personal Communication.

17. Gao, Q., Z. Gu, M. A. Parniak, J. Cameron, N. Cammack, C. Boucher, and M. A. Wainberg. 1993. The same mutation that encodes low-level human immunodeficiency virus type 1 resistance to 2 ',3'-dideoxyinosine and 2',3'- 
dideoxycytidine confers high-level resistance to the (-) enantiomer of 2',3'dideoxy-3'- thiacytidine. Antimicrob. Agents Chemother. 37:1390-1392.

18. Georgiadis, M. M., S. M. Jessen, C. M. Ogata, A. Telesnitsky, S. P. Goff, and W. A. Hendrickson. 1995. Mechanistic implications from the structure of a catalytic fragment of Moloney murine leukemia virus reverse transcriptase. Structure 3:879-892.

19. Gu, Z., Q. Gao, H. Fang, H. Salomon, M. A. Parniak, E. Goldberg, J. Cameron, and M. A. Wainberg. 1994. Identification of a mutation at codon 65 in the IKKK motif of reverse transcriptase that encodes human immunodeficiency virus resistance to 2',3'-dideoxycytidine and 2',3'-dideoxy-3'-thiacytidine. Antimicrob. Agents Chemother. 38:275-281.

20. Gulick, R. M., J. W. Mellors, D. Havlir, J. J. Eron, C. Gonzalez, D. McMahon, L. Jonas, A. Meibohm, D. Holder, W. A. Schleif, J. H. Condra, E. A. Emini, R. Isaacs, J. A. Chodakewitz, and D. D. Richman. 1998. Simultaneous vs sequential initiation of therapy with indinavir, zidovudine, and lamivudine for HIV-1 infection: 100-week follow-up. JAMA 280:35-41.

21. Gulick, R. M., J. W. Mellors, D. Havlir, J. J. Eron, C. Gonzalez, D. McMahon, D. D. Richman, F. T. Valentine, L. Jonas, A. Meibohm, E. A. Emini, and J. A. Chodakewitz. 1997. Treatment with indinavir, zidovudine, and lamivudine in adults with human immunodeficiency virus infection and prior antiretroviral therapy. N. Engl. J. Med. 337:734-739.

22. Halvas, E. K., E. S. Svarovskaia, and V. K. Pathak. Unpublished Data. . 
23. Halvas, E. K., E. S. Svarovskaia, and V. K. Pathak. 2000. Development of an in vivo assay to identify structural determinants in murine leukemia virus reverse transcriptase important for fidelity. J. Virol. 74:312-319.

24. Hsiou, Y., J. Ding, K. Das, A. D. Clark, Jr., S. H. Hughes, and E. Arnold. 1996. Structure of unliganded HIV-1 reverse transcriptase at 2.7 A resolution: implications of conformational changes for polymerization and inhibition mechanisms. Structure 4:853-860.

25. Huang, H., R. Chopra, G. L. Verdine, and S. C. Harrison. 1998. Structure of a covalently trapped catalytic complex of HIV-1 reverse transcriptase: implications for drug resistance. Science 282:1669-1675.

26. Jang, S. K., H. G. Krausslich, M. J. Nicklin, G. M. Duke, A. C. Palmenberg, and E. Wimmer. 1988. A segment of the 5' nontranslated region of encephalomyocarditis virus RNA directs internal entry of ribosomes during in vitro translation. J. Virol. 62:2636-2643.

27. Kaushik, N., K. Singh, I. Alluru, and M. J. Modak. 1999. Tyrosine 222, a member of the YXDD motif of MuLV RT, is catalytically essential and is a major component of the fidelity center. Biochemistry 38:2617-2627.

28. Kawai, S., and M. Nishizawa. 1984. New procedure for DNA transfection with polycation and dimethyl sulfoxide. Mol. Cell. Biol. 4:1172-1174.

29. Kemp, S. D., C. Shi, S. Bloor, P. R. Harrigan, J. W. Mellors, and B. A. Larder. 1998. A novel polymorphism at codon 333 of human immunodeficiency virus type 1 reverse transcriptase can facilitate dual resistance to zidovudine and L-2',3'-dideoxy-3'-thiacytidine. J. Virol. 72:5093-5098. 
30. Kent, R. B., J. R. Emanuel, Y. Ben Neriah, R. Levenson, and D. E. Housman. 1987. Ouabain resistance conferred by expression of the cDNA for a murine $\mathrm{Na}+$, K+-ATPase alpha subunit. Science 237:901-903.

31. Keulen, W., N. K. Back, A. van Wijk, C. A. Boucher, and B. Berkhout. 1997. Initial appearance of the 184Ile variant in lamivudine-treated patients is caused by the mutational bias of human immunodeficiency virus type 1 reverse transcriptase. J. Virol. 71:3346-3350.

32. Kiernan, R. E., and E. O. Freed. 1998. Cleavage of the murine leukemia virus transmembrane env protein by human immunodeficiency virus type 1 protease: transdominant inhibition by matrix mutations. J Virol. 72:9621-9627.

33. Kiernan, R. E., A. Ono, G. Englund, and E. O. Freed. 1998. Role of matrix in an early postentry step in the human immunodeficiency virus type 1 life cycle. J. Virol. 72:4116-4126.

34. Kohlstaedt, L. A., J. Wang, J. M. Friedman, P. A. Rice, and T. A. Steitz. 1992. Crystal structure at 3.5 A resolution of HIV-1 reverse transcriptase complexed with an inhibitor. Science 256:1783-1790.

35. Krebs, R., U. Immendorfer, S. H. Thrall, B. M. Wohrl, and R. S. Goody. 1997. Single-step kinetics of HIV-1 reverse transcriptase mutants responsible for virus resistance to nucleoside inhibitors zidovudine and 3-TC. Biochemistry 36:10292-10300.

36. Kukhanova, M., S. H. Liu, D. Mozzherin, T. S. Lin, C. K. Chu, and Y. C. Cheng. 1995. L- and D-enantiomers of 2',3'-dideoxycytidine 5'-triphosphate 
analogs as substrates for human DNA polymerases. Implications for the mechanism of toxicity. J. Biol. Chem. 270:23055-23059.

37. Lafeuillade, A., C. Poggi, C. Tamalet, N. Profizi, C. Tourres, and O. Costes. 1997. Effects of a combination of zidovudine, didanosine, and lamivudine on primary human immunodeficiency virus type 1 infection. J. Infect. Dis. 175:10511055.

38. Landau, N. R., K. A. Page, and D. R. Littman. 1991. Pseudotyping with human T-cell leukemia virus type I broadens the human immunodeficiency virus host range. J. Virol. 65:162-169.

39. Larder, B. A., D. J. Purifoy, K. L. Powell, and G. Darby. 1987. Site-specific mutagenesis of AIDS virus reverse transcriptase. Nature 327:716-717.

40. Merigan, T. C., G. Skowron, S. A. Bozzette, D. Richman, R. Uttamchandani, M. Fischl, R. Schooley, M. Hirsch, W. Soo, C. Pettinelli, H. Schaumburg, and the ddC Study Group of the AIDS Clinical Trials Group. 1989. Circulating p24 antigen levels and responses to dideoxycytidine in human immunodeficiency virus (HIV) infections. A phase I and II study. Ann. Intern. Med. 110:189-194.

41. Miller, A. D., J. V. Garcia, N. von Suhr, C. M. Lynch, C. Wilson, and M. V. Eiden. 1991. Construction and properties of retrovirus packaging cells based on gibbon ape leukemia virus. J. Virol. 65:2220-2224.

42. Murray, H. W., K. E. Squires, W. Weiss, S. Sledz, H. S. Sacks, J. Hassett, A. Cross, R. E. Anderson, and L. M. Dunkle. 1995. Stavudine in patients with AIDS and AIDS-related complex: AIDS clinical trials group 089. J. Infect. Dis. 171 Suppl 2:S123-130. 
43. Poch, O., I. Sauvaget, M. Delarue, and N. Tordo. 1989. Identification of four conserved motifs among the RNA-dependent polymerase encoding elements. EMBO J. 8:3867-3874.

44. Powell, S. K., M. Artlip, M. Kaloss, S. Brazinski, R. Lyons, G. J. McGarrity, and E. Otto. 1999. Efficacy of antiretroviral agents against murine replicationcompetent retrovirus infection in human cells. J. Virol. 73:8813-8816.

45. Quan, Y., Z. Gu, X. Li, C. Liang, M. A. Parniak, and M. A. Wainberg. 1998. Endogenous reverse transcriptase assays reveal synergy between combinations of the $\mathrm{M} 184 \mathrm{~V}$ and other drug resistance-conferring mutations in interactions with nucleoside analog triphosphates. J. Mol. Biol. 277:237-247.

46. Rinaldo Jr, C. R., J. M. Liebmann, X. L. Huang, Z. Fan, Q. Al-Shboul, D. K. McMahon, R. D. Day, S. A. Riddler, and J. W. Mellors. 1999. Prolonged suppression of human immunodeficiency virus type 1 (HIV-1) viremia in persons with advanced disease results in enhancement of CD4 $\mathrm{T}$ cell reactivity to microbial antigens but not to HIV-1 antigens. J. Infect. Dis. 179:329-336.

47. Sarafianos, S. G., K. Das, A. D. Clark, Jr., J. Ding, P. L. Boyer, S. H. Hughes, and E. Arnold. 1999. Lamivudine (3TC) resistance in HIV-1 reverse transcriptase involves steric hindrance with beta-branched amino acids. Proc. Natl. Acad. Sci. USA 96:10027-10032.

48. Schinazi, R. F., R. M. Lloyd, Jr., M. H. Nguyen, D. L. Cannon, A. McMillan, N. Ilksoy, C. K. Chu, D. C. Liotta, H. Z. Bazmi, and J. W. Mellors. 1993. Characterization of human immunodeficiency viruses resistant to oxathiolanecytosine nucleosides. Antimicrob. Agents Chemother. 37:875-881. 
49. Smith, R. A., K. M. Remington, R. M. Lloyd, Jr., R. F. Schinazi, and T. W. North. 1997. A novel Met-to-Thr mutation in the YMDD motif of reverse transcriptase from feline immunodeficiency virus confers resistance to oxathiolane nucleosides. J. Virol. 71:2357-2362.

50. Smith, R. A., K. M. Remington, B. D. Preston, R. F. Schinazi, and T. W. North. 1998. A novel point mutation at position 156 of reverse transcriptase from feline immunodeficiency virus confers resistance to the combination of (-)-beta2',3'-dideoxy-3'-thiacytidine and 3'-azido-3'-deoxythymidine. J. Virol. 72:23352340.

51. Solder, B., U. Wintergerst, G. Notheis, J. Eberle, L. Gurtler, and B. H. Belohradsky. 1997. Effect of antiretroviral combination therapy (zidovudine/didanosine or zidovudine/lamivudine) on quantitative plasma human immunodeficiency virus-ribonucleic acid in children and adolescents infected with human immunodeficiency virus. J. Pediatr. 130:293-299.

52. Stair, R. K., C. J. Nelson, and J. W. Mellors. 1991. Use of recombinant retroviruses to characterize the activity of antiretroviral compounds. J. Virol. 65:6339-6342.

53. Tantillo, C., J. Ding, A. Jacobo-Molina, R. G. Nanni, P. L. Boyer, S. H. Hughes, R. Pauwels, K. Andries, P. A. Janssen, and E. Arnold. 1994. Locations of anti-AIDS drug binding sites and resistance mutations in the threedimensional structure of HIV-1 reverse transcriptase. Implications for mechanisms of drug inhibition and resistance. J. Mol. Biol. 243:369-387. 
54. Temesgen, Z., and A. J. Wright. 1999. Antiretrovirals. Mayo Clin Proc. 74:1284-301.

55. Tisdale, M., S. D. Kemp, N. R. Parry, and B. A. Larder. 1993. Rapid in vitro selection of human immunodeficiency virus type 1 resistant to 3'-thiacytidine inhibitors due to a mutation in the YMDD region of reverse transcriptase. Proc. Natl. Acad. Sci. USA 90:5653-5656.

56. Wilson, J. E., A. Aulabaugh, B. Caligan, S. McPherson, J. K. Wakefield, S. Jablonski, C. D. Morrow, J. E. Reardon, and P. A. Furman. 1996. Human immunodeficiency virus type- 1 reverse transcriptase. Contribution of Met-184 to binding of nucleoside 5'-triphosphate. J. Biol. Chem. 271:13656-13662. 


\section{CHAPTER 5}

Fidelity, Drug-Resistance, and the Evolution of Retroviruses 
Infections, latency and the steady state model. The progression of human immunodeficiency virus type 1 (HIV-1) infections involves three phases that includes the acute phase (primary infection), then clinical latency, and eventually, the clinical disease known as acquired immunodeficiency syndrome (AIDS) (18). The primary infection (18 weeks) is characterized by a burst of viral replication $\left(10^{6}\right.$ to $10^{9}$ particles/ $\mathrm{ml}$ of cellfree plasma) and decreases in $\mathrm{CD} 4^{+} \mathrm{T}$ cells counts that never completely rebound $(17,21$, 32). This is not only indicative of HIV-1 infections, because similar results are observed during the infection of macaque monkeys by simian immunodeficiency virus (SIV) (12).

The second phase, clinical latency (8-10 years), is not a time of viral inactivity and similar characteristics again are observed with both HIV-1 and SIV infections. This clinical latency is characterized by steady decreases in $\mathrm{CD} 4^{+} \mathrm{T}$ cells as well as for the continual and rapid replication of virus, which leads towards the progression of AIDS (2, $18,62,84)$. It has recently been proposed that a dynamic balance (steady state) exists between infection, cell death, cell replacement, and virus clearance which can be observed only when the system is perturbed, such as in the case with antiviral drugs (19, 44). It was calculated in this model that small changes in selective pressures have more of a drastic affect on the evolution of the virus than large increases in mutation rates. Therefore, it is believed that the high replication and mutation (to a lesser extent) rates of HIV-1 provide genetic variation, but selective forces drive the viral population's overall evolution. Nevertheless, it is important to investigate the mechanisms that govern fidelity, for without the initial variation that's generated, there would inevitably be no evolutionary potential present. Finally, the fidelity of the viral encoded polymerase 
identified as reverse transcriptase (RT) may be one of the major contributors of this generated variability observed in retroviral populations $(9,81)$.

Mutations and mutation rates of RTs. The in vivo mutation rates of several retroviruses have been measured to be $\sim 1.0 \times 10^{-5} / \mathrm{bp} /$ replication cycle and the spectrum of mutations occurring during a single cycle of replication were also determined (68-71, 78-80). The types of mutations included substitutions, frameshifts, deletions, deletions with insertions, duplications, and hypermutations. It has been proposed that these mutations could arise by three different mechanisms involving misincorporations, dislocation mutagenesis, or template switching events. (46, 47, 64, 79-81, 106). Substitutions are usually generated through the misincorporation of an incorrect nucleotide whereas frameshifts and $\mathrm{G}$ to $\mathrm{A}$ hypermutations occur by dislocation mutagenesis. Simple deletions, deletions with insertions, duplications, and complex hypermutations may arise by template switching events. In conclusion, reassortment of these various mutations can occur through both homologous and nonhomologous recombination $(47,64,106)$.

Mechanisms of fidelity. The mechanisms by which fidelity is manifested by polymerases are not completely known. It has been hypothesized that DNA polymerases may exploit a number of mechanisms to ensure accurate DNA synthesis. At a basic level, the initial discriminatory ability of polymerases may involve free energy differences between the correct and incorrect base pairs, increasing fidelity by 5 - to 150 fold (65). Furthermore, these differences in free energy could be magnified by the exclusion of water from the active site, providing additional accuracy during DNA synthesis (83). These two features alone may account for the high degree of precision 
exhibited by these enzymes in copying DNA. Beyond the initial screening of correct versus incorrect base pairs, the presence of a proofreading mechanism designated as the $3^{\prime}$ to $5^{\prime}$ exonuclease activity, may enhance fidelity through the removal of an incorporated mispaired nucleotide $(27,54)$. Finally, the highly processive nature observed in several DNA polymerase complexes involving interactions with additional accessory proteins, may also affect the ability of these enzymes to copy DNA correctly (57). Collectively, these factors may contribute to the highly accurate synthesis of DNA observed in nature and modifications in any one of these mechanisms may result in altered fidelity $(38,59)$.

Murine leukemia virus (MLV) RT active site. Comparison of several DNA polymerase crystal structures including MLV and HIV-1 RTs, have revealed a conserved tertiary organization between these various distinctly divergent DNA polymerases $(5,6$, $27,30,37,45,48,54,56,82,91,92)$. Their similar structures are indicative of a right hand with fingers, palm, and thumb, which may imply that all nucleotidyl transferases employ similar mechanisms during the polymerization of DNA, despite their divergent evolution. Our investigation, in accordance with previous observations, revealed the presence of a number of primary structures highly conserved between MLV and HIV-1 RTs $(37,45,85)$. These include the Thr-Val-Leu-Asp (TVLD), Asp-Ala-Tyr/Phe-Phe (DAXF), Leu-Pro-Gln-Gly (LPQG), and the Tyr-X-Asp-Asp (YXDD) amino acid motifs (Chapters 2 and 3). Analysis and comparison of crystal structures between these RTs, specifically in and around the active sites, demonstrate a similarity in the locations and orientations of several amino acid motifs (Fig. 1) (Chapter 3, Fig. 2) (37, 45). Further support is evidenced by a comparison of MLV and HIV-1 RT active sites with regard to 
distances measured between residues of the YXDD boxes and the dNTP-binding sites of the two enzymes (Tables 1 and 2). These lengths were determined by the RasMol program and were calculated as an average bond distance from a number of measurements taken between the various atoms of the side chain pairs inspected. The majority of the lengths were within $3 \AA$ (length of hydrogen bond) indicating that, indeed, the active sites of these two RTs (MLV and HIV-1) were alike. Similar results were obtained when analyzing the distances between residues constituting the dNTP-binding site of both MLV and HIV-1 RTs (Table 3 and 4). Collectively, this distance data as well as the mutational analysis of amino acids within the active site of MLV RT (Chapters 2 and 3) in accordance with crystal structure results, may allow for a more precise comparison and understanding of the two polymerases $(37,45,48,49,56)$. These results in turn may provide insight into the common mechanisms employed by all polymerases such as those governing catalysis, processivity, and fidelity.

Although biochemical and structural data characterizing these MLV RT amino acid motifs have been previously reported by a number of studies, our biochemical data presents additional evidence, of the importance of these residues for proper enzyme function. (Chapters 2 and 3) $(4,16,34,37,53,100)$. This importance is revealed in alterations in fidelity as well as reductions in both viral titers and RT activities as demonstrated by the V223 (YVDD motif) and the RNase H domain mutants of MLV RT (Chapter 2, Table 1 and Fig. 3). Similar findings were detected in our extensive mutational analysis of residues constituting the dNTP-binding site of MLV RT (Chapter 3, Tables 1-4). Our results also provide additional evidence that the MLV RT residues that we mutated are also present in HIV-1 RT, suggesting that different RTs $(\sim 25 \%$ 
amino acid homology) more than likely utilize similar mechanisms during DNA synthesis. This is displayed by the comparable changes in fidelity and reductions in RT activities which are observed in both MLV and HIV-1 RTs (Chapter 2 and 3) (10, 28, 61, 66, 104). For example, the YVDD motif in HIV-1 RT (M184V mutant) has been associated with a 2-fold increase in overall fidelity when compared to wild type (YMDD) (28). As expected, the presence of the YMDD motif in MLV RT (Chapter 2, Table 1) exhibited a 1.8-fold decrease in fidelity relative to the wild-type RT containing the YVDD motif. In addition, several of our V223 and dNTP-binding site mutants displayed similar RT activities as observed with HIV-1 RT (Chapter 2, Fig. 3) (10, 61).

Viral replication and RT activities. Although extensive mutational analysis has been performed on HIV-1 RT and to a lesser degree on MLV RT, the majority of these studies have been carried out in vitro $(7,10,14,16,41,53,61,77,99,105)$. A comparison of viral titers and RT activities obtained by both the V223 and the dNTPbinding site mutants in our study can be utilized to uncover possible correlations between in vivo and in vitro results. Generally, the reductions in viral titers of our MLV RT mutants were greater than the decreases in RT activities, suggesting that additional steps during reverse transcription were affected (Chapter 2, Fig. 2 and Chapter 3, Table 4). This is not unusual since in vivo, RT is required to polymerize DNA as well as perform two obligatory jumps.

We demonstrated that the V223 position of the highly conserved YVDD motif is necessary for proper polymerase function of MLV RT, which may indirectly affect viral replication (Chapter 2). In the case of the V223A, V223M, and V223S mutants of MLV RT and to a lesser extent the V223I mutant, a distinct correlation between reduction in 
titers and RT activities was evident (Chapter 2, Fig. 2). For example, the V223A and V223S mutants of MLV RT exhibited RT activities of $\sim 5 \%$ and viral titers of $\sim 2 \%$ in comparison to the wild-type RT, indicating that the reductions in titer were attributed to decreases in RT activities (Chapter 2, Table 1 and Fig. 2). Similar results, but not as pronounced, were observed by the remaining V223 mutants (V223I and V223M). This is also in agreement with the comparable mutants (M184 position) of the YMDD box in HIV-1 RT $(10,105)$.

Results obtained from our RNase H mutants imply that this MLV RT domain may play a crucial role during reverse transcription in vivo, but its influence on this process is not attributed to alterations in the ability of MLV RT to synthesize DNA. In contrast to the V223 mutants of MLV RT, the RNase H domain mutants possessed RT activities comparable to the wild-type enzyme with significant reductions in viral titers ranging between 2.5- to 4.8-fold when compared to the wild-type RT (Chapter 2, Table 2 and Fig. 2). This was expected since the mutations were not part of the polymerase active site. This is in accordance with previously published data, which shows that mutations in the RNase H domain of MLV RT may affect processivity as well as viral replication (7, 8, $36,100)$. Therefore, it is likely that an alteration in processivity by these RNase $\mathrm{H}$ mutants may account for the reductions in titers. It is possible that these mutants may not be able to complete reverse transcription efficiently, which would result in a lower number of proviruses being generated.

Surprisingly, many of our dNTP-binding site mutants in general exhibited greater decreases in RT activities in comparison to the homologous mutants at the equivalent positions in HIV-1 RT $(39,41,61,66,72,89)$. It is probable that these conflicting results 
may be attributed to differences between the two RTs despite their similarities (catalysis, amino acid motifs, and overall tertiary structures). The dNTP-binding site mutants also failed to show a correlation between viral titers and RT activities as seen with the V223 mutants of MLV RT (Fig. 2) (Chapter 2, Fig. 2 and Chapter 3, Table 4). However, our data suggests that a certain degree of RT activity is necessary to complete reverse transcription in vivo in the context of our assay (Chapter 3, Table 4). The majority of mutants displaying RT activities less than and equal to $3 \%$ exhibited reductions in viral titers $>10,000$-fold (no detectable viral titers) whereas mutants with RT activities of 5\% or greater produced detectable virus. The exceptions were the F155I, F155V, and the F156L mutants of MLV RT. Interestingly, the phenotype of the F155V mutant (RT activity $5.5 \%$ and no detectable viral titer) may be partly explained by its ability to incorporate both dNTPs and ribonucleotides (rNTPs) (34). Incorporation of rNTPs may promote degradation of the preintegrated viral DNA or inactivate the provirus, thus accounting for the $>10,000$-fold reduction in viral titers (Chapter 3, Table 4). This may also be the case for the F155I mutant of MLV RT (34).

The ability of the F155V or F155I mutants to incorporate rNTPs could be assessed by harvesting virus containing the mutant RTs, permeablizing the virus with nonionic detergent, and measuring the incorporation of labeled UTP through the utilization of an exogenous RT assay (Chapters 2 and 3). The relative rates of rNTP incorporation by these mutants can be determined through their comparison to wild-type MLV RT. In addition, increasing amounts of dNTPs in the presence of UTP could also confirm that both of these mutant RTs readily utilize rNTPs. If both substrates can be incorporated into a nascent DNA chain, increasing amounts of a dNTP should compete 
with the labeled UTP, resulting in a decrease of radiolabeled product. Detection of the RNA or RNA/DNA hybrid generated by these mutants may also require inactivation of the RNase $\mathrm{H}$ domain, which could recognize and potentially degrade these products.

As stated above, a majority of the dNTP-binding site mutants of MLV RT exhibited severe reductions of viral titers (> 10,000-fold) in comparison to wild-type RT, which correlate to decreases in RT activities $(<3 \%)$. The decreases in RT activity could be attributed to inefficient initiation of minus- or plus-strand synthesis, inefficient elongation, or an inability of RT to perform the obligatory jumps. It would be of interest to determine where these blocks occur during reverse transcription. This can be accomplished by infecting target cells with mutant virus, isolating preintegrated viral DNA, amplifying this DNA by PCR, and then resolving of the products by gel electrophoresis. Utilization of specific primer sets complementary to different regions of the viral genome could allow us to dissect the nature of the block. Also, the various mutations generating detectable virus could be introduced in the context of a replication competent virus and the growth kinetics of each mutant can be measured and compared to wild-type MLV. In conclusion, data obtained by this set of experiments may provide additional information on the role that these particular amino acids of MLV RT play during reverse transcription and viral replication.

Fidelity of MLV RT. Mutational analysis of the V223 position, the RNase H domain, and the dNTP-binding site of MLV RT indicate that a number of residues may play a role in the overall fidelity of this particular polymerase (Chapters 2, 3, and 4). However, the absence of large changes in fidelity (> 3-fold) observed with the mutants in our studies suggests that although specific amino acids within MLV RT may significantly 
influence the accuracy of DNA synthesis, other factors may also play an important role in fidelity. One possibility is that specific residues or amino acid motifs may not be as important as the overall structure of the protein. Several studies have illustrated that polymerases will undergo a number of conformational changes upon binding the template-primer complex and then the incoming $\operatorname{dNTP}(5,6,27,51,58,98)$. These conformational changes in turn may align the residues participating in catalysis with that of the incoming dNTP, allowing for incorporation only when the correct base pair is formed (92). Consequently, several mutations in combination may be necessary to observe alterations in fidelity greater than 3 -fold.

Another possibility is that polymerases such as MLV RT have evolved in a manner that optimizes several different properties, which work in concert with one another to ensure proper enzyme function. Therefore, it is possible that some residues of the RT may play critical roles in several of these enzymatic properties. In support of this, a number of amino acids, specifically within the dNTP-binding site, have been implicated with multiple functions in both MLV and HIV-1 RTs $(16,39,52,72,73,89,90)$. Therefore, dNTP-binding site mutations that alter fidelity may also modify the catalytic and processive attributes of the enzyme, due to the proximity of these residues to the active site $(37,48)$. This may account for the absence of hypermutators (highly inaccurate polymerases) in our extensive mutational analysis of MLV RT. In order to observe large changes in fidelity, we would probably also need to compensate for gross changes in other properties of the polymerase, which may have detrimental effects on the virus in the context of the in vivo assay. This is quite likely why the majority of the dNTP-binding site mutants of MLV RT exhibited severe reductions in viral titers and RT 
activities (Chapter 3, Table 4). One therefore could speculate that RTs are at a threshold, optimizing RT activity, processivity, and fidelity (or the amount of errors that could be tolerated by the virus). This optimization of RT properties by evolution may also explain the absence of the hypomutator (highly accurate polymerase) phenotype in these present studies. If polymerases are optimizing several properties simultaneously, they must have evolved to operate at the upper limits of their abilities. It would therefore be difficult to select or generate a more accurate polymerase with a substantial increase in fidelity without affecting RT activity or processivity. Hence, considerable reduction in fidelity may inevitably have a drastic effect on polymerase activity.

Perhaps, this paradox could be circumvented through the use of replication competent viruses harboring these various mutations and by allowing for the multiple rounds of replication. This may permit isolation of revertants with compensatory mutations that retain their mutator phenotype, yet are characterized by increases in RT activity. After several rounds of replication, infected clones could be isolated and the nature of the compensatory mutations could be determined by PCR analysis and sequencing. The mutant could be further characterized through the utilization of the rapid in vivo assay (Chapter 2). However, it is quite possible that no compensatory mutations would be isolated and the mutant virus will revert to a virus with wild-type RT, a phenotype that nature has selected already to operate at the optimum in regard to all these polymerase properties.

Protein-substrate interactions and flexibility of the dNTP-binding site. It has been proposed that DNA polymerases can distinguish between correct and incorrect base pairing by exploiting differences in the hydrogen bonding, shape, and free energy 
changes associated with a specific base pair (correct versus incorrect) $(27,54,65,75)$. The ability to exploit these differences could be attributed to the flexibility of the polymerase active site and/or to the specific interactions between the protein and its substrates. Therefore, mutations introduced within the active site of the polymerase could change the flexibility of this pocket or the affinity of the polymerase for its substrates, which could lead to alterations in the accuracy of DNA synthesis as observed with HIV-1 RT (41). These changes in turn would exaggerate or diminish the differences (hydrogen bonding, shape, and free energy) between correct and incorrect base pairs leading to an enzyme, which is more or less accurate.

Flexibility of the active site may encompass the dNTP-binding site and the adjacent area of the protein that interacts with the template-primer complex (specifically the base pair at the $\mathrm{N}$ position). It has also been proposed that a correctly formed Watson-Crick base pair will lie in an identical position in the minor groove of the DNA with a distinctive shape and interact with several conserved residues of the polymerase $(27,54,75)$. These protein-substrate interactions at least in the T7 DNA polymerase (and possibly in the DNA polymerase of Bacillus stearothermophilus) involve hydrogen bonding between R429 and the N3 of the primer 3'-terminus base and between Q615 and the $\mathrm{O} 2$ of the complementary base $(27,54)$. These protein-substrate interactions are prevented in the event of a mispair and result in a possible misalignment of altered substrate with residues of the active site. Substitutions introduced in the site accommodating the base pair could modify the local geometry of the area allowing for the proper fit of a mismatch. Mutations could also reposition or realign critical residues with the substrate in a manner that promotes catalysis. In addition, mutations at residues 
thought to directly contact either the dNTP or template-primer complex could alter the affinity of the polymerase for its substrates. Individually or cooperatively, these modifications may influence the accuracy of DNA synthesis.

Our results provide evidence in support of both hypotheses. We have demonstrated that certain modifications introduced into the active site of MLV RT (YVDD motif and the dNTP-binding site) can have statistically significant effects on the fidelity of this particular polymerase (Chapter 2, Table 1 and Chapter 3, Tables 1, 2, and 3). Furthermore, depending on the amino acid position and the nature of the substitution (size) introduced into the active site of MLV RT, various degrees in the accuracy of the polymerase were encountered signifying that some residues may have a greater impact on fidelity than others.

Analysis of the MLV RT crystal structure reveals that the V223 side chain of the YVDD motif is in close proximity to the $3^{\prime} \mathrm{OH}$ group of the terminal nucleotide in the primer and thus, may position the template-primer complex during polymerization (37, 56). Our results support this hypothesis and we believe that the decreases in fidelity exhibited by the V223A, V223M, and the V223S MLV RT mutants probably result from the slippage of the template-primer complex with respect to the active site of the polymerase (Chapter 2, Table 1). This is likely, since the sequenced proviral DNAs from two infected clones displaying the white colony phenotype contained +1 frameshift mutations (Chapter 2, Fig. 3C). An increase in the frequency with which these slippage events occur could be influenced by decreases in the processive nature of the RT. This is in agreement with results obtained with mutants generated at the equivalent positions in HIV-1 RT (10). In accordance with previously published results, we propose that 
mutations in the dNTP-binding site could also affect protein-substrate interactions, particularly those between the polymerase and the incoming dNTP. For example, the F155W showed a decrease in fidelity by 2.8 fold when compared to the wild-type RT (Chapter 3, Table 1). This is in agreement with the homologous mutant (Y115W) of HIV-1 RT, which displayed increases in the frequency of misinsertions and mispair extensions attributed to a decrease in affinity for the correct dNTP and an increase in affinity for the incorrect $\mathrm{dNTP}(72,73)$. It is conceivable that mutations at residues D153 and A154 of MLV RT, which may directly contact the incoming dNTP, could also have an influence on protein-substrate interactions. However, it is noteworthy that the interactions between these residues and the dNTP substrate involve the main chain and therefore, substitutions in the side chains should still preserve the contacts (48).

We can speculate that a number of the MLV RT mutants may exhibit decreases in fidelity through an increase in the flexibility of the dNTP-binding site (Chapter 3, Tables 1, 2, and 3). This is evident for mutants of D153, A154, and F156 of MLV RT whose various side chain sizes were observed to have an effect on fidelity. For example, the presence of alanine (a small side chain) at position 153 reduced fidelity of the polymerase by 1.6-fold whereas a cysteine, glutamine, or serine had no effect in comparison to wildtype RT. Interestingly, it is probable that an arginine or phenylalanine (very large side chains) at this position drastically constrained the dNTP pocket of the RT to the point that all substrate dNTPs were excluded. Therefore, no viral titers were detectable for these mutants. Similar effects were observed with mutants of residues A154 and F156, which suggest that a side chain of $\sim 2$ carbon lengths or less (for A154) or > 3 carbon lengths (for F156) may be important in exerting effects on fidelity (Chapter 3, Tables 1 and 2). 
This may also be the case for several other mutants of MLV RT that are in close proximity to the dNTP-binding site, including T147A and K152A (Chapter 3, Table 3). The results imply that by reducing side chain size, the flexibility of the dNTP-binding site may increase, allowing for the accommodation of an incorrect base pair. This in turn could promote incorporation of the wrong nucleotide and result in inaccurate DNA synthesis. On a final note, the flexibility of the dNTP-binding site could also explain the hypermutator or hypomutator phenotypes if they should ever be observed. For example, a more open binding pocket for the substrate may give rise to a less stringent RT that indiscriminately incorporates mismatches such as with a hypermutator. In contrast, a decrease in this flexibility may constrain the binding pocket to the degree that only the correct base pair can be accommodated creating a more accurate RT.

Fidelity and drug-resistance. The similarities in MLV and HIV-1 RT structures as well as sensitivity profiles to dideoxynucleoside analogs makes MLV RT an attractive model system when it comes to dissecting the mechanisms by which drug-resistance arises in HIV-1 RT $(20,37,45,85,97,99)$. In contrast to these results, our study provides compelling evidence suggesting that at least in the case of the nucleoside analog 2', 3'-dideoxy-3'-thiacytidine (3TC), MLV RT may display the drug-resistant phenotype through a different mechanism than that viewed with HIV-1 RT (88). The YMDD motif of several RTs including HIV-1 RT has been associated with sensitivity to 3TC, whereas the YVDD, YIDD, and YTDD motifs have been shown to confer resistance to the drug $(15,35,93,94,102)$. In addition, the selection of the YVDD motif in the distinctly divergent polymerase of hepatitis B virus during 3TC treatment suggests that the development and the manifestation of resistance to this particular nucleoside analog may 
involve a universal mechanism $(1,13,20,31,99)$. Surprisingly, several MLV RT mutants containing the YMDD, YADD, as well as the YSDD motifs were all characterized with resistance to 3TC, in contrast to HIV-1 RT (Chapter 4, Table 1 and Fig. 2).

The administration of antiviral drugs in the course of an HIV-1 infection results in the selection of drug-resistant mutants $(19,99)$. From a different perspective, these drugresistant mutants that arise can be considered more accurate on the premise that they can distinguish between the normal substrate (dNTP) and the inhibitor, thus preventing the incorporation of the $\operatorname{drug}(39,60,102)$. Although, this inability to incorporate the nucleoside analog is sometimes up to 1000 times higher than wild type, the increase in fidelity is probably not mediated through the traditional manner since canonical WatsonCrick base pairing is preserved. For example, 3TC will base pair with a complementary $\mathrm{G}$ in the template. The mechanism by which this type of fidelity (resistance) is attained involves alterations in the local geometry of the dNTP-binding site and may be manifested through steric hindrance.

Recently, the crystal structure of the M184I mutant of HIV-1 RT containing the YIDD motif was resolved which revealed the nature by which resistance to 3TC arises (88). It was proposed that 3TC resistance entails steric hindrance between the oxathiolane ring of 3TC and the $\beta$-branched side chain of the isoleucine at position 184 . Our results imply that structural determinants other than the V223 position of MLV RT must account for this discrimination towards 3TC, which results in resistance. This natural propensity of MLV RT (wild type or V223 mutants) to exhibit resistance to 3TC may be attributed to differences in their dNTP-binding sites of MLV and HIV-1 RTs. 
For instance, amino acid(s) present in MLV RT and absent in HIV-1 RT could exert additional steric hindrance and therefore exclude the drug from the active site or reduce binding of the nucleoside analog, explaining the decreased efficacy of 3TC on MLV RT. It is also plausible that 3TC binding may involve additional residues besides the M184 position in HIV-1 RT which are absent in MLV RT and may consequently affect binding of the drug.

A sequence comparison of amino acids of the HIV-1, simian immunodeficiency virus (SIV), and feline immunodeficiency virus (FIV) RTs indicated the presence of a tyrosine at the conserved DAYF motif whereas MLV RT has a phenylalanine at the equivalent position (DAFF). Interestingly, the Y115 residue of HIV-1 RT has been proposed to be important for selection of dNTPs over rNTPs and has also been associated with drug resistance to Abacavir, a carbocyclic nucleoside analog $(48,101,103)$. A similar role has been proposed for the MLV RT equivalent (F155), at least in the case dNTP selectivity (37). Therefore, infection of target cells with the F155Y mutant of MLV RT (Chapter 3, Table 1) in the presence or absence of 3TC could reveal if indeed this position is the additional determinant conferring $3 \mathrm{TC}$ resistance.

Both the E89G and L74V mutants of HIV-1 RT have been shown to exhibit resistance to the $3 \mathrm{TC}$ and dideoxynucleoside analogs, respectively $(86,96)$. Comparing the amino acid sequences and the secondary structures of both MLV and HIV-1 RTs revealed that MLV RT lacks the E89 residue and has a valine at the equivalent L74 position of HIV-1 RT. Depending on sequence alignment, the homologous E89 residue in MLV RT may be an aspartic acid or a histidine. It is possible that any one of these residues may partake in an important role during the binding of the inhibitor. Since the 
glutamic acid and/or leucine side chains are absent in the equivalent positions in MLV RT, it is possible that both the wild-type and V223 mutant MLV RTs may have a natural propensity toward 3TC-resistance. Therefore, mutating these homologous residues in MLV RT with the YMDD box in the backbone may render the mutants sensitive to the nucleoside analog, 3TC. Alteration of the dNTP-binding site may change the flexibility of this pocket and ultimately have effects on the sensitivity profile of MLV RT for the drug. Further analysis may offer insights into how resistance is acquired and aid in the development of more potent antiviral drugs.

The role of structural and accessory proteins in fidelity. A hypothetical model of the HIV-1 replication complex during the initial stages of DNA synthesis illustrates the interaction of RT with a number of viral proteins including capsid (CA), matrix (MA), nucleocapsid (NC), integrase (IN), and the accessory protein, Vpr (67). There is compelling evidence showing that at least some of these proteins may be important for the overall fidelity of the RT complex. NC has previously been implicated with the efficiency of strand transfer in vitro whereas a Vpr deficient mutant of HIV-1 was observed to display a 4-fold increase in the in vivo mutation rate $(69,87)$. This model also proposes the possible involvement of IN, MA, and CA as well as several cellular proteins, which may contribute to fidelity of reverse transcription and whose roles are unclear at this time.

The structure and components of the MLV RT replication complex are currently unknown, but it may be similar to that of HIV-1. It would be interesting to investigate the role that other viral proteins, in conjunction with MLV RT, may play in regards to fidelity. Although MLV lacks accessory proteins like Vpr, several structural proteins of 
the virus including the MLV NC, CA, MA, or IN may exert an effect on fidelity. Extensive mutational analysis of any of these viral proteins could provide information on the characteristics of the in vivo RT complex and the effect that each particular protein has on accurate DNA synthesis. This can be accomplished by mutating either NC, CA, MA, or IN genes in pLGPS, transfecting these mutated plasmids into the ANGIE P cells, harvesting virus, and infecting target cells (Chapter 2). Any changes in the frequency of lac $Z$ inactivation in comparison to the wild type virus would signify that this particular viral protein was possibly part of the complex and important for fidelity. One of the disadvantages to this approach is that all of these proteins (NC, CA, MA, and IN) are necessary for other functions during the retroviral life cycle. Therefore, it is likely that the majority of the mutations may have deleterious effects on viral replication, integration, or assembly. This could be avoided by making conservative changes in these proteins.

Structural or accessory proteins may also affect the processivity of RT, which inevitably could influence fidelity. This may be characterized by increases or decreases in the occurrence of deletions, deletions with insertions, duplications, or complex hypermutations. In order to achieve highly processive DNA synthesis, many DNA polymerases may interact with additional proteins such as the multimeric protein ring designated as the sliding clamp (seen with E. coli DNA polymerase) or thioredoxin (seen with T7 DNA polymerase) $(27,57)$. These protein clamps and thioredoxin have been observed to encircle the template-primer complex and possibly prevent polymerase dissociation from the DNA. To date, no sliding clamp has been described for RT that would increase the overall processivity of the polymerase. However, growing evidence 
suggests that the protein aggregation (viral and cellular encoded proteins) of the in vivo RT complex could all hypothetically contribute to the processive nature of RT (67).

One aspect of the processive nature of RT that could feasibly be determined is the ability of RT to generate frameshift mutations. A cell line similar to the ANGIE P cells could be designed containing an inactivating mutation (-1 frameshift in a run of similar nucleotides) in the lacZ expressed from a retroviral vector within these cells. We could then screen a number of RT mutants that regenerate a functional reporter by determining the frequency of blue colonies to total number of colonies. A comparison of the mutants to the wild-type RT would give the relative change in the frequency with which lacZ is activated by a + frameshift.

In addition to cellular proteins that may alter fidelity directly through their interaction with RT, there are also a number of host proteins, other than RNA polymerase (see Chapter 1), that may affect the overall mutation rate of a given retrovirus. For instance, mutations introduced during plus-strand DNA synthesis of reverse transcription will result in a mismatch that can be corrected by host DNA repair machinery. The effects on fidelity by repair machinery have not been determined, but nevertheless they may significantly contribute to the overall accuracy with which DNA is synthesized. In addition, modification of viral RNA by the host cell's double-stranded RNA adenosine deaminase (dsRAD) can be another mechanism by which mutations arise $(11,40,55)$. It has been observed that dsRAD deaminates adenosines to inosines, which leads to A-to-G hypermutations. Therefore, the fidelity of reverse transcription may be affected by a number of proteins, both viral and cellular, in addition to RT. 
Evolution of retroviruses. Retroviruses are arranged in seven genera making up the Retroviridae family. Members of these genera exhibit considerable genetic variability, exemplified by the absence of any detectable homology between their nucleotide sequences (18). A substantial genetic divergence is also observed amongst the various groups within the Retroviridae genera encompassing the lentiviruses. However, amino acid sequence analysis of several viral genes has allowed for the classification (phylogenic trees) of all of these viruses and the data suggests that the majority of regions within these retroviral genomes have all co-evolved (24-26). In contrast, a different pattern is observed when analyzing amino acid sequences of env among various retroviruses. This data predicts that this part of the viral genome may have evolved through recombinational events shared between different viruses as observed with the generation of the mammalian type D viruses $(24,25,95)$. Therefore, taxonomic classification of these viruses (especially the lentiviruses) is important for providing insights into the evolution of these viruses, which are a potential concern to human health. In addition, the overall significance of RT's role in the generation of this genetic variation is unknown, yet it is of interest to speculate how much of this variability is attributed to the fidelity of this polymerase and how this in turn may affect the evolution of the viral population.

Origin and evolution of primate lentiviruses. It is hypothesized that primate lentiviruses evolved from a common ancestor 600-1200 years ago which suggests a phylogenetic relationship between human and monkey retroviruses possessing similar genetic organizations $(3,29,76)$. It has been hypothesized that members of the lentivirus genera radiated out from a common ancestor present in the Cercopithecus monkey 
species and the resultant viruses continued to evolve as a group in primates $(18,42,50)$. Analysis of genomic sequences has revealed five discrete groups of primate lentiviruses including groups of human immunodeficiency virus (HIV) types 1 and 2 as well as groups of simian immunodeficiency (SIV) (18). It has been shown that HIV-1 can be grouped with SIVcpz (SIV that infects chimpanzees) whereas HIV-2 possesses similarities to SIVsmm (SIV that infects the sooty mangabey monkeys). A number of these viruses, such as SIVsmm and SIVagm (SIV that infects African green monkeys), are both nonpathogenic in their respective hosts despite persistent life-long infections (18). Yet, cross-species transmission of SIVsmm into macaques (not the natural host) results in an acquired pathogenicity that leads to an AIDS-like disease $(22,63)$. Interestingly, HIV- 2 may have arisen by cross-species transmission of SIVsmm from sooty mangabey monkeys into humans $(23,33)$. The two viruses have similar sequences and inhabit overlapping regions in Africa $(43,74)$.

The story of HIV-1 is not as clear and can be explained by two hypotheses (23). One hypothesis suggests that the virus has always been present for some time but it was contained in isolated populations. Recently, the world has become a smaller place in light of urbanization as well as world travel and the virus can more easily spread. If this were the case, then you would expect that the virus would have had ample time to evolve with host and should be less pathogenic. An alternative hypothesis suggests that HIV-1 may have originated from another species such as chimpanzees due to its likeness to SIVcpz. This would explain HIV-1's pathogenicity and high mortality as well as increasing prevalence and it is quite possible that the virus is still evolving. 
In conclusion, due to the evolutionary potential of retroviruses, it is possible that HIV-1 will transform into a novel human pathogen wreaking more havoc than it already has caused. Recently, hantavirus and ebola virus outbreaks have shown how a virus can move quickly from one host to another with disastrous effects. The possibility in HIV-1 is almost endless due to its high replicative capacity and mutation rates, in addition to the millions of infected people. With all of this in mind, inaccurate DNA synthesis by the retroviral RT may therefore play a significant role in the generation of novel pathogens similar to HIV type 1 and 2, but with additional pathogenic properties.

Point mutations in cis-acting elements could increase expression (transcription or translation) of the retroviral genome, which would have an effect on the replicative capacity of the virus. For instance, augmentation in the replication of HIV-1 or 2 could potentially reduce the length of clinical latency (increase replication, increase viral load). This could involve changes in the viral promoter or internal ribosomal entry site. Changes in a number of viral proteins could also have dramatic effects in the pathogenicity of the virus. For example, mutations in the envelope glycoprotein of HIV1 and 2 may generate a virus with an altered cellular tropism so that this pathogen could infect a greater number of different cell types. In addition, a hypothetical recombinational event between HIV-1 and some other virus (influenza) could potentially generate HIV-1 that is spread as an aerosol and infects through the respiratory tract. Finally, HIV-1 or 2 could capture an oncogene creating a novel pathogen with the pathogenicity of HIV-1 and possessing a transforming power, which can give rise to a number of malignancies. This is observed with many different retroviruses (18). In light of this evolutionary potential exhibited by retroviruses, the effective management of both 
HIV-1 and HIV-2 infections as well as any novel variants (with additional pathogenic properties) arising from these viruses, will inevitably require the better understanding of RT fidelity and how drug-resistance develops. 
TABLE 1. Distance comparison of side chains between YXDD box and dNTP binding site residues of HIV-1 and MLV RTs.

\begin{tabular}{|c|c|c|c|c|c|c|c|c|}
\hline & $\mathrm{Y}^{183^{b}}$ & $\mathrm{Y}^{2222^{\mathrm{C}}}$ & M184 & V223 & D185 & D224 & D186 & D225 \\
\hline $\mathbf{K}^{\mathrm{a}}$ & $23.9^{\mathrm{d}}$ & 26.3 & 20.1 & 24.0 & 18.8 & 21.0 & 20.6 & 23.1 \\
\hline $\mathbf{R}$ & 20.7 & 20.3 & 17.7 & 17.8 & 12.8 & 15.4 & 18.2 & 18.0 \\
\hline D & 12.9 & 15.2 & 8.7 & 10.6 & 4.3 & 6.5 & 5.4 & 6.0 \\
\hline D & 19.2 & 19.6 & 15.5 & 13.4 & 8.6 & 9.7 & 14.7 & 13.8 \\
\hline $\mathbf{A}$ & 13.2 & 13.9 & 10.3 & 7.00 & 4.7 & 4.5 & 9.8 & 9.2 \\
\hline $\mathbf{Y} / \mathbf{F}$ & 9.0 & 11.1 & 8.8 & 6.1 & 8.00 & 6.5 & 11.6 & 10.7 \\
\hline Q & 14.2 & 15.0 & 12.3 & 10.6 & 8.9 & 11.2 & 14.1 & 13.2 \\
\hline
\end{tabular}

${ }^{a}$ Residues constituting the dNTP-binding site of MLV and HIV-1 RTs. For MLV RT, the residues include K103, R110, D153, A154, F155, and Q190 whereas the HIV-1 RT equivalents are K65, R72, D113, A114, Y115, and Q151, respectively.

${ }^{\mathrm{b}}$ Non-shaded columns represent the residues of HIV-1 RT YMDD motif, which include Y183, M184, D185, and D186 as observed in the HIV-1 RT structure [Hsiou, et al 1996].

${ }^{\mathrm{c}}$ Shaded columns represent the residues of MLV RT YVDD motif, which include Y222, V223, D224, D226 as observed in the MLV RT crystal structure [Georgiadis, et al 1995].

${ }^{\mathrm{d}}$ Distances are represented in Angstroms. Each distance is an average distance calculated from a number of measurements taken between two side chains, one constituting a residue of the dNTP-binding site and the other, of the YXDD motif of either MLV or HIV-1 RTs. Measurements were obtained from the RasMol program using either the HIV-1 RT crystal structure [Hsiou, et al 1996] or the MLV RT crystal structure [Georgiadis, et al 1995]. 
TABLE 2. Distance comparison of C $\alpha$-C $\alpha$ bonds between YXDD box and dNTP binding site residues of HIV-1 and MLV RTs.

\begin{tabular}{|c|c|c|c|c|c|c|c|c|}
\hline & $\mathrm{Y183}^{\mathrm{b}}$ & $\mathrm{Y}^{222^{\mathrm{c}}}$ & M184 & V223 & D185 & D224 & D186 & D225 \\
\hline $\mathbf{K}^{\mathrm{a}}$ & $26.0^{d}$ & 32.1 & 22.56 & 29.8 & 21.9 & 28.4 & 23.7 & 29.8 \\
\hline $\mathbf{R}$ & 23.7 & 26.3 & 19.98 & 23.5 & 18.7 & 22.1 & 21.7 & 24.2 \\
\hline D & 10.1 & 9.7 & 8.89 & 9.1 & 5.4 & 5.5 & 4.8 & 4.5 \\
\hline D & 14.2 & 14.4 & 11.00 & 11.3 & 8.3 & 8.8 & 11.7 & 11.7 \\
\hline A & 11.2 & 11.8 & 8.30 & 8.4 & 6.2 & 6.4 & 9.7 & 9.9 \\
\hline $\mathbf{Y} / \mathbf{F}$ & 12.5 & 13.1 & 9.36 & 9.4 & 8.7 & 8.8 & 12.5 & 11.2 \\
\hline $\mathbf{Q}$ & 15.8 & 16.3 & 12.09 & 12.9 & 11.9 & 15.5 & 16.1 & 18.6 \\
\hline
\end{tabular}

${ }^{a}$ Residues constituting the dNTP-binding site of MLV and HIV-1 RTs. For MLV RT, the residues include K103, R110, D153, A154, F155, and Q190 whereas the HIV-1 RT equivalents are K65, R72, D113, A114, Y115, and Q151, respectively.

a Non-shaded columns represent the residues of HIV-1 RT YMDD motif, which include Y183, M184, D185, D186 as observed in the HIV-1 RT structure [Hsiou, et al 1996].

${ }^{b}$ Shaded columns represent the residues of MLV RT YVDD motif, which include Y222, V223, D224, D225 as observed in the MLV RT crystal structure [Georgiadis, et al 1995].

${ }^{c}$ Distances are represented in Angstroms. Each distance is the measurement taken between two C $\alpha$ atoms, one from a residue constituting the dNTP-binding site and the other from a residue from the YXDD box of either MLV or HIV-1 RTs. Measurements were obtained from the RasMol program using either the HIV-1 RT crystal structure [Hsiou, et al 1996] or the MLV RT crystal structure [Georgiadis, et al 1995]. 
TABLE 3. Distance comparison of side chain distances between residues constituting the dNTP-binding site of HIV-1 or MLV RTs.

\begin{tabular}{|c|c|c|c|c|c|c|c|c|c|c|c|c|}
\hline & $\mathbf{K}^{\mathrm{a}}$ & $\mathbf{K}^{\mathrm{b}}$ & $\mathbf{R}$ & $\mathbf{R}$ & D & D & $\mathbf{A}$ & $\mathbf{A}$ & Y & $\mathbf{F}$ & $\mathbf{Q}$ & $\mathbf{Q}$ \\
\hline K & - & - & 14.0 & $8.3^{\mathrm{c}}$ & 19.4 & 18.7 & 22.2 & 23.7 & 21.5 & 22.5 & 17.2 & 17.2 \\
\hline $\mathbf{R}$ & - & - & - & - & 8.7 & 12.6 & 13.0 & 16.7 & 12.2 & 14.6 & 8.1 & 9.6 \\
\hline D & - & - & - & - & - & - & 6.2 & 6.7 & 9.3 & 8.4 & 9.0 & 11.6 \\
\hline $\mathbf{A}$ & - & - & - & - & - & - & - & - & 5.9 & 5.7 & 9.0 & 8.8 \\
\hline $\mathbf{Y} / \mathbf{F}$ & - & - & - & - & - & - & - & - & - & - & 6.0 & 5.4 \\
\hline $\mathbf{Q}$ & - & - & - & - & - & - & - & - & - & - & - & - \\
\hline
\end{tabular}

a Non-shaded columns represent the dNTP-binding site residues of HIV-1 RT, which include K65, R72, D113, A114, Y115, and Q151 as observed in the HIV-1 RT structure [Hsiou, et al 1996].

${ }^{\mathrm{b}}$ Shaded columns represent the dNTP-binding site residues of MLV RT, which include K103, R110, D153, A154, F155, and Q190 as observed in the MLV RT crystal structure [Georgiadis, et al 1995].

${ }^{c}$ Distances are represented in Angstroms. Each distance is an average distance calculated from a number of measurements taken between two side chains of residues constituting the dNTP-binding site of either MLV or HIV-1 RTs. Measurements were obtained from the RasMol program using either the HIV-1 RT crystal structure [Hsiou, et al 1996] or the MLV RT crystal structure [Georgiadis, et al 1995]. 
TABLE 4. Distance comparison of the C $\alpha-\mathrm{C} \alpha$ bonds between residues constituting the dNTP-binding sites of HIV-1 or MLV RTs.

\begin{tabular}{|c|c|c|c|c|c|c|c|c|c|c|c|c|}
\hline & $\mathbf{K}^{\mathrm{a}}$ & $\mathbf{K}^{\mathrm{b}}$ & $\mathbf{R}$ & $\mathbf{R}$ & D & D & $\mathbf{A}$ & $\mathbf{A}$ & $\mathbf{Y}$ & $\mathbf{F}$ & $\mathbf{Q}$ & $\mathbf{Q}$ \\
\hline $\mathbf{K}$ & - & - & $9.3^{\mathrm{c}}$ & 8.6 & 19.0 & 22.9 & 21.8 & 26.1 & 21.5 & 25.6 & 17.4 & 20.5 \\
\hline $\mathbf{R}$ & - & - & - & - & 13.3 & 15.5 & 16.1 & 18.6 & 15.4 & 17.7 & 11.6 & 12.9 \\
\hline D & - & - & - & - & - & - & 3.77 & 3.8 & 5.9 & 5.7 & 9.8 & 10.2 \\
\hline $\mathbf{A}$ & - & - & - & - & - & - & - & - & 3.9 & 3.8 & 9.7 & 9.5 \\
\hline $\mathbf{Y} / \mathbf{F}$ & - & - & - & - & - & - & - & - & - & - & 6.8 & 7.2 \\
\hline $\mathbf{Q}$ & - & - & - & - & - & - & - & - & - & - & - & - \\
\hline
\end{tabular}

${ }^{a}$ Non-shaded columns represent the dNTP-binding site residues of HIV-1 RT, which include K65, R72, D113, A114, Y115, and Q151 as observed in the HIV-1 RT structure [Hsiou, et al 1996].

${ }^{\mathrm{b}}$ Shaded columns represent the dNTP-binding site residues of MLV RT, which include K103, R110, D153, A154, F155, and Q190 as observed in the MLV RT crystal structure [Georgiadis, et al 1995].

${ }^{c}$ Distances are represented in Angstroms. Each distance is the measurement taken between the two $\mathrm{C} \alpha$ atoms of residues constituting the dNTP-binding site of either MLV or HIV-1 RTs. Measurements were obtained from the RasMol program using either the HIV-1 RT crystal structure [Hsiou, et al 1996] or the MLV RT crystal structure [Georgiadis, et al 1995]. 
FIG. 1. Comparison of the HIV-1 and MLV RT active sites. The MLV RT [Georgiadis, et al 1995] and HIV-1 RT [Hsiou, et al 1996] crystal structures were superimposed on one another using the RasMol program. HIV-1 RT active is represented as a ribbon where as MLV RT active site is illustrated as sticks. It each active site includes a portion of the fingers, the highly conserved DAY/F, LPQG, and YXDD motifs, as well as the primer grip. 


\section{Active Sites of MLV and HIV-1 RTs}

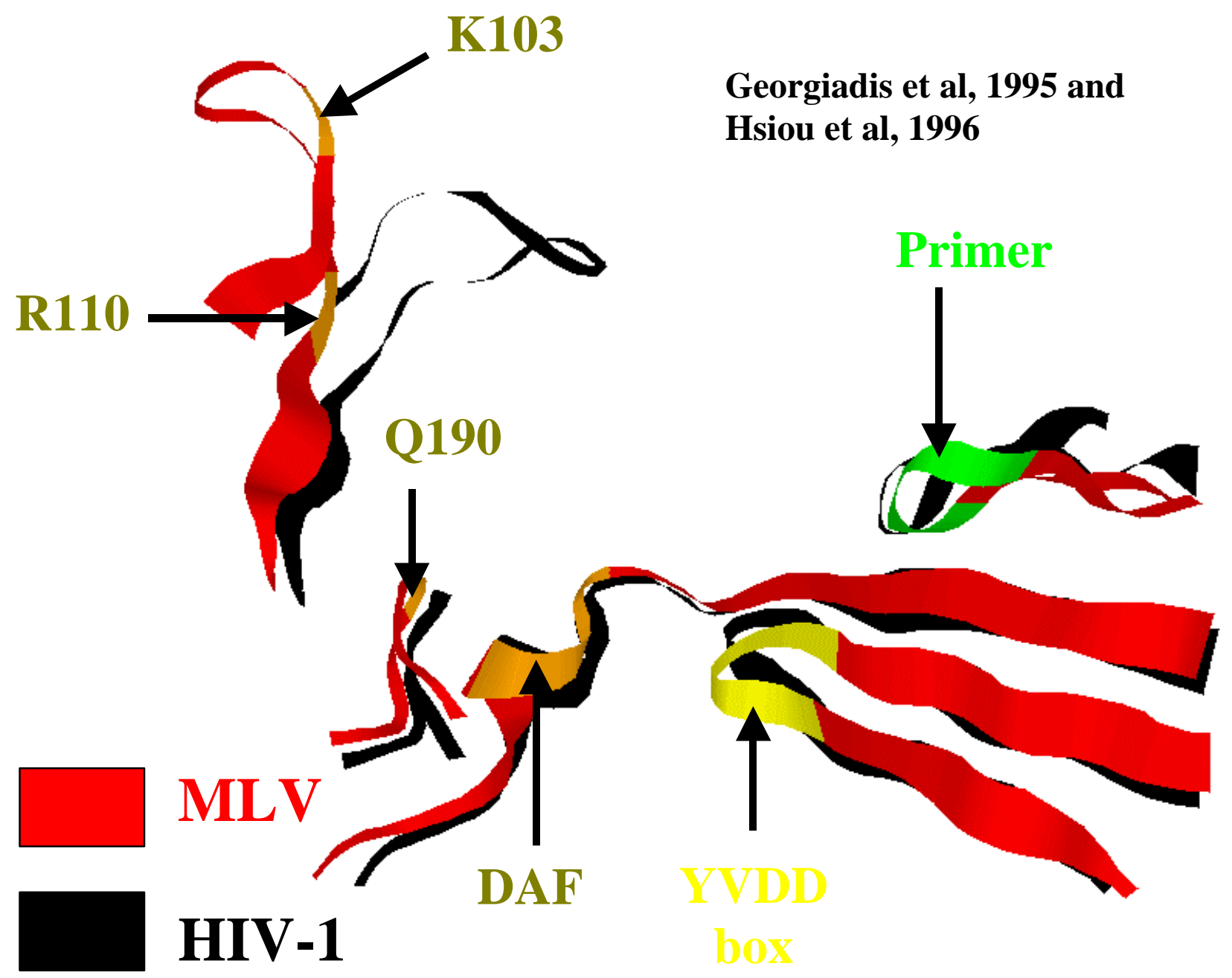


FIG. 2. Comparison of RT activities and viral titers of dNTP-binding site mutants. (A) Reverse transcriptase activity using virion-associated RT. The activities shown are relative to the RT activity determined for the wild type MLV RT (set to 100\%). The results represent an average of 2 to 3 experiments. The error bars represent the standard error of the mean and virus numbered as follows: 1; Wt, 2; K103R, 3; D153A, 4; D153C, 5; D153Q, 6; D153S, 7; A154S, 8; F155W, 9; F155Y, 10; Q190M. (B) Summary of virus titers obtained relative to the wild type viral titer (set to 100\%). The results represent an average of 2 to 13 experiments and the error bars represent the standard error of the mean. The $\%$ titer is in log scale. 

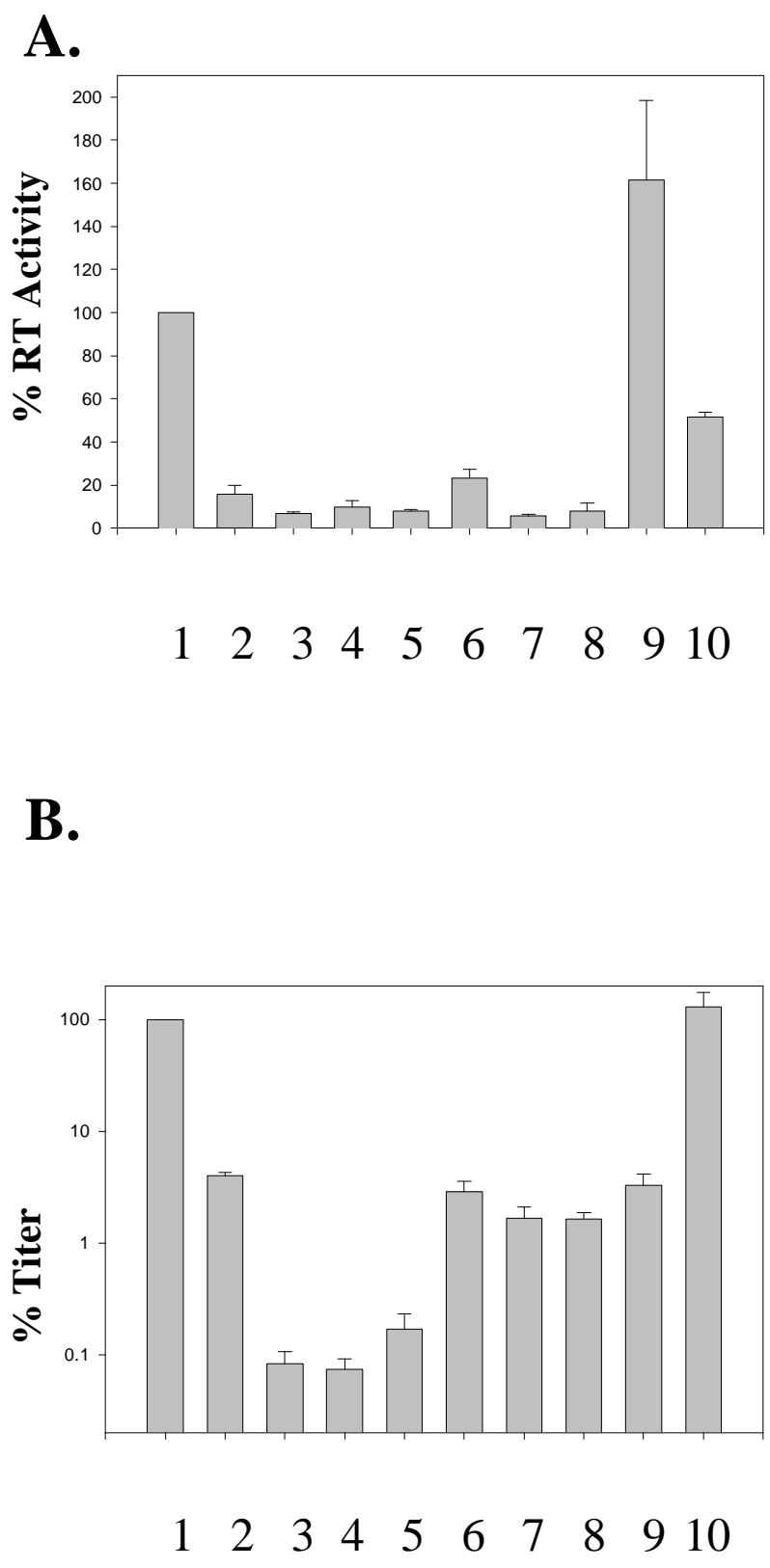


\section{REFERENCES}

1. Allen, M. I., M. Deslauriers, C. W. Andrews, G. A. Tipples, K. A. Walters, D. L. Tyrrell, N. Brown, and L. D. Condreay. 1998. Identification and characterization of mutations in hepatitis B virus resistant to lamivudine. Lamivudine Clinical Investigation Group. Hepatology 27:1670-1677.

2. Bacchetti, P., and A. R. Moss. 1989. Incubation period of AIDS in San Francisco. Nature 338:251-253.

3. Barre-Sinoussi, F. 1996. HIV as the cause of AIDS. Lancet 348:31-35.

4. Basu, A., V. B. Nanduri, G. F. Gerard, and M. J. Modak. 1988. Substrate binding domain of murine leukemia virus reverse transcriptase. Identification of lysine 103 and lysine 421 as binding site residues. J. Biol. Chem. 263:1648-1653.

5. Beese, L. S., V. Derbyshire, and T. A. Steitz. 1993. Structure of DNA polymerase I Klenow fragment bound to duplex DNA. Science 260:352-355.

6. Beese, L. S., J. M. Friedman, and T. A. Steitz. 1993. Crystal structures of the Klenow fragment of DNA polymerase I complexed with deoxynucleoside triphosphate and pyrophosphate. Biochemistry 32:14095-14101.

7. Blain, S. W., and S. P. Goff. 1995. Effects on DNA synthesis and translocation caused by mutations in the RNase $\mathrm{H}$ domain of Moloney murine leukemia virus reverse transcriptase. J. Virol. 69:4440-4452. 
8. Blain, S. W., and S. P. Goff. 1993. Nuclease activities of Moloney murine leukemia virus reverse transcriptase. Mutants with altered substrate specificities. J. Biol. Chem. 268:23585-23592.

9. Borman, A. M., C. Quillent, P. Charneau, K. M. Kean, and F. Clavel. 1995. A highly defective HIV-1 group O provirus: evidence for the role of local sequence determinants in G-->A hypermutation during negative- strand viral DNA synthesis. Virology 208:601-609.

10. Boyer, P. L., and S. H. Hughes. 1995. Analysis of mutations at position 184 in reverse transcriptase of human immunodeficiency virus type 1. Antimicrob. Agents Chemother. 39:1624-1628.

11. Cattaneo, R. 1994. Biased (A-->I) hypermutation of animal RNA virus genomes. Curr. Opin. Genet. Dev. 4:895-900.

12. Chakrabarti, L., M. Hurtrel, M. A. Maire, R. Vazeux, D. Dormont, L. Montagnier, and B. Hurtrel. 1991. Early viral replication in the brain of SIVinfected rhesus monkeys. Am. J. Pathol. 139:1273-1280.

13. Chang, C. N., V. Skalski, J. H. Zhou, and Y. C. Cheng. 1992. Biochemical pharmacology of (+)- and (-)-2',3'-dideoxy-3'-thiacytidine as anti-hepatitis B virus agents. J. Biol. Chem. 267:22414-22420.

14. Chao, S. F., V. L. Chan, P. Juranka, A. H. Kaplan, R. Swanstrom, and C. A. Hutchison, 3rd. 1995. Mutational sensitivity patterns define critical residues in the palm subdomain of the reverse transcriptase of human immunodeficiency virus type 1. Nucleic Acids Res. 23:803-810. 
15. Cherry, E., M. Slater, H. Salomon, E. Rud, and M. A. Wainberg. 1997. Mutations at codon 184 in simian immunodeficiency virus reverse transcriptase confer resistance to the (-) enantiomer of 2',3'-dideoxy- 3'-thiacytidine. Antimicrob. Agents Chemother. 41:2763-2765.

16. Chowdhury, K., N. Kaushik, V. N. Pandey, and M. J. Modak. 1996. Elucidation of the role of Arg 110 of murine leukemia virus reverse transcriptase in the catalytic mechanism: biochemical characterization of its mutant enzymes. Biochemistry 35:16610-16620.

17. Clark, S. J., M. S. Saag, W. D. Decker, S. Campbell-Hill, J. L. Roberson, P. J. Veldkamp, J. C. Kappes, B. H. Hahn, and G. M. Shaw. 1991. High titers of cytopathic virus in plasma of patients with symptomatic primary HIV-1 infection. N. Engl. J. Med. 324:954-960.

18. Coffin, J. M., S. H. Hughes, and H. E. Varmus. 1997. Retroviruses. Cold Spring Harbor Laboratory Press. Cold Spring Harbor, N.Y.

19. Coffin, J. M. 1995. HIV population dynamics in vivo: implications for genetic variation, pathogenesis, and therapy. Science 267:483-489.

20. Cohen, J. 1997. The daunting challenge of keeping HIV suppressed [news]. Science 277:32-33.

21. Daar, E. S., T. Moudgil, R. D. Meyer, and D. D. Ho. 1991. Transient high levels of viremia in patients with primary human immunodeficiency virus type 1 infection. N. Engl. J. Med. 324:961-964. 
22. Daniel, M. D., N. L. Letvin, N. W. King, M. Kannagi, P. K. Sehgal, R. D. Hunt, P. J. Kanki, M. Essex, and R. C. Desrosiers. 1985. Isolation of T-cell tropic HTLV-III-like retrovirus from macaques. Science 228:1201-1204.

23. Desrosiers, R. C. 1990. HIV-1 origins. A finger on the missing. Nature 345:288289.

24. Doolittle, R. F. 1989. Immunodeficiency viruses: the simian-human connection. Nature 339:338-339.

25. Doolittle, R. F., D. F. Feng, M. S. Johnson, and M. A. McClure. 1989. Origins and evolutionary relationships of retroviruses. Q. Rev. Biol. 64:1-30.

26. Doolittle, R. F., D. F. Feng, M. A. McClure, and M. S. Johnson. 1990. Retrovirus phylogeny and evolution. Curr. Top. Microbiol. Immunol. 157:1-18.

27. Doublie, S., S. Tabor, A. M. Long, C. C. Richardson, and T. Ellenberger. 1998. Crystal structure of a bacteriophage T7 DNA replication complex at $2.2 \mathrm{~A}$ resolution. Nature 391:251-258.

28. Drosopoulos, W. C., and V. R. Prasad. 1998. Increased misincorporation fidelity observed for nucleoside analog resistance mutations M184V and E89G in human immunodeficiency virus type 1 reverse transcriptase does not correlate with the overall error rate measured in vitro. J. Virol. 72:4224-4230.

29. Eigen, M., and K. Nieselt-Struwe. 1990. How old is the immunodeficiency virus? Aids 4:S85-93.

30. Eom, S. H., J. Wang, and T. A. Steitz. 1996. Structure of Taq polymerase with DNA at the polymerase active site. Nature 382:278-281. 
31. Furman, P. A., M. Davis, D. C. Liotta, M. Paff, L. W. Frick, D. J. Nelson, R. E. Dornsife, J. A. Wurster, L. J. Wilson, J. A. Fyfe, and et al. 1992. The antihepatitis B virus activities, cytotoxicities, and anabolic profiles of the (-) and (+) enantiomers of cis-5-fluoro-1-[2- (hydroxymethyl)-1,3-oxathiolan-5-yl]cytosine. Antimicrob. Agents Chemother. 36:2686-2692.

32. Gaines, H., M. A. von Sydow, L. V. von Stedingk, G. Biberfeld, B. Bottiger, L. O. Hansson, P. Lundbergh, A. B. Sonnerborg, J. Wasserman, and O. O. Strannegaard. 1990. Immunological changes in primary HIV-1 infection. Aids 4:995-999.

33. Gao, F., L. Yue, A. T. White, P. G. Pappas, J. Barchue, A. P. Hanson, B. M. Greene, P. M. Sharp, G. M. Shaw, and B. H. Hahn. 1992. Human infection by genetically diverse SIVSM-related HIV-2 in west Africa. Nature 358:495-499.

34. Gao, G., and S. P. Goff. 1998. Replication defect of moloney murine leukemia virus with a mutant reverse transcriptase that can incorporate ribonucleotides and deoxyribonucleotides. J. Virol. 72:5905-5911.

35. Gao, Q., Z. Gu, J. Hiscott, G. Dionne, and M. A. Wainberg. 1993. Generation of drug-resistant variants of human immunodeficiency virus type 1 by in vitro passage in increasing concentrations of 2',3'- dideoxycytidine and 2',3'-dideoxy3'-thiacytidine. Antimicrob. Agents Chemother. 37:130-133.

36. Garces, J., and R. Wittek. 1991. Reverse-transcriptase-associated RNaseH activity mediates template switching during reverse transcription in vitro. Proc. R. Soc. Lond. B. Biol. Sci. 243:235-239. 
37. Georgiadis, M. M., S. M. Jessen, C. M. Ogata, A. Telesnitsky, S. P. Goff, and W. A. Hendrickson. 1995. Mechanistic implications from the structure of a catalytic fragment of Moloney murine leukemia virus reverse transcriptase. Structure 3:879-892.

38. Glickman, B. W., V. A. Saddi, and J. Curry. 1994. International Commission for Protection Against Environmental Mutagens and Carcinogens. Working paper no. 2. Spontaneous mutations in mammalian cells. Mutat. Res. 304:19-32.

39. Gu, Z., Q. Gao, H. Fang, H. Salomon, M. A. Parniak, E. Goldberg, J. Cameron, and M. A. Wainberg. 1994. Identification of a mutation at codon 65 in the IKKK motif of reverse transcriptase that encodes human immunodeficiency virus resistance to 2',3'-dideoxycytidine and 2',3'-dideoxy-3'-thiacytidine. Antimicrob. Agents Chemother. 38:275-281.

40. Hajjar, A. M., and M. L. Linial. 1995. Modification of retroviral RNA by double-stranded RNA adenosine deaminase. J. Virol. 69:5878-5882.

41. Harris, D., N. Kaushik, P. K. Pandey, P. N. Yadav, and V. N. Pandey. 1998. Functional analysis of amino acid residues constituting the dNTP binding pocket of HIV-1 reverse transcriptase. J. Biol. Chem. 273:33624-33634.

42. Hayami, M., E. Ido, and T. Miura. 1994. Survey of simian immunodeficiency virus among nonhuman primate populations. Curr. Top. Microbiol. Immunol. 188: $1-20$.

43. Hirsch, V. M., R. A. Olmsted, M. Murphey-Corb, R. H. Purcell, and P. R. Johnson. 1989. An African primate lentivirus (SIVsm) closely related to HIV-2. Nature 339:389-392. 
44. Ho, D. D. 1997. Perspectives series: host/pathogen interactions. Dynamics of HIV-1 replication in vivo. J. Clin. Invest. 99:2565-2567.

45. Hsiou, Y., J. Ding, K. Das, A. D. Clark, Jr., S. H. Hughes, and E. Arnold. 1996. Structure of unliganded HIV-1 reverse transcriptase at 2.7 A resolution: implications of conformational changes for polymerization and inhibition mechanisms. Structure 4:853-860.

46. Hu, W. S., and H. M. Temin. 1990. Genetic consequences of packaging two RNA genomes in one retroviral particle: pseudodiploidy and high rate of genetic recombination. Proc. Natl. Acad. Sci. USA 87:1556-1560.

47. Hu, W. S., and H. M. Temin. 1990. Retroviral recombination and reverse transcription. Science 250:1227-1233.

48. Huang, H., R. Chopra, G. L. Verdine, and S. C. Harrison. 1998. Structure of a covalently trapped catalytic complex of HIV-1 reverse transcriptase: implications for drug resistance. Science 282:1669-1675.

49. Jacobo-Molina, A., J. Ding, R. G. Nanni, A. D. Clark, Jr., X. Lu, C. Tantillo, R. L. Williams, G. Kamer, A. L. Ferris, P. Clark, and et al. 1993. Crystal structure of human immunodeficiency virus type 1 reverse transcriptase complexed with double-stranded DNA at 3.0 A resolution shows bent DNA. Proc. Natl. Acad. Sci. USA 90:6320-6324.

50. Jin, M. J., H. Hui, D. L. Robertson, M. C. Muller, F. Barre-Sinoussi, V. M. Hirsch, J. S. Allan, G. M. Shaw, P. M. Sharp, and B. H. Hahn. 1994. Mosaic genome structure of simian immunodeficiency virus from west African green monkeys. EMBO J. 13:2935-2947. 
51. Kati, W. M., K. A. Johnson, L. F. Jerva, and K. S. Anderson. 1992. Mechanism and fidelity of HIV reverse transcriptase. J. Biol. Chem. 267:2598825997.

52. Kaushik, N., D. Harris, N. Rege, M. J. Modak, P. N. Yadav, and V. N. Pandey. 1997. Role of glutamine-151 of human immunodeficiency virus type-1 reverse transcriptase in RNA-directed DNA synthesis. Biochemistry 36:1443014438.

53. Kaushik, N., K. Singh, I. Alluru, and M. J. Modak. 1999. Tyrosine 222, a member of the YXDD motif of MuLV RT, is catalytically essential and is a major component of the fidelity center. Biochemistry 38:2617-2627.

54. Kiefer, J. R., C. Mao, J. C. Braman, and L. S. Beese. 1998. Visualizing DNA replication in a catalytically active Bacillus DNA polymerase crystal. Nature 391:304-307.

55. Kim, T., R. A. Mudry, Jr., C. A. Rexrode, 2nd, and V. K. Pathak. 1996. Retroviral mutation rates and A-to-G hypermutations during different stages of retroviral replication. J. Virol. 70:7594-7602.

56. Kohlstaedt, L. A., J. Wang, J. M. Friedman, P. A. Rice, and T. A. Steitz. 1992. Crystal structure at 3.5 A resolution of HIV-1 reverse transcriptase complexed with an inhibitor. Science 256:1783-1790.

57. Kong, X. P., R. Onrust, M. O'Donnell, and J. Kuriyan. 1992. Threedimensional structure of the beta subunit of E. coli DNA polymerase III holoenzyme: a sliding DNA clamp. Cell 69:425-437. 
58. Korolev, S., M. Nayal, W. M. Barnes, E. Di Cera, and G. Waksman. 1995. Crystal structure of the large fragment of Thermus aquaticus DNA polymerase I at 2.5-A resolution: structural basis for thermostability. Proc. Natl. Acad. Sci. USA. 92:9264-9268.

59. Kunkel, T. A., R. K. Hamatake, J. Motto-Fox, M. P. Fitzgerald, and A. Sugino. 1989. Fidelity of DNA polymerase I and the DNA polymerase I-DNA primase complex from Saccharomyces cerevisiae. Mol. Cell. Biol. 9:4447-4458.

60. Larder, B. A., and S. D. Kemp. 1989. Multiple mutations in HIV-1 reverse transcriptase confer high-level resistance to zidovudine (AZT). Science 246:11551158.

61. Larder, B. A., D. J. Purifoy, K. L. Powell, and G. Darby. 1987. Site-specific mutagenesis of AIDS virus reverse transcriptase. Nature 327:716-717.

62. Lemp, G. F., S. F. Payne, G. W. Rutherford, N. A. Hessol, W. Winkelstein, Jr., J. A. Wiley, A. R. Moss, R. E. Chaisson, R. T. Chen, D. W. Feigal, Jr., and et al. 1990. Projections of AIDS morbidity and mortality in San Francisco. JAMA 263:1497-1501.

63. Letvin, N. L., M. D. Daniel, P. K. Sehgal, R. C. Desrosiers, R. D. Hunt, L. M. Waldron, J. J. MacKey, D. K. Schmidt, L. V. Chalifoux, and N. W. King. 1985. Induction of AIDS-like disease in macaque monkeys with T-cell tropic retrovirus STLV-III. Science 230:71-73.

64. Linial, M., and D. Blair. 1984. Genetics of Retroviruses. In RNA tumor viruses (R. Weiss, N. Teich, H. Varmus, J. Coffin, Eds.) Vol. 1, pp.649-783. Cold Spring Harbor Press. Cold Spring Harbor, N.Y. 
65. Loeb, L. A., and T. A. Kunkel. 1982. Fidelity of DNA synthesis. Annu. Rev. Biochem. 51:429-457.

66. Lowe, D. M., V. Parmar, S. D. Kemp, and B. A. Larder. 1991. Mutational analysis of two conserved sequence motifs in HIV-1 reverse transcriptase. FEBS Lett. 282:231-234.

67. Mansky, L. M. 1997. Accessory replication proteins and the accuracy of reverse transcription: implications for retroviral genetic diversity. Trends Genet. 13:134136.

68. Mansky, L. M. 1996. Forward mutation rate of human immunodeficiency virus type 1 in a T lymphoid cell line. AIDS Res. Hum. Retroviruses 12:307-314.

69. Mansky, L. M. 1996. The mutation rate of human immunodeficiency virus type 1 is influenced by the vpr gene. Virology 222:391-400.

70. Mansky, L. M., and H. M. Temin. 1995. Lower in vivo mutation rate of human immunodeficiency virus type 1 than that predicted from the fidelity of purified reverse transcriptase. J. Virol. 69:5087-5094.

71. Mansky, L. M., and H. M. Temin. 1994. Lower mutation rate of bovine leukemia virus relative to that of spleen necrosis virus. J. Virol. 68:494-499.

72. Martin-Hernandez, A. M., E. Domingo, and L. Menendez-Arias. 1996. Human immunodeficiency virus type 1 reverse transcriptase: role of Tyr115 in deoxynucleotide binding and misinsertion fidelity of DNA synthesis. EMBO J. 15:4434-4442.

73. Martin-Hernandez, A. M., M. Gutierrez-Rivas, E. Domingo, and L. Menendez-Arias. 1997. Mispair extension fidelity of human immunodeficiency 
virus type 1 reverse transcriptases with amino acid substitutions affecting Tyr115. Nucleic Acids Res. 25:1383-1389.

74. Marx, P. A., Y. Li, N. W. Lerche, S. Sutjipto, A. Gettie, J. A. Yee, B. H. Brotman, A. M. Prince, A. Hanson, R. G. Webster, and et al. 1991. Isolation of a simian immunodeficiency virus related to human immunodeficiency virus type 2 from a west African pet sooty mangabey. J. Virol. 65:4480-4485.

75. Morales, J. C., and E. T. Kool. 1998. Efficient replication between nonhydrogen-bonded nucleoside shape analogs. Nat. Struct. Biol. 5:950-954.

76. Myers, G. 1994. Tenth anniversary perspectives on AIDS. HIV: between past and future. AIDS Res. Hum. Retroviruses 10:1317-1324.

77. Pandey, V. N., N. Kaushik, N. Rege, S. G. Sarafianos, P. N. Yadav, and M. J. Modak. 1996. Role of methionine 184 of human immunodeficiency virus type-1 reverse transcriptase in the polymerase function and fidelity of DNA synthesis. Biochemistry 35:2168-2179.

78. Parthasarathi, S., A. Varela-Echavarria, Y. Ron, B. D. Preston, and J. P. Dougherty. 1995. Genetic rearrangements occurring during a single cycle of murine leukemia virus vector replication: characterization and implications. J. Virol. 69:7991-8000.

79. Pathak, V. K., and H. M. Temin. 1990. Broad spectrum of in vivo forward mutations, hypermutations, and mutational hotspots in a retroviral shuttle vector after a single replication cycle: deletions and deletions with insertions. Proc. Natl. Acad. Sci. USA. 87:6024-6028. 
80. Pathak, V. K., and H. M. Temin. 1990. Broad spectrum of in vivo forward mutations, hypermutations, and mutational hotspots in a retroviral shuttle vector after a single replication cycle: substitutions, frameshifts, and hypermutations. Proc. Natl. Acad. Sci. USA 87:6019-6023.

81. Pathak, V. K. and W.-S. Hu. 1997. "Might as well jump" Template switching, defective genome formation, and recombination. Seminars in Virology 8:141-150.

82. Pelletier, H., M. R. Sawaya, A. Kumar, S. H. Wilson, and J. Kraut. 1994. Structures of ternary complexes of rat DNA polymerase beta, a DNA templateprimer, and ddCTP. Science 264:1891-1903.

83. Petruska, J., L. C. Sowers, and M. F. Goodman. 1986. Comparison of nucleotide interactions in water, proteins, and vacuum: model for DNA polymerase fidelity. Proc. Natl. Acad. Sci. USA 83:1559-1562.

84. Piatak, M., Jr., M. S. Saag, L. C. Yang, S. J. Clark, J. C. Kappes, K. C. Luk, B. H. Hahn, G. M. Shaw, and J. D. Lifson. 1993. Determination of plasma viral load in HIV-1 infection by quantitative competitive polymerase chain reaction. Aids 7 Suppl 2:S65-71.

85. Poch, O., I. Sauvaget, M. Delarue, and N. Tordo. 1989. Identification of four conserved motifs among the RNA-dependent polymerase encoding elements. EMBO J. 8:3867-3874.

86. Quan, Y., Z. Gu, X. Li, C. Liang, M. A. Parniak, and M. A. Wainberg. 1998. Endogenous reverse transcriptase assays reveal synergy between combinations of the M184V and other drug resistance-conferring mutations in interactions with nucleoside analog triphosphates. J. Mol. Biol. 277:237-247. 
87. Rodriguez-Rodriguez, L., Z. Tsuchihashi, G. M. Fuentes, R. A. Bambara, and P. J. Fay. 1995. Influence of human immunodeficiency virus nucleocapsid protein on synthesis and strand transfer by the reverse transcriptase in vitro. J. Biol. Chem. 270:15005-15011.

88. Sarafianos, S. G., K. Das, A. D. Clark, Jr., J. Ding, P. L. Boyer, S. H. Hughes, and E. Arnold. 1999. Lamivudine (3TC) resistance in HIV-1 reverse transcriptase involves steric hindrance with beta-branched amino acids. Proc. Natl. Acad. Sci. USA. 96:10027-10032.

89. Sarafianos, S. G., V. N. Pandey, N. Kaushik, and M. J. Modak. 1995. Glutamine 151 participates in the substrate dNTP binding function of HIV-1 reverse transcriptase. Biochemistry 34:7207-7216.

90. Sarafianos, S. G., V. N. Pandey, N. Kaushik, and M. J. Modak. 1995. Sitedirected mutagenesis of arginine 72 of HIV-1 reverse transcriptase. Catalytic role and inhibitor sensitivity. J. Biol. Chem. 270:19729-19735.

91. Sawaya, M. R., H. Pelletier, A. Kumar, S. H. Wilson, and J. Kraut. 1994. Crystal structure of rat DNA polymerase beta: evidence for a common polymerase mechanism. Science 264:1930-1935.

92. Sawaya, M. R., R. Prasad, S. H. Wilson, J. Kraut, and H. Pelletier. 1997. Crystal structures of human DNA polymerase beta complexed with gapped and nicked DNA: evidence for an induced fit mechanism. Biochemistry 36:1120511215.

93. Schinazi, R. F., R. M. Lloyd, Jr., M. H. Nguyen, D. L. Cannon, A. McMillan, N. Ilksoy, C. K. Chu, D. C. Liotta, H. Z. Bazmi, and J. W. Mellors. 1993. 
Characterization of human immunodeficiency viruses resistant to oxathiolanecytosine nucleosides. Antimicrob. Agents Chemother. 37:875-881.

94. Smith, R. A., K. M. Remington, R. M. Lloyd, Jr., R. F. Schinazi, and T. W. North. 1997. A novel Met-to-Thr mutation in the YMDD motif of reverse transcriptase from feline immunodeficiency virus confers resistance to oxathiolane nucleosides. J. Virol. 71:2357-2362.

95. Sonigo, P., L. Montagnier, P. Tiollais, and M. Girard. 1989. AIDS vaccines: concepts and first trials. Immunodefic. Rev. 1:349-366.

96. St Clair, M. H., J. L. Martin, G. Tudor-Williams, M. C. Bach, C. L. Vavro, D. M. King, P. Kellam, S. D. Kemp, and B. A. Larder. 1991. Resistance to ddI and sensitivity to AZT induced by a mutation in HIV-1 reverse transcriptase. Science 253:1557-1559.

97. Stair, R. K., C. J. Nelson, and J. W. Mellors. 1991. Use of recombinant retroviruses to characterize the activity of antiretroviral compounds. J. Virol. 65:6339-6342.

98. Steitz, T. A., S. Smerdon, J. Jager, J. Wang, L. A. Kohlstaedt, J. M. Friedman, L. S. Beese, and P. A. Rice. 1993. Two DNA polymerases: HIV reverse transcriptase and the Klenow fragment of Escherichia coli DNA polymerase I. Cold Spring Harb. Symp. Quant. Biol. 58:495-504.

99. Tantillo, C., J. Ding, A. Jacobo-Molina, R. G. Nanni, P. L. Boyer, S. H. Hughes, R. Pauwels, K. Andries, P. A. Janssen, and E. Arnold. 1994. Locations of anti-AIDS drug binding sites and resistance mutations in the three- 
dimensional structure of $\mathrm{HIV}-1$ reverse transcriptase. Implications for mechanisms of drug inhibition and resistance. J. Mol. Biol. 243:369-387.

100. Telesnitsky, A., and S. P. Goff. 1993. RNase H domain mutations affect the interaction between Moloney murine leukemia virus reverse transcriptase and its primer-template. Proc. Natl. Acad. Sci. USA 90:1276-1280.

101. Tisdale, M., T. Alnadaf, and D. Cousens. 1997. Combination of mutations in human immunodeficiency virus type 1 reverse transcriptase required for resistance to the carbocyclic nucleoside 1592U89. Antimicrob. Agents Chemother. 41:1094-1098.

102. Tisdale, M., S. D. Kemp, N. R. Parry, and B. A. Larder. 1993. Rapid in vitro selection of human immunodeficiency virus type 1 resistant to 3 -thiacytidine inhibitors due to a mutation in the YMDD region of reverse transcriptase. Proc. Natl. Acad. Sci. USA 90:5653-5656.

103. Vandamme, A. M., K. Van Vaerenbergh, and E. De Clercq. 1998. Anti-human immunodeficiency virus drug combination strategies. Antivir. Chem. Chemother. 9:187-203.

104. Wainberg, M. A., W. C. Drosopoulos, H. Salomon, M. Hsu, G. Borkow, M. Parniak, Z. Gu, Q. Song, J. Manne, S. Islam, G. Castriota, and V. R. Prasad. 1996. Enhanced fidelity of 3TC-selected mutant HIV-1 reverse transcriptase. Science 271:1282-1285.

105. Wakefield, J. K., S. A. Jablonski, and C. D. Morrow. 1992. In vitro enzymatic activity of human immunodeficiency virus type 1 reverse transcriptase mutants in 
the highly conserved YMDD amino acid motif correlates with the infectious potential of the proviral genome. J. Virol. 66:6806-6812.

106. Zhang, J., and H. M. Temin. 1993. Rate and mechanism of nonhomologous recombination during a single cycle of retroviral replication. Science 259:234238. 


\section{APPENDIX}

\section{Construction of MLV RT Mutants}


FIG. 1. Construction of the V223A, M, and S mutants of MLV RT. All three mutants were generated individually through the utilization of the plasmid pLGPS (template), either V223A, V223M, or V223S single-stranded DNA oligonucleotides (mutagenic primers), and a $5^{\prime}$ to $3^{\prime}$ mutagenesis kit. The plasmid pLGPS is an MLV gag-pol expression construct and Dam $^{-}$denotes that the plasmid DNA was non-methylated. In addition, YVDD represents the wild-type YXDD motif of MLV RT containing the V223 position coded by pol in pLGPS. To insure the absence of any undesired mutations introduced during mutagenesis, the BspEI-SalI inserts containing the appropriate mutations at position V223 (V223A, V223M, or V223S) were subcloned back into the backbone of pLGPS, which was also digested with both enzymes. Each individual mutant was screened by sequencing the BspEI-SalI fragments of each mutated pLGPS plasmids and the correct structure of these plasmids was confirmed by extensive restriction mapping. 


\section{A-1 Construction of V223A,M,S Mutants}

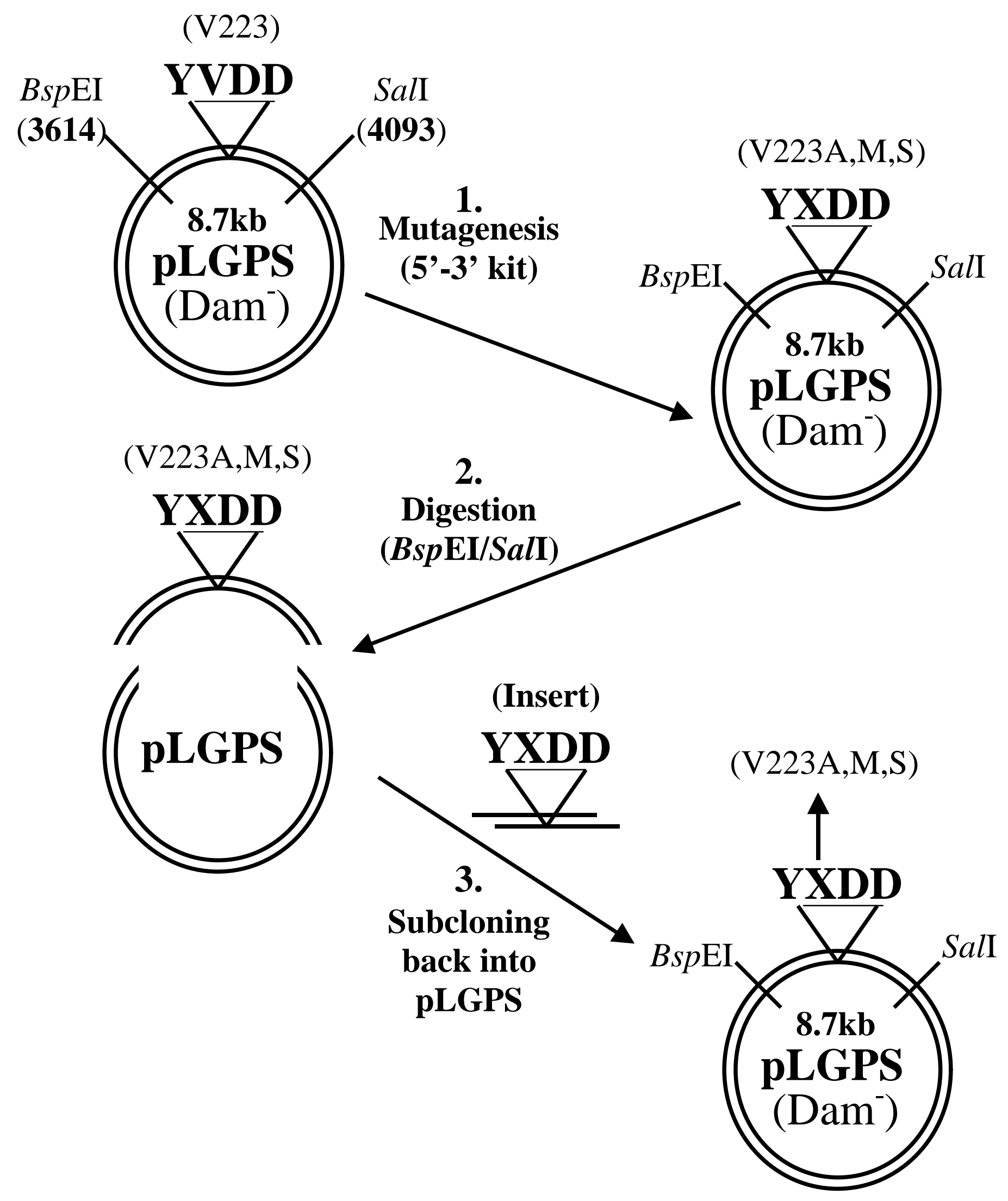


FIG. 2. Construction of the V223I mutant of MLV RT. The V223I mutant was generated through the utilization of the plasmid pLGPS (template), the V223I singlestranded DNA oligonucleotide (mutagenic primer), and a $5^{\prime}$ to $3^{\prime}$ mutagenesis kit. The plasmid pLGPS is an MLV gag-pol expression construct and Dam denotes that the plasmid DNA was non-methylated. In addition, YVDD represents the wild-type YXDD motif of MLV RT containing the V223 position coded by pol in pLGPS. To insure the absence of any undesired mutations introduced during mutagenesis, the BspEI-SalI inserts containing the appropriate mutation at position V223 (V223I) was subcloned back into the backbone of pLGPS, which was also digested with both enzymes. The V223I mutant was screened by digestion of plasmid with $C l a \mathrm{I}$, a new restriction site introduced during mutagenesis. The V223I mutation and the correct structure of the mutated plasmid were confirmed by the sequencing of the BspEI-SalI fragment and through extensive restriction mapping. 


\section{A-2 Construction of V223I Mutants}

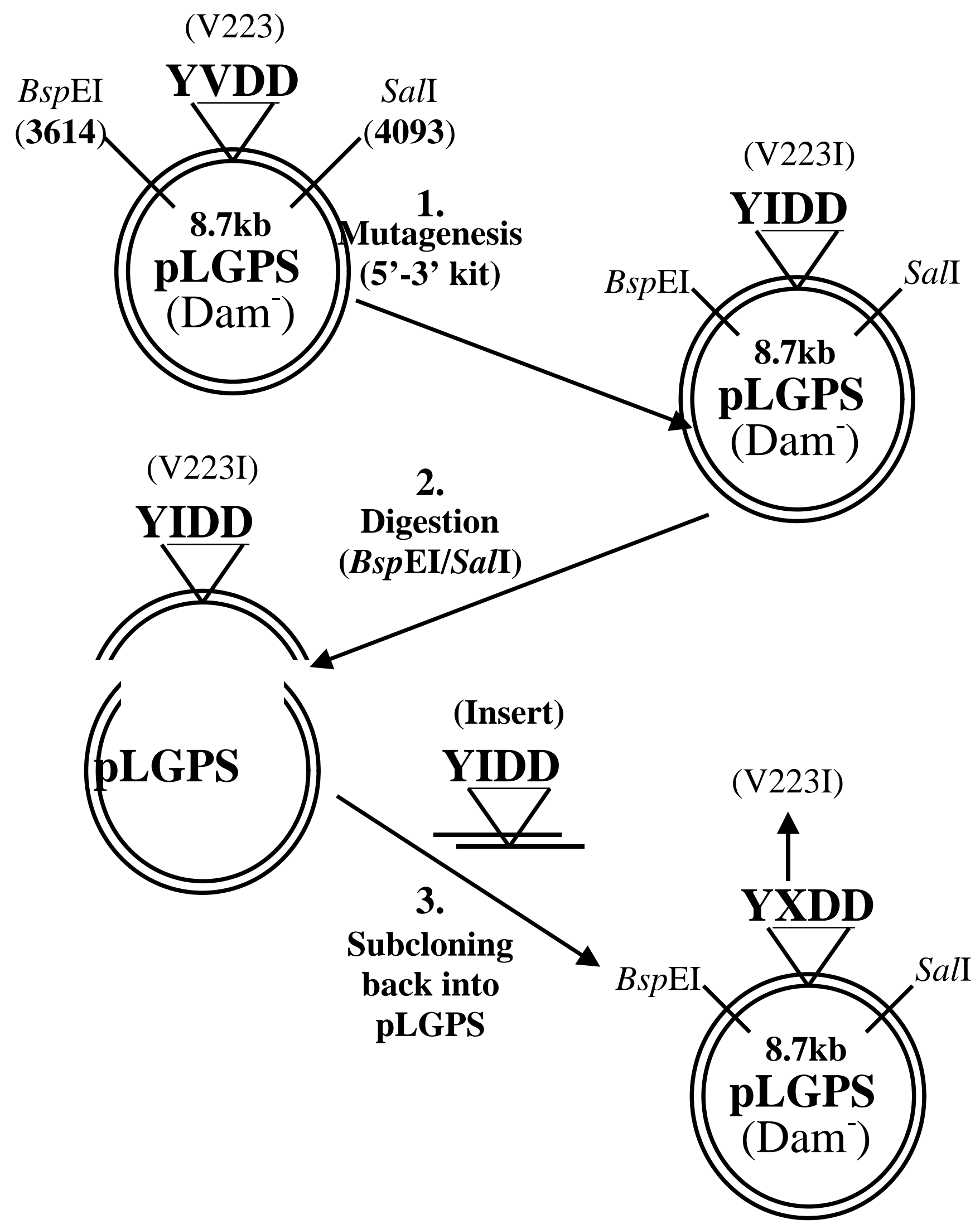


FIG. 3. Construction of the Q190E mutant of MLV RT. The Q190E mutant was generated through the utilization of the plasmid pLGPS (template), the Q190E singlestranded DNA oligonucleotide (mutagenic primer), and a Chameleon mutagenesis kit by Stratagene. The plasmid pLGPS is an MLV gag-pol expression construct and Dam ${ }^{-}$ denotes that the plasmid DNA was non-methylated. In addition, LPQG represents the wild-type LPQG motif of MLV RT containing the Q190 position coded by pol in pLGPS where as the LPEG contains the Q190E mutation. To insure the absence of any undesired mutations introduced during mutagenesis, the BclI-SalI insert containing the appropriate mutation at position Q190 (Q190E) was subcloned back into the backbone of pLGPS, which was also digested with both enzymes. The Q190E mutant was screened by digestion of plasmid with Bsu36I, a new restriction site introduced during mutagenesis. The Q190E mutation and the correct structure of the mutated plasmid were confirmed by the sequencing of the BclI-SalI fragment and through extensive restriction mapping. In addition, a pRMBNB derivative of Q190E (named pESQ190E) was also generated in the same manner described above with pRMBNB being utilized as the template instead of pLGPS. 


\section{A-3 Construction of Q190E Mutant}
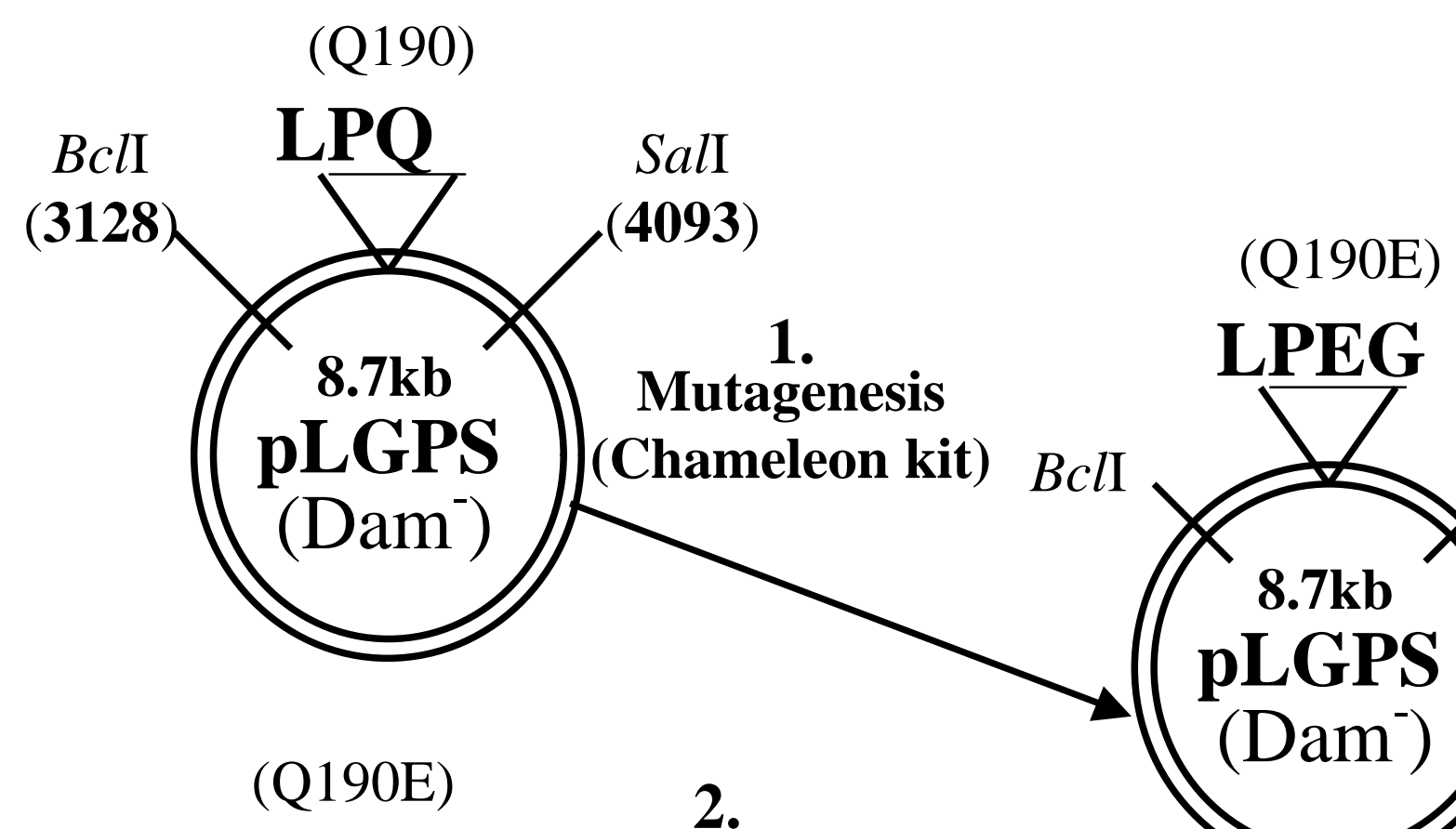
(Q190E)
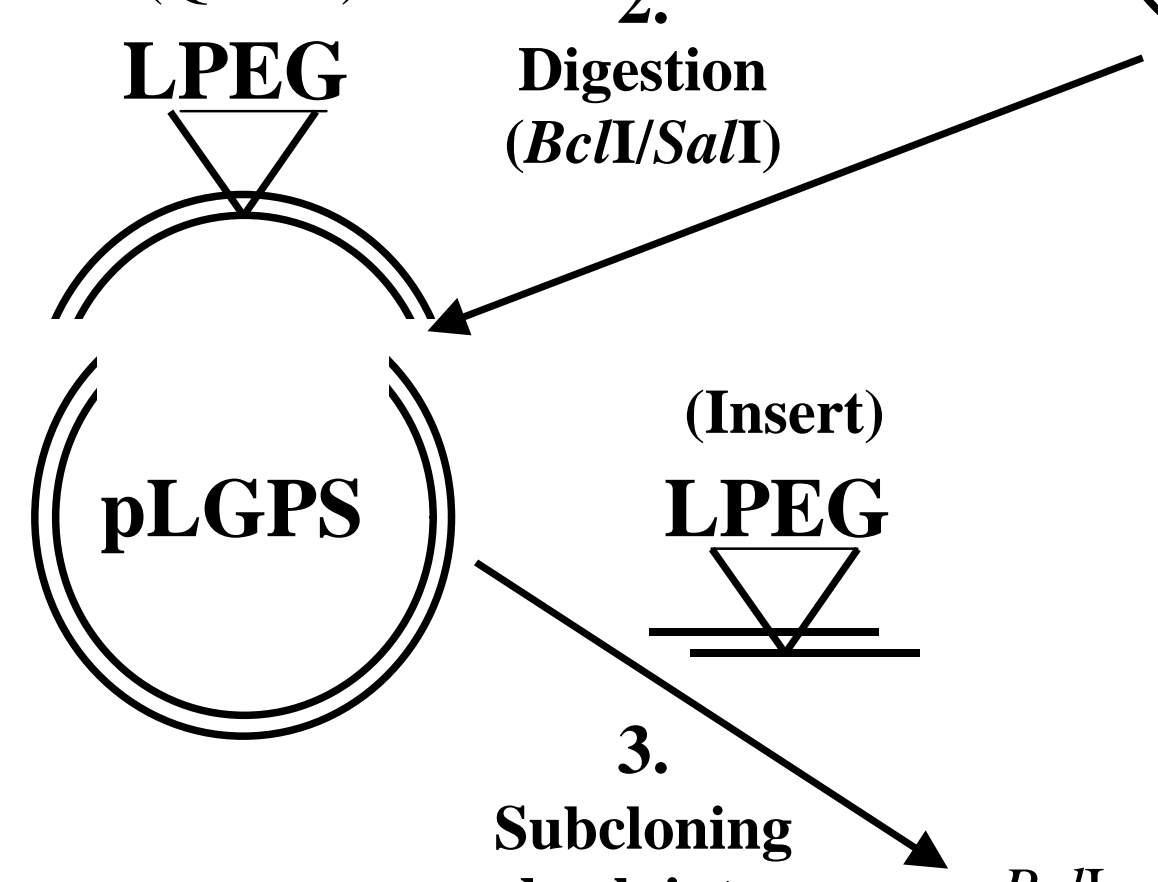
back into pLGPS

(Q190E)

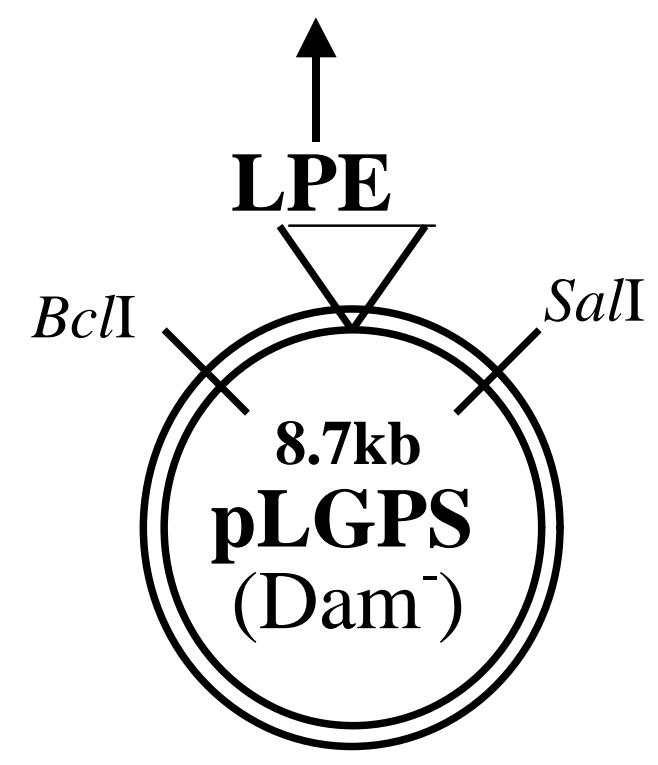


FIG. 4. Construction of the Q190H and $\mathrm{N}$ mutants of MLV RT. The Q190H and N mutants were generated through the utilization of the plasmid pESQ190E (template), the Q190H or Q190N single-stranded DNA oligonucleotides (mutagenic primers), and a Chameleon mutagenesis kit by Stratagene. The plasmid pESQ109E (pRMBNB derivative) is an MLV gag-pol expression construct and Dam $^{-}$denotes that the plasmid DNA was non-methylated. In addition, LPEG represents a mutated LPQG motif found in MLV RT containing the Q190E mutation in pol whereas the LPXG is a LPQG motif containing either the Q190H or Q190N mutation. To insure the absence of any undesired mutations introduced during mutagenesis, the $B c l$ I-SalI insert containing the appropriate mutation at position Q190 (Q190H or Q190N) was subcloned back into the backbone of pRMBNB, which was also digested with both enzymes. The Q190H and Q190N mutants were screened by digestion of plasmid with Bsu36I, since one of these sites had been destroyed during mutagenesis. The Q190H and Q190N mutations and the correct structure of the mutated plasmids were confirmed by the sequencing of the BclI-SalI fragments and through extensive restriction mapping. 


\section{A-4Construction of Q190H and N Mutants}
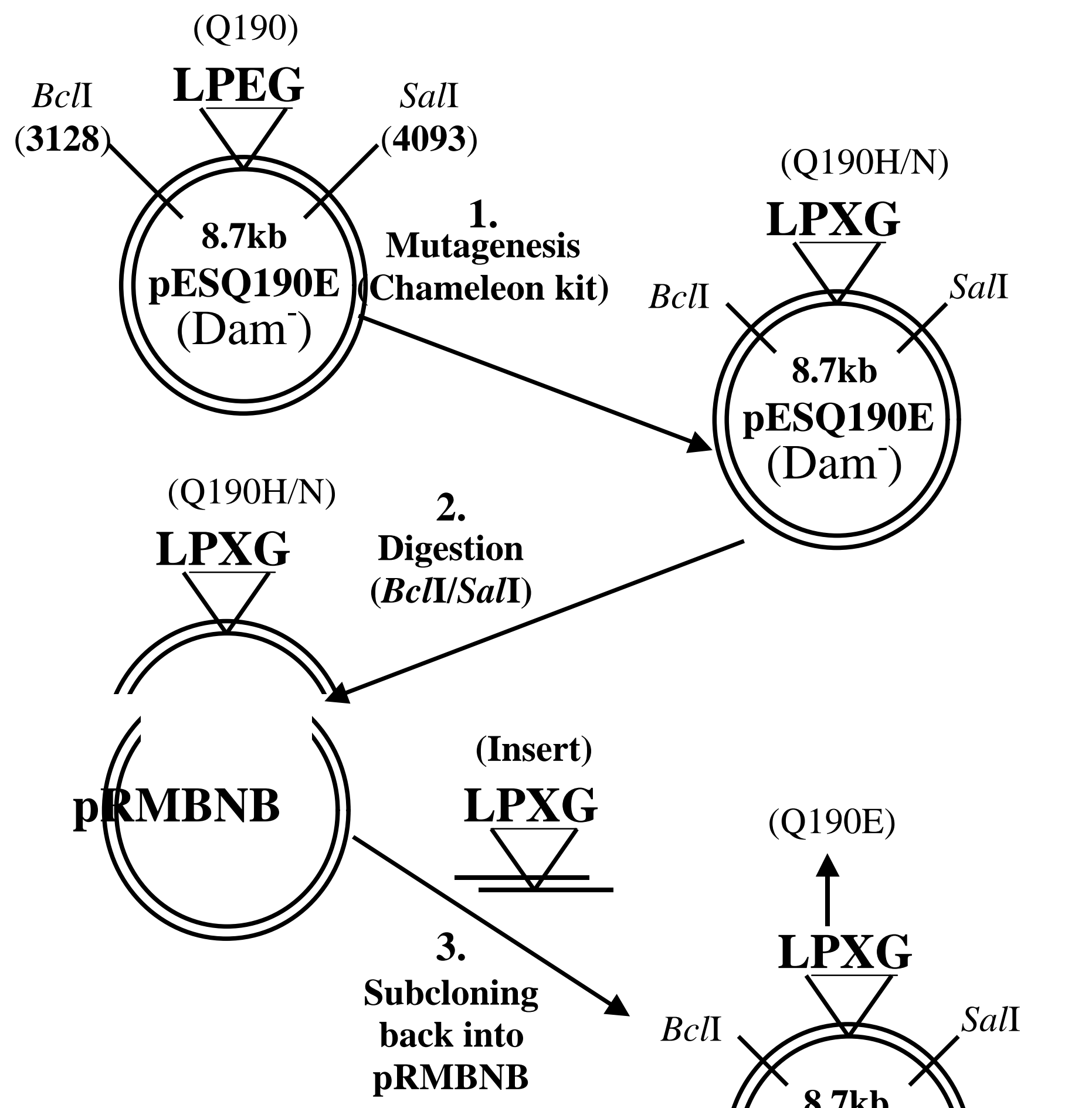

(Q190H/N)

LPXG
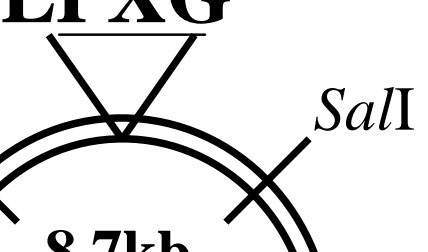

pESQ190E

(Dam)

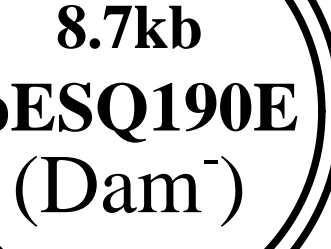

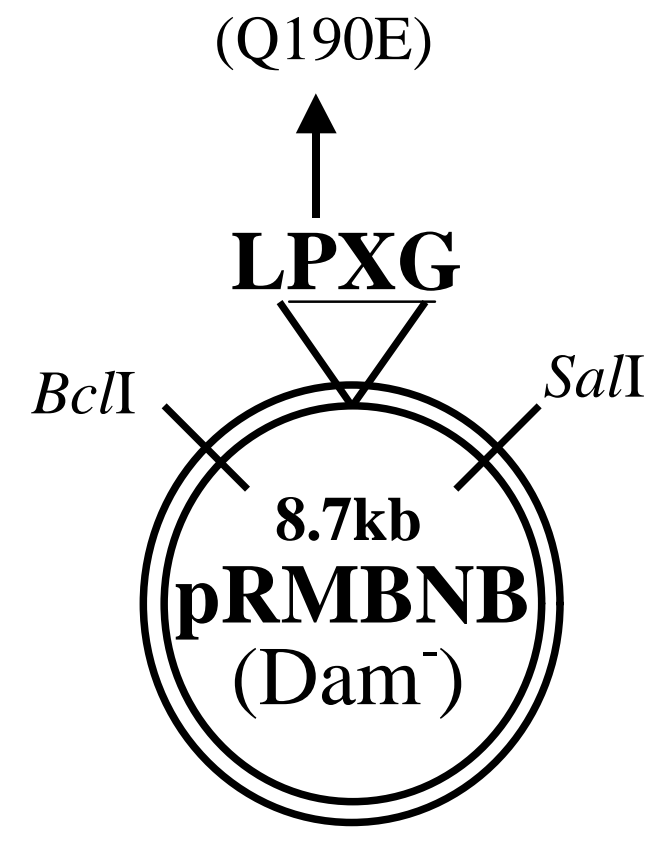


FIG. 5. Construction of the RMBNB derivative of pLGPS. The pRMBNB derivative of pLGPS was generated through the utilization of the plasmid pLGPS (template), two single-stranded DNA oligonucleotides (mutagenic primers), and a $5^{\prime}$ to $3^{\prime}$ mutagenesis kit. $\mathrm{pRMBNB}$ is a derivative of pLGPS with the addition of three unique restriction sites in pol (Bst1107I, NsiI, and BstBI) at nucleotide positions 3416, 3446, and 3496, which are locate in and around the dNTP-binding site of MLV RT. These unique restriction sites were introduced into pLGPS by two rounds of mutagenesis. During the first round, the $B s t 1107 \mathrm{I}$ and $B s t \mathrm{BI}$ sites were introduced and in the subsequent mutagenic reaction, the NsiI site was introduced. To insure the absence of any undesired mutations introduced during mutagenesis, the $B c l \mathrm{I}-B s p \mathrm{EI}$ insert containing the unique restriction sites (Bst1107I, NsiI, and BstBI) were subcloned back into the backbone of pLGPS, which was also digested with both enzymes. Dam ${ }^{-}$denotes that the plasmid DNA was non-methylated. The new generated pRMBNB was screened by digestion with Bst1107I, $N s i$, and BstBI. In addition, the unique restriction sites and the correct structure of the mutated plasmid was confirmed by the sequencing of the $B c l \mathrm{I}-B s p \mathrm{EI}$ fragment and through extensive restriction mapping. 


\section{A-5 Construction of RMBNB Derivative}
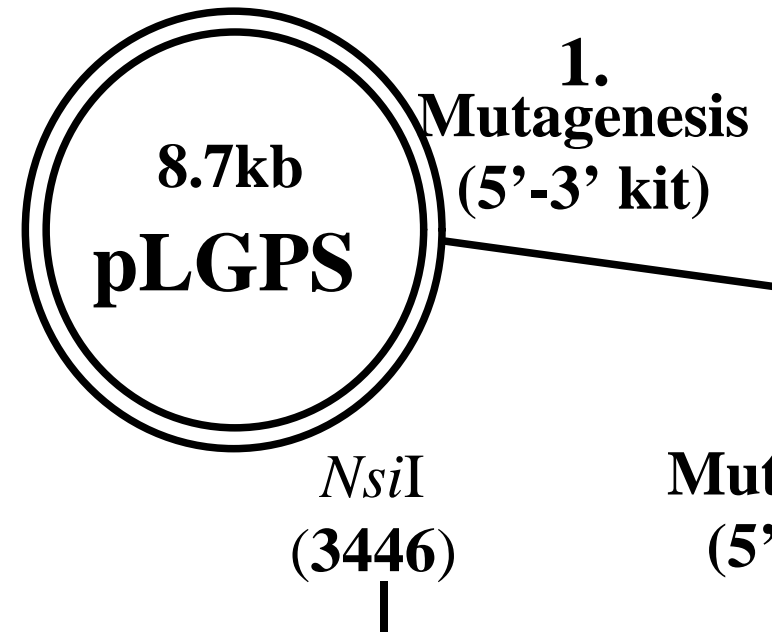

1.

Mutagenesis

(5'-3' kit)
Bst1107

(3416)

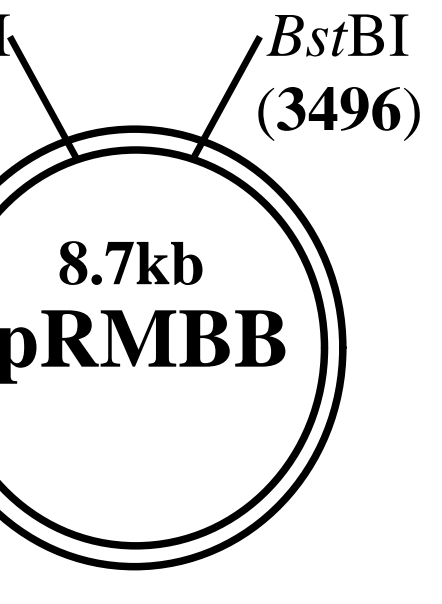

Bst11071

(3416)

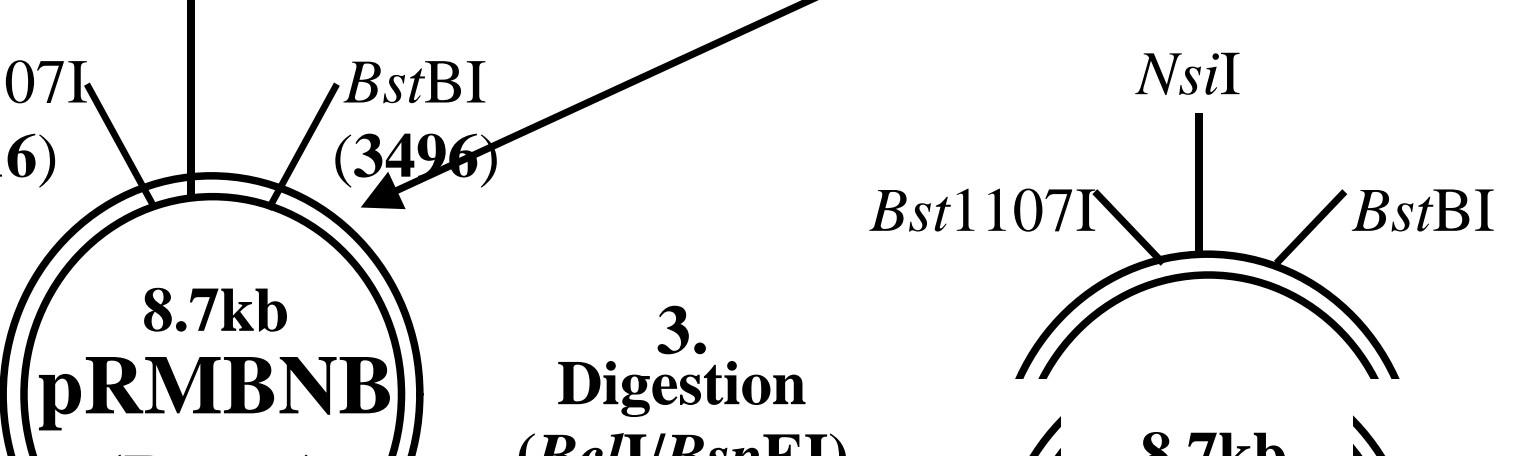

$\left(\mathrm{Dam}^{-}\right)-(\boldsymbol{B} \boldsymbol{c l} \mathbf{I} / \boldsymbol{B} \boldsymbol{s p} \mathbf{E I})$

(Insert)

2.

Mutagenesis

(5'-3' kit) 
FIG. 6. Construction of dNTP-binding site mutants of MLV RT utilizing synthesized double-stranded DNA oligonucleotides (dsDNA oligos) (Scheme I). The dNTP-binding site mutants of MLV RT generated by Scheme I involved replacing the Bst1107I-NsiI fragment of pRMBNB with a synthesized dsDNA oligo. These dsDNA oligos were complementary to the Bst1107I-NsiI fragment except for the appropriate mutation introduced in pol (specifically the dNTP-binding site of MLV RT). An additional mutation (silent) was present in these dsDNA oligos generating a new XbaI site utilized for screening. The plasmid pRMBNB was digested with Bst1107I and NsiI, the backbone was isolated, and the appropriate dsDNA oligos were cloned into these sites. Scheme I was employed to generate dNTP-binding site mutants of MLV RT for amino acid positions 147-153. 


\section{A-6 Construction of dNTP-Binding Site Mutants Utilizing dsDNA Oligos (Scheme I)}

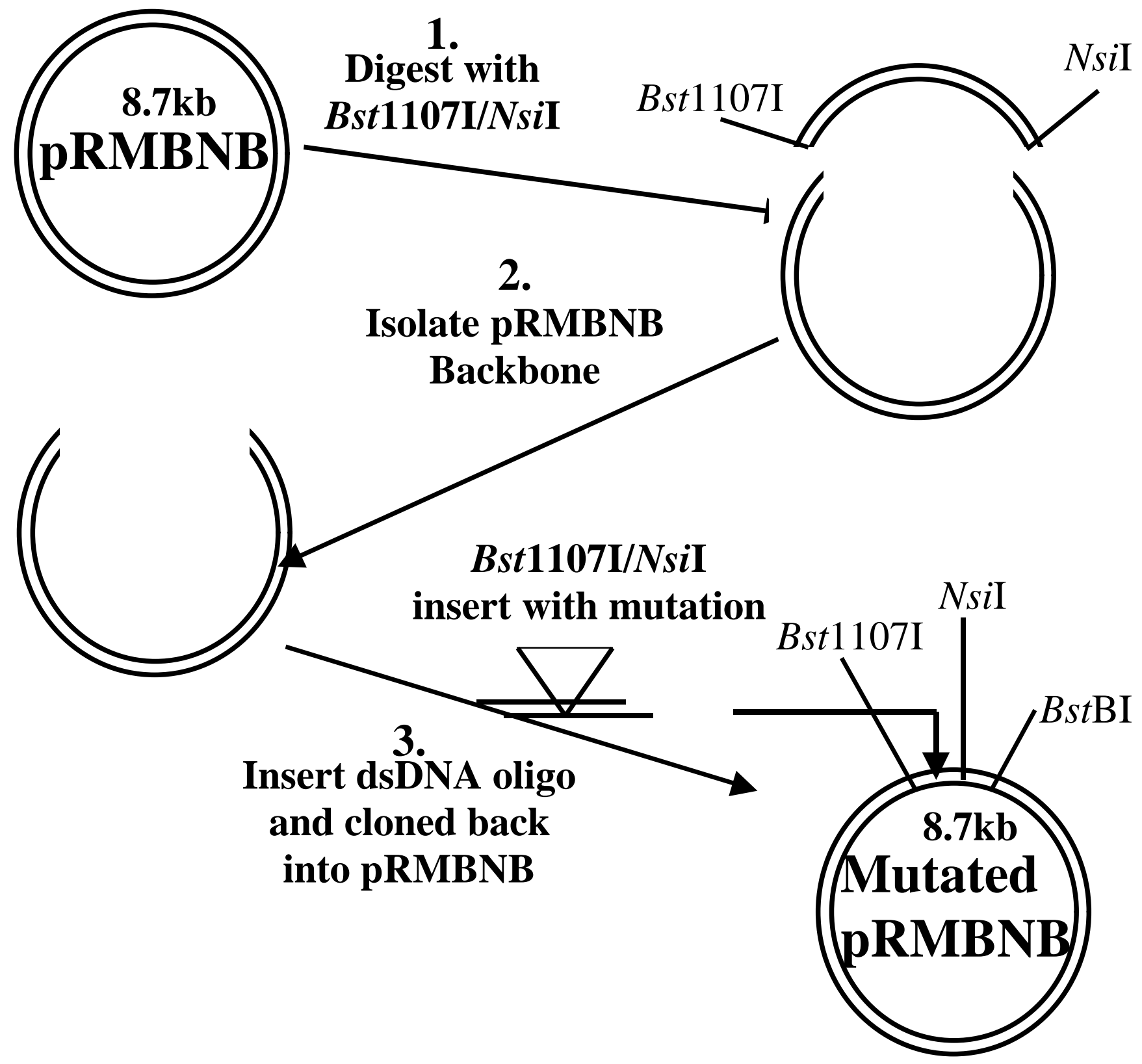


FIG. 7. Construction of dNTP-binding site mutants of MLV RT utilizing synthesized double-stranded DNA oligonucleotides (dsDNA oligos) (Scheme II). The dNTP-binding site mutants of MLV RT generated by Scheme II involved replacing the NsiI-BstBI fragment of pRMBNB with a synthesized dsDNA oligo. These dsDNA oligos were complementary to the $N s i \mathrm{I}-B s t \mathrm{BI}$ fragment except for the appropriate mutation introduced in pol (specifically the dNTP-binding site of MLV RT). An additional mutation (silent) was present in these dsDNA oligos generating a new SpeI site utilized for screening. The plasmid pRMBNB was digested with $N$ si I and BstBI, the backbone was isolated, and the appropriate dsDNA oligos were cloned into these sites. Scheme II was employed to generate dNTP-binding site mutants of MLV RT for amino acid positions 156-161. 
A-7 Construction of dNTP-Binding Site Mutants Utilizing dsDNA Oligos (Scheme II)

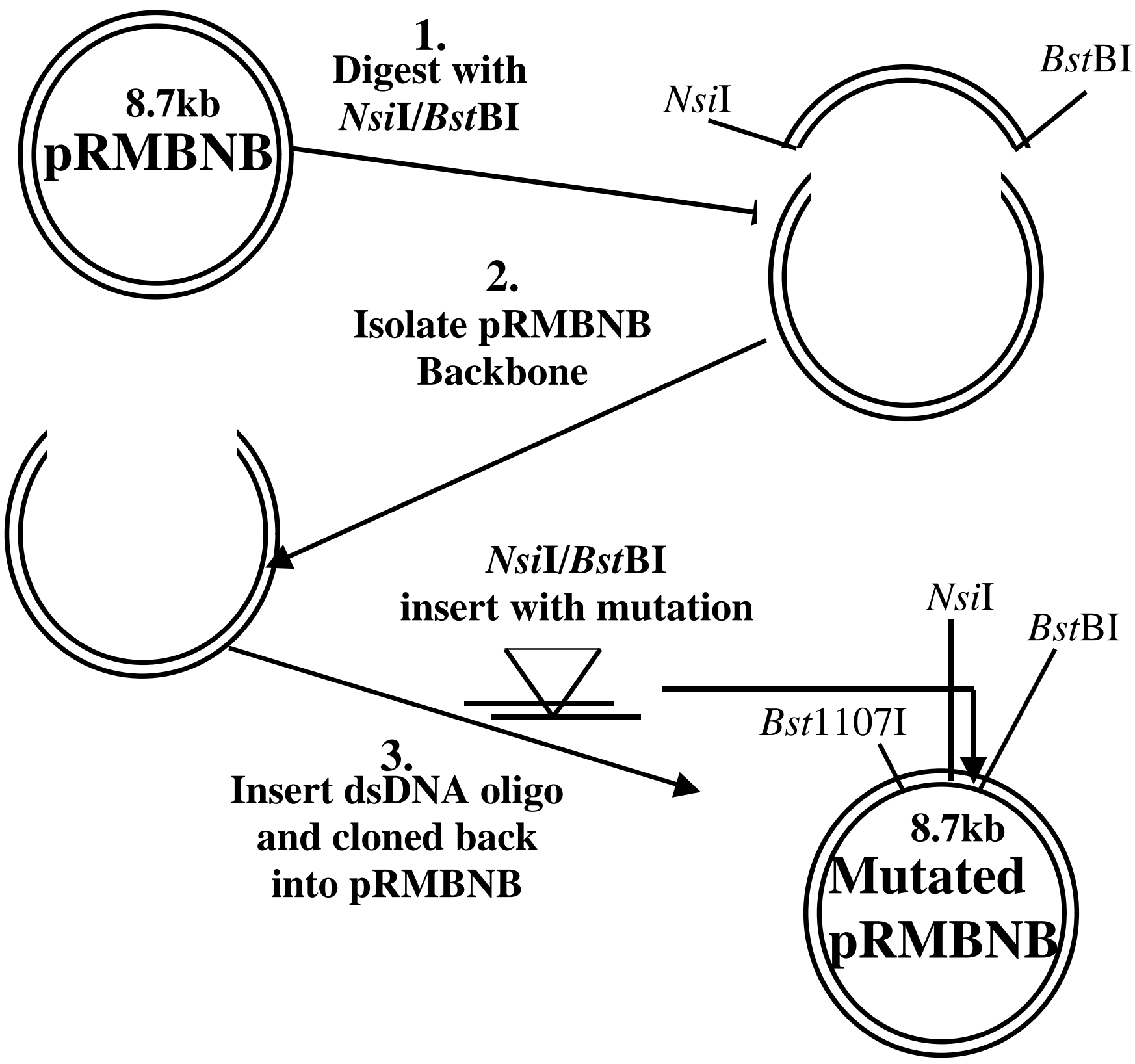


FIG. 8. Construction of dNTP-binding site mutants of MLV RT utilizing synthesized double-stranded DNA oligonucleotides (dsDNA oligos) (Scheme III). The dNTPbinding site mutants of MLV RT generated by Scheme III involved replacing the Bst $1107 \mathrm{I}-B s t \mathrm{BI}$ fragment of pRMBNB with a synthesized dsDNA oligo. These dsDNA oligos were complementary to the Bst1107I-BstBI fragment except for the appropriate mutation introduced in pol (specifically the dNTP-binding site of MLV RT). Additional mutations (silent) were present in these dsDNA oligos generating new XbaI and SpeI sites utilized for screening. The plasmid pRMBNB was digested with Bst1107I and $B s t \mathrm{BI}$, the backbone was isolated, and the appropriate dsDNA oligos were cloned into these sites. Scheme III was employed to generate dNTP-binding site mutants of MLV RT for amino acid positions 154-155. 
A-8 Construction of dNTP-Binding Site Mutants Utilizing dsDNA Oligos (Scheme III)
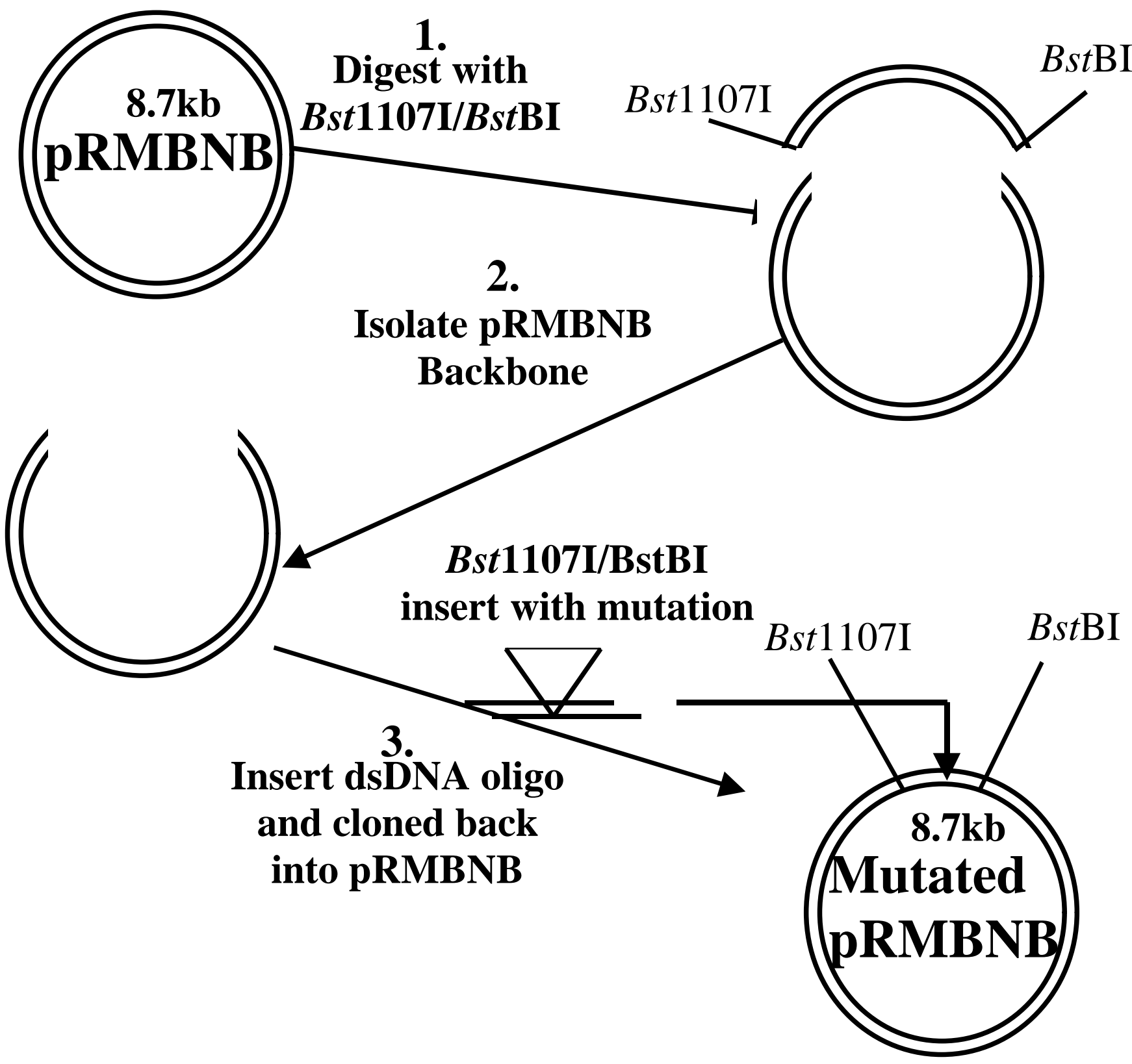
FIG. 9. Construction of dNTP-binding site mutants of MLV RT utilizing PCR-based mutagenesis. These mutants were generated individually through the utilization of either plasmid pLGPS or pRMBNB (template) and two sets of primers, each set containing a mutagenic primer with the appropriate mutation. Fragments containing the appropriate mutations were generated into two steps. During the first step, both a $5^{\prime}$ and $3^{\prime}$ fragment were generated, which overlapped at the codon being mutated ( $\mathrm{X}=$ mutation). Both fragments were isolated, purified, annealed to one another and extended in both directions. Product was isolated, purified, digested with appropriate restriction enzymes, and fragment was cloned back into either pLGPS or pRMBNB. Mutations and correct structure of mutated plasmids were confirmed by sequencing and extensive restriction mapping. This method was utilized in the generation of all K103 and R110 mutants as well as the Q190M mutant. Both the K103 and the R110 fragments for these mutants generated during the PCR-based mutagenesis were used to replace the BclI-Bst1107I fragment of pLGPS or pRMBNB. The Q190M fragment for this mutant generated during the PCR-based mutagenesis was used to replace the NsiI-SalI fragment of pLGPS or pRMBNB. 
A-9 Construction of dNTP-Binding Site Mutants by PCR-Based Mutagenesis
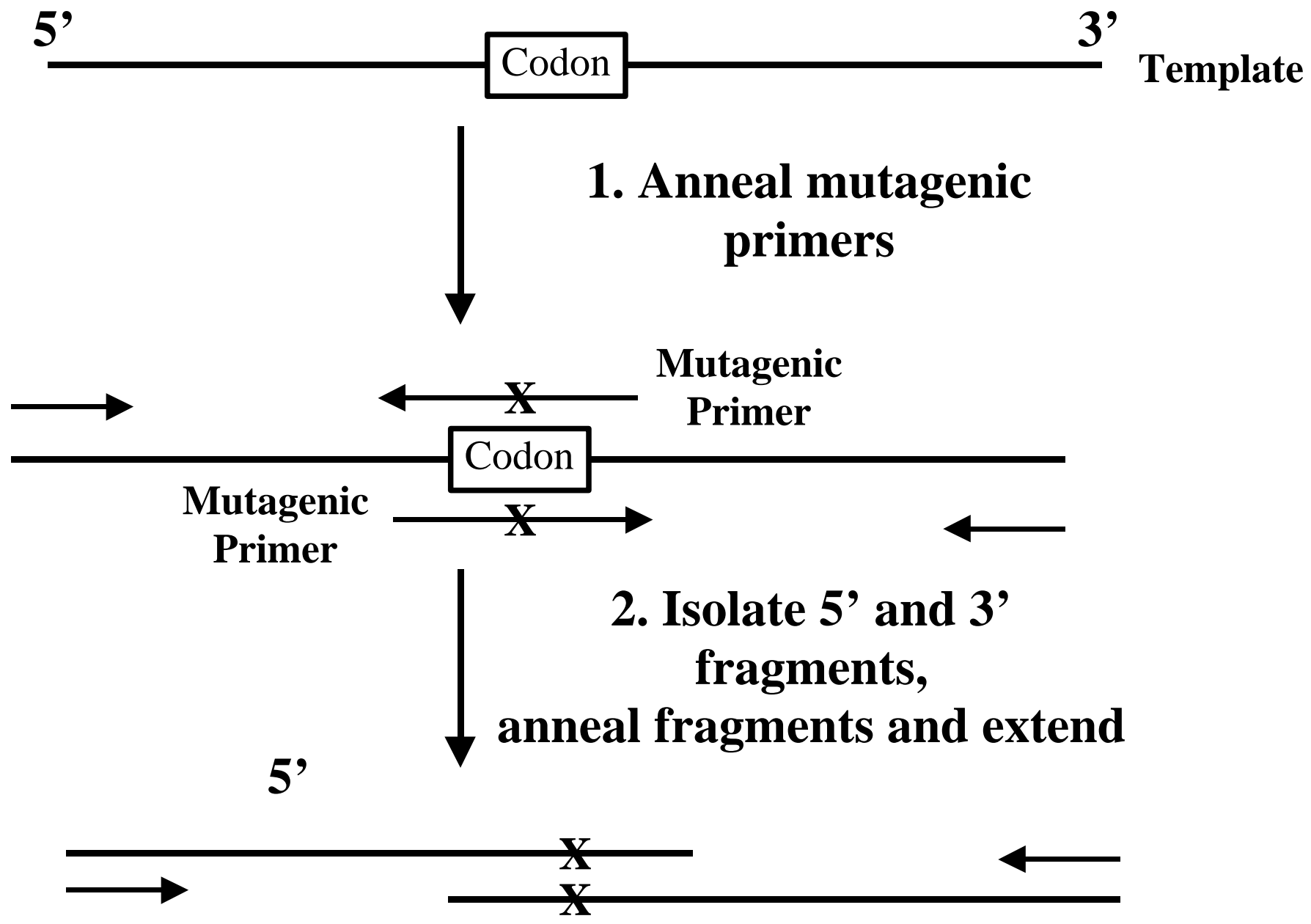

3'

3. Isolate dsDNA fragment 


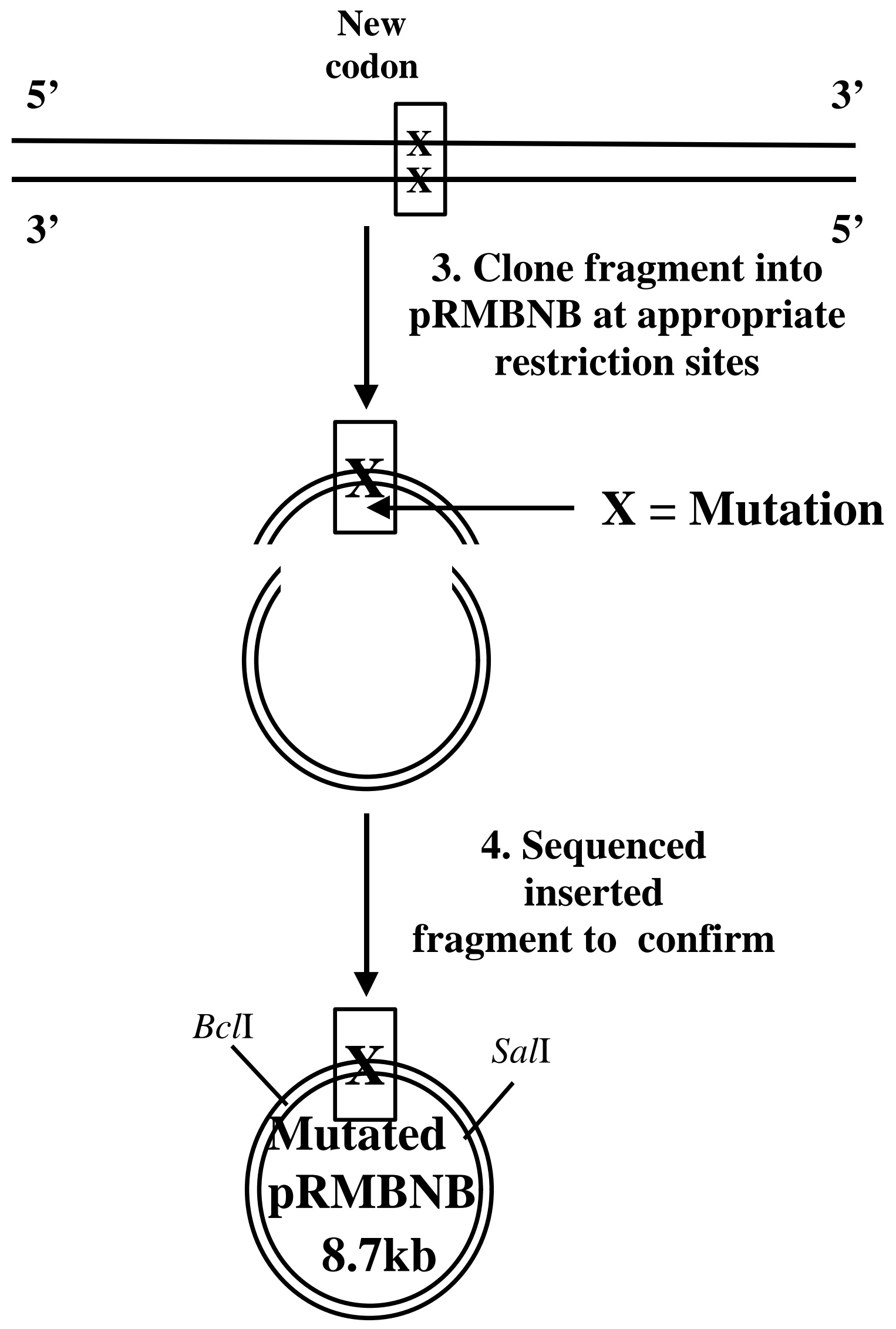




\section{ABSTRACTS}




\section{STRUCTURAL DETERMINANTS OF MURINE LEUKEMIA VIRUS (MLV) REVERSE TRANSCRIPTASE (RT) IMPORTANT FOR FIDELITY.}

Elias K. Halvas, Jenny S. Svarovskaia, Ronald A. Mudry, Jr., and Vinay K. Pathak, Department of Biochemistry and Mary Babb Randolph Cancer Center, West Virginia University, Morgantown, WV 26505 Retroviruses exhibit high mutation rates that may be attributed to RT. Structural features of RTs that are important for accuracy of DNA synthesis are currently unknown. Potential structural determinants of RT that may be important for fidelity include the YXDD box, the conserved LPQG sequence, the dNTP binding site, the thumb domain, and the RNase $\mathrm{H}$ domain.

We have developed a rapid in vivo assay to identify structural determinants associated with fidelity of MLV RT. The assay employs a D17-based encapsidating cell line (ANGIE P) that expresses the MLV envelope gene and an MLV-based retroviral vector. The MLV-based vector encodes the $\beta$-galactosidase gene (lacZ) which serves as a reporter of mutations and the neomycin drug resistance gene. The MLV gagpol construct expressing the RT can be mutated at sites thought to be important for fidelity; the ANGIE P cells are transfected with the wildtype or mutated MLV gag-pol constructs, and virus produced is used to infect D17 target cells. Drug resistant colonies formed after one cycle of replication are X-Gal stained and the rate of inactivation of lacZ is determined. The relative change in fidelity can be assessed by comparing mutant RTs with the wildtype RT.

We have mutated the second amino acid in the highly conserved YVDD box to generate V223M and V223S mutants of MLV RT. In addition, RNase H domain mutants S526A and R657S were created. Utilization of wildtype RT in our assay resulted in inactivation of $l a c Z$ at a frequency of $5.2 \%$ per cycle. In comparison, the V223M and the V223S mutants inactivated lacZ at higher rates of $9.2 \%$ and $12.3 \%$ per cycle, respectively. The S526A and R657S RNase H mutants also inactivated lacZ at higher rates of $8.5 \%$ and $7.2 \%$ per cycle, respectively. These results demonstrate that we have developed a system to identify structural determinants important for fidelity. In addition, the second amino acid of the YVDD box and the structure of the RNase $\mathrm{H}$ domain play a role in the accuracy of DNA synthesis. 


\section{STRUCTURAL DETERMINANTS OF THE PUTATIVE dNTP-BINDING SITE IN MURINE LEUKEMEIA VIRUS (MLV) REVERSE TRANSCRIPTASE IMPORTANT FOR FIDELITY.}

Elias K. Halvas, Jenny S. Svarovskaia, Erin White, Ronald A. Mudry, Jr., and Vinay K. Pathak, Department of Biochemistry and Mary Babb Randolph Cancer Center, West Virginia University, Morgantown, WV 26505

Retroviruses exhibit high mutation rates, primarily because retroviral RTs carry out error-prone DNA synthesis. In vitro studies have indicated that several structural determinants of RT are important for fidelity, which include the YXDD box, Q190 of HIV-1 RT, the dNTP-binding site, the thumb domain, and the RNase H domain. However, the structural features of RTs that are important for the accuracy of DNA synthesis during retroviral replication are currently unknown.

We have developed a rapid in vivo assay to identify structural determinants associated with fidelity of MLV RT. The assay employs a D17-based encapsidating cell line (ANGIE P) containing an MLV-based vector encoding the lacZ gene, which serves as a reporter of mutations. The MLV gag-pol construct expressing RT was mutated at sites thought to be important for fidelity; the ANGIE P cells were transfected with the wild-type or the mutated gag-pol constructs, and the virus produced was used to infect D17 target cells. Drug-resistant colonies formed after one cycle of replication were stained with X-Gal and the rate of $l a c Z$ inactivation was determined. The relative change in fidelity was assessed by comparing mutant RTs to the wild-type RT, which inactivated the lacZ gene at a frequency of $5.5 \% \pm 0.3 \%$.

To determine the importance of the putative dNTP-binding site to the accuracy of DNA synthesis, amino acid positions 147-161 of the MLV RT were subjected to extensive mutational analysis. Three of the amino acids in this region of MLV RT (D153, A154, and F155) are homologous to amino acids D113, A114, and Y115 of HIV-1 RT, which have been shown to contact the substrate dNTP. In addition, mutations were introduced at position Q190 (equivalent to Q151 of HIV-1 RT).

A total of 28 different mutants were generated and tested. Fourteen of the mutants exhibited virus titers that were at least 10,000-fold lower than the titer of the wild-type MLV, and their fidelity could not be determined. Four mutants did not exhibit a change in fidelity. The remaining mutants generated mutant frequencies that were 1.2- to 2.6-fold higher than the wild type. The D153A mutant exhibited a very low virus titer and a mutant frequency of $8.2 \%$. The F155 was mutated to D, E, I, M, V, W, and Y. The mutants F155D, F155E, F155I, F155M, and the F155V had undetectable viral titers and their effect on fidelity could not be determined. The F155W exhibited a very low viral titer and a mutant frequency of $14.8 \%$. The F155Y mutant exhibited a viral titer that was $31 \%$ to that of the wild type; the mutant did not display a change in fidelity. All three mutants with substitutions at the Q190 position had undetectable viral titers.

The results indicate that the F155 position of MLV RT is highly conserved since most of the substitutions severely reduced the viral titers. These results also demonstrate that several amino acids of the putative dNTP-binding site of MLV RT can affect the fidelity of DNA synthesis during retroviral replication. 


\section{CURRICULUM VITAE}




\section{OBJECTIVE}

To pursue a career as a Research Scientist using my experience in biochemistry, molecular biology, cellular biology, and immunology to develop diagnostics and treatments for human diseases.

\section{EDUCATION}

Ph.D. in Biochemistry

Department of Biochemistry, School of Medicine,

Mary Babb Randolph Cancer Center,

West Virginia University, Morgantown, WV

Dissertation: Identifying Structural Determinants of Murine Leukemia Virus

Reverse Transcriptase Important for Fidelity and Drug-Resistance In Vivo.

B.A. in Microbiology

1992

Concentration in molecular biology, School of Arts and Sciences,

University of Pittsburgh, Pittsburgh, PA

\section{RESEARCH EXPERIENCE}

Graduate Research in the laboratory of Dr. Vinay K. Pathak,

1994-present

Mary Babb Randolph Cancer Center,

Department of Biochemistry, School of Medicine,

West Virginia University, Morgantown, WV

Research Technician in the laboratory of Dr. Nirmala SunderRaj,

1992-1994

Department of Ophthalmology, School of Medicine,

University of Pittsburgh, Pittsburgh, PA

Research Internship in the laboratory of Dr. Christine Milcarek, Department of Biochemistry, School of Medicine,

University of Pittsburgh, Pittsburgh, PA

\section{Graduate Research Summary}

(i) I have examined fidelity of murine leukemia virus (MLV) reverse transcriptase (RT) during in vivo DNA synthesis and have identified structural determinants with in the polymerase that may be crucial in this mechanism. This was accomplished through the development of a rapid in vivo assay. Utilizing this rapid in vivo assay, I have examined the role that the V223 position of the MLV RT YVDD motif plays in fidelity of DNA synthesis, viral replication, and RT activity. I have observed through the utilization of the rapid in vivo assay that substitutions at the V223 position of MLV RT are correlated with changes in fidelity and reductions in both virus titers as well as polymerase activity. These findings suggest that the V223 position of MLV RT may exert effects that are important for fidelity of in vivo DNA synthesis during reverse transcription.

(ii) I have also examined the role of several amino acid positions constituting the putative dNTP binding site of MLV RT and the effects these amino acids exert on fidelity, virus titers, and RT activity. I have observed through extensive site-directed mutagenesis and the utilization of the rapid assay that several positions in this pocket of MLV RT alter fidelity and severely affect viral replication as well as polymerase activity. These findings suggest a critical role for several of these residues in accurate in vivo DNA synthesis during reverse transcription, viral replication, and RT activity.

(iii) I have examined the mechanism of 3TC drug-resistance in vivo associated with V223 position of MLV RT. As expected, I as well as others, have observed that the wild type MLV RT containing the YVDD motif was resistant to 3TC. In contrast to 3TC-resistance in HIV-1 RT, various mutants of the V223 
position (V223M, V223I, V223A, and V223S) were also resistant to 3TC. These findings suggest that although MLV and HIV-1 RTs are similar, structural differences between the two may account for different drug-sensitivity profiles.

\section{Technician Research Summary}

(i) I have examined diversities in the extracellular matrices (ECMs) associated with the cornea as well as the surrounding limbial epithelium and stroma. I have identified that the long variant of type XII collagen is a component of both the corneal and limbal ECMs. These findings suggest the long variant of type XII collagen may contribute to differences in the basement membrane zones of the cornea and limbus.

\section{TECHNICAL SKILLS}

\section{Biochemistry}

- Harvesting antibodies from hybridoma

- Immuno-precipitation of antibodies and purification by affinity chromatography

- Western blot analysis and ELISA

- Chemiluminescence detection

- Exogenous reverse transcriptase assays

- Slot blot analysis

\section{Molecular Biology}

- DNA cloning

- DNA sequencing (manual and automated)

- PCR

- Double stranded DNA oligo-based mutagenesis

- PCR-based mutagenesis

- DNA and RNA extraction and purification

- Extraction of viral RNA

- Southern blot analysis

\section{PROFESSIONAL AND LEADERSHIP SKILLS}

- Responsible for the direction of summer assistants assigned to me.

- Manuscript writing for publication

- Conducted seminar presentations (Cold Spring Harbor Laboratory retrovirus meeting and Van Leere meeting)

- Poster presentation (Cold Spring Harbor Laboratory retrovirus meeting)

- Various computer skills relating to my field (DNASIS and Ras Mol)

- Teaching experience (Problem based learning for medical and dental students)

- Instructed a medical technology laboratory for undergraduates

\section{Cell Culture}

Maintenance of several cell lines

- DNA stable transfections

- Isolation of cell clones

- Infection of several cell types

- Concentration of virus
- Lowery and Bio Rad protein assays

- FPLC

- X-Gal staining of mammalian cells

- Cell extract preparation (Amplification of DNA and detection of protein)

- Conformational Polymorphism Gel Electrophoresis 


\section{PUBLICATIONS}

Wessel, H., S. Anderson, D. Fite, E. Halvas, j. Hempel, and N. SundarRaj. 1997. Type XII collagen contributes to diversities in human corneal and limbal extracellular matrices. Invest. Ophthalmol. Vis. Sci. 38:2408-2422.

Halvas, E., K., E. S. Svarovskaia, and V. K. Pathak. 2000. Development of an in vivo assay to identify structural determinants in murine leukemia virus reverse transcriptase important for fidelity. J. of Virol. 74:312-319.

Halvas, E., K., E. S. Svarovskaia, E. O. Freed, and V. K. Pathak. Wildtype and YMDD Mutant of Murine Leukemia Virus Reverse Transcriptase are Resistant to 2', 3'-dideoxy-3'-thiacytidine (3TC). Manuscript submitted.

Halvas, E., K., E. S. Svarovskaia, and V. K. Pathak. Structural determinants in the putative dNTP binding site of MLV RT that are important for in vivo fidelity during reverse transcription. Manuscript in preparation.

\section{ABSTRACTS}

Halvas, E., K., E. S. Svarovskaia, and V. K. Pathak. 1997. Identifying structural determinants in murine leukemia virus reverse transcriptase important for fidelity in vivo. Oral presentation, Cold Spring Harbor Laboratory, Cold Spring Harbor, N. Y.

Halvas, E., K., E. S. Svarovskaia, E. White, R. A. Mudry, JR., and V. K. Pathak. 1999. Structural determinants in the putative dNTP binding site of MLV RT that are important for in vivo fidelity during reverse transcription. Poster, Cold Spring Harbor Laboratory, Cold Spring Harbor, N. Y. 


\section{References}

Dr. Vinay K. Pathak

HIV Drug Resistance Program

DBS NCI FCRDC, Bldg. 535, Rm. 334

Frederick, MD 21702

Phone: (301) 846-1710

Fax: (301) 846-6013

Electronic mail address: vpathak@mail.ncifcrf.gov

Dr. Mike Miller

Department of Biochemistry, School of Medicine

West Virginia University

Morgantown, WV 26506

Phone: (304)-293-7762

Fax: (304)-293-

Electronic mail address: mmiller@hsc.wvu.edu

Dr. Nirmala SundarRaj

Department of Ophthalmology, School of Medicine

University of Pittsburgh

Pittsburgh, PA 15261

Phone: (412)-647-2236

Electronic mail address: sundarragn @ msx.upmc.edu

Dr. Christine Milcarek

Department of Biochemistry, School of Medicine

University of Pittsburgh

Pittsburgh, PA 15261

Phone: (412)-648-9098

Fax: (412)-624-1401

Electronic mail address: milcarek@ pitt.edu 